\title{
MÉTODOS DE COMPARAÇÃO 1)E SÉRIES TEMPORAIS
}

\author{
Gladys Elena Salcedo Echevery
}

\author{
DISSERTAÇÃO APRESENTADA \\ $\mathrm{AO}$ \\ INSTITUTO DE MATEMÁTICA E ESTATÍSTICA \\ DA \\ UNIVERSIDADE DE SÃO PAULO \\ PARA \\ OBTENÇÃO DO GRAU DE MESTRE \\ EM \\ ESTATÍSTICA \\ Área de Concentração: Séries Temporais \\ Orientador: Profa. Dra. Clélia Ma. de Castro Toloi
}

Este trabalho foi financiado pela "Universidad del Quindio-Colombia" e pelo CNPq-Brasil

-São Paulo, Março de 1999- 


\title{
MÉTODOS DE COMPARAÇÃO DE SÉRIES TEMPORAIS
}

\author{
Este exemplar corresponde à redação \\ final da dissertação devidamente corrigida \\ e defendida por Gladys Elena Salcedo Echeverry \\ e aprovada pela comissão julgadora.
}

São Paulo, 31 de Março de 1999.

Banca examinadora:

- Profa. Dra. Clélia Ma. de Castro Toloi (orientadora) - IME-USP

- Prof. Dr. Pedro Alberto Morettin - IME-USP

- Profa. Dra. Silvia Regina da Costa Lopez - UFRGS 
Ao meu pãe, meus irmãos

e especialmente a memória de minha mãe. 


\section{Agradecimentos}

À Universidad del Quindío, por ter me liberado o tempo necessário para a realização do mestrado.

À profesora Clélia Maria de Castro Toloi, por sua excelente orientação tanto acadêmica quanto humana e pela dedicação para concluir esta dissertação.

Aos meus professores do IME-USP, pelo conhecimento transmitido.

Aos professores Pedro Alberto Morettin e David Ross Brillinger, pelas sugestões apresentadas.

À minha família, pelo seu carinho e constante apoio logístico, especialmente às minhas irmãs Socorro e Marleny, que embora distantes, sempre estiveram ao meu lado.

Ao meu colega Luis Hernando Hurtado, com quem pensei no problema desta dissertação.

À CNPq e a Universidad del Quindío, pelo apoio financeiro.

Finalmente, agradeço especialmente a todos os meus colegas e amigos colombianos, brasileiros e outros estrangeiros, que tornaram mais tranqüilos alguns momentos difíceis e mais agradável minha estadia no Brasil. 


\section{Resumo}

$\mathrm{Na}$ análise de séries temporais, muitas vezes, é de interesse verificar se duas séries, ou trechos de uma mesma série, estão sendo gerados pelo mesmo processo estocástico. No caso de processos estacionários de segunda ordem, as hipóteses de interesse são verificar se elas apresentam igual estrutura de autocovariância (autocorrelação) ou igualdade das funções de densidades espectrais.

Nesta dissertação apresentamos vários testes, alguns para séries multivariadas, com esse objetivo. Simulações são realizadas para comparar os diferentes procedimentos no caso de comparação de duas séries univariadas.

Finalmente, aplicamos todas as técnicas para a análise de um conjunto de dados reais. 


\section{Abstract}

In time series analyses sometime is interesting to verify if two series or two parts of the same series are realizations of the same stationary process. Considering the class of the second order stationary process, statistical test procedures are presented to compare the autocovariance, the autocorrelation and the spectral functions of univariate and multivariate time series. Simulations were used to compare the powers of the various univariate tests. An application with real series is given. 


\section{Conteúdo}

$\begin{array}{lll}\text { Capítulo } 1 & \text { Introdução } & 1\end{array}$

Capítulo 2 Conceitos Básicos e Definições Preliminares 5

Capítulo 3 Análise Espectral de Processos Estacionários 14

3.1 Processos Univariados . . . . . . . . . . . . . . . . . 14

3.2 Processos Multivariados . . . . . . . . . . . . . . . 17

Capítulo 4 Análise Temporal de Processos Estacionários 23

4.1 Processos Univariados . . . . . . . . . . . . . . . . . . 23

4.1 .1 Processos Lineares . . . . . . . . . . . . . . . . 26

4.1.2 Processos Auto-regressivos de Médias Móveis . . . . . . . 28

4.1.3 Processos não Estacionários e Processos ARIMA . . . . . 31

4.1.4 Processos Sazonais e Modelos SARIMA . . . . . . . . 33

4.2 Processos Multivariados . . . . . . . . . . . . . 35

4.2 .1 Processos Lineares Vetoriais Gerais . . . . . . . . . . . 35

4.2 .2 Processos ARMA Vetoriais . . . . . . . . . . . 36

4.2.3 Processos ARMA Vetoriais não Estacionários . . . . . . . 37

Capítulo 5 Métodos de Comparação de Séries Temporais $\quad 38$

5.1 Comparação de Duas Séries Univariadas . . . . . . . . . . . 38

5.1.1 Teste das Somas Acumuladas (SA-Coates e Diggle) . . . . 39

5.1.2 Teste da Razão de Verossimilhança (RV-Coates e Diggle) 41

5.1.3 Teste de Igualdade das Funções de Autocovariância (Mélard e Roy $\ldots \ldots \ldots \ldots \ldots \ldots$ 
5.1.4 Teste de Igualdade das Funções de Autocorrelação (Quenouille) . . . . . . . . . . . . . . . 4 47

5.2 Comparação de Duas Séries Multivariadas . . . . . . . . . . . . . 48

5.2.1 Comparação de Séries no Caso de Independência (Carmona e Wang) . . . . . . . . . . . . . . . 50

5.2.2 Comparação de Séries no Caso de Dependência (Carmona e Wang) . . . . . . . . . . . . . . . 52

$\begin{array}{lll}\text { Capítulo } 6 & \text { Simulações } & 56\end{array}$

6.1 Testes Univariados . . . . . . . . . . . . . . . . . . 56

6.2 Testes Multivariados . . . . . . . . . . . . . . . 60

Capítulo 7 Aplicações $\quad 66$

7.1 Análise Univariada das Séries . . . . . . . . . . . . . . . 66

7.1.1 Análise de Salinidade . . . . . . . . . . . . 66

7.1 .2 Análise de Temperatura . . . . . . . . . . . . . 78

7.2 Análise Multivariada das Séries . . . . . . . . . . . . 88

7.2.1 Testes de Carmona e Wang . . . . . . . . . . . . . . 89

7.3 Conclusões . . . . . . . . . . . . . . . . . . . . . 92

Referências $\quad 94$

Apêndice A Séries de Salinidade e Temperatura $\quad 97$

Apêndice B Resultados das Simulações para Séries Univariadas 103

$\begin{array}{lll}\text { Apêndice C } & \text { Teste de Kolmogorov-Smirnov } & 106\end{array}$

$\begin{array}{lll}\text { Apêndice D Programas no S-Plus } & 108\end{array}$ 


\section{Capítulo 1}

\section{Introdução}

O assunto desta dissertação foi motivado por um estudo feito para algumas variáveis físicas, químicas e biológicas, medidas no principal eco-sistema lagoaestuarino colombiano que tem por nome "La Ciénaga Grande de Santa Marta (CGSM)". Pelas suas características geomorfológicas (forma, profundidade, entradas de água doce, comunicações com o mar) a CGSM é um sistema lagoaestuarino. Suas principais fontes de água doce são constituídas pelo rio Magdalena (maior rio colombiano) ao oeste e vários rios provenientes da "Sierra Nevada de Santa Marta (SNSM)" ao leste. Localizada sobre a costa caribenha da Colômbia, no "Departamento del Magdalena", a CGSM é a maior lagoa costeira do país e, tradicionalmente, tem sido uma das principais fontes de fornecimento de peixes e mariscos para a costa norte colombiana e ainda para mercados do interior do país.

A CGSM junto com a ilha de Salamanca e o conjunto de lagoas e canais adjacentes, denominados "Complejo de Pajarales y Santuario de Fauna y Flora", compreende uma área total de 1280 quilômetros quadrados, sendo que a CGSM compreende uma área de 450 quilômetros quadrados. Todo esse complexo faz parte do Delta Exterior do Rio Magdalena (DERM), ver Figura 1.1.

Desde 1956, aproximadamente, este eco-sistema tem sofrido um notório deterioramento refletido na hipersalinização dos seus solos e, conseqüentemente, na morte massiva do bosque de mangue, diminuição substancial na diversidade e abundância de peixes, aves e moluscos, contaminação das suas águas por sedimentos e organismos com pesticidas organoclorados, metais pesados e bactérias pa- 


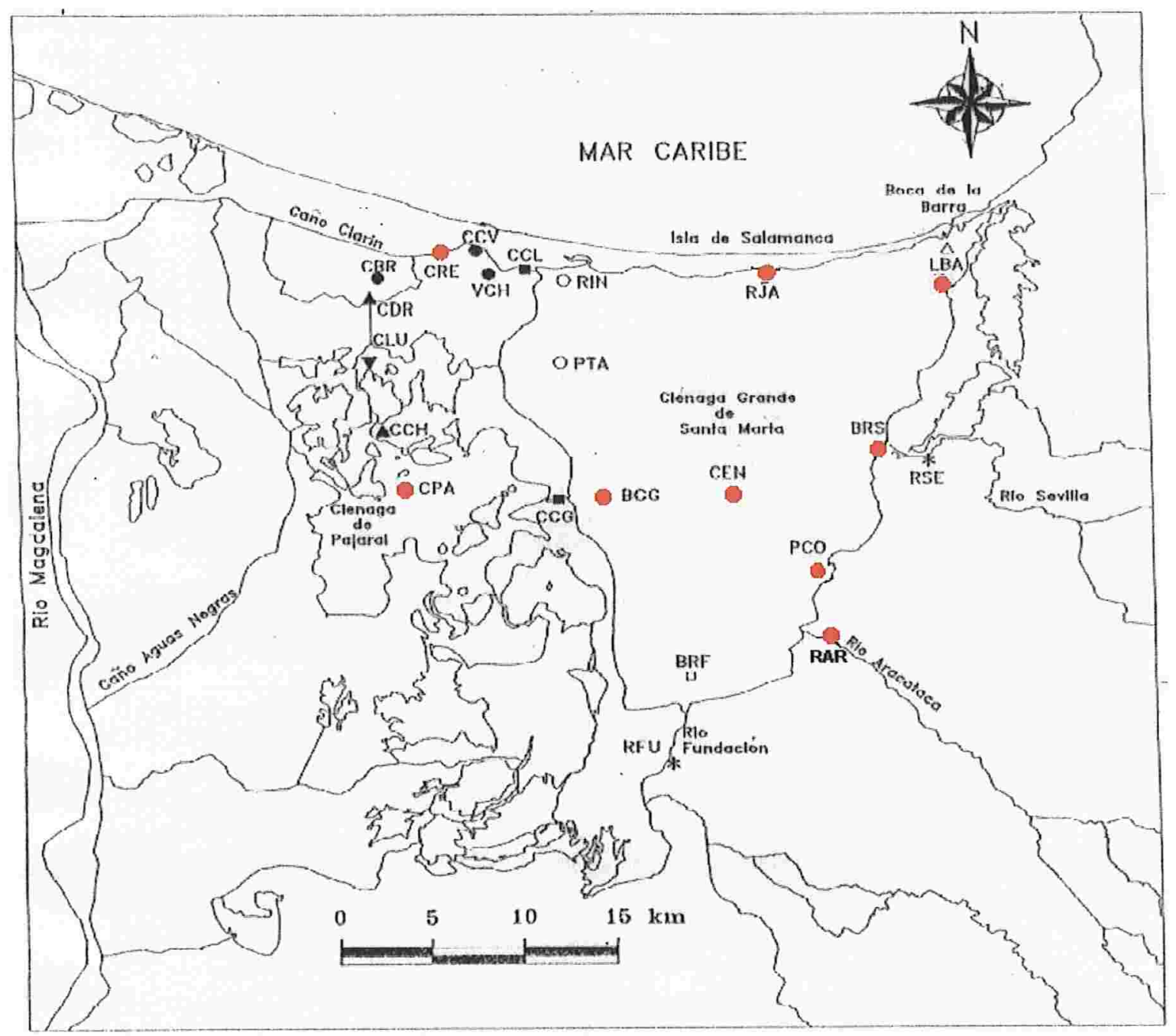

Figura 1.1. Delta Exterior del Rio Magdalena. 
togênicas. Também se tem comprovado um progressivo e significativo incremento na salinidade dos corpos de água e concentração do material em suspensão. O impacto de origem antrópico exercidos sobre a CGSM nas últimas quatro décadas, foram classificadas como: alterações físicas, enriquecimento com matéria orgânica e/ou nutrientes orgânicos, introdução de sustâncias tóxicas e alteração direta da estrutura das comunidades de fauna e flora. Para maiores detalhes, consultar Botero e Mancera (1996).

Pelos diferentes estudos feitos neste eco-sistema, dispõe-se de várias medições mensais de várias variáveis físicas, químicas e biológicas, entre elas, temperatura, salinidade, oxigênio, $\mathrm{pH}$, nitritos, nitratos, ortofosfatos e outras, sendo que estas medições tem sido tomadas em diferentes estações do eco-sistema. Por outro lado, variáveis como salinidade, temperatura e oxigênio têm sido medidas em três profundidades diferentes, a saber, superfície, fundo e na coluna completa de água. As medições destas variáveis nas diferentes profundidades são devido ao fato que elas poderiam apresentar um comportamento diferente.

Devido a algumas análises feitas na "Universidad del Quindío" en um convênio com o INVEMAR, " Instituto de Investigações Marinas y Costeras José Benito Dias de Andreis", utilizando essas séries, surgiu-nos a suspeita de que algumas das séries poderiam estar sendo geradas por um mesmo processo estocástico e, conseqüentemente, fixada uma estação, não seria necessário fazer as medições nas diferentes profundidades. Dessa maneira surgiu o interesse de verificar a conjectura de que as séries são geradas pelo mesmo processo.

Nesta dissertação apresentamos as metodologias usadas por diferentes autores com o objetivo de testar a igualdade de séries temporais univariadas e multivariadas.

No Capítulo 2 apresentamos alguns conceitos básicos e definições preliminares. Nos Capítulos 3 e 4 apresentamos um pouco de teoria básica de séries temporais no enfoque espectral e temporal, respectivamente. No Capítulo 5 apresentamos os diferentes testes usados para comparar séries univariadas e multivariadas. Utilizando um enfoque temporal para comparar duas séries univariadas independentes, Quenouille (1958) propõe comparar as funções de autocorrelação das duas séries enquanto que Mélard e Roy (1984), propõem comparar as funções de autocovariância. Segundo um enfoque espectral, Coates e Diggle (1986) desenvolveram 
dois testes para comparar as funções de densidade espectral de duas séries independentes. Num mesmo enfoque espectral e, generalizando as idéias de Coates e Diggle (1986), Carmona e Wang (1996), desenvolveram testes para comparar duas séries temporais multivariadas, tanto para o caso independente quanto para o caso dependente, comparando as matrizes de densidade espectral. No Capítulo 6 apresentamos algumas simulações com o objetivo de analisar o poder dos testes apresentados no Capítulo 5 e de comparar o poder dos diferentes testes univariados. Finalmente, no Capítulo 7 aplicamos estes testes para verificar a conjectura de que salinidade e temperatura medidas na superfície e no fundo da estação BCG ("Boca de Caño Grande") da CGSM estão sendo geradas pelo mesmo processo. 


\section{Capítulo 2}

\section{Conceitos Básicos e Definições Preliminares}

Uma série temporal é um conjunto de observações $x_{t}$ onde cada observação é registrada num ponto $t$ sobre um conjunto dado (tempo, espaço,etc.). Quando as observações são feitas em intervalos discretos, a série se diz discreta (séries de nosso interesse), se as observações são feitas em tempos contínuos dizemos que a série é contínua.

Uma definição e tratamento mais formal de séries temporais encontra-se dentro da teoria dos processos estocásticos.

Definição 2.1 Seja $(\Omega, \mathfrak{A}, \mathfrak{\text { O}})$ um espaço de probabilidade e seja $T$ um conjunto de índices; um processo estocástico é uma função $X(t, \omega)$, definida em $T \mathrm{x} \Omega$, tal que para cada $t$ fixo $X(t, \omega)$ é uma variável aleatória sobre $(\Omega, \mathfrak{A}, \mathfrak{\Re})$ e, para cada $\omega$ fixo, $X(t, \omega)$ é uma trajetória, realização, função amostral do processo ou série temporal.

Assim, um processo estocástico é uma família de variáveis aleatórias $\{X(t, \cdot), t \in$ $T\}$, fixando $t$ ou uma família de trajetórias $\{X(\cdot, \omega), \omega \in \Omega\}$, fixando $\omega$. Mais específicamente, um processo estocástico é uma família de variáveis aleatórias indexadas em algum conjunto $T$ e definidas sobre um espaço amostral.

Daqui em diante suprimiremos a variável $\omega$ e simplesmente escreveremos $X(t, \omega)$ como $X(t)$ ou $X_{t}$. 
Um processo estocástico $\{X(t, \omega), t \in T, \omega \in \Omega\}$ fica bem definido quando conhecemos suas distribuições finito-dimensionais, ou seja, as distribuições das variáveis aleatórias $X_{t_{1}}, X_{t_{2}}, \ldots, X_{t_{n}}$ para qualquer inteiro positivo $n$.

Definição 2.2 A função de distribuição n-dimensional das variáveis aleatórias $\left\{X_{t_{1}}, X_{t_{2}}, \ldots, X_{t_{n}}\right\}$ é definida por

$$
F_{t_{1}, \ldots, t_{n}}\left(x_{t_{1}}, \ldots, x_{t_{n}}\right)=P\left(X\left(t_{1}\right) \leq x_{t_{1}}, \ldots, X\left(t_{n}\right) \leq x_{t_{n}}\right)
$$

para todo $n \geq 1$ e $t_{1}, \ldots, t_{n} \in \mathbf{T}$.

Definição 2.3 A série temporal $\left\{X_{t}, t \in \mathrm{T}\right\}$ é dita ser estritamente ou fortemente estacionária se as distribuições conjuntas de $\left(X_{t_{1}}, X_{t_{2}}, \ldots, X_{t_{k}}\right)$ 'e $\left(X_{t_{1}+h}, X_{t_{2}+h}, \ldots, X_{t_{k}+h}\right)$ 'são as mesmas para todo $t_{1}, t_{2}, \ldots, t_{k}, h \in \mathbf{T}$, ou seja, são invariantes sob translações no tempo. Assim,

$$
F\left(x_{t_{1}}, x_{t_{2}}, \ldots, x_{t_{k}}\right)=F\left(x_{t_{1}+h}, x_{t_{2}+h}, \ldots, x_{t_{k}+h}\right) \quad \forall t_{1}, t_{2}, \ldots, t_{k}, h \in \mathbf{T} .
$$

Definição 2.4 Dado o processo $\left\{X_{t}, t \in \mathbf{T}\right\}$ definimos

a função média do processo por

$$
\mu_{t}=E\left(X_{t}\right)=\int_{-\infty}^{\infty} X_{t} d F\left(X_{t}\right)
$$

a função variância do processo por

$$
\sigma_{t}^{2}=E\left(X_{t}-\mu_{t}\right)^{2}=\int_{-\infty}^{\infty}\left(X_{t}-\mu_{t}\right)^{2} d F\left(X_{t}\right)
$$

e a função de autocovariância entre duas variáveis $X_{t_{1}}$ e $X_{t_{2}}$ por $\gamma_{x}\left(t_{1}, t_{2}\right)=E\left(X_{t_{1}}-\mu_{t_{1}}\right)\left(X_{t_{2}}-\mu_{t_{2}}\right)=\int_{-\infty}^{\infty} \int_{-\infty}^{\infty}\left(X_{t_{1}}-\mu_{t_{1}}\right)\left(X_{t_{2}}-\mu_{t_{2}}\right) d F\left(X_{t_{1}}, X_{t_{2}}\right)$.

Considere um processo $\left\{X_{t}, t \in \mathrm{T}\right\}$ estritamente estacionário com $E X_{t}^{2}<$ $\infty$. Em particular, temos que $F_{X_{t_{k}}}\left(x_{t_{k}}\right)$ permanece invariante para todo $k \mathrm{e}$ $F_{X_{t_{1}}, X_{t_{2}}}\left(x_{t_{1}}, x_{t_{2}}\right)=F_{X_{t_{1}+k}, X_{t_{2}+k}}\left(x_{t_{1}}, x_{t_{2}}\right)$, implicando que

1. $E\left(X_{t}\right)=\mu_{t}=\mu, \quad \forall t \in \mathbf{T}$.

2. $\operatorname{Var}\left(X_{t}\right)=\sigma_{t}^{2}=\sigma, \quad \forall t \in \boldsymbol{\Gamma}$. 
3. $\gamma_{x}\left(t_{i}, t_{j}\right)=\gamma_{x}\left(\left|t_{i}-t_{j}\right|\right)=\gamma_{x}(k)$, para qualquer $t_{i}, t_{j}, k \in \mathbf{T}$, isto é, a covariância entre duas variáveis $X_{t_{i}}$ e $X_{t_{j}}$ só depende da sua defasagem $\left|t_{i}-t_{j}\right|=k$.

Definição 2.5 Se um processo estocástico $\left\{X_{t}, t \in \mathbf{T}\right\}$ satisfaz 1,2 e 3, dizemos que o processo é fracamente estacionário ou estacionário de segunda ordem ou ainda estacionário em sentido amplo.

Assim, um processo estritamente estacionário com segundo momento finito é fracamente estacionário.

Quando o processo $\{X(t), t \in \mathbf{T}\}$ é estacionário, a função de autocovariância $\gamma_{x}(k)$ satisfaz:

1. $\gamma_{x}(0)=\operatorname{Var}\left(X_{t}\right)>0$.

2. $\left|\gamma_{x}(k)\right| \leq \gamma_{x}(0), \quad \forall k \in \mathbf{Z}$.

3. $\gamma_{x}(-k)=\gamma_{x}(k)$.

4. $\gamma_{x}(k)$ é não negativa definida no sentido que

$$
\sum_{j=1}^{n} \sum_{l=1}^{n} a_{j} a_{l} \gamma_{x}\left(k_{j}-k_{l}\right) \geq 0
$$

para qualquer conjunto de pontos $k_{1}, \ldots, k_{n}$ e qualquer conjunto de escalares $a_{1}, \ldots, a_{n}$.

Definição 2.6 Uma seqüência de variáveis aleatórias $\left\{a_{t}\right\}$ é um processo ruído branco de média zero se

1. $E\left(a_{t}\right)=0 \quad \forall t \in \mathbf{T}$

2. $\gamma(k)=\left\{\begin{array}{ccc}\sigma_{a}^{2} & \text { para } & k=0 \\ 0 & \text { para } & k \neq 0\end{array}\right.$

ou seja sua esperança é zero, sua variância é constante e são variáveis aleatórias não correlacionadas.

Definição 2.7 O processo $\left\{X_{t}\right\}$ é Gaussiano se e somente se as funções de distribuição de $\left\{X_{t_{1}}, X_{t_{2}}, \ldots, X_{t_{k}}\right\}$, para qualquer $k$, são todas normais multivariadas. 
Se $\left\{X_{t}, t \in \mathbf{Z}\right\}$ é um processo Gaussiano fracamente estacionário então $\left\{X_{t}\right\}$ é estritamente estacionário, já que para todo $n \in\{1,2, \ldots\}$ e para todo $t_{1}, t_{2}, \ldots, h \in$ $\mathbf{Z}$, os vetores aleatórios $\left(X_{t_{1}}, X_{t_{2}}, \ldots, X_{t_{k}}\right)^{\prime}$ e $\left(X_{t_{1}+h}, X_{t_{2}+h}, \ldots, X_{t_{k}+h}\right)^{\prime}$ têm a mesma média e matriz de covariância, suficientes para determinar o processo.

Definição 2.8 Sejam $\mathrm{V}=\left(v_{1}, \ldots, v_{r}\right)$ um vetor $r$-dimensional de valores inteiros positivos, $\mathbf{X}=\left(X_{1}, \ldots, X_{r}\right)$ um vetor aleatório $r$-dimensional com $E\left|X_{j}\right|^{n}<$ $\infty, \quad j=1, \ldots, r$ onde os $X_{j}$ 's são reais ou complexos, com função característica dada por

$$
\varphi_{\mathbf{X}}\left(t_{1}, \ldots, t_{r}\right)=E\left(e^{i\left(t_{1} x_{1}+\ldots+t_{r} x_{r}\right)}\right) .
$$

Os cumulantes ou semi-invariantes do vetor $\mathrm{X}$ são dados pelo coeficiente de

$$
\frac{i^{v_{1}+\ldots+v_{r}}}{v_{1} ! \ldots v_{r} !} t_{1}^{v_{1}} \ldots t_{r}^{v_{r}}
$$

na expansão de Taylor de $\log \varphi_{\mathbf{X}}\left(t_{1}, \ldots, t_{r}\right)$, isto é,

$$
C_{\mathbf{X} v}=C u m\left(X_{1}^{v_{1}}, \ldots, X_{r}^{v_{r}}\right)=\left.\frac{\partial^{v_{1}+\ldots+v_{k}}}{\partial t_{1}^{v_{1}} \ldots \partial t_{r}^{v_{r}}} \log \varphi \mathbf{X}\left(t_{1}, \ldots, t_{r}\right)\right|_{|t|=0}
$$

onde $|t|=\left|t_{1}\right|+\ldots+\left|t_{r}\right| e$

$$
\log \varphi_{\mathbf{X}}\left(t_{1}, \ldots, t_{r}\right)=\sum_{v_{1}+\ldots+v_{r} \leq n} \frac{i^{v_{1}+\ldots+v_{r}}}{v_{1} ! \ldots v_{r} !} t_{1}^{v_{1}} \ldots t_{r}^{v_{r}} C_{\mathbf{X}^{\vee}}+O\left(|t|^{n}\right)
$$

é a expansão de Taylor de $\log \varphi_{\mathbf{x}}\left(t_{1}, \ldots, t_{r}\right)$.

\section{Resultado 1}

$$
C_{\mathrm{X} v}=\sum_{\lambda^{(1)}+\ldots+\lambda^{(q)}=\mathrm{V}} \frac{(-1)^{q-1}}{q !} \frac{\mathrm{V} !}{\lambda^{(1) !} \ldots \lambda^{(q)} !} \prod_{j=1}^{q} E\left(\mathbf{X}^{\lambda^{(j)}}\right)
$$

onde $\lambda^{(j)}, j=1, \ldots, q$, são vetores não negativos satisfazendo $\lambda^{(1)}+\ldots+\lambda^{(q)}=\mathrm{V}$. A operação $\mathbf{u}$ ! onde $\mathbf{u}$ é um vetor com componentes $\left(u_{1}, \ldots, u_{r}\right)$ é dada por $u_{1} ! \ldots u_{r} ! \mathrm{e} \mathbf{u}^{\mathrm{V}}=u_{1}{ }^{v_{1}} \ldots u_{r}{ }^{v_{r}}$. Logo $\sum_{\lambda^{(1)}+\ldots+\lambda^{(q)}=\mathrm{V}}$ indica a soma sobre todas as coleções ordenadas de vetores não negativos $\lambda^{(j)},\left|\lambda^{(j)}\right|>0$, que somam $\mathbf{V}$. 


\section{$\underline{\text { Resultado } 2}$}

Se $\mathrm{V}=(1, \ldots, 1), \quad C_{\mathbf{X}^{\vee}}=\operatorname{Cum}\left(X_{1}, \ldots, X_{r}\right)$ é chamado o cumulante simples do vetor $\mathrm{X}$ e é dado pelo coeficiente de $\mathrm{i}^{\mathrm{r}} \mathrm{t}_{\mathbf{1}} \ldots \mathrm{t}_{\mathbf{r}}$ na fórmula de expansão de Taylor (2.1) e, segundo o Resultado 1, temos que

$$
C_{\mathbf{X}^{\mathrm{v}}}=\sum_{\lambda(1)+\ldots+\lambda(q)=\mathrm{v}} \frac{(-1)^{q-1}}{q !} \prod_{j=1}^{q} E\left(\mathbf{X}^{\lambda^{(j)}}\right),
$$

para todo $q=1, \ldots, r$ tal que $\lambda^{(j)}, j=1, \ldots, q$ são vetores não negativos satisfazendo $\lambda^{(1)}+\ldots+\lambda^{(q)}=\mathrm{V}$.

As principais propriedades dos cumulantes são:

1. $\operatorname{Cum}\left(a_{1} X_{1}, \ldots, a_{r} X_{r}\right)=a_{1} \ldots a_{r} \operatorname{Cum}\left(X_{1}, \ldots, X_{r}\right)$ para constantes $a_{1}, \ldots, a_{r}$.

2. $\operatorname{Cum}\left(X_{1}, \ldots, X_{r}\right)$ é simétrico em seus argumentos.

3. $\operatorname{Cum}\left(X_{j}\right)=E X_{j}$ para $j=1, \ldots, r$.

4. $\operatorname{Cum}\left(X_{j}, \overline{X_{k}}\right)=\operatorname{Cov}\left(X_{j}, X_{k}\right)$ para $j, k=1, \ldots, r$, implicando que

$$
\operatorname{Cum}\left(X_{j}, \overline{X_{j}}\right)=\operatorname{Var}\left(X_{j}\right) .
$$

5. Se algum subconjunto das X's é independente das demais $X$ 's então

$$
\operatorname{Cum}\left(X_{1}, \ldots, X_{r}\right)=0 .
$$

6. Para o vetor aleatório $\left(Z_{1}, X_{1}, \ldots, X_{r}\right)$

$$
\operatorname{Cum}\left(X_{1}+Z_{1}, X_{2}, \ldots, X_{r}\right)=\operatorname{Cum}\left(X_{1}, X_{2}, \ldots, X_{r}\right)+\operatorname{Cum}\left(Z_{1}, X_{2}, \ldots, X_{r}\right) .
$$

7. Se os vetores aleatórios $\left(X_{1}, \ldots, X_{r}\right)$ e $\left(Z_{1}, \ldots, Z_{r}\right)$ são independentes então

$$
\operatorname{Cum}\left(X_{1}+Z_{1}, \ldots, X_{r}+Z_{r}\right)=\operatorname{Cum}\left(X_{1}, \ldots, X_{r}\right)+\operatorname{Cum}\left(Z_{1}, \ldots, Z_{r}\right) .
$$

Para maiores detalhes consultar Brillinger(1981), Leonov e Shiryaev(1959).

Definição 2.9 Dada a série temporal r-variada $\mathbf{X}(\mathbf{t})=\left\{X_{1}(t), \ldots, X_{r}(t)\right\}$, $t=0, \pm 1, \ldots$, satisfazendo $E\left|X_{j}(t)\right|^{k}<\infty$, a função

$$
C_{\mathbf{X}}\left(t_{1}, \ldots, t_{k}\right)=\operatorname{Cum}\left(X_{1}\left(t_{1}\right), \ldots, X_{k}\left(t_{k}\right)\right)
$$

é denominada a função cumulante conjunta de ordem $k$ da série $\mathbf{X}(\mathrm{t})$, $t=0, \pm 1, \ldots$. 
Definição 2.10 Considere a série temporal estacionária $X(t), \quad t=0, \pm 1, \ldots$ com função de autocovariância $\gamma_{x}(k)=\operatorname{Cov}\left(X_{t}, X_{t+k}\right), \quad t, k=0, \pm 1, \ldots$, satisfazendo

$$
\sum_{k=-\infty}^{\infty}\left|\gamma_{x}(k)\right|<\infty
$$

então o espectro de $X(t)$ na freqüência $\lambda$ existe e é dado por

$$
f_{x}(\lambda)=(2 \pi)^{-1} \sum_{k=-\infty}^{\infty} e^{-i \lambda k} \gamma_{x}(k), \quad-\infty<\lambda<\infty .
$$

Logo o espectro de $X(t)$ é a transformada de Fourier de $\gamma_{x}(k)$.

Demonstra-se que o espectro $f_{x}(\lambda)$ definido por (2.2) é uma função real, limitada, não negativa e uniformemente contínua. Também $f_{x}(\lambda)$ é par e periódica, de período $2 \pi$, com respeito a $\lambda$. Devido a estas duas últimas características, para conhecer completamente a função $f_{x}(\lambda)$ basta tomar um intervalo de comprimento $2 \pi$, por exemplo $[-\pi, \pi]$ e só utilizar $[0, \pi]$ como domínio fundamental na definição de $f_{x}(\lambda)$.

A relação (2.2) pode ser invertida e a função de autocovariância $\gamma_{x}(k)$ pode ser escrita como

$$
\gamma_{x}(k)=\int_{-\pi}^{\pi} e^{i \lambda k} f_{x}(\lambda) d \lambda, \quad k=0, \pm 1, \ldots
$$

No caso em que $k=0$ temos,

$$
\operatorname{Var}\left(X_{t}\right)=\gamma_{x}(0)=\int_{-\pi}^{\pi} f_{x}(\lambda) d \lambda
$$

indicando que o espectro pode ser interpretado como a decomposição da variância do processo $X(t)$, com $f_{x}(\lambda) d \lambda$ a contribuição à variância do processo devido à componente do processo com freqüências no intervalo $(\lambda, \lambda+d \lambda)$.

(2.2) e (2.3) formam um par de Fourier, implicando uma equivalência entre o espectro e a função de autocovariância quanto à informação probabilística que fornecem, na análise no domínio das freqüências ou no domínio do tempo, respectivamente.

Definição 2.11 a) Um vetor aleatório $r$-dimensional $\mathbf{X}=\left(X_{1}, \ldots, X_{r}\right)^{\prime}$ com componentes reais tem uma distribuição normal multivariada de dimensão 
$r$, com vetor de médias $\mu_{\mathbf{X}}=\left[\mu_{1}, \ldots, \mu_{r}\right]^{\prime}$ e matriz de covariância $\Sigma_{X X}$, se sua função de densidade é dada por

$$
f_{\mathbf{X}}\left(x_{1}, \ldots, x_{r}\right)=(2 \pi)^{-\frac{r}{2}}\left|\Sigma_{X X}\right|^{-1} e^{-\frac{1}{2}\left(\mathbf{X}-\mu_{\mathbf{X}}\right)^{\prime} \Sigma_{\mathbf{X}}^{-1}\left(\mathbf{X}-\mu_{\mathbf{X}}\right)}
$$

com notação $\mathrm{X} \sim N_{r}\left(\mu_{\mathrm{X}}, \Sigma_{\mathrm{XX}}\right)$.

b) Um vetor aleatório $r$-dimensional $\mathrm{X}=\left(X_{1}, \ldots, X_{r}\right)^{\prime}$ com componentes complexas da forma $X_{j}=R X_{j}+i I X_{j}{ }^{*}, \quad j=1, \ldots, r$, tem uma distribuição normal multivariada complexa de dimensão $r$, com vetor de médias $\mu_{\mathrm{X}}=$ $\left[\mu_{1}, \ldots, \mu_{r}\right]^{\prime}$ e matriz de covariância $\Sigma_{X X}$, se o vetor $2 r$-dimensional

$$
\left[\begin{array}{c}
R X \\
I X
\end{array}\right] \sim N_{2 r}\left(\left[\begin{array}{c}
R \mu_{\mathbf{X}} \\
I \mu_{\mathbf{X}}
\end{array}\right] ; \frac{1}{2}\left[\begin{array}{cc}
R \Sigma_{\mathbf{X X}} & -I \Sigma_{\mathbf{X X}} \\
I \Sigma_{\mathbf{X X}} & R \Sigma_{\mathbf{X X}}
\end{array}\right]\right)
$$

onde $\Sigma_{X X}$ é uma matriz de dimensão $r \times r$, Hermitiana não negativa definida.

Notação: $\mathbf{X} \sim \mathrm{N}_{r}^{c}\left(\mu_{\mathbf{X}}, \Sigma_{\mathbf{X X}}\right)$.

Se $\mathbf{X} \sim \mathrm{N}_{r}^{c}\left(\mu_{\mathbf{X}}, \Sigma_{\mathbf{X X}}\right)$ então temos as seguintes propriedades:

1. $E \mathbf{X}=\mu_{\mathbf{X}}$.

2. $E\left[\left(\mathbf{X}-\mu_{\mathbf{X}}\right) \overline{\left(\mathbf{X}-\mu_{\mathbf{X}}\right)^{\prime}}\right]=\Sigma_{X X}$.

3. $E\left[\left(\mathbf{X}-\mu_{\mathbf{X}}\right)\left(\mathbf{X}-\mu_{\mathbf{X}}\right)^{\prime}\right]=0$.

4. Se $\Sigma_{X X}$ é não singular então a função de densidade de $\mathbf{X}$ é dada por

$$
\begin{gathered}
f_{\mathbf{X}}\left(x_{1}, \ldots, x_{r}\right)=\pi^{-r}\left|\Sigma_{X X}\right|^{-1} e^{-\overline{\left(\mathbf{X}-\mu_{\mathbf{X}}\right)^{\prime}} \Sigma_{\mathbf{X} \mathbf{X}}^{-1}\left(\mathbf{X}-\mu_{\mathbf{X}}\right)} \\
\text { para }-\infty<R X_{j}<\infty \text { e }-\infty<I X_{j}<\infty .
\end{gathered}
$$

5. Se $\Sigma_{X X}$ é uma matriz diagonal então as componentes de $\mathbf{X}$ são estatisticamente independentes.

6. Quando $r=1$, se $X \sim N_{1}^{c}\left(\mu_{x}, \sigma_{x}\right)$ então $R X$ e $I X$ são variáveis aleatórias independentes com distribuições $\mathrm{N}_{1}\left(R \mu_{x}, \sigma_{x} / 2\right)$ e $\mathrm{N}_{1}\left(I \mu_{x}, \sigma_{x} / 2\right)$, respectivamente.

${ }^{*} R$ e $I$ indicam a parte real e imaginária de $X$ respectivamente 
Definição 2.12 a) Dizemos que a seqüência vetorial $\zeta_{\mathbf{T}}$ de dimensão $r, \quad \mathbf{T}=$ $1,2, \ldots$,é assintoticamente $N_{r}\left(\mu_{\mathbf{T}}, \Sigma_{\mathbf{T}}\right)$ se a seqüência $\Sigma_{\mathbf{T}}^{-1 / 2}\left(\zeta_{\mathbf{T}}-\mu_{\mathbf{T}}\right) \stackrel{D}{\longrightarrow} N_{r}(\mathbf{0}, \mathbf{I})$.

b) Dizemos que a seqüência vetorial $\zeta_{\mathbf{T}}$ de dimensão $r, \mathbf{T}=1,2, \ldots$ é assintoticamente $N_{r}^{c}\left(\mu_{\mathbf{T}}, \Sigma_{\mathbf{T}}\right)$ se a seqüência $\Sigma_{\mathbf{T}}^{-1 / 2}\left(\zeta_{\mathbf{T}}-\mu_{\mathbf{T}}\right) \stackrel{D}{\longrightarrow} N_{r}^{c}(\mathbf{0}, \mathbf{I})$.

Definição 2.13 a) A matriz de dimensão $r \times r$ das variáveis aleatórias

$$
\mathrm{W}=\sum_{j=1}^{n} X_{j} X_{j}^{\prime}
$$

tem uma distribuição Wishart de dimensão $r$ e graus de liberdade $n$ se $X_{1}, \ldots, X_{n}$ são independentes com distribuição normal $N_{r}\left(0, \Sigma_{\mathbf{X X}}\right)$.

A notação usada é $\mathbf{W} \sim W_{r}\left(n, \Sigma_{\mathbf{X x}}\right)$.

b) A matriz de dimensão $r \times \mathrm{r}$ das variáveis aleatórias

$$
\mathbf{W}=\sum_{j=1}^{n} X_{j} \overline{X_{j}^{\prime}}
$$

tem uma distribuição Wishart complexa de dimensão $r$ e graus de liberdade $n$ se $X_{1}, \ldots, X_{n}$ são independentes com distribuição normal complexa $N_{r}^{c}\left(\mathbf{0}, \Sigma_{\mathbf{X x}}\right)$.

A notação usada é $\mathrm{W} \sim W_{r}^{c}\left(n, \Sigma_{\mathbf{X x}}\right)$ e a função de densidade de $\mathrm{W}$ é dada por

$f_{\mathrm{W}}(\mathrm{w})=\left[\pi^{r(r-1) / 2} \prod_{j=1}^{n} \Gamma(n-j+1)\right]^{-1}\left|\Sigma_{X}\right|^{-n}|\mathbf{W}|^{n-r} e^{\left(-t r \Sigma_{X X}^{-1} \mathrm{~W}\right)}, \quad n \geq r \quad \mathbf{w} \geq 0$.

A distribuição de Wishart complexa tem as seguintes propriedades:

1. $\overline{\mathrm{W}}=\mathbf{W}^{\prime}$.

2. $E \mathbf{W}=n \Sigma_{\mathbf{X X}}$.

3. $\operatorname{Cov}\left(W_{j k}, W_{l m}\right)=E\left[\left(W_{j k}-n \Sigma_{j k}\right), \overline{\left(W_{l m}-n \Sigma_{l m}\right)}\right]=n \Sigma_{j l} \overline{\Sigma_{k m}}$.

4. Se $\mathrm{W} \sim W_{r}^{c}(n, \Sigma)$, então $W_{j j} \sim \Sigma_{j j} \chi_{2 n}^{2} / 2$.

5. Se $\mathbf{Y} \sim N_{n}^{c}\left(\mu, \sigma^{2} \mathbf{I}\right)$ e $\overline{\mathbf{Y}^{\prime}} \mathbf{Y}=\overline{\mathbf{Y}^{\prime}} \mathbf{A}_{1} \mathbf{Y}+\ldots+\overline{\mathbf{Y}^{\prime}} \mathbf{A}_{K} \mathbf{Y}$, onde $\mathbf{A}_{k}$ é uma matriz Hermitiana de posto $n_{k}, \quad k=1, \ldots, K$, então $\overline{\mathbf{Y}^{\prime}} \mathbf{A}_{1} \mathbf{Y}, \ldots, \overline{\mathbf{Y}^{\prime}} \mathbf{A}_{K} \mathbf{Y}$, 
são independentes e $\overline{\mathbf{Y}^{\prime}} \mathbf{A}_{k} \mathbf{Y}$ tem uma distribuição qui-quadrado não central dada por

$$
\sigma^{2} \frac{\chi_{2 n_{k}}^{2}\left(\frac{\overline{\mu^{\prime}} \mu}{\sigma^{2}}\right)}{2}
$$

se e somente se $n_{1}+\ldots+n_{K}=n$.

Para maiores detalhes consultar Brillinger(1981), Miller(1968), Kabe(1966), Srivastava(1965), Goodman(1966). 


\section{Capítulo 3}

\section{Análise Espectral de Processos Estacionários}

\subsection{Processos Univariados}

Definição 3.1 a) Considere uma amostra $X(0), X(1), \ldots, X(T-1)$ da seqüência $X(t), t=0, \pm 1, \ldots$ A transformada finita de Fourier destes valores é dada por

$$
d_{x}^{(T)}(\lambda)=\sum_{t=0}^{T-1} X(t) e^{-i \lambda t}, \quad-\infty<\lambda<\infty .
$$

Quando $T=2 n+1, n$ inteiro, (3.1) é equivalente a

$$
d_{x}^{(T)}(\lambda)=e^{-i \lambda n} \sum_{k=-n}^{n} X(k+n) e^{-i \lambda k}, \quad-\infty<\lambda<\infty .
$$

b) Se, em particular, $\lambda$ tomar os valores discretos $\lambda=\frac{2 \pi s}{T}, \quad s=0, \ldots, T-1$, então os valores

$$
d_{x}^{(T)}(\lambda)=d_{x}^{(T)}\left(\frac{2 \pi s}{T}\right), \quad s=0, \ldots, T-1
$$

constituem a transformada discreta de Fourier de $X(t), \quad t=0, \ldots, T-1$. O conjunto de coeficientes $\left\{\frac{2 \pi s}{T}\right\}$ denomina-se freqüências discretas de Fourier. 
A transformada discreta de Fourier satisfaz as seguintes propriedades:

1. $d_{x}^{(T)}(\lambda+2 \pi)=d_{x}^{(T)}(\lambda)$.

2. $\overline{d_{x}^{(T)}(\lambda)}=d_{x}^{(T)}(-\lambda)$ se $X(t)$ é real.

3. $d_{\alpha x+\beta y}^{(T)}(\lambda)=\alpha d_{x}^{(T)}(\lambda)+\beta d_{y}^{(T)}(\lambda)$, para escalares $\alpha$ e $\beta$.

A relação (3.1) pode ser invertida e $X(t)$ pode ser expresso por

$$
X(t)=(2 \pi)^{-1} \int_{0}^{2 \pi} d_{x}^{(T)}(\lambda) e^{i \lambda t} d \lambda, \quad t=0, \ldots, T-1
$$

ou, alternativamente,

$$
X(t)=T^{-1} \sum_{s=0}^{T-1} e^{i \frac{2 \pi s t}{T}} d_{x}^{(T)}\left(\frac{2 \pi s}{T}\right), \quad t=0, \ldots, T-1 .
$$

Os resultados apresentados a seguir podem ser vistos em Brillinger (1981).

Teorema 3.1 Se $\mathbf{X}(\mathrm{t})=\left(X_{1}(t), \ldots, X_{r}(t)\right)^{\prime} \quad t=0, \pm 1, \ldots$ é um processo $r$ variado e estritamente estacionário com função cumulante de ordem $k$ satisfazendo

$$
\sum_{u_{1}, \ldots, u_{k-1}=-\infty}^{\infty}\left|C_{\mathbf{X}}\left(u_{1}, \ldots, u_{k-1}\right)\right|<\infty
$$

e $s_{j}(T)$ um inteiro com $\lambda_{j}(T)=\frac{2 \pi s_{j}(T)}{T} \rightarrow \lambda_{j}$ quando $T \rightarrow \infty$ para $j=1,2, \ldots, J$, então

$$
d_{X}^{(T)}\left(\lambda_{j}(T)\right) \stackrel{D}{\longrightarrow} N_{r}^{c}\left(0,2 \pi T f_{X}\left(\lambda_{j}\right)\right)
$$

para $2 \lambda_{j}(T), \quad \lambda_{j}(T) \pm \lambda_{k}(T) \not \equiv 0(\bmod 2 \pi), \quad 1 \leq j<k \leq J, \quad T=1,2, \ldots$ Além disso, $\quad d_{X}^{(T)}\left(\lambda_{j}(T)\right)$ e $d_{X}^{(T)}\left(\lambda_{k}(T)\right)$ são independentes.

Segundo o Teorema (3.1) quando $r=1$ temos em particular que

$$
d_{x}^{(T)}(\lambda) \stackrel{\mathrm{D}}{\longrightarrow} N_{1}^{c}\left(0,2 \pi T f_{x}(\lambda)\right) \quad \text { se } \quad \lambda \not \equiv 0 \quad(\bmod \pi)
$$

o que sugere um possível estimador para $f_{x}(\lambda)$ dado por

$$
\begin{aligned}
I_{x}^{(T)}(\lambda) & =(2 \pi T)^{-1}\left|d_{x}^{(T)}(\lambda)\right|^{2} \\
& =(2 \pi T)^{-1}\left(\sum_{t=0}^{T-1} X(t) e^{-i \lambda t}\right)\left(\sum_{s=0}^{T-1} X(s) e^{i \lambda s}\right) .
\end{aligned}
$$

A estatística (3.3) é conhecida como o periodograma de segunda ordem, ou simplesmente, o periodograma da série $X(t)$. 
Teorema 3.2 Se $X(t), \quad t=0, \pm 1, \ldots$, é um processo estritamente estacionário satisfazendo

$$
\sum_{k=-\infty}^{\infty}\left|\gamma_{x}(k)\right|<\infty
$$

então

1. $E I_{x}^{(T)}(\lambda)=(2 \pi T)^{-1} \int_{-\pi}^{\pi}\left[\frac{\operatorname{sen} T(\lambda-\alpha) / 2}{\operatorname{sen}(\lambda-\alpha) / 2}\right]^{2} f_{x}(\alpha) d \alpha+(2 \pi T)^{-1}\left[\frac{\operatorname{sen} T \lambda / 2}{\operatorname{sen} \lambda / 2}\right]^{2} \mu_{x}^{2}$ e se $\lambda \not \equiv 0(\bmod 2 \pi)$

$$
\lim _{T \rightarrow \infty} E\left(I_{x}^{(T)}(\lambda)\right)=f_{x}(\lambda)
$$

ou seja, $I_{x}^{(T)}(\lambda)$ é um estimador assintoticamente não viciado de $f_{x}(\lambda)$.

2. Sob a hipótese adicional

$$
\begin{gathered}
\sum_{k=-\infty}^{\infty}|k|\left|\gamma_{x}(k)\right|<\infty \\
E I_{x}^{(T)}(\lambda)=f_{x}(\lambda)+(2 \pi T)^{-1}\left[\frac{\operatorname{sen} T \lambda / 2}{\operatorname{sen} \lambda / 2}\right]^{2} \mu_{x}^{2}+O\left(T^{-1}\right)
\end{gathered}
$$

onde o termo $O\left(T^{-1}\right)$ é uniforme em $\lambda$.

3. Quando $\omega=\frac{2 \pi r}{T}$ e $\lambda=\frac{2 \pi s}{T} \operatorname{com} r, s, r \pm s \not \equiv 0(\bmod T)$,

$$
\begin{gathered}
\operatorname{Var} I_{x}^{(T)}(\lambda)=f_{x}^{2}(\lambda)+O\left(T^{-1}\right), \\
\operatorname{Cov}\left(I_{x}^{(T)}(\lambda), I_{x}^{(T)}(\omega)\right)=O\left(T^{-1}\right) .
\end{gathered}
$$

4. $I_{x}^{(T)}\left(\lambda_{j}(T)\right) \stackrel{D}{\longrightarrow} f_{x}\left(\lambda_{j}\right) \chi_{2}^{2} / 2 \quad$ para $2 \lambda_{j}(T), \quad \lambda_{j}(T) \pm \lambda_{k}(T) \not \equiv 0(\bmod 2 \pi)$, $1 \leq j<k \leq J e T=1,2, \ldots$ Além disso, $I_{x}^{(T)}\left(\lambda_{j}(T)\right)$ e $I_{x}^{(T)}\left(\lambda_{k}(T)\right)$ são independentes.

Os resultados acima indicam que o periodograma é um estimador assintoticamente não viciado, embora inconsistente, para o espectro $f_{x}(\lambda)$. Um estimador com melhores propriedades é definido a seguir. 
Definição 3.2 O estimador suavizado de periodogramas para $f_{x}(\lambda)$ é dado por

$$
f_{x}^{(T)}(\lambda)=\sum_{j=-m}^{m} W_{T}(j) I_{x}^{(T)}\left(\frac{2 \pi[s(T)+j]}{T}\right) \text { se } \lambda \not \equiv 0 \quad(\bmod \pi)
$$

onde $\frac{s(T)}{T} \rightarrow \lambda$ quando $T \rightarrow \infty$ e $W_{T}(\cdot)$ é denominada janela espectral com as seguintes propriedades:

- $m \rightarrow \infty$ e $\frac{m}{T} \rightarrow 0$ quando $T \rightarrow \infty$.

- $W_{T}(j)=W_{T}(-j)$ e $W_{T}(j) \geq 0, \quad \forall j$.

- $\sum_{j=-m}^{m} W_{T}(j)=1$.

- $\sum_{j=-m}^{m} W_{T}^{2}(j) \rightarrow 0$ quando $T \rightarrow \infty$.

Teorema 3.3 Seja $X(t), \quad t=0, \pm 1, \ldots$, uma série estritamente estacionária satisfazendo

$$
\sum_{k=-\infty}^{\infty}\left|\gamma_{x}(k)\right|<\infty
$$

então

1. $f_{x}^{(T)}(\lambda)$ é um estimador consistente para $f_{x}(\lambda)$.

2. $f_{x}^{(T)}\left(\lambda_{j}(T)\right) \stackrel{D}{\longrightarrow} f_{x}\left(\lambda_{j}\right) \chi_{4 m+2}^{2} /(4 m+2)$ independentes, se $s_{j}(T)$ é um inteiro tal que $\frac{2 \pi s_{j}(T)}{T} \rightarrow \lambda_{j}$, quando $T \rightarrow \infty e 2 \lambda_{j}(T), \lambda_{j}(T) \pm \lambda_{k}(T) \not \equiv 0(\bmod 2 \pi)$ para $1 \leq j<k \leq J$.

Para maiores detalhes ver Brillinger(1981) e Priestley(1981).

\subsection{Processos Multivariados}

Definição 3.3 Dados dois processos $\left\{X_{t}\right\}$ e $\left\{Y_{t}\right\}, t=0, \pm 1, \pm 2, \ldots$, a função de covariância cruzada entre $\left\{X_{t}\right\}$ e $\left\{Y_{t}\right\}$ é dada por

$$
\gamma_{x y}(k)=E\left(X_{t}-\mu_{x}\right)\left(Y_{t+k}-\mu_{y}\right), \quad k=0, \pm 1, \ldots
$$


A função de covariância cruzada tem as seguintes propriedades:

1. $\gamma_{x y}(k) \neq \gamma_{x y}(-k)$.

2. $\gamma_{x y}(k)=\gamma_{y x}(-k)$.

Definição 3.4 Se $\left\{X_{t}\right\}$ e $\left\{Y_{t}\right\}, t=0, \pm 1, \pm 2, \ldots$, são dois processos conjuntamente estacionários com função de covariância cruzada $\gamma_{x y}(k)$ satisfazendo $\sum_{k=-\infty}^{\infty}\left|\gamma_{x y}(k)\right|<\infty$, então pode-se definir o espectro cruzado entre $\left\{X_{t}\right\}$ $e\left\{Y_{t}\right\}$ por

$$
f_{x y}(\lambda)=\frac{1}{2 \pi} \sum_{k=-\infty}^{\infty} \gamma_{x y}(k) e^{-i \lambda k}, \quad-\infty<\lambda<\infty .
$$

Observe que $f_{x y}(\lambda)$ é complexo já que $\gamma_{x y}(k) \neq \gamma_{x y}(-k)$.

Definição 3.5 Se $\left\{X_{t}\right\}$ e $\left\{Y_{t}\right\}, t=0, \pm 1, \pm 2, \ldots$, são dois processos conjuntamente estacionários com funções de densidade espectral $f_{x}(\lambda)$ e $f_{y}(\lambda)$, respectivamente, e espectro cruzado $f_{x y}(\lambda)$, a função de coerência quadrática entre $\left\{X_{t}\right\}$ e $\left\{Y_{t}\right\}$ é dada por

$$
R^{2}(\lambda)=\frac{\left|f_{x y}(\lambda)\right|^{2}}{f_{x}(\lambda) f_{y}(\lambda)}, \quad-\infty<\lambda<\infty .
$$

Observe que a função de coerência quadrática é real e satisfaz $0 \leq R^{2}(\lambda) \leq 1$.

Definição 3.6 Seja $\mathbf{X}(\mathbf{t})=\left[X_{1 t}, X_{2 t}, \ldots, X_{r t}\right]^{\prime}$ um processo $r$-dimensional conjuntamente estacionário, com vetor de médias $\mu=\left[\mu_{1}, \mu_{2}, \ldots, \mu_{r}\right]^{\prime}$ onde $E X_{i t}=$ $\mu_{i}$ é constante para cada $i=1, \ldots, r$ e a covariância cruzada entre $X_{i t}$ e $X_{j s}$ para todo $i, j=1, \ldots, r$ só depende da diferença $(s-t)$, então dizemos que $\Gamma_{\mathbf{X}}(\mathbf{k})$ é a função matricial de autocovariância de $X(t)$ se

$$
\begin{aligned}
\Gamma_{\mathbf{X}}(k) & =\operatorname{Cov}\left(\mathbf{X}_{t}, \mathbf{X}_{t+k}\right) \\
& =E\left[\left(\mathbf{X}_{t}-\mu\right)\left(\mathbf{X}_{t+k}-\mu\right)^{\prime}\right]
\end{aligned}
$$




$$
\begin{aligned}
\Gamma_{\mathbf{X}}(k)= & E\left[\begin{array}{c}
X_{1 t}-\mu_{1} \\
X_{2 t}-\mu_{2} \\
\vdots \\
X_{r t}-\mu_{r}
\end{array}\right]\left[X_{1 t+k}-\mu_{1}, X_{2 t+k}-\mu_{2}, \ldots, X_{r t+k}-\mu_{r}\right] \\
= & {\left[\begin{array}{cccc}
\gamma_{11}(k) & \gamma_{12}(k) & \ldots & \gamma_{1 r}(k) \\
\gamma_{21}(k) & \gamma_{22}(k) & \ldots & \gamma_{2 r}(k) \\
\vdots & \vdots & \ddots & \vdots \\
\gamma_{r 1}(k) & \gamma_{r 2}(k) & \ldots & \gamma_{r r}(k)
\end{array}\right] }
\end{aligned}
$$

para $k=0, \pm 1, \pm 2, \ldots$.

Algumas propriedades importantes são:

1. Se $i=j, \gamma_{i i}(k)$ é a função de autocovariância de $X_{i t}$, a i-ésima componente do processo $\mathbf{X}(\mathrm{t})$.

2. $\Gamma_{\mathbf{X}}(0)$ é a matriz de variâncias e covariâncias do processo $\mathbf{X}(\mathbf{t})$.

3. $\Gamma_{\mathbf{X}}(k)$ é positiva semidefinida no sentido que $\sum_{i=1}^{n} \sum_{j=1}^{n} \alpha_{i}^{\prime} \Gamma_{\mathbf{X}}\left(k_{i}-k_{j}\right) \alpha_{j} \geq 0$ para qualquer conjunto de pontos $k_{1}, \ldots, k_{n}$ e qualquer conjunto de vetores $\alpha_{1}, \ldots, \alpha_{n}$.

4. $\Gamma_{\mathbf{X}}(k)=\Gamma_{\mathbf{X}}^{\prime}(-k)$.

Teorema 3.4 Se a função matricial de covariâncias é absolutamente somável no sentido que cada uma das $r \times \mathrm{r}$ seqüências $\gamma_{i j}(k)$ seja absolutamente somável, então o espectro matricial ou função matricial de densidade espectral existe e é dado por

$$
\mathbf{f}_{\mathbf{X}}(\lambda)=\frac{1}{2 \pi} \sum_{k=-\infty}^{\infty} \Gamma_{\mathbf{X}}(k) e^{-i \lambda k}, \quad-\infty<\lambda<\infty .
$$

As principais propriedades de $\mathbf{f}_{\mathbf{X}}(\lambda)$ são:

1. O elemento $f_{i i}(\lambda)$ em (3.6) é o espectro da componente $X_{i t}$ de $\mathbf{X}(\mathbf{t})$.

2. $f_{i j}(\lambda)$ é o espectro cruzado entre as componentes $X_{i t}$ e $X_{j t}$ de $\mathbf{X}(\mathrm{t})$. 
3. $\mathbf{f}_{\mathbf{X}}(\lambda)$ é positiva semidefinida.

4. $\mathbf{f}_{\mathbf{X}}(\lambda)=\mathbf{f}_{\mathbf{X}}^{*}(\lambda)=\overline{\mathbf{f}_{\mathbf{X}}(\lambda)^{\prime}}$, ou seja, $\mathbf{f}_{\mathbf{X}}(\lambda)$ é uma matriz Hermitiana.

5. $\mathbf{f}_{\mathbf{X}}(\lambda)$ é periódica, de período $2 \pi$, com respeito a $\lambda$.

A relação (3.6) também pode ser invertida, de modo que

$$
\Gamma_{\mathbf{X}}(k)=\int_{-\pi}^{\pi} e^{i \lambda k} \mathbf{f}_{\mathbf{X}}(\lambda) d \lambda, \quad k=0, \pm 1, \ldots
$$

Definição 3.7 A tranformada finita de Fourier dos valores $\mathrm{X}(\mathrm{t}), t=0, \pm 1, \ldots$, $T-1$ de uma série $r$-dimensional com média $\mu_{\mathrm{X}}$ e matriz de densidade espectral $\mathbf{f}_{\mathbf{X}}(\lambda)$, é dada por

$$
d_{\mathbf{X}}^{(T)}(\lambda)=\left[d_{j}^{(T)}(\lambda)\right]=\left[\sum_{t=0}^{T-1} X_{j}(t) e^{-i \lambda t}\right], \quad-\infty<\lambda<\infty, \quad j=1, \ldots, r .
$$

Do Teorema (3.1) temos que

$$
d_{\mathbf{X}}^{(T)}(\lambda) \stackrel{\mathrm{D}}{\longrightarrow} N_{r}^{c}\left(0,2 \pi T f_{\mathbf{X}}(\lambda)\right) \quad \text { se } \quad \lambda \not \equiv 0 \quad(\bmod \pi) .
$$

Este resultado sugere como estimador de $\mathbf{f}_{\mathbf{X}}(\lambda)$, no caso $\lambda \not \equiv 0, \pm 2 \pi, \ldots$, a estatística

$$
I_{\mathrm{X}}^{(T)}(\lambda)=\left[I_{i j}^{(T)}(\lambda)\right]=\left[(2 \pi)^{-1} d_{i}^{(T)}(\lambda) \overline{d_{j}^{(T)}(\lambda)}\right]
$$

$\operatorname{com} \lambda=\frac{2 \pi s}{T}, s=1, \ldots,\left[\frac{T-1}{2}\right], \quad i, j=1, \ldots, r$.

As entradas da matriz $I_{\mathbf{X}}^{(T)}(\lambda)$ correspondem aos periodogramas e periodogramas cruzados de segunda ordem de $\{\mathbf{X}(t)\}$.

Teorema 3.5 Seja $\mathbf{X}(\mathbf{t}), t=0, \pm 1, \ldots$, uma série $r$-variada estacionária com vetor de médias $\mu_{\mathbf{X}}$ e função matricial de autocovariâncias $\Gamma_{\mathbf{X}}(k), \quad k=0, \pm 1, \pm 2, \ldots$, satisfazendo

$$
\sum_{k=-\infty}^{\infty}\left|\Gamma_{\mathbf{X}}(k)\right|<\infty
$$

então

1. $\lim _{T \rightarrow \infty} E I_{\mathbf{X}}^{(T)}(\lambda)=\mathbf{f}_{\mathbf{X}}(\lambda)$ se $\lambda \not \equiv 0(\bmod 2 \pi)$ ou se $\mu_{\mathbf{X}}=\mathbf{0}$. 
2. $\lim _{T \rightarrow \infty} \operatorname{Cov}\left(I_{i j}^{(T)}(\lambda), I_{i^{\prime} j^{\prime}}^{(T)}(\omega)\right)$

$$
\begin{aligned}
& =\eta(\lambda-\omega) f_{i i^{\prime}}(\lambda) f_{j j^{\prime}}(-\lambda)+\eta(\lambda+\omega) f_{i j^{\prime}}(\lambda) f_{j i^{\prime}}(-\lambda) \\
& =f^{\prime}(\lambda, \omega) \quad \text { para } \lambda, \omega \not \equiv 0 \quad(\bmod 2 \pi),
\end{aligned}
$$

$\leftrightarrow$ onde $\eta(\cdot)$ é a extensão periódica $2 \pi$ da função delta Dirac.

Assim, $I_{\mathbf{X}}^{(T)}(\lambda)$ também é assintoticamente não viciado embora inconsistente.

Teorema 3.6 Sob as mesmas condições do Teorema 3.1, assintoticamente, temos que

$$
I_{\mathbf{X}}^{(T)}\left(\lambda_{j}(T)\right) \stackrel{D}{\longrightarrow} W_{1}^{c}\left(1, \mathbf{f}_{\mathbf{X}}\left(\lambda_{j}\right)\right)
$$

para $j=1, \ldots, J, \quad 2 \lambda_{j}(T), \lambda_{j}(T) \pm \lambda_{k}(T) \not \equiv 0(\bmod 2 \pi) \quad$ para $\quad 1 \leq j<k \leq$ J. Além disso, $I_{\mathbf{X}}^{(T)}\left(\lambda_{j}(T)\right)$ e $I_{\mathbf{X}}^{(T)}\left(\lambda_{k}(T)\right)$ são assintoticamente independentes.

O Teorema 3.6 sugere um estimador para a matriz de densidade espectral $\mathbf{f}_{\mathbf{X}}(\lambda)$ dado por

$$
\mathrm{f}_{\mathbf{X}}{ }^{(T)}(\lambda)=(2 m+1)^{-1} \sum_{s=-m}^{m} I_{\mathbf{X}}^{(T)}\left(\frac{2 \pi[s(T)+s]}{T}\right) \quad \text { se } \quad \lambda \not \equiv 0 \quad(\bmod \pi)
$$

e denominado estimador suavizado da matriz de densidade espectral.

Como no caso univariado, este estimador faz parte de uma classe mais geral de estimadores consistentes da matriz de densidade espectral, definidos de forma similar a (3.5).

Teorema 3.7 Seja $\{\mathbf{X}(\mathbf{t})\}, t=0, \pm 1, \ldots$ uma série $r$-vetorial com vetor de médias $\mu_{\mathbf{X}}$ e satisfazendo

$$
\sum_{k=-\infty}^{\infty}\left|\Gamma_{\mathbf{X}}(k)\right|<\infty
$$

Se $\frac{2 \pi s(T)}{T} \rightarrow \lambda, T \rightarrow \infty$, então

$$
\lim _{T \rightarrow \infty} E \mathbf{f}_{\mathbf{X}}{ }^{(T)}(\lambda)=\mathbf{f}_{\mathbf{X}}(\lambda), \quad-\infty<\lambda<\infty .
$$


Teorema 3.8 Sob as condições do Teorema 3.6, seja $\mathbf{f}_{\mathbf{X}}{ }^{(T)}(\lambda)$ dado por (3.8) com $\lambda-\frac{2 \pi s(T)}{T}=O\left(T^{-1}\right)$, então

1. $\operatorname{Cov}\left(f_{i j}^{(T)}(\lambda), f_{i^{\prime} j^{\prime}}^{(T)}(\omega)\right)=\frac{f^{\prime}(\lambda, \omega)}{2 m+1}+O\left(T^{-1}\right) \quad$ se $\quad \lambda, \omega \neq 0 \quad(\bmod \pi)$ para $-\infty<\lambda, \omega<\infty$ e $f^{\prime}(\lambda, \omega)$ definida no Teorema 3.5.

2. $\mathbf{f}_{\mathbf{X}}^{(T)}(\lambda) \stackrel{D}{\longrightarrow}(2 m+1)^{-1} W_{r}^{c}\left(2 m+1, \mathbf{f}_{\mathbf{X}}(\lambda)\right)$ se $\lambda \not \equiv 0(\bmod \pi)$ onde $\mathbf{f}_{\mathbf{X}}{ }^{(T)}\left(\lambda_{j}\right), \quad j=1, \ldots, J, \quad$ são assintoticamente independentes se $\lambda_{j} \pm \lambda_{k} \not \equiv 0(\bmod 2 \pi) \quad$ para $\quad 1 \leq j<k \leq J$.

As demonstrações dos teoremas encontram-se em Brillinger(1981).

Para maiores detalhes consultar também Priestley(1981), Bloomfield(1976), Morettin(1979), Brockwell and Davis(1991). 


\section{Capítulo 4}

\section{Análise Temporal de Processos Estacionários}

\subsection{Processos Univariados}

Definição 4.1 Seja $\left\{\mathbf{X}_{\mathbf{t}}, t=0, \pm 1, \ldots\right\}$ uma série temporal estacionária, sua função de autocorrelação (fac) é definida por

$$
\rho_{x}(k)=\operatorname{Corr}\left(X_{t}, X_{t+k}\right)=\frac{\gamma_{x}(k)}{\gamma_{x}(0)}, \quad k \in \mathbf{Z}
$$

A função de autocorrelação satisfaz as seguintes propriedades:

1. $\rho_{x}(0)=1$.

2. $\left|\rho_{x}(k)\right| \leq 1, \quad \forall k \in \mathbf{Z}$.

3. $\rho_{x}(-k)=\rho_{x}(k)$.

4. $\rho_{x}(k)$ é uma função não negativa definida no sentido que

$$
\sum_{j=1}^{n} \sum_{l=1}^{n} a_{j} a_{l} \rho_{x}\left(k_{j}-k_{l}\right) \geq 0
$$

para qualquer conjunto de pontos $k_{1}, \ldots, k_{n}$ e qualquer conjunto de escalares $a_{1}, \ldots, a_{n}$. 
Definição 4.2 A função de autocovariância amostral da série observada $\left\{X_{1}, \ldots, X_{T}\right\}$ é dada por

$$
\widehat{\gamma}_{x}(k)=\frac{1}{T} \sum_{t=1}^{T-k}\left(X_{t+k}-\bar{X}\right)\left(X_{t}-\bar{X}\right), \quad 0 \leq k \leq T-1
$$

$\operatorname{com} \bar{X}=\frac{1}{T} \sum_{t=1}^{T} X_{t}$ e é tal que $\widehat{\gamma}_{x}(k)=\widehat{\gamma}_{x}(-k)$.

Conseqüentemente a função de autocorrelação amostral é dada por

$$
\widehat{\rho}(k)=\frac{\widehat{\gamma}_{x}(k)}{\widehat{\gamma}_{x}(0)}, \quad 0 \leq k \leq T-1 .
$$

A função de autocorrelação entre $X_{t}$ e $X_{t+k}$ tem a característica de estar influenciada pelas variáveis intermediárias $X_{t+1}, \ldots, X_{t+k-1}$. Uma função que estabelece a relação pura entre $X_{t}$ e $X_{t+k}$ eliminando o efeito das $k-1$ variáveis intermediárias $X_{t+1}, \ldots, X_{t+k-1}$ é a função de autocorrelação parcial.

Definição 4.3 A função de autocorrelação parcial $\Phi_{x}(k)$ de uma série temporal estacionária $\left\{X_{t}\right\}$ é dada por

$\Phi_{x}(k)=\left\{\begin{array}{l}\operatorname{Corr}\left(X_{t}, X_{t+1}\right)=\rho_{x}(1), \quad k=1 \\ \operatorname{Corr}\left(X_{t+k}-P_{\overline{s p}\left\{1, X_{t+1}, \ldots, X_{t+k-1}\right\}} X_{t+k}, X_{t}-P_{\overline{s p}\left\{1, X_{t+1}, \ldots, X_{t+k-1}\right\}} X_{t}\right), k \geq 2\end{array}\right.$

onde o termo $P_{\overline{s p}\left\{1, Y_{1}, \ldots, Y_{n}\right\}} Z$ indica a projeção do vetor $Z$ sobre o espaço gerado pelos vetores $Y_{1}, \ldots, Y_{n}$ e é tal que

$$
P_{\overline{s p}\left\{1, Y_{1}, \ldots, Y_{n}\right\}} Z=\sum_{i=0}^{n} \phi_{i} Y_{i}
$$

com $Y_{0}=1$ e onde $\phi_{0}, \ldots, \phi_{n}$ satisfazem $\left\langle\sum_{i=0}^{n} \phi_{i} Y_{i}, Y_{j}\right\rangle=\left\langle Z, Y_{j}\right\rangle^{*}$, $j=0,1, \ldots, n$ ou, também, $\quad \sum_{i=0}^{n} \phi_{i} E\left(Y_{i} Y_{j}\right)=E\left(Z Y_{j}\right), \quad j=0, \ldots, n$.

O teorema da projeção garante que a solução $\left(\phi_{0}, \ldots, \phi_{n}\right)$ existe.

Logo a função de autocorrelação parcial $\Phi_{x}(k)$, para $k \geq 2$, é a correlação dos dois resíduos obtidos depois de ajustar $X_{t+k}$ e $X_{t}$ sobre as variáveis intermediárias $X_{t+1}, \ldots, X_{t+k-1}$.

Uma outra definição é a seguinte.

* $\langle X, Y\rangle$ indica o produto interno entre os vetores $X$ e $Y$. 
Considere que $\left\{X_{t}\right\}$ é um processo estacionário de média zero, com função de autocovariância $\gamma_{x}(h)$ e tal que $\gamma_{x}(h) \rightarrow 0$ quando $h \rightarrow \infty$ e suponha que $\phi_{k j}, \quad j=1, \ldots, k ; k=1,2, \ldots$, são os coeficientes na representação

$$
P_{\overline{s p}\left\{X_{t}, \ldots, X_{t+k-1}\right\}} X_{t+k}=\sum_{j=1}^{k} \phi_{k j} X_{t+k-j} .
$$

Calculando as esperanças nas equações

$$
\left\langle X_{t+k}-P_{\overline{s p}\left\{X_{t}, \ldots, X_{t+k-1}\right\}} X_{t+k}, X_{t+k-j}\right\rangle=0, j=1, \ldots, k
$$

obtemos o sistema matricial

$$
\left[\begin{array}{ccccc}
\rho(0) & \rho(1) & \rho(2) & \ldots & \rho(k-1) \\
\rho(1) & \rho(0) & \rho(1) & \ldots & \rho(k-2) \\
\vdots & \vdots & \vdots & \ddots & \vdots \\
\rho(k-1) & \rho(k-2) & \rho(k-3) & \ldots & \rho(0)
\end{array}\right]\left[\begin{array}{c}
\phi_{k 1} \\
\phi_{k 2} \\
\vdots \\
\phi_{k k}
\end{array}\right]=\left[\begin{array}{c}
\rho(1) \\
\rho(2) \\
\vdots \\
\rho(k)
\end{array}\right] \quad k \geq 1
$$

Assim, a função de autocorrelação parcial $\Phi(k)$ de $\left\{X_{t}\right\}$ no "lag $k$ " é a solução de (4.1) dada por

$$
\Phi(k)=\phi_{k k}, \quad k \geq 1 .
$$

O sistema de equações (4.1) é conhecido como as equações de Yule-Walker.

Definição 4.4 A função de autocorrelação parcial amostral, na defasagem $k$, de $\left\{X_{1}, \ldots, X_{T}\right\}, \quad \widehat{\Phi}_{x}(k)$, é definida por

$$
\widehat{\Phi}_{x}(k)=\widehat{\phi}_{k k}, \quad 1 \leq k<T
$$

onde $\widehat{\Phi}_{x}(k)$ é unicamente determinado por (4.1) com cada $\rho(j)$ sustituído pela correspondente autocorrelação amostral $\hat{\rho}(j)$.

Teorema 4.1 Seja a série temporal $\left\{X_{t}\right\}$ tal que $X_{t}-\mu=\sum_{j=-\infty}^{\infty} \psi_{j} a_{t-j}$ onde a seqüência $\left\{\psi_{j}\right\}$ é absolutamente somável e os $a_{t}$ são variáveis aleatórias independentes de média 0 , variância constante $\sigma_{a}^{2}$ e com $E\left(a_{t}^{4}\right)=\xi \sigma^{4}<\infty$, então para $p$ e $q$ fixos $(p \geq q \geq 0)$

$$
\begin{aligned}
\lim _{T \rightarrow \infty}(T-q) \operatorname{Cov}\left(\widehat{\gamma_{x}}(p), \widehat{\gamma_{x}}(q)\right)= & (\xi-3) \gamma_{x}(p) \gamma_{x}(q)+\sum_{k=-\infty}^{\infty}\left[\gamma_{x}(k) \gamma_{x}(k-p+q)+\right. \\
& \left.+\gamma_{x}(k+q) \gamma_{x}(k-p)\right]
\end{aligned}
$$


Se o processo é normal $\xi=3$ e

$\lim _{T \rightarrow \infty}(T-q) \operatorname{Cov}\left(\widehat{\gamma_{x}}(p), \widehat{\gamma_{x}}(q)\right)=\sum_{k=-\infty}^{\infty}\left[\gamma_{x}(k) \gamma_{x}(k-p+q)+\gamma_{x}(k+q) \gamma_{x}(k-p)\right]$

Teorema 4.2 Sob as condições do Teorema 4.1 temos que, assintoticamente, para qualquer $h \in\{0,1,2, \ldots\}$,

$$
\widehat{\gamma_{x}}(h) \sim N_{h+1}\left(\gamma_{x}(h), T^{-1} \mathbf{V}\right)
$$

onde $\widehat{\gamma_{x}}(h)^{\prime}=\left[\widehat{\gamma_{x}}(0), \widehat{\gamma_{x}}(1), \ldots, \widehat{\gamma_{x}}(h)\right]$ e $\mathrm{V}$ é a matriz de covariâncias com elementos dados por (4.3) para $p, q=0, \ldots, h$.

Ver demonstração dos Teoremas 4.1 e 4.2 em Brockwell-Davis (1991).

\subsubsection{Processos Lineares}

Teorema 4.3 Se a seqüência de números reais $\left\{\psi_{j}\right\}$ e a seqüência de variáveis aleatórias $\left\{a_{j}\right\}$ satisfazem $\sum_{j=-\infty}^{\infty}\left|\psi_{j}\right|<\infty$ e $E\left(a_{t}^{2}\right) \leq k, t=0, \pm 1, \pm 2, \ldots$, para algum $k$ finito, então existe uma seqüência de variáveis aleatórias $\left\{X_{t}\right\}$ tal que para $t=0, \pm 1, \pm 2, \ldots$, satisfaz

$$
E\left\{\left|X_{t}-\sum_{j=-n}^{n} \psi_{j} a_{t-j}\right|^{2}\right\} \rightarrow 0 \text { para } T \rightarrow \infty .
$$

O resultado acima indica que $\sum_{j=-n}^{n} \psi_{j} a_{t-j}$ converge em média quadrática e, portanto, em probabilidade a $X_{t}$ e $E\left\{X_{t}^{2}\right\}<\infty$. Mais ainda, a seqüência de variáveis aleatórias $\left\{X_{t}\right\}$ é unicamente determinada exceto sobre um evento de probabilidade zero. Se além disso, $\left\{a_{t}\right\}$ é uma seqüência de variáveis aleatórias independentes, então

$$
\sum_{j=-n}^{n} \psi_{j} a_{t-j} \stackrel{\text { q.c. }}{\longrightarrow} X_{t}
$$

Ver demonstração do Teorema 4.3 em Fuller(1976).

Sem perda de generalidade e para simplificar a notação, daqui em diante, assumiremos que o processo $\left\{X_{t}\right\}$ tem média zero. 
Definição $4.5\left\{X_{t}\right\}$ é dito ser um processo linear geral se pode ser expresso na forma

$$
X_{t}=\sum_{j=0}^{\infty} \psi_{j} a_{t-j}
$$

onde $\left\{a_{t}\right\}$ é um processo puramente aleatório $e\left\{\psi_{j}\right\}$ é uma seqüência de constantes satisfazendo $\sum_{j=0}^{\infty} \psi_{j}^{2}<\infty$. Esta última condição garante que $\left\{X_{t}\right\}$ tenha variância finita e também que o processo (4.4) seja estacionário.

Para maiores detalhes ver Priestley(1981).

Observe que (4.4) é um caso particular do Teorema 4.3 onde $\psi_{j}=0$ para todo $j<0$.

Uma forma mais simplificada de representar (4.4) é através da utilização do operador de defasagem $B$, isto é,

$$
\begin{aligned}
X_{t} & =a_{t}+\psi_{1} a_{t-1}+\psi_{2} a_{t-2}+\ldots \\
& =\left(1+\psi_{1} B+\psi_{2} B^{2}+\ldots\right) a_{t} \\
& =\sum_{j=0}^{\infty}\left(\psi_{j} B^{j}\right) a_{t} \\
& =\Psi(B) a_{t}
\end{aligned}
$$

onde $\psi_{0}=1, \Psi(B)=\sum_{j=0}^{\infty} \psi_{j} B^{j}$ é a função geradora dos pesos $\psi_{j}$ e o operador $B$ é tal que $B^{d} X_{t}=X_{t-d}, d \geq 0$.

$\mathrm{O}$ processo linear (4.5) satisfaz as seguintes propriedades:

1. $\gamma_{x}(k)=\sigma_{a}^{2} \sum_{j=0}^{\infty} \psi_{j} \psi_{j+k}$.

2. $\operatorname{Var}\left(X_{t}\right)=\gamma_{x}(0)=\sigma_{a}^{2} \sum_{j=0}^{\infty} \psi_{j}^{2}<\infty$, pelas condições do Teorema 4.3.

3. $f_{x}(\lambda)=2 \sigma_{a}^{2} \Psi\left(e^{-2 \pi i \lambda}\right) \Psi\left(e^{2 \pi i \lambda}\right)=2 \sigma_{a}^{2}\left|\Psi\left(e^{-2 \pi i \lambda}\right)\right|^{2}, \quad 0 \leq \lambda \leq \pi$.

Por outro lado, o processo linear geral, $X_{t}=\Psi(B) a_{t}$, pode ser escrito como $\Psi^{-1}(B) X_{t}=a_{t}$, de modo que ao expandir $\Psi^{-1}(B)$ em potências de $B$ temos a representação auto-regressiva infinita de $\left\{X_{t}\right\}$ dada por 


$$
\begin{aligned}
\Psi^{-1}(B) X_{t} & =a_{t} \\
\Pi(B) X_{t} & =a_{t} \\
\left(1-\pi_{1} B-\pi_{2} B^{2}-\ldots\right) X_{t} & =a_{t} \\
X_{t} & =\pi_{1} X_{t-1}+\pi_{2} X_{t-2}+\ldots+a_{t} .
\end{aligned}
$$

A função $\Pi(B)=1-\sum_{j=1}^{\infty} \pi_{j} B^{j}$ denomina-se a função geradora dos pesos $\pi_{j}$.

Note que se multiplicarmos ambos os lados da equação $\Pi(B) X_{t}=a_{t}$ pela função $\Psi(B)$ obtemos a expressão $\Psi(B) \Pi(B) X_{t}=\Psi(B) a_{t}$, de modo que $\Pi(B)=$ $[\Psi(B)]^{-1}$ é a equação que relaciona os polinômios $\Pi(B)$ e $\Psi(B)$.

Finalmente note que o processo $\left\{X_{t}\right\}$ é estacionário se $\Psi(B)$ converge sobre ou dentro do círculo unitário e é invertível se $\Pi(B)$ converge sobre ou dentro do círculo unitário.

\subsubsection{Processos Auto-regressivos de Médias Móveis}

Como se especificou anteriormente a série temporal $\left\{X_{t}\right\}$ pode ser representada através de um dos processos lineares infinitos (4.5) e (4.6). Uma classe de modelos mais parcimoniosos, que fazem parte destes e que são muito usados na prática, são os modelos $\operatorname{ARMA}(p, q)$.

\section{Processos Auto-regressivos}

Se no modelo (4.6) $\pi_{j}=\phi_{j}$, para $j \leq p \quad$ e $\pi_{j}=0$, para $j \geq p+1$, ou seja, se

$$
X_{t}=\phi_{1} X_{t-1}+\phi_{2} X_{t-2}+\ldots+\phi_{p} X_{t-p}+a_{t}, \quad \phi_{p} \neq 0
$$

então $\left\{X_{t}\right\}$ é um processo auto-regressivo de ordem $\mathrm{p}$, denotado $\mathbf{A R}(\mathrm{p})$.

Podemos reescrever $X_{t}$ utilizando o operador $B$, isto é,

$$
\begin{aligned}
X_{t}=\phi_{1} X_{t-1}+\phi_{2} X_{t-2}+\ldots & +\phi_{p} X_{t-p}+a_{t} \\
\left(1-\phi_{1} B-\phi_{2} B^{2}-\ldots-\phi_{p} B^{p}\right) X_{t} & =a_{t} \\
\Phi_{p}(B) X_{t} & =a_{t}
\end{aligned}
$$




\section{Processos de Médias Móveis}

Se no modelo (4.5) os coeficientes $\psi_{j}=-\theta_{j}$, para $j \leq q \quad$ e $\quad \psi_{j}=0, \quad$ para $\quad j \geq$ $q+1$, ou seja, se

$$
X_{t}=a_{t}-\theta_{1} a_{t-1}-\theta_{2} a_{t-2}-\ldots-\theta_{q} a_{t-q}, \quad \theta_{q} \neq 0
$$

então $\left\{X_{t}\right\}$ é um processo de médias móveis de ordem q, denotado $\mathbf{M A}(q)$.

Também podemos reescrever $X_{t}$ através do operador $B$ na forma

$$
\begin{aligned}
& X_{t}=a_{t}-\theta_{1} a_{t-1}-\theta_{2} a_{t-2}-\ldots-\theta_{q} a_{t-q} \\
& X_{t}=\left(1-\theta_{1} B-\theta_{2} B^{2}-\ldots-\theta_{q} B^{q}\right) a_{t} \\
& X_{t}=\Theta_{q}(B) a_{t} .
\end{aligned}
$$

As principais propriedades dos processos $\operatorname{AR}(p)$ e $\operatorname{MA}(q)$ são:

1. O processo $\operatorname{AR}(p)$ é sempre invertível e é estacionário se as raízes de $\Phi_{p}(B)=$ 0 caírem fora do círculo unitário.

2. O processo $\operatorname{MA}(q)$ é sempre estacionário e é invertível se as raízes de $\Theta_{q}(B)=0$ caírem fora do círculo unitário.

3. A função de autocorrelação do processo $\mathrm{MA}(q)$ identifica o processo pois

$$
\gamma(k)= \begin{cases}\sigma_{a}^{2}\left(-\theta_{k}+\theta_{1} \theta_{k-1}+\ldots+\theta_{q-k} \theta_{q}\right) & k=1,2, \ldots, q \\ 0 & k>q .\end{cases}
$$

Em particular, a variância do processo é dada por

$$
\gamma(0)=\sigma_{a}^{2} \sum_{j=0}^{q} \theta_{j}^{2}
$$

e a função de autocorrelação por

$$
\rho(k)= \begin{cases}\frac{-\theta_{k}+\theta_{1} \theta_{k-1}+\ldots+\theta_{q-k} \theta_{q}}{1+\theta_{1}^{2}+\ldots+\theta_{q}^{2}} & k=1,2, \ldots, q . \\ 0 & k>q .\end{cases}
$$


4. No caso do processo $\operatorname{AR}(p)$ é a função de autocorrelação parcial quem identifica o processo e para conhecê-la explicitamente basta resolver as equações de Yule-Walker, dadas em (4.1), notando que

$$
\Phi_{k k}= \begin{cases}\phi_{k k} & k=1,2, \ldots, p \\ 0 & k>p\end{cases}
$$

5. A função de densidade espectral do processo $\operatorname{AR}(p)$ é

$$
f(\nu)=\frac{2 \sigma_{a}^{2}}{\left|1-\phi_{1} e^{-2 \pi i \nu}-\ldots-\phi_{p} e^{-2 \pi p i \nu}\right|^{2}}, \quad 0 \leq \nu \leq \frac{1}{2} .
$$

6. A função de densidade espectral do processo $\operatorname{MA}(q)$ é

$$
f(\nu)=2 \sigma_{a}^{2} \quad\left|1-\theta_{1} e^{-2 \pi i \nu}-\ldots-\theta_{q} e^{-2 \pi q i \nu}\right|^{2}, \quad 0 \leq \nu \leq \frac{1}{2} .
$$

Teorema 4.4 Sob a hipótese que o processo $\left\{X_{t}, t=1, \ldots, T\right\}$ segue um modelo auto-regressivo de ordem $p$, as autocorrelações parciais estimadas de ordem maior que p são assintoticamente independentes e distribuídas normalmente com variâncias

$$
\operatorname{Var}\left(\widehat{\phi}_{k k}\right) \simeq \frac{1}{T} \quad k \geq p+1
$$

Para maiores detalhes, consultar Jenkins(1956), Daniels(1956) e Brockwell-Davis (1991).

\section{Processos Auto-regressivos de Médias Móveis}

$O$ processo $\left\{X_{t}\right\}$ também pode ser representado através de uma mistura de um processo $\operatorname{AR}(p)$ e um processo $\operatorname{MA}(q)$ conhecido como o processo $\operatorname{ARMA}(p, q)$ e dado pela forma

$$
\begin{aligned}
X_{t}=\phi_{1} X_{t-1}+\ldots & +\phi_{p} X_{t-p}-\theta_{1} a_{t-1}-\ldots-\theta_{q} a_{t-q}+a_{t} \\
\left(1-\phi_{1} B-\ldots-\phi_{p} B^{p}\right) X_{t} & =\left(1-\theta_{1} B-\ldots-\theta_{q} B^{q}\right) a_{t} \quad \text { com } \quad \phi_{p} \neq 0, \quad \theta_{q} \neq 0 \\
\Phi_{p}(B) X_{t} & =\Theta_{q}(B) a_{t} .
\end{aligned}
$$

As principais propriedades dos processos $\operatorname{ARMA}(p, q)$ são:

1. O processo $\operatorname{ARMA}(p, q)$ é estacionário se as raízes do polinômio $\Phi_{p}(B)$ caírem fora do círculo unitário. 
2. O processo é invertível se as raízes do polinômio $\Theta_{q}(B)$ caírem fora do círculo unitário.

3. A função de autocovariância é dada por

$$
\begin{aligned}
\gamma(k)= & \phi_{1} \gamma(k-1)+\ldots+\phi_{p} \gamma(k-p)-\theta_{1} \gamma_{x a}(k-1)-\ldots- \\
& \theta_{q} \gamma_{x a}(k-q)+\gamma_{x a}(k)
\end{aligned}
$$

onde $\quad \gamma_{x a}(k-i)=E\left[X_{t-k} a_{t-i}\right]$.

Se $k=0$ temos a variância do processo dada por

$$
\gamma(0)=\phi_{1} \gamma(1)+\ldots+\phi_{p} \gamma(p)-\theta_{1} \gamma_{x a}(-1)-\ldots-\theta_{q} \gamma_{x a}(-q)+\sigma_{a}^{2}
$$

e para $k>q$ temos que

$$
\gamma(k)=\phi_{1} \gamma(k-1)+\ldots+\phi_{p} \gamma(k-p)
$$

e desta equação deriva-se a função de autocorrelação do processo,

$$
\rho(k)=\phi_{1} \rho(k-1)+\ldots+\phi_{p} \rho(k-p) \quad \text { quando } \quad k \geq q+1,
$$

observando-se que a fac quando $k \geq q+1$ satisfaz uma equação em diferenças homogênea de ordem $p$. Assim $\rho(k), k>q+1$ é uma mistura de funções exponenciais e/ou senóides e os valores $\rho(1), \ldots, \rho(q)$ dependem tanto dos parâmetros auto-regressivos quanto dos parâmetros de médias móveis e servem como os valores iniciais da equação em diferenças (4.8).

4. A função de autocorrelação parcial, por depender dos parâmetros da função de autocorrelação também é uma mistura de exponenciais e senóides.

5. A função de densidade espectral do processo $\operatorname{ARMA}(\mathrm{p}, \mathrm{q})$ é dada por

$$
f(\nu)=2 \sigma_{a}^{2} \frac{\left|\Theta_{q}\left(e^{-2 \pi i \nu}\right)\right|^{2}}{\left|\Phi_{p}\left(e^{-2 \pi i \nu}\right)\right|^{2}}=2 \sigma_{a}^{2} \frac{\left|1-\theta_{1} e^{-2 \pi i \nu}-\ldots-\theta_{q} e^{-2 \pi q i \nu}\right|^{2}}{\left|1-\phi_{1} e^{-2 \pi i \nu}-\ldots-\phi_{p} e^{-2 \pi p i \nu}\right|^{2}}, \quad 0 \leq \nu \leq \frac{1}{2} .
$$

\subsubsection{Processos Não Estacionários e Processos ARIMA}

Quando temos uma série temporal $\left\{X_{t}, t=0,1, \ldots, T-1\right\}$ linear e estacionária, ela pode ser representada através de um modelo $\operatorname{ARMA}(p, q)$. Caso a série não seja estacionária devemos primeiro aplicar alguma transformação adequada para torná-la estacionária. 
1. Quando a série não tem média constante e/ou apresenta uma tendência polinomial, a transformação que a torna estacionária é a diferenciação da série a qual se faz através do operador de diferenças $\nabla^{d}=(1-B)^{d}$ e é tal que

$$
\nabla^{d} X_{t}=(1-B)^{d} X_{t} .
$$

Quando $d=1, \quad \nabla X_{t}=(1-B) X_{t}=X_{t}-X_{t-1}$. Na prática, raramente $d>2$.

Em geral $\nabla^{d} X_{t}=(1-B)^{d} X_{t}$, é o operador da $d$-ésima diferença de $\left\{X_{t}\right\}$.

2. Quando a série não tem variância constante, uma transformação do tipo Box-Cox(1964) pode torná-la estacionária.

Para maiores detalhes ver Wei(1990).

Quando a não estacionariedade é do tipo descrito no item (1) dizemos que a série apresenta não estacionariedade homogênea. Neste caso o tipo de modelo estocástico que representa a série são os modelos $\operatorname{ARIMA}(p, d, q)$ dados por

$$
\varphi_{p+d}(B) X_{t}=\Theta_{q}(B) a_{t}
$$

$\operatorname{com} \varphi(B)$ um operador auto-regressivo não estacionário de ordem $p+d \operatorname{com} d$ das raízes de $\varphi(B)=0$ unitárias, sendo que as demais caem fora do círculo unitário e $\Theta(B)$ um operador de médias móveis de ordem $q$. Logo, (4.9) pode ser escrito como

$$
\Phi_{p}(B)(1-B)^{d} X_{t}=\Theta_{q}(B) a_{t}
$$

onde $\Phi_{p}(B)(1-B)^{d}=\varphi_{p+d}(B)$ com $\Phi_{p}(B)$ um operador auto-regressivo estacionário.

O modelo (4.9) também pode ser escrito na forma

$$
\Phi_{p}(B) W_{t}=\Theta_{q}(B) a_{t}
$$

com $W_{t}=\nabla^{d} X_{t}$ uma série estacionária. Assim a $d$-ésima diferença da série $X_{t}$ pode ser representada por um processo ARMA estacionário e invertível.

O processo $X_{t}$ pode ser recuperado somando (ou integrando) (4.11) $d$-vezes. Por esta razão (4.10) é denominado auto-regressivo integrado de médias móveis.

Existem três formas de representar o modelo $\operatorname{ARIMA}(p, d, q)$ : 


\section{Forma de Equação de Diferenças}

$$
X_{t}=\varphi_{1} X_{t-1}+\varphi_{2} X_{t-2}+\ldots+\varphi_{p+d} X_{t-p-d}-\theta_{1} a_{t-1}-\ldots-\theta_{q} a_{t-q}+a_{t}
$$

onde $X_{t}$ depende de seus $p-d$ valores passados e de $q$ valores passados e presente de $a_{t}$.

\section{Forma de Choques Aleatórios}

$$
X_{t}=a_{t}+\psi_{1} a_{t-1}+\psi_{2} a_{t-2}+\ldots=\Psi(B) a_{t}
$$

com

$$
\varphi_{p+d}(B) \Psi(B)=\Theta_{q}(B) .
$$

Assim, os coeficientes $\psi_{j}$ de (4.12) podem ser obtidos equacionando os coeficientes de $B$ na expansão de (4.13) dada por

$$
\left(1-\varphi_{1} B-\ldots-\varphi_{p+d} B^{p+d}\right)\left(1+\psi_{1} B+\psi_{2} B^{2}+\ldots\right)=1-\theta_{1} B-\ldots-\theta_{q} B^{q} .
$$

\section{Forma Invertida do Modelo}

$$
X_{t}=\pi_{1} X_{t-1}+\pi_{2} X_{t-2}+\ldots+a_{t} .
$$

Os coeficientes $\pi$ 's podem ser obtidos equacionando os coeficientes das potências de $B$ na expansão de $\varphi(B)=\Theta(B) \Pi(B)$ dada por

$$
\left(1-\varphi_{1} B-\ldots-\varphi_{p+d} B^{p+d}\right)=\left(1-\theta_{1} B-\ldots-\theta_{q} B^{q}\right)\left(1-\pi_{1} B-\pi_{2} B^{2}-\ldots\right) .
$$

\subsubsection{Processos Sazonais e Modelos SARIMA}

Quando uma série temporal tende a repetir seu comportamento em intervalos de tempo de cumprimento $\mathrm{s}$ diz-se que a série contém um componente sazonal de período s. Esta informação deve ser levada em conta na análise da série temporal em dois sentidos, primeiro verificando se a série $X_{t}, X_{t-s}, X_{t-2 s}, \ldots$ é estacionária e, segundo, incorporando a sazonalidade no modelo ARMA. 


\section{Diferenciação Sazonal}

Quando existe não estacionariedade nas componentes $X_{t}, X_{t-s}, X_{t-2 s}, \ldots$, da série, a transformação

$$
\nabla_{s}^{D}=\left(1-B^{s}\right)^{D}
$$

pode torná-la estacionária.

O efeito deste operador sobre a série $\left\{X_{t}\right\}$ é encontrar a $D$-ésima diferença entre observações separadas por $s$ etapas. Assim se $D=1$ temos a diferenciação sazonal de primeira ordem dada por

$$
Z_{t}=\nabla_{s} X_{t}=\left(1-B^{s}\right) X_{t}=X_{t}-B^{s} X_{t}=X_{t}-X_{t-s}
$$

onde $\left\{Z_{t}\right\}$ é possivelmente estacionária na sua componente sazonal.

\section{Modelo Multiplicativo}

Suponha que a série $\left\{X_{t}, t \in \mathbf{Z}\right\}$ de média zero é sazonal e é representada através do modelo ARIMA(p,d,q) dado por

$$
\Phi_{p}(B) \nabla^{d} X_{t}=\Theta_{q}(B) \alpha_{t},
$$

onde $\Phi_{p}(B)$ e $\Theta_{q}(B)$ são polinômios de ordem $p$ e $q$, satisfazendo as condições de estacionariedade e invertibilidade, respectivamente, e $\nabla^{d}=(1-B)^{d}$ é o operador de diferenças não-sazonais.

Já que em (4.14) não está incluída a componente sazonal, os resíduos $\alpha_{t}$ não são independentes, ou seja, a série $\left\{\alpha_{t}\right\}$ apresenta correlações significativas para variáveis separadas por $s$ etapas, de modo que o processo $\left\{\alpha_{t}\right\}$ pode ser representado pelo modelo $\operatorname{ARIMA}(P, D, Q)_{s}$

$$
\begin{aligned}
\Phi_{P}\left(B^{s}\right) \nabla_{s}^{D} \alpha_{t} & =\Theta_{Q}\left(B^{s}\right) a_{t} \\
\left(1-\Phi_{1} B^{s}-\ldots-\Phi_{P} B^{P s}\right)\left(1-B^{s}\right)^{D} \alpha_{t} & =\left(1-\Theta_{1} B^{s}-\ldots-\Theta_{Q} B^{Q s}\right) a_{t}
\end{aligned}
$$

onde a série $\left\{a_{t}\right\}$ é agora um ruído branco; $\Phi_{P}\left(B^{s}\right)$ e $\Theta_{Q}\left(B^{s}\right)$ são polinômios sazonais de ordem $P$ e $Q$, respectivamente, satisfazendo condições de estacionariedade e invertibilidade e $\nabla_{s}^{D}$ o operador de diferenças sazonais.

Isolando $\alpha_{t}$ em (4.14) e substituindo em (4.15) obtemos o modelo geral multiplicativo para séries sazonais

$$
\Phi_{P}\left(B^{s}\right) \Phi_{p}(B) \nabla^{d} \nabla_{s}^{D} X_{t}=\Theta_{Q}\left(B^{s}\right) \Theta_{q}(B) a_{t}
$$


conhecido como o modelo sazonal auto-regressivo integrado de médias móveis de ordem $(p, d, q)(P, D, Q)_{s}$ ou $\operatorname{SARIMA}(\mathbf{p}, \mathbf{d}, \mathbf{q})(\mathbf{P}, \mathbf{D}, \mathbf{Q})_{\mathbf{s}}$.

\subsection{Processos Multivariados}

\subsubsection{Modelos Lineares Vetoriais Gerais}

As representações (4.5) e (4.6) também são válidas para processos vetoriais (Wei 1990). Logo,

a) $\mathrm{O}$ processo $r$-vetorial $\left\{\mathrm{X}_{\mathrm{t}}\right\}$ de média zero pode ser escrito como uma combinação linear de uma seqüência de vetores ruído branco $r$-dimensionais da forma

$$
\begin{aligned}
\mathbf{X}(\mathbf{t}) & =\mu+\mathbf{a}_{\mathrm{t}}+\psi_{\mathbf{1}} \mathbf{a}_{\mathrm{t}-1}+\psi_{2} \mathbf{a}_{\mathrm{t}-2}+\ldots \\
& =\mu+\sum_{s=0}^{\infty} \psi_{\mathbf{s}} \mathbf{B}^{\mathbf{s}} \mathbf{a}_{\mathbf{t}}=\mu+\Psi(\mathbf{B}) \mathbf{a}_{\mathbf{t}}
\end{aligned}
$$

onde os $\psi_{\mathrm{s}}$ são matrizes $r \times r \operatorname{com} \psi_{\mathrm{o}}=\mathbf{I}_{\mathbf{r x r}}$ e os $\mathrm{a}_{\mathrm{t}}$ 's são vetores ruído branco $r$-dimensionais com média zero e estrutura de covariância

$$
E\left[\mathrm{a}_{\mathbf{t}} \mathrm{a}_{\mathbf{t}+\mathrm{k}}\right]=\left\{\begin{array}{lll}
\Sigma & \text { se } & k=0 \\
\mathbf{0} & \text { se } & k \neq 0
\end{array}\right.
$$

e $\Sigma$ é uma matrix simétrica de dimensão $r \times r$ e positiva definida.

O processo (4.16) é estacionário se os coeficientes das matrizes $\psi_{\mathbf{s}}$ satisfazem $\sum_{s=-\infty}^{\infty} \psi_{i j, s}^{2}<\infty$ para $i=1, \ldots, r$ e $j=1, \ldots, r$.

b) $O$ processo $r$-vetorial $\left\{\mathrm{X}_{\mathbf{t}}\right\}$ de média zero também pode ser representado numa forma auto-regressiva infinita mais um vetor de ruído branco, ou seja

$$
\begin{aligned}
\mathbf{X}(\mathbf{t}) & =\pi_{1} \mathbf{X}_{\mathbf{t}-1}+\pi_{2} \mathbf{X}_{\mathbf{t}-2}+\ldots+\mathbf{a}_{\mathbf{t}} \\
\Pi(\mathbf{B}) \mathbf{X}_{\mathbf{t}} & =\mathbf{a}_{\mathbf{t}}
\end{aligned}
$$

onde os $\pi_{\mathbf{s}}$ são matrizes $r \mathrm{x} r$ de coeficientes auto-regressivos.

O processo (4.17) é invertível se $\sum_{s=-\infty}^{\infty}\left|\pi_{i j, s}\right|<\infty$ para $i=1, \ldots, r \mathrm{e}$ $j=1, \ldots, r$.

Invertibilidade não necessariamente implica estacionariedade (e vice-versa). Para que um processo vetorial com representação auto-regressiva invertível seja 
estacionário é preciso que todas as raízes do determinante do polinômio autoregressivo matricial $\Pi(B)$ caiam fora do círculo unitário. Similarmente, para que um processo vetorial com representação de médias móveis estacionário seja invertível, todas as raízes do determinante do polinômio matricial $\Psi(B)$ devem cair fora do círculo unitário, ou seja, $|\Pi(B)| \neq 0$ e $|\Psi(B)| \neq 0$ para $|B| \leq 0$.

\subsubsection{Processos ARMA Vetoriais}

Como no caso univariado, existe uma classe de processos mais parcimoniosos ARMA vetoriais para representar o processo $r$-dimensional $\mathbf{X}(\mathbf{t})=\left[X_{1 t}, X_{2 t}, \ldots, X_{r t}\right]^{\prime}$ e são dados por

$$
\Phi_{\mathbf{p}}(\mathbf{B}) \mathbf{X}_{\mathbf{t}}=\Theta_{\mathrm{q}}(\mathbf{B}) \mathbf{a}_{\mathbf{t}}
$$

onde $\Phi_{p}(B)=\Phi_{0}-\Phi_{1} B-\ldots-\Phi_{p} B^{p}$ e $\Theta_{q}(B)=\Theta_{0}-\Theta_{1} B-\ldots-\Theta_{q} B^{q}$ são polinômios matriciais de ordem $p$ e $q$, respectivamente.

Quando $p=0$ temos o processo $\mathbf{M A}(\mathbf{q})$ vetorial

$$
X_{t}=a_{t}-\Theta_{1} a_{t-1}-\ldots-\Theta_{q} a_{t-q}, \quad \Theta_{q} \not \equiv 0 .
$$

Quando $q=0$ temos o processo $\mathbf{A R}(\mathbf{p})$ vetorial

$$
X_{t}=\Phi_{1} X_{t-1}-\ldots-\Phi_{p} X_{t-p}+a_{t}, \quad \Phi_{p} \not \equiv 0 .
$$

O processo (4.18) é estacionário se as raízes do determinante $\left|\Phi_{p}(B)\right|$, caírem fora do círculo unitário. Neste caso pode-se obter a representação (4.16)

$$
\mathbf{X}_{\mathrm{t}}=\Psi(\mathbf{B}) \mathbf{a}_{\mathrm{t}}
$$

onde

satisfazendo $\sum_{s=0}^{\infty}\left|\Psi_{s}\right|<\infty$.

$$
\Psi(\mathbf{B})=\left[\Phi_{\mathbf{p}}(\mathbf{B})\right]^{-1} \Theta_{\mathbf{q}}(\mathbf{B})=\sum_{\mathrm{s}=0}^{\infty} \Psi_{\mathbf{s}} \mathbf{B}^{\mathbf{s}}
$$

O processo (4.18) é invertível se as raízes do determinante $\left|\Theta_{q}(B)\right|$, caírem fora do círculo unitário. Neste caso pode-se obter a representação (4.17)

$$
\Pi(\mathbf{B}) \mathbf{X}_{\mathrm{t}}=\mathrm{a}_{\mathrm{t}}
$$

onde

$$
\Pi(\mathbf{B})=\left[\Theta_{\mathrm{q}}(\mathbf{B})\right]^{-1} \Phi_{\mathbf{p}}(\mathbf{B})=\mathbf{I}-\sum_{\mathrm{s}=1}^{\infty} \Pi_{\mathrm{s}} \mathbf{B}^{\mathbf{s}}
$$

tal que $\sum_{s=0}^{\infty}\left|\Pi_{s}\right|<\infty$ 


\subsubsection{Processos ARMA Vetoriais Não Estacionários}

Se o vetor $r$-dimensional $\left\{\mathbf{X}_{\mathbf{t}}, t=0, \pm 1, \pm 2, \ldots\right\}$ for não-estacionário homogêneo, então ele pode ser convertido em estacionário através da transformação $D(B) \mathbf{X}(\mathrm{t})$ onde $D(B)$ é um operador matricial de diferenças da forma

$$
D(B)=\left[\begin{array}{cccc}
(1-B)^{d_{1}} & \multicolumn{2}{c}{\underset{\sim}{ }} & \\
& (1-B)^{d_{2}} & & \\
\underset{\sim}{0} & & \ddots & \\
& & (1-B)^{d_{r}}
\end{array}\right]
$$

$\operatorname{com}\left(d_{1}, d_{2}, \ldots, d_{r}\right)$ um conjunto de inteiros não negativos.

Logo para o processo $\left\{\mathbf{X}_{\mathbf{t}}\right\}$ temos o modelo VARMA

$$
\Phi_{\mathbf{P}} \mathbf{D}(\mathbf{B}) \mathbf{X}_{\mathbf{t}}=\Theta_{\mathrm{Q}}(\mathbf{B}) \mathbf{a}_{\mathbf{t}}
$$

onde as raízes de $\left|\Phi_{p}(B)\right|=0$ e de $\left|\Theta_{q}(B)\right|=0$ caem dentro do círculo unitário.

Para maiores detalhes consultar Box et al.(1994), Wei(1990), Brockwell-Davis (1991). 


\section{Capítulo 5}

\section{Métodos de Comparação de Séries Temporais}

Considere duas séries $r$-variadas, finitas e estacionárias

$$
\left\{\mathbf{X}^{(1)}(t) ; t=1, \ldots, T\right\} \quad \text { e } \quad\left\{\mathbf{X}^{(2)}(t) ; t=1, \ldots, T\right\}
$$

com $\mathbf{X}^{(i)}(t)=\left[X_{1}^{(i)}(t), \ldots, X_{r}^{(i)}(t)\right]^{\prime}$ um vetor que contém $r$ séries estacionárias, $i=1,2$.

Nosso interesse é saber se as duas séries multivariadas foram geradas por um mesmo processo estacionário $\{\mathbf{X}(t)\}$.

$\mathrm{O}$ caso mais simples acontece quando $r=1$ e as duas séries são independentes, ou seja, temos duas séries univariadas independentes $\left\{\mathbf{X}^{(1)}(t)\right\}=\left\{X_{1 t}\right\}$ e $\left\{\mathbf{X}^{(2)}(t)\right\}=\left\{X_{2 t}\right\}$ e queremos saber se elas são geradas pelo mesmo processo univariado $\{X(t)\}$. Para este caso, apresentam-se 4 testes descritos na próxima seção, dois deles no domínio das freqüências e os outros dois no domínio do tempo.

\subsection{Comparação de Duas Séries Univariadas}

Assumindo que as duas séries univariadas $\left\{X_{i t}\right\}, i=1,2$ são Gaussianas com $f_{i}(\lambda), \gamma_{i}(j)$ e $\rho_{i}(j), i=1,2$, suas funções de densidade espectral, autocovariância e autocorrelação, respectivamente, o problema se reduz a testar uma das seguintes 
hipóteses:

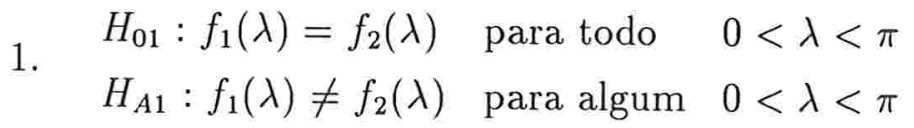

2. $H_{02}: \gamma_{1}(j)=\gamma_{2}(j) \quad$ para todo $j=0, \pm 1, \ldots$

$H_{A 2}: \gamma_{1}(j) \neq \gamma_{2}(j)$ para algum $j=0, \pm 1, \ldots$

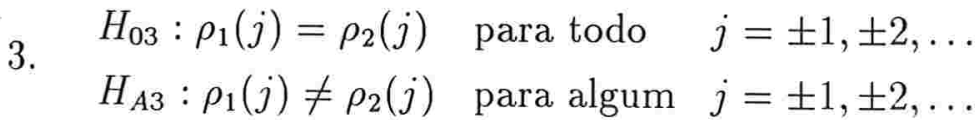

Baseados nos resultados do Capítulo 3, Coates e Diggle(1986) desenvolveram os dois testes seguintes no domínio das freqüências para testar a hipótese (5.1).

\subsubsection{Teste das Somas Acumuladas (SA-Coates e Diggle)}

Seja $I_{i}^{(T)}(\lambda)$ o periodograma da série $\left\{X_{i t}\right\}, i=1,2$. Sob as condições do Teorema 3.6 temos que, assintoticamente, $I_{i}^{(T)}(\lambda) \sim f_{i}(\lambda) \chi_{2}^{2} / 2, \lambda \not \equiv 0, \pi$. Definindo as razões espectrais

$$
J(\lambda)=\frac{I_{1}^{(T)}(\lambda)}{I_{2}^{(T)}(\lambda)} \quad \text { e } \quad U(\lambda)=\frac{f_{1}(\lambda)}{f_{2}(\lambda)}, \quad 0<\lambda<\pi
$$

com $\left\{X_{1 t}\right\}$ e $\left\{X_{2 t}\right\}$ independentes temos que, assintoticamente,

$$
J(\lambda)=\frac{I_{1}^{(T)}(\lambda)}{I_{2}^{(T)}(\lambda)} \sim \frac{f_{1}(\lambda) \chi_{2}^{2} / 2}{f_{2}(\lambda) \chi_{2}^{2} / 2} \sim U(\lambda) F_{2,2},
$$

onde $F$ é a distribuição de Fisher-Snedecor.

Demostra-se que

$$
\ln J(\lambda) \sim \log \text { ística }\{\ln U(\lambda), 1\}
$$

onde logística $(a, 1)$ denota a distribuição logística com função de distribuição dada por

$$
F(x)=\left\{1+e^{-(x-a)}\right\}^{-1}, \quad-\infty<x<\infty .
$$


Lembre que se $X \sim \operatorname{logística}(a, 1)$ então

$$
E(X)=a \quad \text { e } \quad \operatorname{Var}(X)=\pi^{2} / 3 .
$$

Logo $\ln J(\lambda)$ é um estimador não viesado, embora inconsistente, para $\ln U(\lambda)$ e sua variância assintótica independe de $\lambda$.

Também, como assintóticamente

$$
J(\lambda) \sim U(\lambda) F_{2,2}
$$

pode-se demostrar que os valores

$$
z_{i}=\ln \left(1+J^{-1}\left(\lambda_{i}\right)\right) \sim U\left(\lambda_{i}\right) \exp (1)
$$

$\operatorname{com} \lambda_{i}=\frac{2 \pi i}{T}, \quad i=1, \ldots, m$, onde $m=\left[\frac{T-1}{2}\right]$ e onde $\exp (1)$ denota a distribuição exponencial de média 1.

Sob a hipótese $H_{01}$, dada por (5.1), $U\left(\lambda_{i}\right)=1$ e, assintóticamente,

$$
z_{i} \sim \exp (1)
$$

Assim $c_{j}=\sum_{i=1}^{j} z_{i}$ constituem os pontos de um processo de Poisson e, conseqüentemente, $\left\langle\frac{c_{j}}{c_{m}}\right\rangle, j=1, \ldots, m$, é o vetor das estatísticas de ordem da distribuição uniforme no intervalo $(0,1)$.

O primeiro teste proposto por Coates e Diggle(1986), consiste em construir as estatísticas $\left\langle\frac{c_{j}}{c_{m}}\right\rangle, j=1, \ldots, m$ e usar a estatística de Kolmogorov-Smirnov (Apêndice C) para testar afastamentos da distribuição $U(0,1)$, ou equivalentemente, pode-se calcular simplesmente as estatísticas $z_{i}$ e aplicar o teste de Kolmogorov-Smirnov para testar afastamentos da distribuição exponencial de média 1.

Mais especificamente, o procedimento é o seguinte:

1. Obtenha os periodogramas $I_{1}^{(T)}\left(\lambda_{i}\right)$ de $\left\{X_{1 t}\right\}$ e $I_{2}^{(T)}\left(\lambda_{i}\right)$ de $\left\{X_{2 t}\right\}, \quad i=$ $1, \ldots, m$.

2. Encontre a razão dos periodogramas, $J\left(\lambda_{i}\right)=\frac{I_{1}^{(T)}\left(\lambda_{i}\right)}{I_{2}^{(T)}\left(\lambda_{i}\right)}, \quad i=1, \ldots, m$.

3. Calcule as estatísticas $z_{i}=\ln \left(1+J^{-1}\left(\lambda_{i}\right)\right), \quad i=1, \ldots, m$.

4. Calcule as somas acumuladas $c_{j}=\sum_{i=1}^{j} z_{i}, \quad j=1, \ldots, m$. 
5. Calcule as estatísticas $o_{j}=\frac{c_{j}}{c_{m}}, \quad j=1, \ldots, m$.

6. Aplique un teste de Kolmogorov-Smirnov para comparar a distribuição dos $o_{j}$ com a distribuição $U(0,1)$, e rejeite a hipótese $H_{01}$ dada por (5.1), ao nível de significância $\alpha$, se o nível descritivo do teste é menor que $\alpha$.

Uma outra alternativa para testar (5.1) e que exclui os passos 4,5 e 6, consiste em aplicar un teste de Kolmogorov-Smirnov para comparar a distribuição dos $z_{i}$ com a distribuição da exponencial de média 1 e rejeitar $H_{01}$, ao nível de significância $\alpha$, se o nível descritivo do teste é menor que $\alpha$.

Este teste apresenta a desvantagem de poder variar segundo a razão dos periodogramas $J$ utilizada, isto é, $J\left(\lambda_{i}\right)=\frac{I_{1}^{(T)}\left(\lambda_{i}\right)}{I_{2}^{(T)}\left(\lambda_{i}\right)}$ ou $J\left(\lambda_{i}\right)=\frac{I_{2}^{(T)}\left(\lambda_{i}\right)}{I_{1}^{(T)}\left(\lambda_{i}\right)}, \quad i=1, \ldots, m$.

\subsubsection{Teste da Razão de Verossimilhança (RV-Coates e Diggle)}

Especificando uma forma funcional para $\ln J(\lambda)$, pode-se construir um teste da razão de verossimilhança generalizada, dentro da estrutura da distribuição logística, de acordo com (5.4).

Adotando um modelo quadrático para $\ln U(\lambda)$

$$
\ln U(\lambda)=\alpha+\beta \lambda+\gamma \lambda^{2}
$$

temos que,

$$
H_{01}: f_{1}(\lambda)=f_{2}(\lambda), \quad 0<\lambda<\pi \quad \Longleftrightarrow \quad H_{01}: \alpha=\beta=\gamma=0 .
$$

Seja $t_{i}=\ln J\left(\lambda_{i}\right), i=1, \ldots, m$, então de (5.4) e (5.6) temos que, assintóticamente,

$$
t_{i} \sim \operatorname{logística}\left(\ln U\left(\lambda_{i}\right), 1\right)
$$

logo, a função de densidade de $t_{i}, i=1, \ldots, m$ é dada por

$$
f_{T}\left(t_{i}\right)=\frac{e^{-\left(t_{i}-\ln U\left(\lambda_{i}\right)\right)}}{\left\{1+e^{-\left(t_{i}-\ln U\left(\lambda_{i}\right)\right)}\right\}^{2}} \quad i=1, \ldots, m .
$$

Conseqüentemente, a função de verossimilhança dos $t_{i}$ é dada por 


$$
\begin{aligned}
L(t / \Theta)=\prod_{i=1}^{m} f_{T}\left(t_{i}\right) & =\frac{e^{\sum_{i=1}^{m}\left(-t_{i}+\ln U\left(\lambda_{i}\right)\right)}}{\prod_{i=1}^{m}\left\{1+e^{-\left(t_{i}-\ln U\left(\lambda_{i}\right)\right)}\right\}^{2}} \\
& =\frac{e^{\sum_{i=1}^{m}\left(-t_{i}+\alpha+\beta \lambda_{i}+\gamma \lambda_{i}^{2}\right)}}{\prod_{i=1}^{m}\left\{1+e^{\left(-t_{i}+\alpha+\beta \lambda_{i}+\gamma \lambda_{i}^{2}\right)}\right\}^{2}}
\end{aligned}
$$

$\mathrm{e}$

$$
\begin{aligned}
\mathrm{l}=\ln L & =\sum_{i=1}^{m}\left(-t_{i}+\ln U\left(\lambda_{i}\right)\right)-2 \sum_{i=1}^{m} \ln \left(1+e^{-t_{i}+\ln U\left(\lambda_{i}\right)}\right) \\
& =\sum_{i=1}^{m}\left(-t_{i}+\alpha+\beta \lambda_{i}+\gamma \lambda_{i}^{2}\right)-2 \sum_{i=1}^{m} \ln \left(1+e^{\left(-t_{i}+\alpha+\beta \lambda_{i}+\gamma \lambda_{i}^{2}\right)}\right) .
\end{aligned}
$$

Os estimadores de máxima verossimilhança de $\alpha, \beta$ e $\gamma$ podem ser obtidos numericamente através do algoritmo de Newton-Raphson e, para efeitos de uma convergência mais rápida, sugere-se usar as estimativas de mínimos quadrados ordinários ou ponderados como valores iniciais.

O segundo teste proposto por Coates e Diggle(1986) para testar a hipótese $H_{01}$ dada por (5.1), baseia-se na distribuição assintótica da razão de verossimilhança, ou seja, baseia-se no fato que

$$
R_{v}^{\prime}=-2 \ln \frac{\sup _{\Omega_{01}} L(\Theta / t)}{\sup _{\Omega} L(\Theta / t)} \sim \chi_{3}^{2} .
$$

Logo rejeita-se $H_{01}$ para valores grandes de $R_{v}^{\prime}$. Mais especificamente, os passos são descritos a seguir:

1. Obtenha os periodogramas $I_{1}^{(T)}\left(\lambda_{i}\right)$ de $\left\{X_{1 t}\right\}$ e $I_{2}^{(T)}\left(\lambda_{i}\right)$ de $\left\{X_{2 t}\right\}$.

2. Encontre a razão dos periodogramas, $J\left(\lambda_{i}\right)=\frac{I_{1}^{(T)}\left(\lambda_{i}\right)}{I_{2}^{(T)}\left(\lambda_{i}\right)}, \quad i=1, \ldots, m$.

3. Calcule as estatísticas $t_{i}=\ln J\left(\lambda_{i}\right), \quad i=1, \ldots, m$.

4. Estime os parâmetros $\alpha, \beta$ e $\gamma$ do modelo logístico quadrático, por máxima verossimilhança, usando o algoritmo de Newton-Raphson e colocando como valores iniciais as estimativas de mínimos quadrados.

5. Calcule a estatística da razão de verossimilhança, $R_{v}^{\prime}$. 
6. Rejeite $H_{01}$ ao nível de significância $\alpha$ se $R_{v}^{\prime}>C_{\alpha}$, onde $C_{\alpha}$ é tal que $P\left(\chi_{3}^{2}>C_{\alpha}\right)=\alpha$.

Os dois testes anteriores requerem que as duas séries tenham o mesmo tamanho.

Os dois testes seguintes, no domínio do tempo, foram propostos por Mélard e Roy(1984) e por Quenouille(1958) para testar as hipóteses $H_{02}$ e $H_{03}$ dadas por (5.2) e (5.3), respectivamente. Estes dois testes têm a vantagem de não requerer que as séries tenham o mesmo tamanho. Nestes testes, para simplificar a notação, utilizaremos $n_{1}$ e $n_{2}$ para indicar os tamanhos das amostras.

\subsubsection{Teste de Igualdade das Funções de Autocovariância (Mélard e Roy)}

Para testar a hipótese $H_{02}$ dada por (5.2), Mélard e Roy(1984) propuseram o teste descrito a seguir.

Seja $\left\{X_{k t}, t=1, \ldots, n_{k}\right\}$ séries estacionárias com momentos de ordem 1 e 2 dados por

$$
\begin{aligned}
\mu_{k} & =E\left[X_{k t}\right] \\
\gamma_{k}(j) & =E\left[\left(X_{k t}-\mu_{k}\right)\left(X_{k, t+j}-\mu_{k}\right)\right]
\end{aligned}
$$

para $k=1,2$ e onde todos os cumulantes de ordem 4 são nulos e tal que $\sum_{j=-\infty}^{\infty} \gamma_{k}^{2}(j)<\infty$. A normalidade do processo não é requerida, no entanto, a hipótese sobre os cumulantes indica que a distribuição do processo não está muito longe da normal.

Nesta seção nos referiremos a $\widehat{\gamma}_{k}$ por $c_{k}$ para simplificar a notação.

Considere então, para a $k$-ésima série o vetor $\mathbf{c}_{k}$ das primeiras $J+1$ autocovariâncias estimadas

$$
\mathrm{c}_{k}=\left[c_{k}(0), c_{k}(1), c_{k}(2), \ldots, c_{k}(J)\right]^{\prime}
$$

onde para cada $j=0,1, \ldots, J$ o estimador $c_{k}(j)$ é dado por

$$
c_{k}(-j)=c_{k}(j)=\frac{1}{n_{k}} \sum_{t=1}^{n_{k}-j}\left(X_{k t}-\overline{X_{k}}\right)\left(X_{k, t+j}-\overline{X_{k}}\right)
$$


e $\overline{X_{k}}=\frac{1}{n_{k}} \sum_{t=1}^{n_{k}} X_{k t}$ é a média amostral da $k$-ésima série.

A matriz de variâncias e covariâncias do vetor $\mathbf{c}_{k}$ é dada por

$$
\Sigma_{k}=\lim _{n_{k} \rightarrow \infty} n_{k} E\left[\left(\mathbf{c}_{k}-\gamma_{k}\right)\left(\mathbf{c}_{k}-\gamma_{k}\right)^{\prime}\right]
$$

como no Teorema 4.1. Daqui em diante supomos que $\Sigma_{k}$ é uma matriz positiva definida.

Temos, também, que assintoticamente

$$
\mathrm{c}_{k} \sim \mathrm{N}_{J+1}\left(\gamma_{k}, n_{k}^{-1} \Sigma_{k}\right)
$$

Sob a suposição que as duas realizações são independentes, $\mathbf{c}_{1}$ e $\mathbf{c}_{2}$ são dois vetores aleatórios independentes. Logo, assintoticamente,

$$
\mathbf{c}_{1}-\mathbf{c}_{2} \sim \mathrm{N}_{J+1}\left(\gamma_{1}-\gamma_{2}, n_{1}^{-1} \Sigma_{1}+n_{2}^{-1} \Sigma_{2}\right)
$$

Deseja-se testar a hipótese dada por (5.2). Sob esta hipótese, $\Sigma_{1}=\Sigma_{2}=\Sigma$ e

$$
\mathbf{c}_{1}-\mathbf{c}_{2} \sim \mathrm{N}_{J+1}\left(\mathbf{0},\left(n_{1}+n_{2}\right)\left(n_{1} n_{2}\right)^{-1} \Sigma\right)
$$

Colocando $n=n_{1}$ e supondo que $\frac{n_{1}}{n_{2}}$ é constante para $n \rightarrow \infty$ e, além disso, denotando

$$
\mathbf{Z}^{(n)}=\left(\frac{n_{1} n_{2}}{n_{1}+n_{2}}\right)^{1 / 2}\left(\mathbf{c}_{1}-\mathbf{c}_{2}\right)
$$

então,

$$
\mathbf{Z}^{(n)} \stackrel{\mathrm{D}}{\longrightarrow} \mathbf{Z} \quad \text { e } \quad \mathbf{Z}^{(n)^{\prime}} \Sigma^{-1} \mathbf{Z}^{(n)} \stackrel{\mathrm{D}}{\longrightarrow} \mathbf{Z}^{\prime} \Sigma^{-1} \mathbf{Z}
$$

onde $\mathbf{Z}$ representa a distribuição $N_{J+1}(0, \Sigma)$ e, portanto, $\mathbf{Z}^{\prime} \Sigma^{-1} \mathbf{Z}$ segue uma distribuição $\chi_{J+1}^{2}$.

A forma quadrática $\mathbf{Z}^{(n)^{\prime}} \Sigma^{-1} \mathbf{Z}^{(n)}$ é uma estatística similar àquela utilizada para efetuar testes sobre o vetor médio de uma população multivariada.

Se $\widehat{\Sigma}_{k}$ for um estimador convergente em probabilidade para $\Sigma_{k}$ teremos

$$
\widehat{\Sigma}^{(n)}=\left(n_{1}+n_{2}\right)^{-1}\left(n_{1} \widehat{\Sigma}_{1}+n_{2} \widehat{\Sigma}_{2}\right)
$$

um estimador convergente para $\Sigma \mathrm{e}\left(\widehat{\Sigma}^{(n)}\right)^{-1}$, um estimador convergente para $\Sigma^{-1}$. Assim, 


$$
\mathbf{Z}^{(n)} \stackrel{\mathrm{D}}{\longrightarrow} \mathbf{Z} \quad \text { e } \quad\left(\widehat{\Sigma}^{(n)}\right)^{-1} \stackrel{\mathrm{p}}{\longrightarrow} \Sigma^{-1}
$$

e pelo Teorema 4.4 de Billingsley(1968) tem-se que

$$
\left(\mathbf{Z}^{(n)},\left(\Sigma^{(n)}\right)^{-1}\right) \stackrel{\mathrm{D}}{\longrightarrow}\left(\mathrm{Z}, \Sigma^{-1}\right) .
$$

Definindo a função

$$
\mathrm{Q}=\mathrm{Q}\left(\mathrm{Z}, \Sigma^{-1}\right)=\mathbf{Z}^{\prime} \Sigma^{-1} \mathbf{Z}
$$

que é contínua em cada um dos elementos de $\mathbf{Z}$ e de $\Sigma^{-1}$, pelo Teorema 5.1 de Billingsley(1968), deriva-se que

$$
\mathrm{Q}^{(n)}=\mathrm{Q}\left(\mathbf{Z}^{(n)},\left(\Sigma^{(n)}\right)^{-1}\right) \stackrel{\mathrm{D}}{\longrightarrow} \mathrm{Q}\left(\mathrm{Z}, \Sigma^{-1}\right)
$$

e, como já tínhamos demonstrado que $\mathrm{Q}$ segue uma distribuição $\chi_{J+1}^{2}$, então

$$
\mathrm{Q}^{(n)} \stackrel{\mathrm{D}}{\longrightarrow} \chi_{J+1}^{2}
$$

A proposta de Mélard e Roy(1984) consiste em testar a hipótese $H_{02}$ dada por (5.2) utilizando a estatística $\mathrm{Q}^{(n)}$.

O procedimento técnico consiste dos seguintes passos:

1. Estime as funções de autocovariância $\gamma_{k}, k=1,2$, das séries $\left\{X_{1 t}\right\}$ e $\left\{X_{2 t}\right\}$ por meio de $\mathrm{c}_{k}, k=1,2$.

2. Para cada vetor de autocovariâncias do passo anterior, estime sua matriz de variâncias e covariâncias, $\Sigma_{k}$, usando $\hat{\theta}_{i}$ como estimador de $\theta_{i}$. $\widehat{\theta}_{i}$ e $\theta_{i}$ são definidos a seguir.

3. Encontre a matriz $\widehat{\Sigma}^{(\mathbf{n})}=\left(n_{1}+n_{2}\right)^{-1}\left(n_{1} \widehat{\Sigma}_{1}+n_{2} \widehat{\Sigma}_{2}\right)$.

4. Calcule a variável $\mathbf{Z}^{(\mathbf{n})}=\left(\frac{n_{1} n_{2}}{n_{1}+n_{2}}\right)^{1 / 2}\left(\mathbf{c}_{1}-\mathbf{c}_{2}\right)$, onde os vetores $\mathbf{c}_{k}$ são de dimensão $J+1$.

5. Calcule a estatística $\mathbf{Q}^{(\mathbf{n})}=\mathbf{Z}^{(\mathbf{n})^{\prime}}\left(\widehat{\Sigma}^{(\mathbf{n})}\right)^{-1} \mathbf{Z}^{(\mathbf{n})}$. Rejeite $H_{02}$, ao nível de significância $\alpha$, se $\mathrm{Q}^{(\mathrm{n})}>C_{\alpha}$, onde $C_{\alpha}$ é tal que $P\left(\chi_{J+1}^{2}>C_{\alpha}\right)=\alpha$. 
Tratamos agora da obtenção de um estimador convergente $\widehat{\Sigma}$ para $\Sigma$, onde

$$
\Sigma=\lim _{n \rightarrow \infty} n E\left[(\mathbf{c}-\gamma)(\mathbf{c}-\gamma)^{\prime}\right]
$$

De acordo com Anderson (1971) podemos escrever o elemento $(l, i), \quad l, i=$ $0,1, \ldots, J$, de $\Sigma$ na forma

$$
\begin{aligned}
\sigma_{l i} & =\theta_{i-l}+\theta_{i+l} \\
\operatorname{com} \quad \theta_{i} & =\sum_{j=-\infty}^{\infty} \gamma(j) \gamma(i+j) .
\end{aligned}
$$

Robinson(1977) propôs o seguinte estimador de $\theta_{i}$

$$
\widehat{\theta}_{i}=\sum_{j=-n+1}^{n-i-1} w\left(j b_{n}\right) c(j) c(i+j) .
$$

$\operatorname{com} w(\cdot)$ uma função absolutamente integrável, contínua na origem, no máximo com um número finito de descontinuidades e $|w(0)|=1$. Além disso, $b_{n}$ é uma seqüência que converge para zero e tal que $n b_{n} \rightarrow \infty$ quando $n \rightarrow \infty$, implicando que $E\left|\widehat{\theta}_{i}-\theta_{i}\right| \rightarrow 0$.

No entanto, não se pode assegurar que a matriz $\widehat{\Sigma}=\left(\widehat{\sigma}_{l i}\right), \quad l, i=0,1, \ldots, J$ com $\widehat{\sigma}_{l i}=\widehat{\theta}_{i-l}+\widehat{\theta}_{i+l}$ seja positiva definida.

Mélard e Roy(1984) propõem um novo estimador para $\theta_{i}$ da forma

$$
\tilde{\theta}_{i}=\sum_{j=-n+1}^{n-i-1} w\left(j b_{n}\right) w\left((i+j) b_{n}\right) c(j) c(i+j)
$$

com as mesmas hipóteses que Robinson(1977) exceto que $w^{2}(\cdot)$ é uma função integrável e a função $w_{j}=w\left(j b_{n}\right), j$ inteiro, uma função positiva. Loève(1978) mostra que a função $w_{j} c(j)$ é também uma função de autocovariância de forma que ao substituir $\gamma(j)$ por $w_{j} c(j)$ em (5.8) obtém-se uma matriz $\widehat{\Sigma}$ positiva definida como era requerida.

Finalmente pode-se demonstrar que $\tilde{\theta}_{i}$ converge para $\theta_{i}$ na norma $L_{1}$.

Para maiores detalhes consultar Mélard e Roy(1984). Neste artigo, a função $w$ utilizada é a janela de Bartlett dada por

$$
w_{j}\left(b_{n, H}\right)=\left\{\begin{array}{cl}
1-\frac{|j|}{b_{n, H}} & |j| \leq b_{n, H} \\
0 & |j|>b_{n, H} .
\end{array}\right.
$$




\subsubsection{Teste de Igualdade das Funções de Autocorrelação (Quenouille)}

Baseado nas suposições:

1. Se o ajuste de um modelo auto-regressivo para uma série $\left\{X_{t}\right\}$ for adequado, de modo que os resíduos sejam independentes, as autocorrelações parciais $v_{j} \mathrm{j}=0,1, \ldots$, destes resíduos são assintoticamente independentes e distribuídas normalmente com variâncias assintóticas $\frac{1}{n-j}$, onde $n$ é o tamanho da série;

2. O resultado anterior não é sensível a imprecisões no ajuste,

Quenouille(1958) propôs um teste para a hipótese $H_{03}$ dada por (5.3).

O procedimento de Quenouille para testar diferenças entre dois conjuntos de autocorrelações é dado pelos seguintes passos.

1. Obter a função de autocorrelação $\widehat{\rho}_{1}(j)$ e $\widehat{\rho}_{2}(j), \quad j=0,1, \ldots, J$ das séries $\left\{X_{1 t}\right\}$ e $\left\{X_{2 t}\right\}$, respectivamente.

2. Obter a média ponderada de $\widehat{\rho}_{1}(j)$ e $\widehat{\rho}_{2}(j)$, da forma $\widehat{\rho}(j)=\frac{n_{1} \widehat{\rho}_{1}(j)+n_{2} \widehat{\rho}_{2}(j)}{n_{1}+n_{2}}$, obtendo uma função de autocorrelação comum as duas séries; $n_{1}$ e $n_{2}$ são os tamanhos das séries $\left\{X_{1 t}\right\}$ e $\left\{X_{2 t}\right\}$, respectivamente.

3. Calcular a função de autocorrelação parcial estimada comum, $\widehat{\Phi}(k)$, a partir de $\hat{\rho}(j)$.

4. Identificar a ordem auto-regressiva $p$ através de $\widehat{\Phi}(k)$.

5. Estimar os $p$ coeficientes do modelo $\operatorname{AR}(p)$, resolvendo as equações de YuleWalker.

6. Filtrar cada série com estes coeficientes, isto é, ajustar a cada série o modelo $\operatorname{AR}(p)$ com os coeficientes comuns encontrados no item 5 e obter as duas séries residuais $\left\{\hat{a}_{1 t}\right\}$ e $\left\{\hat{a}_{2 t}\right\}$.

7. Calcular as facp, $v_{j}$ e $v_{j}^{\prime}$ das duas séries residuais $\left\{\hat{a}_{1 t}\right\}$ e $\left\{\hat{a}_{2 t}\right\}$, respectivamente. 
8. Testar se $\frac{v_{j}-v_{j}^{\prime}}{\sqrt{\frac{1}{n_{1}-j}+\frac{1}{n_{2}-\jmath}}}$ tem distribuição aproximadamente $N(0,1)$, ou equivalentemente, testar se

$$
S Q=\sum_{j=1}^{J} \frac{\left(v_{j}-v_{j}^{\prime}\right)^{2}}{\frac{1}{n_{1}-j}+\frac{1}{n_{2}-j}} \sim \chi_{J}^{2} .
$$

A hipótese $H_{03}$ dada por (5.3) é rejeitada, a um nível de significância $\alpha$, se $S Q>C_{\alpha}$, onde $C_{\alpha}$ é tal que $P\left(\chi_{J}^{2}>C_{\alpha}\right)=\alpha$.

\subsection{Comparação de Duas Séries Multivariadas}

Consideremos novamente as duas séries r-variadas,

$$
\begin{aligned}
& \left\{\mathbf{X}^{(1)}(t)=\left[X_{1}^{(1)}(t), X_{2}^{(1)}(t), \ldots, X_{r}^{(1)}(t)\right], \quad t \in \mathbf{Z}\right\} \\
& \left\{\mathbf{X}^{(2)}(t)=\left[X_{1}^{(2)}(t), X_{2}^{(2)}(t), \ldots, X_{r}^{(2)}(t)\right], \quad t \in \mathbf{Z}\right\} .
\end{aligned}
$$

Assume-se que as duas séries são observações de dois processos $r$-dimensionais $\left\{\mathbf{X}^{(1)}(t), t \in \mathbf{Z}\right\}$ e $\left\{\mathbf{X}^{(2)}(t), t \in \mathbf{Z}\right\}$, que podem ser vistos como marginais de um processo $2 r$-dimensional $\{\mathbf{X}(t), t \in \mathbf{Z}\}$. Assume-se, também, que este processo é estacionário pelo menos no sentido fraco e que sua função de covariância é absolutamente integrável. Isto garante a existência da função matricial de densidade espectral $\mathbf{f}(\lambda)$ dada por

$$
\mathbf{f}(\lambda)=\left[\begin{array}{ccc}
\mathbf{f}_{1,1}(\lambda) & \vdots & \mathbf{f}_{1,2}(\lambda) \\
\ldots \ldots & \ldots \ldots \ldots \\
\mathbf{f}_{2,1}(\lambda) & \vdots & \mathbf{f}_{2,2}(\lambda)
\end{array}\right]_{(2 \mathrm{r}) \times(2 \mathbf{r})}
$$

onde cada matriz $\left[\mathrm{f}_{i, j}\right]_{\mathrm{rxr}} \mathrm{i}, \mathrm{j}=1,2$ é a matriz de densidades espectrais e densidades espectrais cruzadas. Mais explicitamente,

$$
\mathbf{f}_{1,1}(\lambda)=\left[\begin{array}{cccc}
f_{11}^{(1)} & f_{12}^{(1)} & \ldots & f_{1 r}^{(1)} \\
f_{21}^{(1)} & f_{22}^{(1)} & \ldots & f_{2 r}^{(1)} \\
\vdots & \vdots & \ddots & \vdots \\
f_{r 1}^{(1)} & f_{r 2}^{(1)} & \vdots & f_{r r}^{(1)}
\end{array}\right] \quad \text { e } \quad \mathbf{f}_{2,2}(\lambda)=\left[\begin{array}{cccc}
f_{11}^{(2)} & f_{12}^{(2)} & \ldots & f_{1 r}^{(2)} \\
f_{21}^{(2)} & f_{22}^{(2)} & \ldots & f_{2 r}^{(2)} \\
\vdots & \vdots & \ddots & \vdots \\
f_{r 1}^{(2)} & f_{r 2}^{(2)} & \vdots & f_{r r}^{(2)}
\end{array}\right]
$$


são as matrizes de densidades espectrais na freqüência $\lambda$ de cada vetor $\mathrm{r}$-dimensional $\left\{\mathbf{X}^{(1)}(t)\right\}$ e $\left\{\mathbf{X}^{(2)}(t)\right\}$, respectivamente, e

$$
\mathrm{f}_{1,2}(\lambda)=\left[\begin{array}{cccc}
f_{11}^{(1.2)} & f_{12}^{(1.2)} & \ldots & f_{1 r}^{(1.2)} \\
f_{21}^{(1.2)} & f_{22}^{(1.2)} & \ldots & f_{2 r}^{(1.2)} \\
\vdots & \vdots & \ddots & \vdots \\
f_{r 1}^{(1.2)} & f_{r 2}^{(1.2)} & \vdots & f_{r r}^{(1.2)}
\end{array}\right] \quad \text { e } \quad \mathrm{f}_{2,1}(\lambda)=\left[\begin{array}{cccc}
f_{11}^{(2.1)} & f_{12}^{(2.1)} & \ldots & f_{1 r}^{(2.1)} \\
f_{21}^{(2.1)} & f_{22}^{(2.1)} & \ldots & f_{2 r}^{(2.1)} \\
\vdots & \vdots & \ddots & \vdots \\
f_{r 1}^{(2.1)} & f_{r 2}^{(2.1)} & \vdots & f_{r r}^{(2.1)}
\end{array}\right]
$$

são as matrizes de densidades espectrais cruzadas dos elementos do vetor $\left\{\mathbf{X}^{(1)}(t)\right\}$ com os elementos do vetor $\left\{\mathbf{X}^{(2)}(t)\right\}$.

Algumas propriedades dessas matrizes são dadas por:

1. $\mathbf{f}_{1,2}(\lambda)=\mathbf{f}_{2,1}^{*}(\lambda)=\overline{\mathbf{f}_{2,1}(\lambda)^{\prime}}$.

2. Os elementos de Diag $\left[\mathrm{f}_{1,1}(\lambda)\right]$ e Diag $\left[\mathrm{f}_{2,2}(\lambda)\right]$ representam as funções de densidades espectrais, na freqüência $\lambda$, de cada série contida em $\left\{\mathbf{X}^{(1)}(t)\right\}$ e $\left\{\mathbf{X}^{(2)}(t)\right\}$, respectivamente.

Para saber se as duas séries multivariadas estacionárias $\left\{\mathbf{X}^{(1)}(t)\right\}$ e $\left\{\mathbf{X}^{(2)}(t)\right\}$ são geradas pelo mesmo processo estacionário, Carmona e Wang(1996) propuseram dois testes no domínio das freqüências, um para o caso de séries independentes e outro para o caso de séries dependentes e que, basicamente, são uma generalização da idéia do teste das somas acumuladas proposto por Coates e Diggle(1986) para o caso univariado.

O objetivo é comparar as matrizes de densidade espectral, ou seja, o teste de interesse é

$$
\begin{array}{lll}
H_{0}: \mathbf{f}_{1,1}(\lambda) \equiv \mathbf{f}_{2,2}(\lambda) & 0<\lambda<\pi \\
H_{A}: \mathbf{f}_{1,1}(\lambda) \not \equiv \mathbf{f}_{2,2}(\lambda) & \text { para algum } 0<\lambda<\pi .
\end{array}
$$

Sem perda de generalidade, assume-se que o número de observações $T$ é um múltiplo de $L, \quad T=2 \mathrm{Lm}$. Considera-se também o estimador suavizado da matriz de densidade espectral $\mathbf{f}$ da forma

$$
\widehat{\mathbf{f}}\left(\lambda_{j}\right)=\frac{1}{L} \sum_{l=1}^{L} d_{X}^{(T)}\left(\lambda_{j, l}\right) d_{X}^{(T)}\left(\lambda_{j, l}\right)^{*}
$$


onde $d_{X}^{(T)}\left(\lambda_{j, l}\right)$ é a transformada de Fourier discreta da série $2 r$-variada $\{\mathbf{X}(t)\}$ e $\left\{\lambda_{j, l}: l=1, \ldots, L\right\}$ é um conjunto de $L$ freqüências que convergem para a freqüência $\lambda_{j}, \quad j=1, \ldots, m$.

Segundo o Teorema $3.8, \widehat{\mathbf{f}}\left(\lambda_{1}\right), \ldots, \widehat{\mathbf{f}}\left(\lambda_{m}\right)$, quando $T \rightarrow \infty$ e $L$ fixo, convergem em distribuição para

$$
\left[\widehat{\mathbf{f}}\left(\lambda_{1}\right), \ldots, \widehat{\mathbf{f}}\left(\lambda_{m}\right)\right] \stackrel{\mathrm{D}}{\longrightarrow}\left[L^{-1} W_{2 r}^{c}\left(L, \mathbf{f}\left(\lambda_{1}\right)\right), \ldots, L^{-1} W_{2 r}^{c}\left(L, \mathbf{f}\left(\lambda_{m}\right)\right)\right]
$$

independentes. Também,

$$
\left[\widehat{\mathbf{f}_{\mathbf{i}, \mathbf{j}}}\left(\lambda_{1}\right), \ldots, \widehat{\mathbf{f}_{\mathbf{i}, \mathbf{j}}}\left(\lambda_{m}\right)\right] \stackrel{\mathrm{D}}{\longrightarrow}\left[L^{-1} W_{r}^{c}\left(L, \mathbf{f}_{\mathbf{i}, \mathbf{j}}\left(\lambda_{1}\right)\right), \ldots, L^{-1} W_{r}^{c}\left(L, \mathbf{f}_{\mathbf{i}, \mathbf{j}}\left(\lambda_{m}\right)\right)\right], \quad i, j=1,2
$$

independentes.

\subsubsection{Comparação de Séries no Caso de Independência (Carmona e Wang)}

Uma primeira situacão que simplifica um pouco o problema, é considerar que as duas séries $r$-variadas $\left\{\mathbf{X}^{(1)}(t)\right\}$ e $\left\{\mathbf{X}^{(2)}(t)\right\}$ são independentes. Neste caso

$$
\mathbf{f}_{1,2}(\lambda) \equiv \mathbf{f}_{2,1}(\lambda) \equiv \mathbf{0}
$$

e a matriz de densidade espectral $f(\lambda)$ é uma matriz bloco diagonal.

Da independência das séries segue-se que $\widehat{f}_{1,1}$ e $\widehat{f}_{2,2}$ são construídas de amostras independentes implicando que $\widehat{\mathbf{f}}_{1,1}$ e $\widehat{\mathbf{f}}_{2,2}$ também são independentes. Logo as $m$ matrizes de dimensão $\mathrm{rxr}, M_{1}\left(\lambda_{j}\right)$ e $M_{2}\left(\lambda_{j}\right)$, definidas por

$$
\begin{aligned}
M_{1}\left(\lambda_{j}\right) & =\mathbf{f}_{1,1}^{-1 / 2}\left(\lambda_{j}\right) \widehat{\mathbf{f}}_{1,1}\left(\lambda_{j}\right) \mathbf{f}_{1,1}^{-1 / 2}\left(\lambda_{j}\right) \\
\text { e } \quad M_{2}\left(\lambda_{j}\right) & =\mathbf{f}_{2,2}^{-1 / 2}\left(\lambda_{j}\right) \widehat{\mathbf{f}}_{2,2}\left(\lambda_{j}\right) \mathbf{f}_{2,2}^{-1 / 2}\left(\lambda_{j}\right)
\end{aligned}
$$

$j=1, \ldots, m$, formam amostras independentes de matrizes independentes com distribuição $\mathrm{W}_{r}^{c}(L, \mathbf{I})$ onde $\mathbf{I}$ representa a matriz identidade de dimensão $r$. Para justificar este resultado observe que

$$
\begin{aligned}
\mathrm{E}\left[\mathrm{f}_{1,1}^{-1 / 2}\left(\lambda_{j}\right) \widehat{\mathrm{f}}_{1,1}\left(\lambda_{j}\right) \mathbf{f}_{1,1}^{-1 / 2}\left(\lambda_{j}\right)\right] & =\mathbf{f}_{1,1}^{-1 / 2}\left(\lambda_{j}\right) E\left[\widehat{\mathrm{f}}_{1,1}\left(\lambda_{j}\right)\right] \mathbf{f}_{1,1}^{-1 / 2}\left(\lambda_{j}\right) \\
& =\mathbf{f}_{1,1}^{-1 / 2}\left(\lambda_{j}\right) \mathbf{f}_{1,1}\left(\lambda_{j}\right) \mathbf{f}_{1,1}^{-1 / 2}\left(\lambda_{j}\right) \\
& =\mathbf{I}_{r}
\end{aligned}
$$


Sob a hipótese $H_{0}$, dada por (5.11), a distribuição da variável aleatória

$$
T\left(\lambda_{j}\right)=\operatorname{traço~}\left[M_{1}\left(\lambda_{j}\right) M_{2}^{-1}\left(\lambda_{j}\right)\right]
$$

independe das matrizes de densidade espectral $f_{1,1}$ e $f_{2,2}$, pois

$$
\begin{aligned}
T\left(\lambda_{j}\right) & =\operatorname{traço}\left[M_{1}\left(\lambda_{j}\right) M_{2}^{-1}\left(\lambda_{j}\right)\right] \\
& =\operatorname{traço}\left[\mathbf{f}_{1,1}^{-1 / 2}\left(\lambda_{j}\right) \widehat{\mathbf{f}}_{1,1}\left(\lambda_{j}\right) \mathbf{f}_{1,1}^{-1 / 2}\left(\lambda_{j}\right) \mathbf{f}_{2,2}^{-1 / 2}\left(\lambda_{j}\right) \widehat{\mathbf{f}}_{2,2}^{-1}\left(\lambda_{j}\right) \mathbf{f}_{2,2}^{-1 / 2}\left(\lambda_{j}\right)\right] \\
& =\operatorname{traço}\left[\widehat{\mathrm{f}}_{1,1}\left(\lambda_{j}\right) \widehat{\mathbf{f}}_{2,2}^{-1}\left(\lambda_{j}\right)\right] .
\end{aligned}
$$

Se $F_{r, L}$ representa a distribuição da estatística $T\left(\lambda_{j}\right)$, então esta distribuição independe da freqüência $\lambda_{j}$, pois $F_{r, L}$ é a distribuição do traço da matriz aleatória $M_{1} M_{2}^{-1} \operatorname{com} M_{1}$ independente de $M_{2}$ e cada uma com distribuição $\mathrm{W}_{r}^{c}(L, \mathbf{I})$.

O teste proposto por Carmona e Wang(1996), para testar a hipótese $H_{0}$ dada por (5.11), baseia-se na estatística $T\left(\lambda_{j}\right)$. Devido ao fato de não conhecermos a distribuição $F_{r, L}$, utiliza-se a técnica Monte Carlo para fazer o teste.

O procedimento do teste é o seguinte:

1. A partir das séries $\left\{\mathbf{X}_{1 t}\right\}$ e $\left\{\mathbf{X}_{2 t}\right\}, t=1, \ldots, T$, estime as matrizes de densidades espectrais $\widehat{\mathbf{f}}_{1,1}\left(\lambda_{j}\right)$ e $\widehat{\mathbf{f}}_{2,2}\left(\lambda_{j}\right), j=1, \ldots, m$.

2. Calcule as estatísticas $T\left(\lambda_{1}\right), \ldots, T\left(\lambda_{m}\right)$, onde $T\left(\lambda_{j}\right)=\operatorname{traço}\left[\widehat{\mathrm{f}}_{1,1}\left(\lambda_{j}\right) \widehat{\mathbf{f}}_{2,2}^{-1}\left(\lambda_{j}\right)\right]$, $j=1, \ldots, m$.

3. Gere independentemente as matrizes $M_{1}\left(\lambda_{j}\right)$ e $M_{2}\left(\lambda_{j}\right), j=1, \ldots, m$, da distribuição $\mathrm{W}_{r}^{c}(L, \mathrm{I})$.

4. Calcule as estatísticas do tipo Monte Carlo, $T^{*}\left(\lambda_{1}\right), \ldots, T^{*}\left(\lambda_{m}\right)$, onde $T^{*}\left(\lambda_{j}\right)=$ traço $\left[M_{1}\left(\lambda_{j}\right) M_{2}^{-1}\left(\lambda_{j}\right)\right], \quad j=1, \ldots, m$.

5. Aplique um teste de Kolmogorov-Smirnov para as duas amostras de traços $T\left(\lambda_{1}\right), \ldots, T\left(\lambda_{m}\right)$ e $T^{*}\left(\lambda_{1}\right), \ldots, T^{*}\left(\lambda_{m}\right)$ e rejeite a hipótese nula ao nível de significância $\alpha$ se o nível descritivo do teste é menor que $\alpha$. Daqui em diante este teste é denominado $T_{K S}$.

\section{Observação}

Os autores também sugerem usar a estatística $D\left(\lambda_{j}\right)=\operatorname{Det}\left[M_{1}\left(\lambda_{j}\right) M_{2}^{-1}\left(\lambda_{j}\right)\right]$ onde Det indica o determinante de $M_{1}\left(\lambda_{j}\right) M_{2}^{-1}\left(\lambda_{j}\right)$. A vantagem de utilizar o traço é devido a maior simplicidade dos cálculos envolvidos. 


\subsubsection{Comparação de Séries no Caso de Dependência (Car- mona e Wang)}

Neste caso $f_{2,1}\left(\lambda_{j}\right) \not \equiv 0$ mas agora, por conveniência, restringe-se a estrutura de coerência, considerando-se que para todo $\lambda_{j}, j=1, \ldots, m$, as matrizes de coerência

$$
\mathbf{f}_{2,2}^{-1 / 2}\left(\lambda_{j}\right) \mathbf{f}_{2,1}\left(\lambda_{j}\right) \mathbf{f}_{1,1}^{-1 / 2}\left(\lambda_{j}\right)
$$

permanecem constantes. Assim, supõe-se a existência de uma matriz de constantes $\mathbf{F}$, de dimensão $r \times r$, tal que

$$
\mathbf{F} \equiv \mathbf{f}_{2,2}^{-1 / 2}(\lambda) \mathbf{f}_{2,1}(\lambda) \mathbf{f}_{1,1}^{-1 / 2}(\lambda), \quad \text { para toda freqüência } \lambda .
$$

Segundo os autores, (5.14) deve ser satisfeita sempre que as séries $\left\{\mathbf{X}^{(1)}(t)\right\}$ e $\left\{\mathbf{X}^{(2)}(t)\right\}$ sigam um modelo linear.

Como foi especificado anteriormente, a matriz de densidade espectral dos dois processos é dada por

$$
f(\lambda)=\left[\begin{array}{lll}
\mathbf{f}_{1,1}(\lambda) & \vdots & \mathbf{f}_{1,2}(\lambda) \\
\ldots \ldots & \ldots \ldots \ldots \\
\mathbf{f}_{2,1}(\lambda) & \vdots & \mathbf{f}_{2,2}(\lambda)
\end{array}\right]_{(2 \mathbf{r}) \times(2 r)}
$$

satisfazendo

$$
\mathrm{f}_{1,2}(\lambda)=\mathrm{f}_{2,1}^{*}(\lambda)=\overline{\mathbf{f}_{1,2}(\lambda)^{\prime}} \text { e } \overline{\mathrm{f}_{1,2}(\lambda)^{\prime}} \not \equiv 0 .
$$

Sob a hipótese $H_{0}$, dada por (5.11), e a suposição da existência da matriz $\mathbf{F}$, dada por (5.14), a correspondente matriz de coerência é

$$
\mathrm{R}(\lambda)=\left[\begin{array}{lll}
\mathbf{I}_{r} & \vdots & \mathbf{F}^{*} \\
\ldots & \ldots & \ldots \\
\mathbf{F} & \vdots & \mathbf{I}_{r}
\end{array}\right]_{(2 \mathbf{r}) \times(2 \mathbf{r})}
$$

para todo $\lambda, \operatorname{com} \mathbf{I}_{r}$ a matriz identidade de dimensão $r$.

Uma estimativa da matriz $\mathbf{F}$ que leva em consideração pequenas variações das matrizes de coerência nas diferentes freqüências poderia ser dada por

$$
\widehat{\mathbf{F}}=\frac{1}{m} \sum_{j=1}^{m} \widehat{\mathbf{f}}_{2,2}^{-1 / 2}\left(\lambda_{j}\right) \widehat{\mathbf{f}}_{2,1}\left(\lambda_{j}\right) \widehat{\mathbf{f}}_{1,1}^{-1 / 2}\left(\lambda_{j}\right) \text {. }
$$


Substituindo $\mathbf{F}$ por $\widehat{\mathbf{F}}$ podemos estimar a matriz de coerência

$$
\widehat{R}(\lambda)=\left[\begin{array}{lll}
\mathbf{I}_{r} & \vdots & \widehat{\mathbf{F}}^{*} \\
\ldots & \cdots & \ldots \\
\widehat{\mathbf{F}} & \vdots & \mathbf{I}_{r}
\end{array}\right]_{(2 \mathbf{r}) \times(2 \mathbf{r})} .
$$

Para testar a hipótese (5.11) para o caso de séries dependentes, Carmona e Wang(1996) propõem usar o mesmo teste $T_{\mathrm{KS}}$ usado no caso de independência, com a diferença que, no caso de dependência, a distribuição sob $H_{0}$ de $M_{1}$ e $M_{2}$ é desconhecida.

Os autores propõem o seguinte procedimento:

1. A partir das séries $\left\{\mathbf{X}_{1 t}\right\}$ e $\left\{\mathbf{X}_{2 t}\right\}$ estime as matrizes $2 r$-dimensionais de densidades espectrais, $\widehat{\mathbf{f}}\left(\lambda_{j}\right), j=1, \ldots, m$.

2. Calcule as estatísticas $T\left(\lambda_{1}\right), \ldots, T\left(\lambda_{m}\right)$, onde $T\left(\lambda_{j}\right)=\operatorname{traço~}\left[\widehat{\mathrm{f}}_{1,1}\left(\lambda_{j}\right) \widehat{\mathrm{f}}_{2,2}^{-1}\left(\lambda_{j}\right)\right]$, $j=1, \ldots, m$.

3. Gere uma série $2 r$-variada $\{\mathbf{Y}(t)\}=\left\{\mathbf{Y}_{1 t}, \mathbf{Y}_{2 t}\right\}$ de um processo Gaussiano com média zero e matriz de densidade espectral $\widehat{R}(\lambda)$,

$$
\widehat{R}(\lambda)=\left[\begin{array}{lll}
\mathbf{I}_{r} & \vdots & \widehat{\mathbf{F}}^{*} \\
\cdots & \ldots & \ldots \\
\widehat{\mathbf{F}} & \vdots & \mathbf{I}_{r}
\end{array}\right]_{(2 \mathrm{r}) \times(2 \mathbf{r})} .
$$

4. A partir das séries $\left\{\mathbf{Y}_{1 t}\right\}$ e $\left\{\mathbf{Y}_{2 t}\right\}$ estime as matrizes $2 r$-dimensionais de densidades espectrais, $\widehat{\mathrm{g}}\left(\lambda_{j}\right)$, onde $\widehat{\mathrm{g}}$ tem a forma

$$
\widehat{\mathrm{g}}=\left[\begin{array}{lll}
\widehat{\mathrm{g}}_{1,1} & \vdots & \widehat{\mathrm{g}}_{1,2} \\
\ldots & \ldots & \ldots \\
\widehat{\mathrm{g}}_{2,1} & \vdots & \widehat{\mathrm{g}}_{2,2}
\end{array}\right]_{(2 \mathrm{r}) \times(2 \mathrm{r})} .
$$

5. Calcule as estatísticas $T^{*}\left(\lambda_{1}\right), \ldots, T^{*}\left(\lambda_{m}\right)$, onde $T^{*}\left(\lambda_{j}\right)=\operatorname{traço}\left[\widehat{\mathrm{g}}_{1,1}\left(\lambda_{j}\right) \widehat{\mathrm{g}}_{2,2}^{-1}\left(\lambda_{j}\right)\right]$, $j=1, \ldots, m$.

6. Calcule $T_{K S_{\text {obs }}}$, a estatística de Kolmogorov-Smirnov para as duas amostras de traços $T\left(\lambda_{1}\right), \ldots, T\left(\lambda_{m}\right)$ e $T^{*}\left(\lambda_{1}\right), \ldots, T^{*}\left(\lambda_{m}\right)$. 
7. Gere $B$ amostras independentes bootstrap $2 r$-variadas, $\left\{\mathbf{Y}^{(b)}(t), t=1, \ldots, T\right\}$ para $b=1, \ldots, B$.

8. Para cada amostra $\left\{\mathbf{Y}^{(b)}(t), t=1, \ldots, T\right\}$, estime as matrizes espectrais $2 r$-dimensionais denominadas $\widehat{\mathrm{g}}_{b}\left(\lambda_{j}\right), \quad b=1, \ldots, B, \quad j=1, \ldots, m$.

9. Para cada amostra $\left\{\mathbf{Y}^{(b)}(t), t=1, \ldots, T\right\}$, calcule as correspondentes estatísticas $T^{b}\left(\lambda_{1}\right), \ldots, T^{b}\left(\lambda_{m}\right)$, onde $T^{b}\left(\lambda_{j}\right)=\operatorname{traço}\left[\widehat{\mathrm{g}}_{1,1_{b}}\left(\lambda_{j}\right) \widehat{\mathrm{g}}_{2,2_{b}}^{-1}\left(\lambda_{j}\right)\right]$, $b=1, \ldots, B, \quad j=1, \ldots, m$.

10. Calcule as $B$ estatísticas $T_{K S}^{b}$ de Kolmogorov-Smirnov para as duas amostras de traços, $T^{*}\left(\lambda_{1}\right), \ldots, T^{*}\left(\lambda_{m}\right)$ e $T^{b}\left(\lambda_{1}\right), \ldots, T^{b}\left(\lambda_{m}\right)$ e construa um histograma que será utilizado para calcular o nível descritivo do teste da hipótese (5.11), através da expressão

$$
\alpha^{\prime}=P\left(T_{K S}^{b}>T_{K S_{o b s}}\right)=\frac{\#\left(T_{K S}^{b}>T_{K S_{o b s}}\right)}{B} .
$$

Rejeite a hipótese nula ao nível descritivo $\alpha$ se $\alpha^{\prime}<\alpha$.

Entretanto os programas utilizados pelos autores sugerem o seguinte procedimento:

1. Repita os passos 1 e 2 do procedimento anterior.

2. Gere uma série $2 r$-variada $\{\mathbf{Y}(t)\}=\left\{\mathbf{Y}_{1 t}, \mathbf{Y}_{2 t}\right\}$ de um processo Gaussiano com média zero e matriz de densidade espectral $\widehat{R}(\lambda)$,

$$
\widehat{\mathrm{R}}(\lambda)=\left[\begin{array}{lll}
\mathbf{I}_{r} & \vdots & \widehat{\mathbf{F}}^{*} \\
\ldots & \ldots & \ldots \\
\widehat{\mathbf{F}} & \vdots & \mathbf{I}_{r}
\end{array}\right]_{(2 \mathbf{r}) \times(2 \mathbf{r})}
$$

3. A partir das séries $\left\{\mathbf{Y}_{1 t}\right\}$ e $\left\{\mathbf{Y}_{2 t}\right\}$ estime as matrizes $2 r$-dimensionais de densidades espectrais, $\widehat{\mathrm{g}}\left(\lambda_{j}\right)$, onde $\widehat{\mathrm{g}}$ tem a forma

$$
\widehat{\mathrm{g}}=\left[\begin{array}{lll}
\widehat{\mathrm{g}}_{1,1} & \vdots & \widehat{\mathrm{g}}_{1,2} \\
\ldots & \ldots & \ldots \\
\widehat{\mathrm{g}}_{2,1} & \vdots & \widehat{\mathrm{g}}_{2,2}
\end{array}\right]_{(2 \mathrm{r}) \times(2 \mathrm{r})} .
$$


4. Calcule as estatísticas $T^{*}\left(\lambda_{1}\right), \ldots, T^{*}\left(\lambda_{m}\right)$, onde $T^{*}\left(\lambda_{j}\right)=\operatorname{traço}\left[\widehat{\mathrm{g}}_{1,1}\left(\lambda_{j}\right) \widehat{\mathrm{g}}_{2,2}^{-1}\left(\lambda_{j}\right)\right]$, $j=1, \ldots, m$.

5. Aplique um teste de Kolmogorov-Smirnov para as duas amostras de traços $T\left(\lambda_{1}\right), \ldots, T\left(\lambda_{m}\right)$ e $T^{*}\left(\lambda_{1}\right), \ldots, T^{*}\left(\lambda_{m}\right)$ e rejeite a hipótese $H_{0}$ dada por (5.11) ao nível de significância $\alpha$ se o nível descritivo do teste é menor que $\alpha$. 


\section{Capítulo 6}

\section{Simulações}

A seguir apresentamos algumas simulações com o objetivo de analisar o poder dos testes propostos no capítulo anterior. Para o caso dos testes univariados as simulações também têm como objetivo comparar o poder dos quatro testes.

\subsection{Testes Univariados}

No caso de séries univariadas fizemos algumas simulações considerando processos $\mathrm{AR}(1)$ e MA(1) com inovações ruído branco com distribuição $N(0,1)$.

A comparação dos testes é feita através dos gráficos das curvas do poder estimado, que é a proporção de rejeições da hipótese de igualdade das duas séries.

Para estimar o poder, geramos pares de séries $\operatorname{AR}(1)$ fixando o parâmetro $\phi_{1}$ da primeira série $\left\{X_{1 t}\right\}$ e variando o parâmetro $\phi_{1}$ da segunda série, $\left\{X_{2 t}\right\}$. Do mesmo jeito se fez com as séries MA(1).

Para cada par de séries geramos 1000 amostras independentes, de tamanhos $\mathrm{T}=100, \mathrm{~T}=400$ e $\mathrm{T}=800$ e para cada par de amostras, aplicamos os quatro testes univariados. Utilizando as 1000 réplicas pudemos estimar o poder dos testes ao nível de significância $\alpha$ igual a 0,05 .

No teste de Mélard e Roy(1984), a função $w(\cdot)$ utilizada em (5.10), na estimação dos elementos $\sigma_{l i}$ de $\Sigma$, é a janela de Barttlet modificada. Os pesos $w\left(j b_{n}\right)$ da fórmula (5.11) e que agora denotamos por $w_{j}\left(b_{n, H}\right)$ são definidos por

$$
w_{j}\left(b_{n, H}\right)=\left\{\begin{array}{cl}
1-\frac{|j|}{b_{n, H}} & |j| \leq b_{n, H} \\
0 & |j|>b_{n, H}
\end{array}\right.
$$


onde $b_{n, H}=H n^{1 / 2}, H$ um inteiro positivo geralmente pequeno. Nas nossas simulações e aplicações tomamos $H=1$.

Para os testes no domínio do tempo, escolhemos $J=8$ e $J=7$, para os testes de Mélard e Roy(1984) e de Quenouille(1958), respectivamente, indicando que vamos comparar as 8 primeiras autocovariâncias e as 7 primeiras autocorrelações (sem incluir $\rho_{0}$ ) das duas séries.

Os resultados da simulação estão apresentados nas Tabelas B1 e B2 do Apêndice B.

A Figura 6.1 resume os resultados referentes à comparação de modelos AR(1), onde o parâmetro $\phi_{1}=0,2$ da primeira série permanece fixo enquanto que o parâmetro $\phi_{1}$ da segunda série varia de 0,2 a 0,8 com incrementos de 0,1 .

A Figura 6.2 resume os resultados referentes à comparação de modelos MA(1), onde o parâmetro $\theta_{1}=0,2$ da primeira série permanece fixo enquanto que o parâmetro $\theta_{1}$ da segunda série varia de 0,2 a 0,8 com incrementos de 0,1 .

Observando as Figuras 6.1 e 6.2 podemos tirar algumas conclusões.

As seguintes conclusões se referem ao desempenho dos testes para pequenas amostras, no caso $\mathrm{T}=100$.

1. De acordo com a Figura 6.1.(a), para modelos $\mathrm{AR}(1)$ o desempenho do teste de Quenouille(1958) com $\mathrm{J}$ pequeno( $\mathrm{J}=2)$ se destaca dos demais, entretanto, para modelos MA(1), segundo a Figura 6.2.(a), seu desempenho é equivalente aos testes de Coates e Diggle(1986) da razão da verossimilhança e das somas acumuladas, ambos no domínio das freqüências.

2. Com relação aos dois testes propostos por Coates e Diggle(1986) vemos que o melhor desempenho é o teste da razão da verossimilhança tanto para processos $\mathrm{AR}(1)$ quanto para processos $\mathrm{MA}(1)$.

3. O teste de Mélard e Roy(1984) tem o pior desempenho tanto para os processos $\mathrm{AR}(1)$ quanto para os processos $\mathrm{MA}(1)$ quando $\mathrm{J}$ é grande(J=8), entretanto, como veremos na Figura 6.3, seu desempenho melhora a medida que ulizamos valores menores para J. Nas Figuras 6.1.(a) e 6.2.(a) utilizamos também $\mathrm{J}=3$. Por esta razão não recomendamos a utilização do teste de Mélard e Roy(1984) com J>3 na comparação de séries temporais com modelos bastante simples (no caso AR(1) e MA(1)). 
a) $A R(1), T=100, \alpha=0,05$

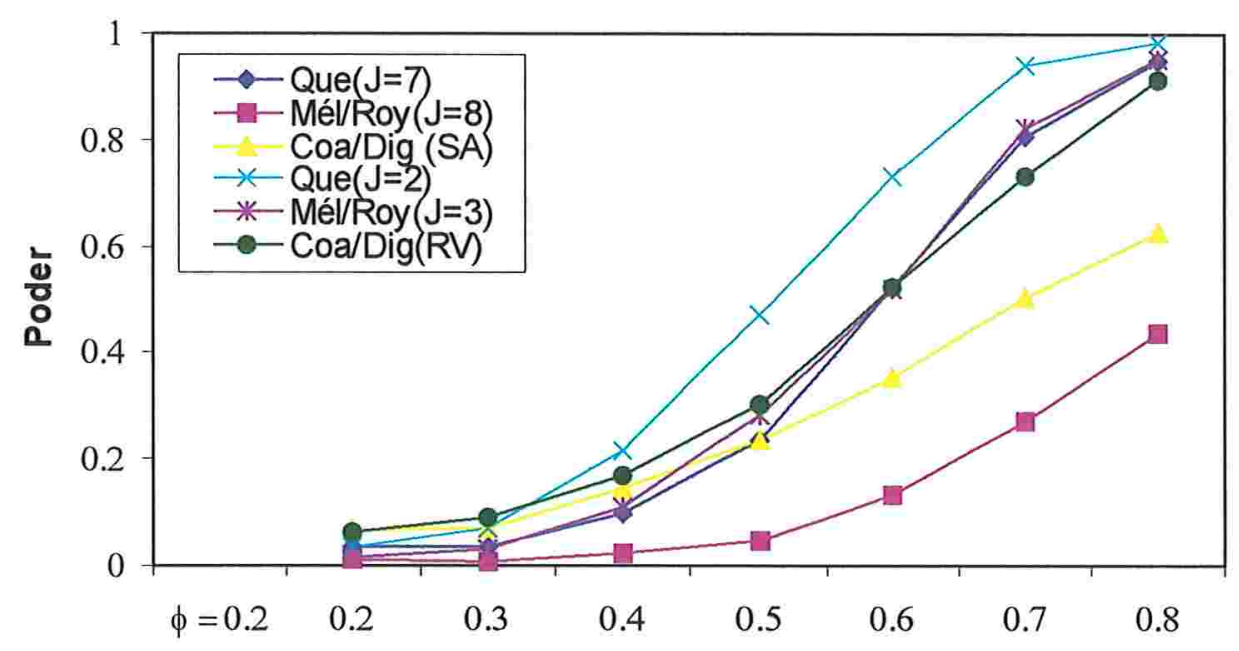

b) $A R(1), T=400, \alpha=0,05$

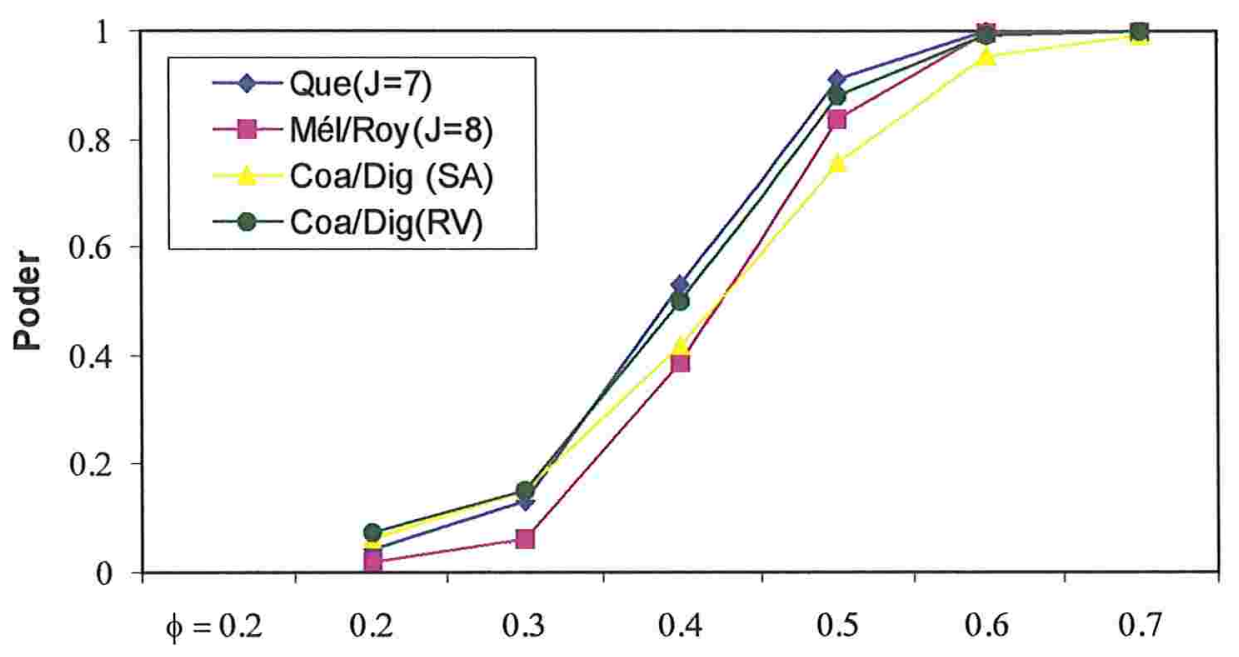

c) $A R(1), T=800, \alpha=0,05$

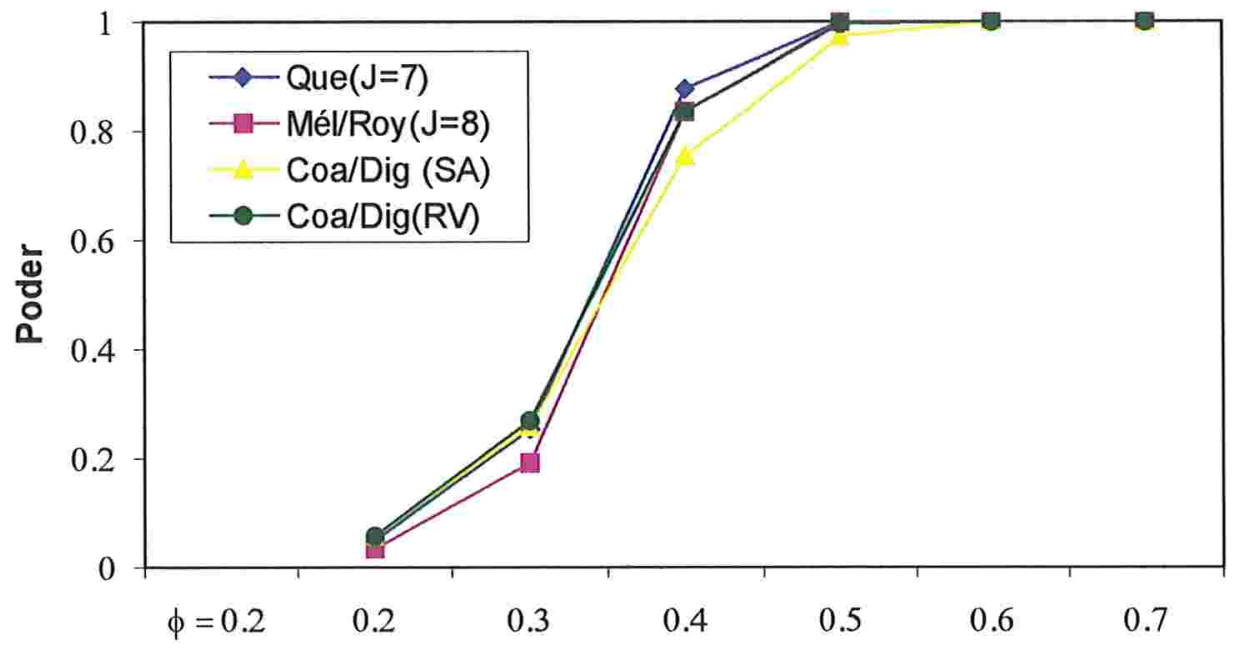

Fig. 6.1. Poder estimado para os testes univariados de modelos $\operatorname{AR}(1) \operatorname{com} \alpha=0,05$. 
a) $M A(1), T=100, \alpha=0,05$

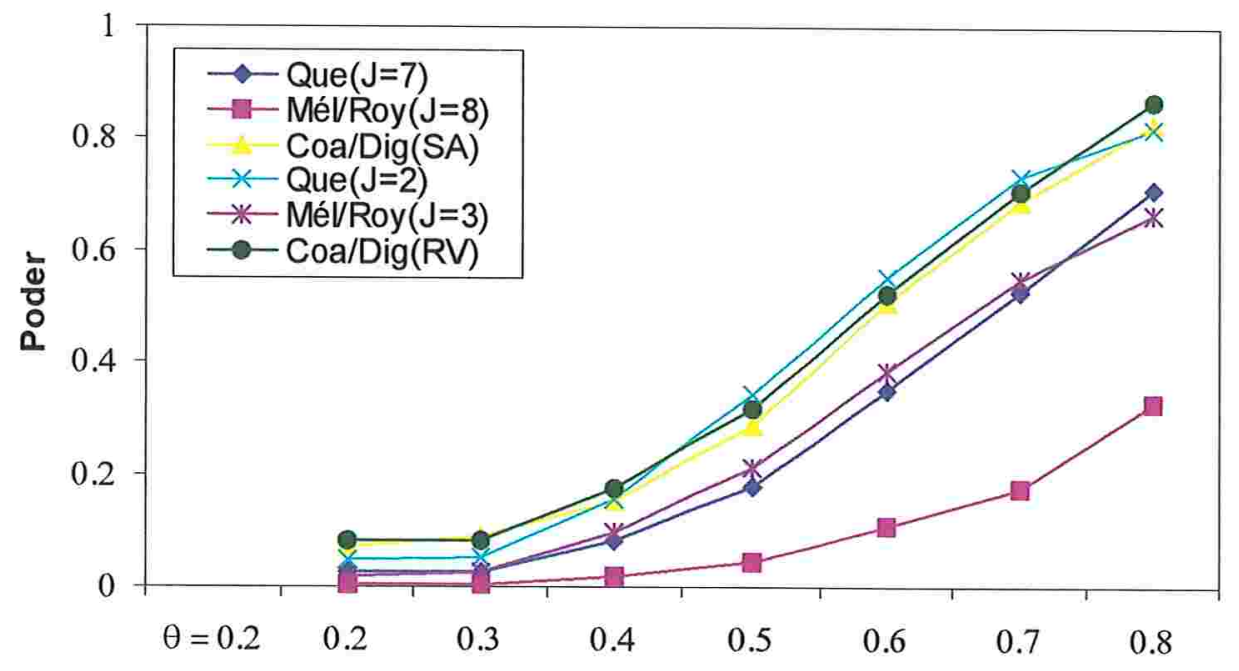

b) MA(1), $T=400, \alpha=0,05$

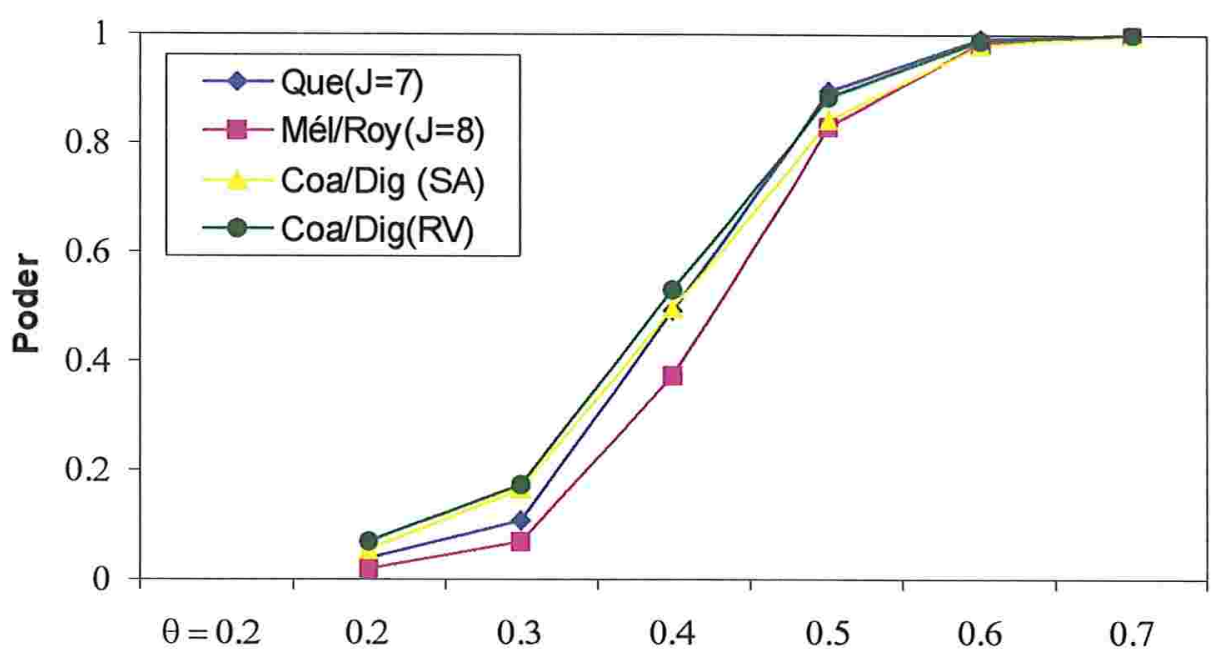

c) $M A(1), T=800, \alpha=0,05$

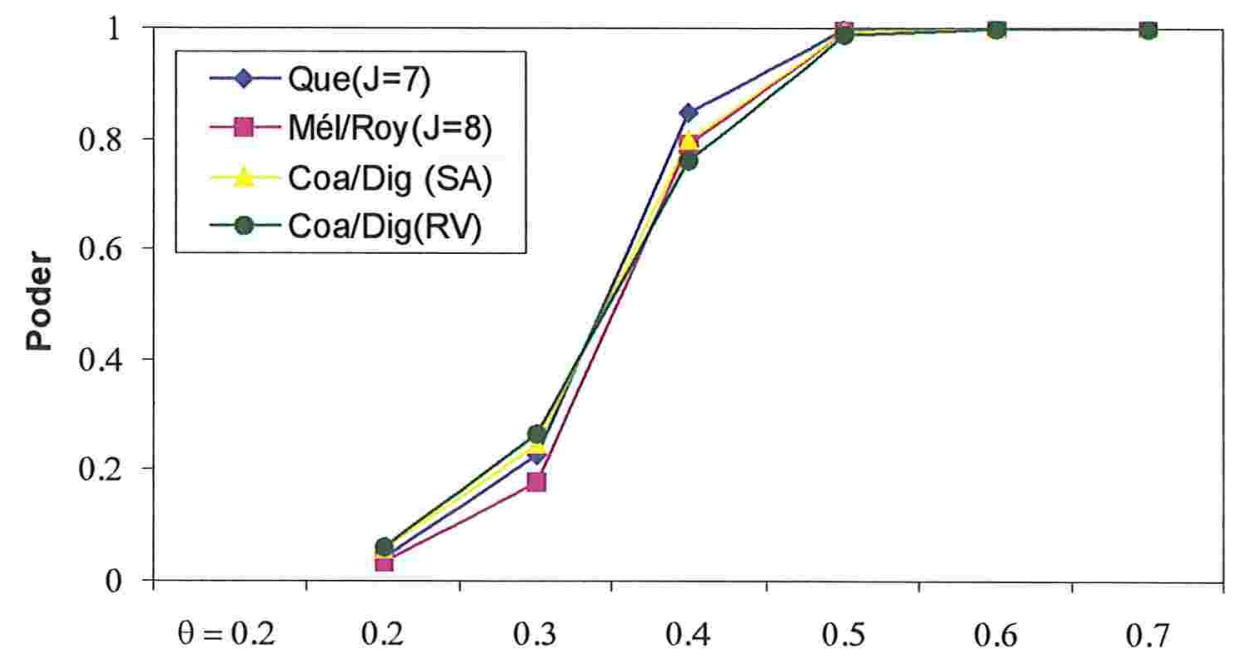

Fig. 6.2. Poder estimado para os testes univariados de modelos $M A(1) \operatorname{com} \alpha=0,05$. 
Em grandes amostras, no caso $\mathrm{T}=400$ e $\mathrm{T}=800$, o desempenho dos quatro testes se aproxima. Assim, em geral, o poder dos testes aumenta com $T$ e com o afastamento entre os dois processos.

Para avaliarmos o efeito do número de autocovariâncias (ou autocorrelações) utilizados nos testes de Mélard e Roy(1984) (ou Quenouille(1958)) estimamos o poder dos testes com valores de $\mathrm{J}=3,5$ e 8 para o caso do teste de Mélard e Roy(1984) e $\mathrm{J}=2$, 4 e 7 para o caso do teste de Quenouille(1958). O número de observações utilizado para cada série foi $\mathrm{T}=100$ e $\alpha=0,05$. Como anteriormente, foram comparados processos $\mathrm{AR}(1)$ e processos $\mathrm{MA}(1)$. Os resultados são apresentados na Tabela B3 do Apêndice B e representados na Figuras 6.3 e 6.4.

Das Figuras 6.3 e 6.4 podemos observar que:

1. Em pequenas amostras, o teste de Quenouille(1958) tem um melhor desempenho que o teste de Mélard e Roy(1984).

2. O poder estimado diminui a medida que $\mathrm{J}$ aumenta, entretanto, este efeito pode ser válido somente em modelos muito simples( $\mathrm{AR}(1)$ e MA(1)).

\subsection{Testes Multivariados}

Para o caso dos testes multivariados apresentamos algumas simulações feitas por Carmona e Wang e que foram obtidas para testar a hipótese $H_{0}$ dada por (5.11) e comparar o desempenho da estatística $T_{K S}$ no caso de séries independentes e séries dependentes. Estes resultados foram tomados do site http://soil.princeton.edu/rcarmona/preprints.html, seção programs, opção spectra.

Carmona e Wang(1996) calcularam o poder do teste baseado na estatística $T_{K S}$ quando a distribuição dos processos são dados por processos bivariados de médias móveis de ordem 2 denotado por $\mathrm{MA}_{2}(2)$. Foi utilizado $\mathrm{T}=1000 \mathrm{com}$ parâmetro de suavização $\mathrm{L}=10$.

A hipótese nula é que o espectro da série bivariada formada pelas duas primeiras componentes da série 4-variada $\{\mathbf{X}(t)\}$ é igual ao espectro da série bivariada formada pelas duas últimas componentes de $\{\mathbf{X}(t)\}$. 
a) Quenouille, AR(1)

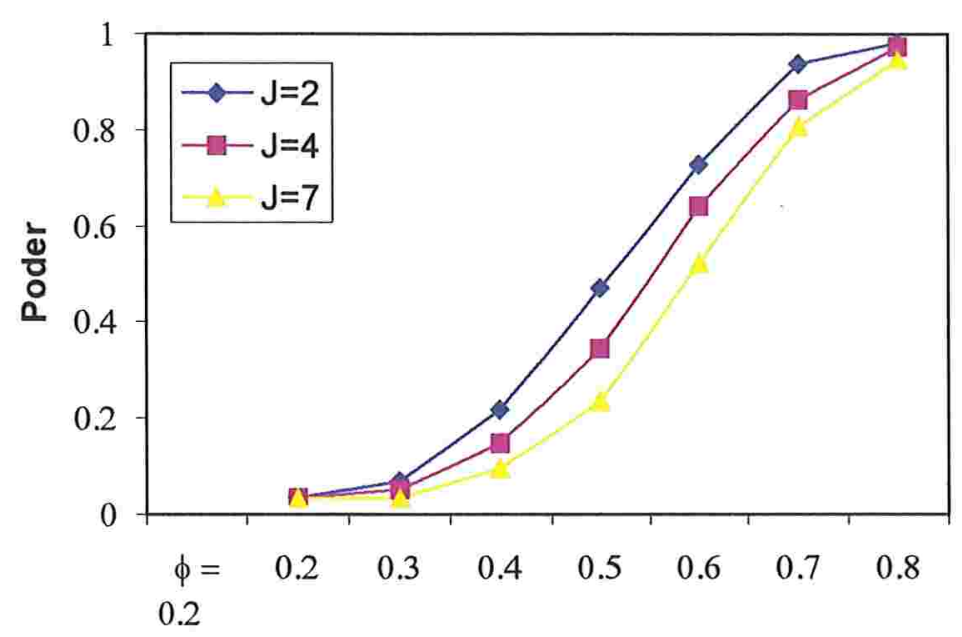

b) Quenouille, MA(1)

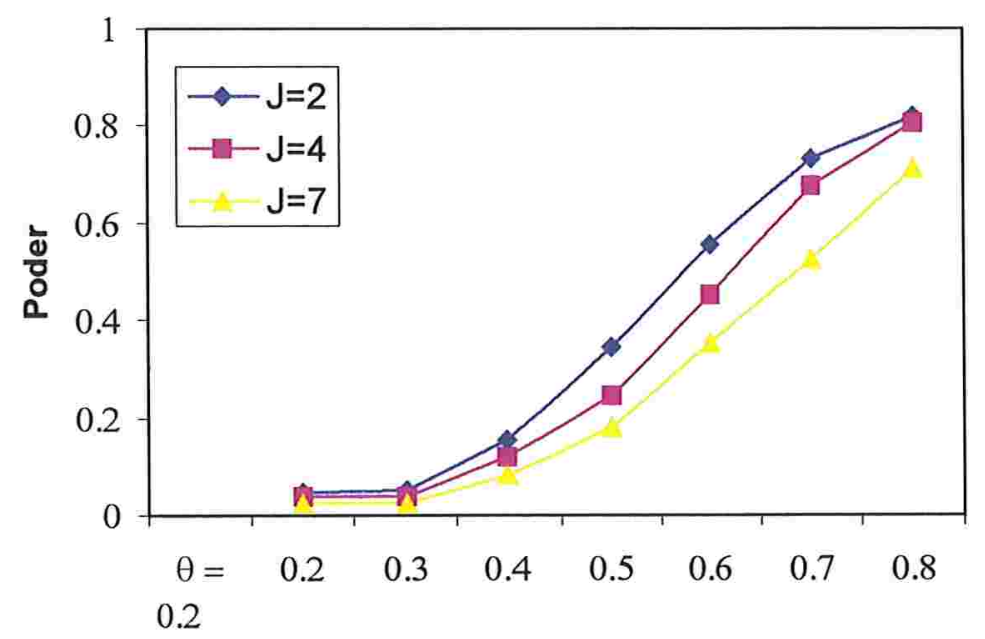

Fig. 6.3. Efeito do parâmetro J no teste de Quenouille em modelos AR(1) e MA(1) usando $\mathrm{T}=100$ e $\alpha=0,05$. 
a) Mélard/Roy, $A R(1)$

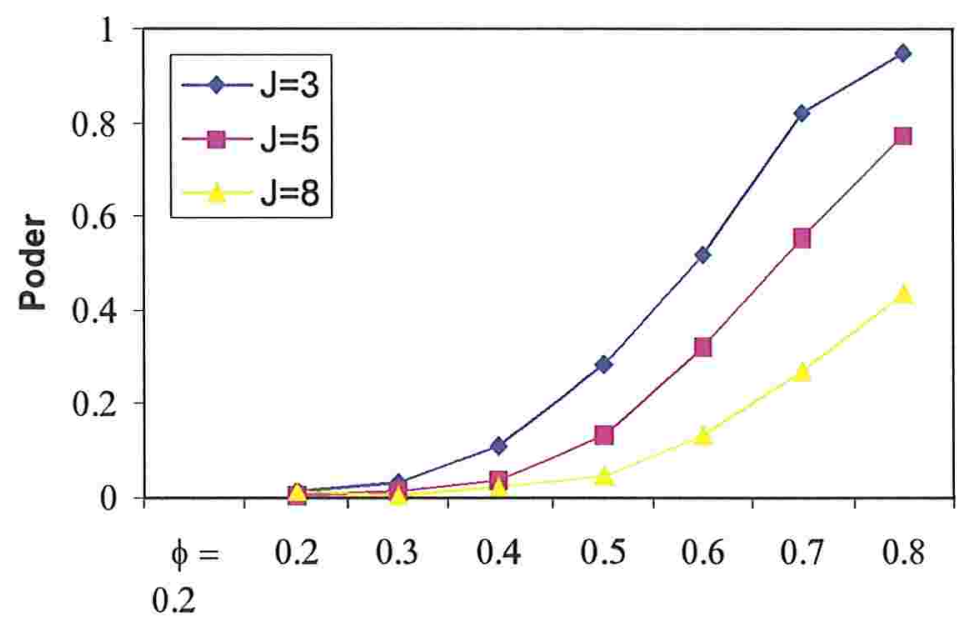

b) Mélard/Roy, MA(1)

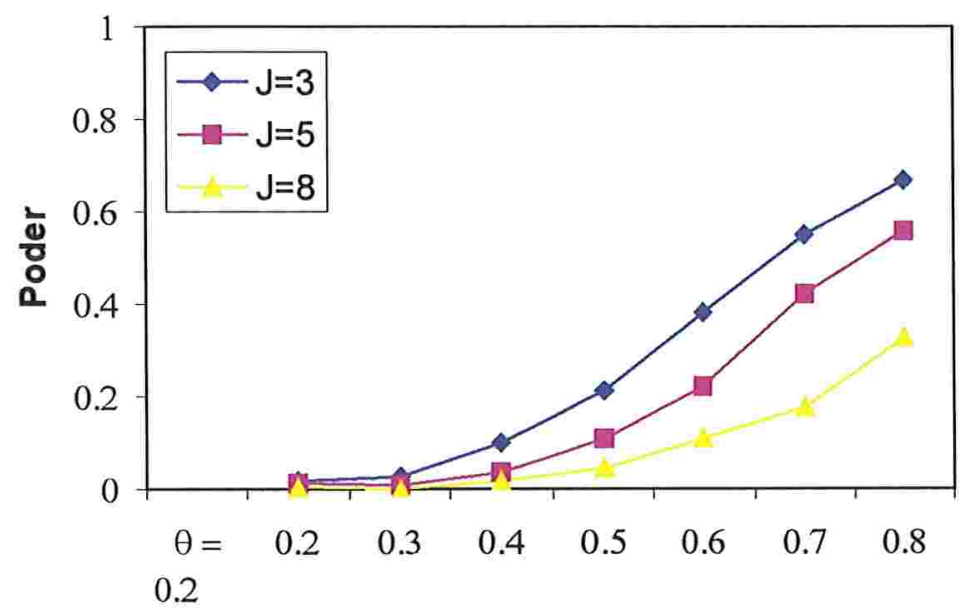

Fig. 6.4. Efeito do parâmetro J no teste de Mélard/Roy em modelos AR(1) e MA(1) usando $\mathrm{T}=100$ e $\alpha=0,05$. 
Para comparar dois processos bivariados de médias móveis de ordem $2, \mathrm{MA}_{2}(2)$, Carmona e Wang(1996) consideraram vários processos 4-variados de médias móveis de ordem $2, \mathrm{MA}_{4}(2)$ da forma

$$
\mathbf{X}(t)=\Theta_{1} \mathbf{a}_{\mathbf{t}-1}+\Theta_{2} \mathbf{a}_{\mathbf{t}-2}+\mathbf{a}_{\mathbf{t}}
$$

com $\left\{\mathbf{a}_{\mathbf{t}}\right\}$ uma seqüência ruído branco com distribuição $N_{4}\left(\mathbf{0}, \mathbf{I}_{\mathbf{4}}\right)$. Para um primeiro exemplo as matrizes de coeficientes $\Theta_{1}$ e $\Theta_{2}$ têm a forma

$$
\Theta_{1}=\left[\begin{array}{llll}
1 & 0 & \theta & 0 \\
0 & 1 & 0 & \theta \\
1 & 0 & 1 & 0 \\
0 & 1 & 0 & 1
\end{array}\right] \quad \text { e } \quad \Theta_{2}=\left[\begin{array}{llll}
1 & 0 & \theta^{\prime} & 0 \\
0 & 1 & 0 & \theta^{\prime} \\
1 & 0 & 1 & 0 \\
0 & 1 & 0 & 1
\end{array}\right]
$$

onde $\theta$ e $\theta^{\prime}$ são parâmetros variando de 0 a 1 com incrementos de 0,2 .

As Tabelas 6.1 e 6.2 fornecem, para cada par de parâmetros, a probabilidade de rejeitar a hipótese nula de igualdade de espectros das duas séries bivariadas $\left\{X_{1, t}, X_{2, t}\right\}$ e $\left\{X_{3, t}, X_{4, t}\right\}$. Observe que as duas séries bivariadas são dependentes e que para $\theta=\theta^{\prime}=1$ elas têm o mesmo espectro.

Tabela 6.1. Poder da Estatística $T_{K S}$ supondo independência entre as séries $\left\{X_{1, t}, X_{2, t}\right\}$ e $\left\{X_{3, t}, X_{4, t}\right\}$.

\begin{tabular}{|l||r|r|r|r|r|r|}
\hline & $\theta^{\prime}=0,0$ & $\theta^{\prime}=0,2$ & $\theta^{\prime}=0,4$ & $\theta^{\prime}=0,6$ & $\theta^{\prime}=0,8$ & $\theta^{\prime}=1,0$ \\
\hline \hline$\theta=0,0$ & 1 & 1 & 1 & 1 & 0,9 & 0,1 \\
\hline$\theta=0,2$ & 1 & 1 & 1 & 1 & 1 & 0,2 \\
\hline$\theta=0,4$ & 1 & 1 & 1 & 1 & 1 & 1 \\
\hline$\theta=0,6$ & 1 & 1 & 1 & 1 & 1 & 1 \\
\hline$\theta=0,8$ & 1 & 1 & 1 & 1 & 1 & 1 \\
\hline$\theta=1,0$ & 0 & 0,3 & 0,9 & 1 & 1 & 1 \\
\hline \hline
\end{tabular}


Tabela 6.2. Poder da Estatística $T_{K S}$ supondo dependência entre as séries $\left\{X_{1, t}, X_{2, t}\right\}$ e $\left\{X_{3, t}, X_{4, t}\right\}$..

\begin{tabular}{|c||c|c|c|c|c|c|}
\hline & $\theta^{\prime}=0,0$ & $\theta^{\prime}=0,2$ & $\theta^{\prime}=0,4$ & $\theta^{\prime}=0,6$ & $\theta^{\prime}=0,8$ & $\theta^{\prime}=1,0$ \\
\hline \hline$\theta=0,0$ & 1 & 1 & 1 & 0,92 & 0,74 & 0,40 \\
\hline$\theta=0,2$ & 1 & 1 & 1 & 0,98 & 0,86 & 0,40 \\
\hline$\theta=0,4$ & 0,98 & 1 & 1 & 1 & 1 & 0,72 \\
\hline$\theta=0,6$ & 0,96 & 1 & 1 & 1 & 1 & 0,90 \\
\hline$\theta=0,8$ & 0,82 & 0,92 & 1 & 1 & 1 & 0,99 \\
\hline$\theta=1,0$ & 0,38 & 0,40 & 0,66 & 0,82 & 0,90 & 0 \\
\hline \hline
\end{tabular}

Podemos observar que a estatística $T_{K S}$ tem um melhor desempenho sob a suposição de dependência entre as duas séries bivariadas.

Um outro exemplo que mostra este melhor desempenho do teste sob a suposição de dependência aparece a seguir, considerando o mesmo modelo (6.1) com matrizes de coeficentes da forma

$$
\Theta_{1}=\left[\begin{array}{llll}
1 & 0 & \theta & 0 \\
0 & 1 & 0 & \theta \\
\theta & 0 & 1 & 0 \\
0 & \theta & 0 & 1
\end{array}\right] \quad \text { e } \quad \Theta_{2}=\left[\begin{array}{cccc}
1 & 0 & \theta^{\prime} & 0 \\
0 & 1 & 0 & \theta^{\prime} \\
\theta^{\prime} & 0 & 1 & 0 \\
0 & \theta^{\prime} & 0 & 1
\end{array}\right]
$$

Neste caso, as duas séries sempre têm o mesmo espectro, com a particularidade que elas são independentes quando $\theta=0$ e $\theta^{\prime}=0$ e sua dependência aumenta com os valores dos parâmetros. O poder do teste para este caso é fornecido pelas Tabelas 6.3 e 6.4 . 
Tabela 6.3. Poder da Estatística $T_{K S}$ supondo independência entre as séries $\left\{X_{1, t}, X_{2, t}\right\}$ e $\left\{X_{3, t}, X_{4, t}\right\}$.

\begin{tabular}{|l||r|r|r|r|r|r|}
\hline & $\theta^{\prime}=0,0$ & $\theta^{\prime}=0,2$ & $\theta^{\prime}=0,4$ & $\theta^{\prime}=0,6$ & $\theta^{\prime}=0,8$ & $\theta^{\prime}=1,0$ \\
\hline \hline$\theta=0,0$ & 0,06 & 0,02 & 0,08 & 0,04 & 0,06 & 0,08 \\
\hline$\theta=0,2$ & 0,08 & 0,06 & 0,04 & 0,06 & 0,02 & 0,04 \\
\hline$\theta=0,4$ & 0,02 & 0,04 & 0,06 & 0,02 & 0,04 & 0,02 \\
\hline$\theta=0,6$ & 0,24 & 0,14 & 0,24 & 0,20 & 0,16 & 0,16 \\
\hline$\theta=0,8$ & 0,94 & 0,92 & 0,90 & 0,82 & 0,92 & 0,92 \\
\hline$\theta=1,0$ & 1 & 1 & 1 & 1 & 1 & 1 \\
\hline \hline
\end{tabular}

Tabela 6.4. Poder da Estatística $T_{K S}$ supondo dependência entre as séries $\left\{X_{1, t}, X_{2, t}\right\}$ e $\left\{X_{3, t}, X_{4, t}\right\}$.

\begin{tabular}{|l||r|r|r|r|r|r|}
\hline & $\theta^{\prime}=0,0$ & $\theta^{\prime}=0,2$ & $\theta^{\prime}=0,4$ & $\theta^{\prime}=0,6$ & $\theta^{\prime}=0,8$ & $\theta^{\prime}=1,0$ \\
\hline \hline$\theta=0,0$ & 0,02 & 0 & 0,04 & 0 & 0 & 0,02 \\
\hline$\theta=0,2$ & 0,06 & 0,02 & 0 & 0,04 & 0,04 & 0 \\
\hline$\theta=0,4$ & 0,02 & 0,02 & 0,02 & 0,04 & 0,04 & 0,04 \\
\hline$\theta=0,6$ & 0,02 & 0,02 & 0,02 & 0,06 & 0,04 & 0 \\
\hline$\theta=0,8$ & 0 & 0,1 & 0,06 & 0,04 & 0,02 & 0,06 \\
\hline$\theta=1,0$ & 0 & 0 & 0 & 0 & 0 & 0 \\
\hline \hline
\end{tabular}

Uma outra observação é o efeito que tem no teste o parâmetro $L$ utilizado na suavização dos periodogramas. Os autores encontraram que para valores pequenos de $L(3 \leq L \leq 6)$ o poder é menor que para valores maiores de $L(8 \leq L \leq 10)$.

Para maiores detalhes consultar Carmona e Wang(1996). 


\section{Capítulo 7}

\section{Aplicações}

Para efeito da aplicação da teoria discutida no Capítulo 5 , selecionamos as variáveis temperatura e salinidade medidas no fundo e na superfície, na estação de monitoramento denominada "Boca de Caño Grande, BCG, da Ciénaga Grande de Santa Marta" (ver Figura 1.1).

Uma análise do tipo descritivo e inferencial é feita às séries, com o objetivo de comparar suas similaridades. A análise inferencial consiste, basicamente, da aplicação dos testes univariados e multivariados expostos no Capítulo 5.

\subsection{Análise Univariada das Séries}

\subsubsection{Análise da Salinidade}

Os registros de porcentagem de salinidade da água medida desde março de 1982 a dezembro de 1995, num total de 166 observações, na estação de monitoramento BCG, no fundo e na superfície da água, aparecem na Figura 7.1(a).

Um teste de igualdade de médias foi feito para as séries pareadas da seguinte forma:

1. Obtivemos a série das diferenças de salinidade $\mathrm{D}, \operatorname{com} \mathrm{D}=$ salinidade no fundo - salinidade na superfície.

2. A média desta diferença foi $\bar{D}=2,9569$ e sua variância $S_{D}^{2}=18,1749$.

3. A variância de $\bar{D}$ é dada por $\operatorname{Var}(\bar{D})=0,10948$. 
4. Para testar a hipótese nula $\mu_{D}=0$ contra a alternativa $\mu_{D} \not \equiv 0$ e usando a estatística $Z=\frac{\bar{D}}{\sqrt{(\operatorname{var}(\bar{D}))}}$ obtivemos o valor $Z=8,9362$. Já que $8,9362>$ 1,96 rejeitamos a hipótese que a média de $D$ é zero(ou equivalentemente que as duas séries de salinidade têm a mesma média) a um nível descritivo de 0,05 .

Na Figura 7.1(a) pode-se observar que as séries são estáveis em média mas não em variância e que as séries apresentam um comportamento sazonal.

Na Figura 7.2 aparecem os periodogramas das séries de salinidade na superfície e no fundo, respectivamente. Um teste de Fisher estabelece que as duas séries apresentam um pico significante na freqüência 0,083333 o que confirma que as séries têm uma componente periódica de 12 meses. Na Tabela 7.1. apresentamos algums valores destes periodogramas. A Figura 7.3 apresenta as funções de autocorrelação das duas séries de salinidade. Nestas duas figuras também se detecta um comportamento sazonal de ordem 12.

Para tornar a série estacionária foi necessário aplicar um logaritmo natural às duas séries e eliminar a sazonalidade determinística através do operador $\left(1-B^{12}\right)$. Feitas estas transformações, prosseguimos a comparação das salinidades usando agora as duas séries estacionárias. 


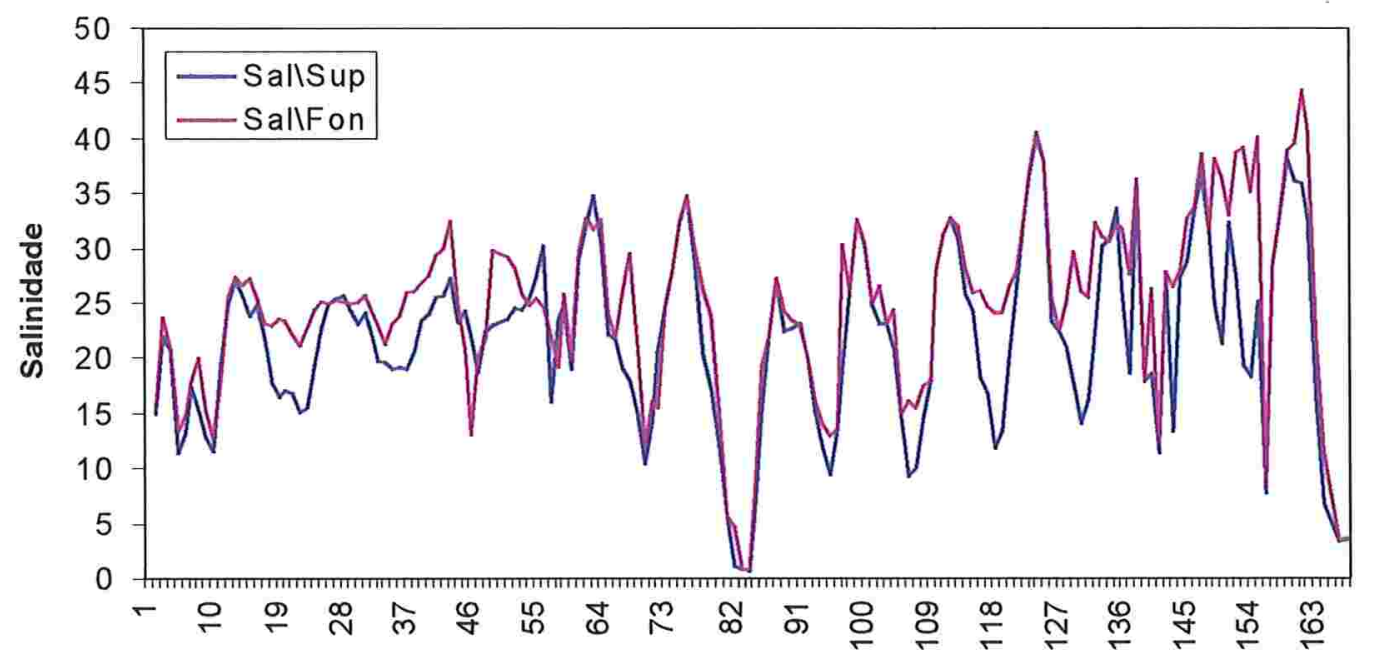

Fig. 7.1. Séries de Salinidade em BCG (a)

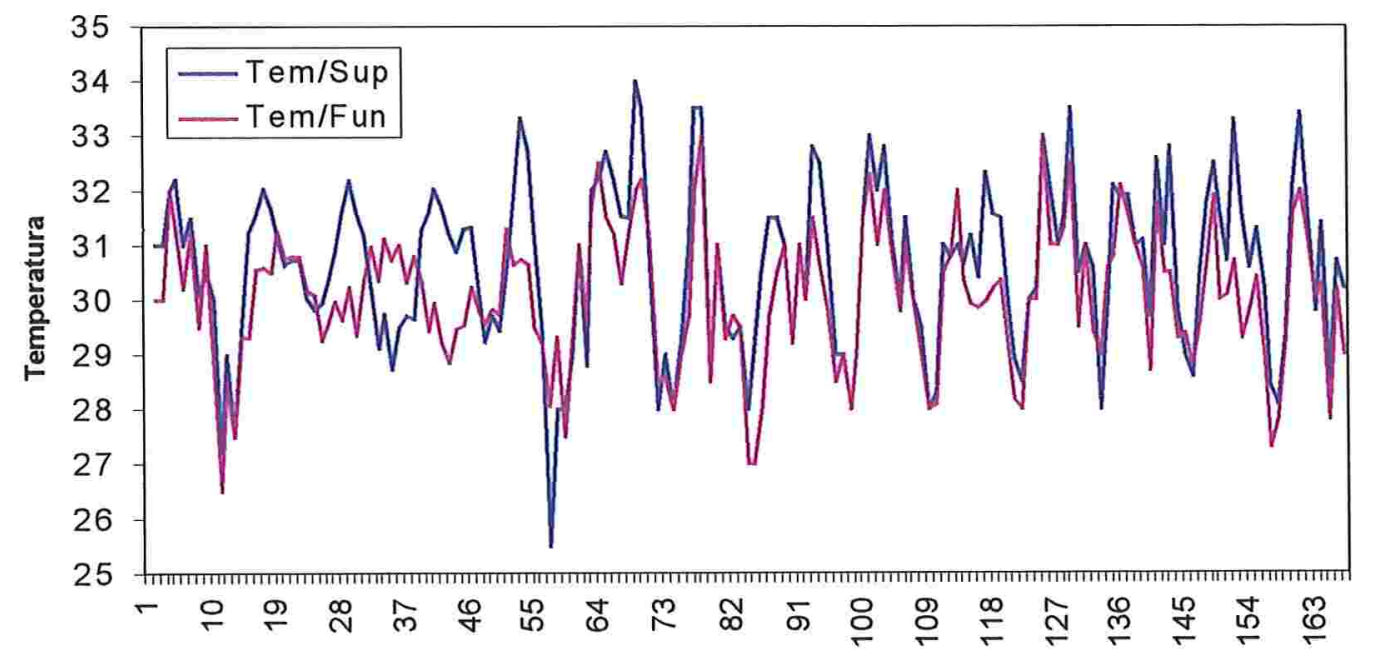

Fig. 7.1. Séries de Temperatura em BCG (b) 
Tabela 7.1. Periodograma das Séries de Salinidade

\begin{tabular}{||l|l||l|l||l|l||}
\hline \hline \multicolumn{2}{||c||}{ Freqüência } & \multicolumn{2}{l||}{ Periodog. Salinidade/Superfície } & \multicolumn{2}{l||}{ Periodog. Salinidade/Fundo } \\
\hline \hline 0,00000 & 0,08928 & 30,11373 & 374,18252 & 37,42883 & 201,98726 \\
0,00595 & 0,09524 & 100,92934 & 9,78786 & 343,69156 & 22,70071 \\
0,01190 & 0,10119 & 315,82221 & 35,43793 & 378,74410 & 4,49549 \\
0,01786 & 0,10714 & 87,18564 & 0,79529 & 40,15367 & 69,80222 \\
0,02381 & 0,11309 & 47,40983 & 13,28454 & 106,60718 & 10,09453 \\
0,02976 & 0,11905 & 20,87852 & 2,95516 & 19,96871 & 10,18584 \\
0,03571 & 0,12500 & 68,88064 & 3,82898 & 191,32228 & 16,69386 \\
0,04167 & 0,13095 & 45,75427 & 4,78697 & 35,07514 & 17,78062 \\
0,04762 & 0,13690 & 30,67030 & 6,28507 & 10,16338 & 29,92099 \\
0,05357 & 0,14286 & 30,77675 & 44,56684 & 112,57564 & 52,70679 \\
0,05952 & 0,14881 & 148,80729 & 3,92874 & 14,85182 & 10,18769 \\
0,06548 & 0,15476 & 181,09948 & 9,92135 & 82,16545 & 5,41783 \\
0,07143 & 0,16071 & 26,63528 & 6,73653 & 36,64088 & 96,72180 \\
0,07738 & 0,16667 & 125,47431 & 87,48681 & 152,61358 & 47,00307 \\
0,08333 & 0,17262 & 1645,64898 & 14,31529 & 1022,55676 & 9,11680 \\
\hline \hline
\end{tabular}
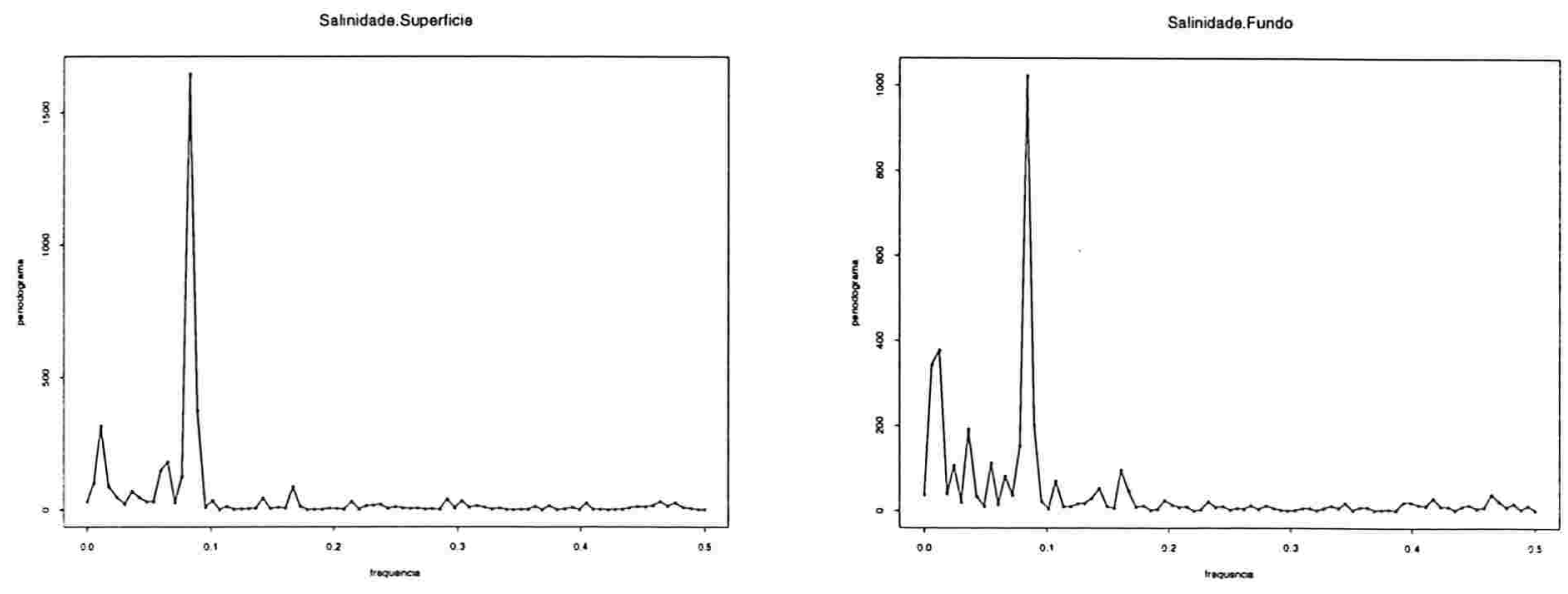

Figura 7.2: Periodogramas da Salinidade na Superfície e no Fundo 

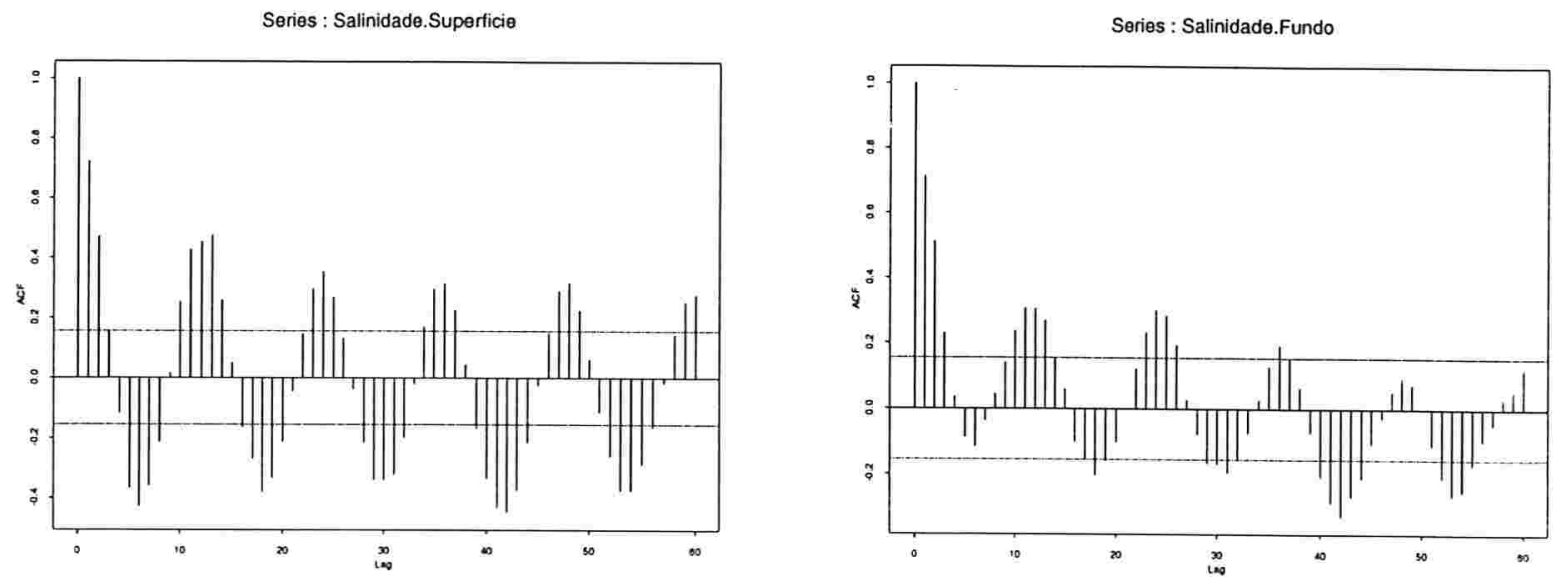

Figura 7.3: FAC das Séries de Salinidade na Superfície e no Fundo

\section{Teste de Quenouille}

Apresentaremos passo a passo o procedimento de Quenouille (Seção 5.1.4) utilizando $\mathrm{J}=15$.

1. As funções de autocorrelação das séries transformadas $\widehat{\rho_{1}}(j)$ e $\widehat{\rho_{2}}(j)$ são apresentadas na Figura 7.4.

Series : Salinidade.Superficie

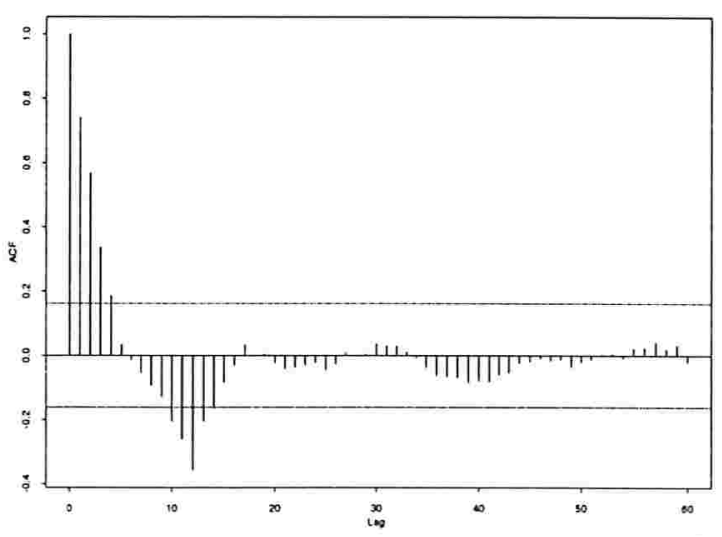

Series : Salinidade.Fundo

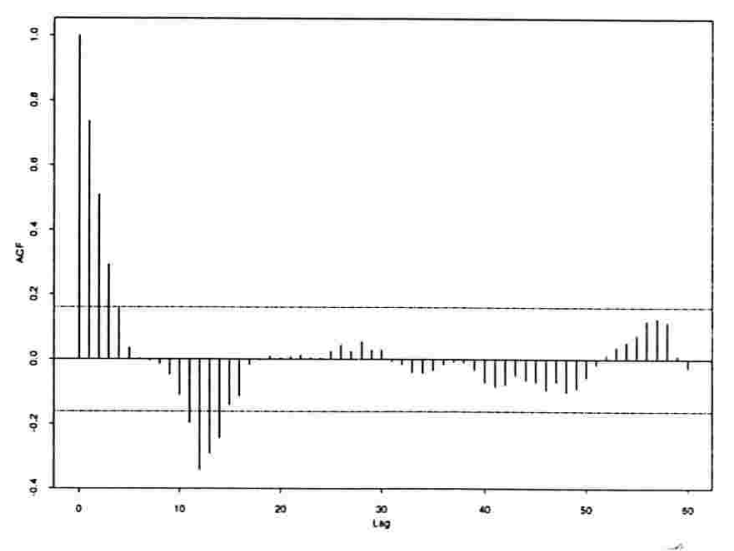

Figura 7.4: FAC das Séries Estacionárias de Salinidade na Superfície e no Fundo

2. A média ponderada das funções de autocorrelação obtidas no passo $1, \widehat{\rho}(j)$, e 
a função de autocorrelação parcial comum $\widehat{\phi}(j)$, são apresentadas na Tabela 7.2 para $j=k=0, \ldots, 29$.

Tabela 7.2. FAC e FAP Comuns às duas Séries de Salinidade

\begin{tabular}{||c|c|c||c|c|c||c|c|c||}
\hline \hline \multicolumn{3}{||c||}{$j$} & \multicolumn{3}{c||}{ FAC comum, $\widehat{\rho}(j)$} & \multicolumn{3}{c||}{ FAP comum, $\widehat{\phi}(j)$} \\
\hline \hline 1 & 11 & 21 & 0,73801 & $-0,22934$ & $-0,01695$ & 0,73807 & 0,08953 & 0,04543 \\
2 & 12 & 22 & 0,53788 & $-0,35216$ & $-0,01177$ & 0,01507 & 0,21981 & 0,00201 \\
3 & 13 & 23 & 0,31424 & $-0,24891$ & $-0,01233$ & 0,17052 & 0,33622 & 0,07601 \\
4 & 14 & 24 & 0,17167 & $-0,20477$ & $-0,00938$ & 0,00521 & 0,08605 & 0,11205 \\
5 & 15 & 25 & 0,03466 & $-0,11349$ & $-0,01007$ & 0,08808 & 0,00886 & 0,18809 \\
6 & 16 & 26 & $-0,00502$ & $-0,07259$ & 0,00919 & 0,07056 & 0,02228 & 0,04903 \\
7 & 17 & 27 & $-0,03068$ & 0,00835 & 0,01789 & 0,00073 & 0,02204 & 0,04599 \\
8 & 18 & 28 & $-0,05453$ & $-0,00234$ & 0,02695 & 0,06901 & 0,04953 & 0,04540 \\
9 & 19 & 29 & $-0,08868$ & 0,00759 & 0,01747 & 0,05253 & 0,01140 & 0,04248 \\
10 & 20 & 30 & $-0,15877$ & $-0,00962$ & 0,03406 & 0,14134 & 0,01385 & 0,03290 \\
\hline \hline
\end{tabular}

3. Visto que o intervalo de confiança de $95 \%$ para as funções de autocorrelação parcial comum estimada é $[-0,1612,0,1612]$, então a ordem auto-regressiva estimada, $p$, utilizando $\widehat{\phi}(j)$ é $p=25$ é os coeficientes estimados são dados por:

Tabela 7.3. Estimativas dos Parâmetros do modelo AR(25)

\begin{tabular}{|c|c|c|c|c|c|c|c|c|c|}
\hline \multicolumn{5}{|c|}{$\mathrm{j}$} & \multicolumn{5}{|c|}{ Parâmetro $\widehat{\phi}_{j}$} \\
\hline 1 & 2 & 3 & 4 & 5 & 0,8272 & 0,0436 & 0,0501 & $-0,0311$ & $-0,0654$ \\
\hline 6 & 7 & 8 & 9 & 10 & 0,0198 & 0,0030 & $-0,5678$ & 0,0543 & 0,0872 \\
\hline 11 & 12 & 13 & 14 & 15 & $-0,1268$ & 0,0408 & 0,4867 & $-0,0647$ & 0,0110 \\
\hline 16 & 17 & 18 & 19 & 20 & 0,0510 & $-0,0261$ & $-0,0778$ & 0,0041 & $-0,2637$ \\
\hline 21 & 22 & 23 & 24 & 25 & $-0,1238$ & 0,0248 & $-0,0007$ & 0,0416 & 0,1881 \\
\hline
\end{tabular}


4. Filtrando cada série com o modelo auto-regressivo de ordem $\mathrm{p}=25$, com os coeficientes estimados no passo 3 e calculando as funções de autocorrelação parcial $v_{j}$ e $v_{j}^{\prime}$ das séries residuais, obtemos os resultados apresentados na Tabela 7.4.

Tabela 7.4. Função de Autocorrelação Parcial das Séries Filtradas

\begin{tabular}{||c|c|c||c|c|c||c|c|c||}
\hline \hline \multicolumn{3}{||c|}{$\mathrm{j}$} & \multicolumn{3}{c|}{$v_{j}$} & \multicolumn{3}{|c||}{$v_{j}^{\prime}$} \\
\hline \hline 2 & 6 & 11 & $-0,0001$ & 0,0025 & $-0,0378$ & 0,0416 & 0,0073 & $-0,0160$ \\
2 & 7 & 12 & 0,1378 & 0,0121 & $-0,2243$ & $-0,0023$ & $-0,0074$ & $-0,0522$ \\
3 & 8 & 13 & 0,0045 & $-0,0163$ & 0,1771 & 0,0366 & 0,0014 & $-0,0482$ \\
4 & 9 & 14 & $-0,0274$ & $-0,0197$ & 0,0448 & $-0,0064$ & $-0,0012$ & $-0,0230$ \\
5 & 10 & 15 & 0,0175 & 0,0147 & $-0,0928$ & 0,0263 & 0,0459 & 0,0299 \\
\hline \hline
\end{tabular}

5. Calculando a estatística de Quenouille

$$
S Q=\sum_{j=1}^{15} \frac{\left(v_{j}-v_{j}^{\prime}\right)^{2}}{\frac{1}{154-j}+\frac{1}{154-j}}
$$

onde $n_{1}=n_{2}=154$ obtemos $S Q=8,974348$ e um nível descritivo correspondente de 0,87885 , implicando na aceitação da hipótese de igualdade das funções de autocorrelação das séries de salinidade na superfície e no fundo de BCG.

\section{Teste de Mélard e Roy}

Aplicando o procedimento de Mélard e Roy (Seção 5.1.3) para comparar as funções de autocovariância das séries estacionárias de salinidade e usando $\mathrm{J}=16$, temos que:

1. As funções de autocovariância estimadas para a séries de salinidade transformadas são proporcionais as funções de autocorrelação apresentadas nas Figura 7.4. 
2. A variável $\mathbf{Z}^{(\mathbf{n})^{\prime}}$, de ordem $(1 \times 15)$, é dada por

$$
\begin{aligned}
& \mathbf{Z}^{(\mathbf{n})^{\prime}}=[0,2261,0,1822,0,3575,0,2581,0,1595,0,0031,-0,0631,-0,2008, \\
& -0,3227,-0,3378,-0,4133,-0,3067,-0,1227,0,2983,0,2756,0,1993] .
\end{aligned}
$$

3. A estatística de Mélard e Roy $\mathbf{Q}^{(\mathbf{n})}=\mathbf{Z}^{(\mathbf{n})^{\prime}} \Sigma^{-1} \mathbf{Z}^{(\mathbf{n})^{\prime}}=4,0228$ fornecendo um nível descritivo igual a 0,9988 que nos leva à aceitação da hipótese de igualdade dos vetores de covariância das duas séries de salinidade.

\section{Testes de Coates e Diggle}

a) Teste das Somas Acumuladas

Os passos para a aplicação do teste das somas acumuladas (Seção 5.1.1) são dados por:

1. Os periodogramas das séries transformadas são apresentados na Figura 7.5.
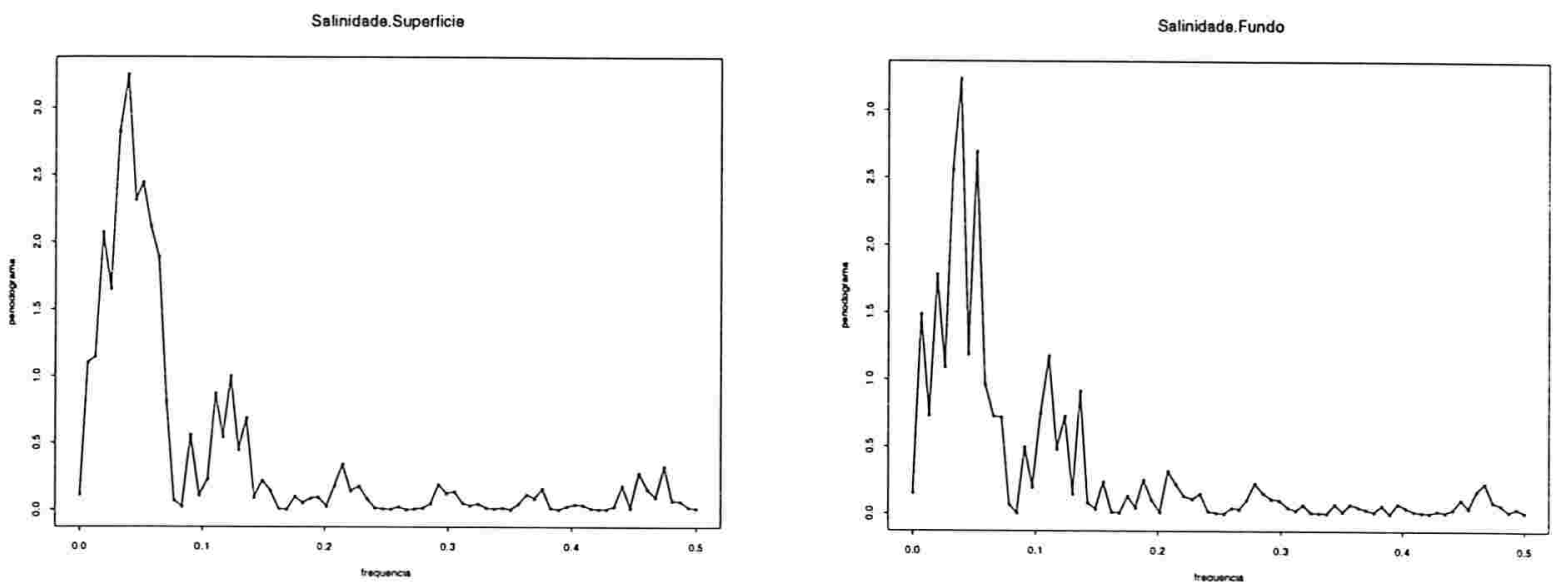

Figura 7.5: Periodogramas das Séries Estacionárias de Salinidade na Superfície e no Fundo

2. A razão dos periodogramas, $J\left(\lambda_{i}\right)$, as estatísticas $z_{i}$, as somas acumuladas $c_{j}$ e as estatísticas $o_{j}$ são apresentadas na Tabela 7.5. 
Tabela 7.5. Algumas Variáveis do Teste das Somas Acumuladas

\begin{tabular}{||c|c|c|c|c||}
\hline \hline $\mathrm{j}$ & $J\left(\lambda_{i}\right)$ & $z_{i}$ & $c_{j}$ & $o_{j}$ \\
\hline \hline 1 & 0,73981 & 0,85513 & 0,85513 & 0,01227 \\
2 & 1,56064 & 0,49515 & 1,35029 & 0,01937 \\
3 & 1,16416 & 0,62002 & 1,97032 & 0,02827 \\
4 & 1,51236 & 0,50755 & 2,47787 & 0,03555 \\
5 & 1,10507 & 0,64443 & 3,12231 & 0,04480 \\
6 & 1,00450 & 0,69090 & 3,81321 & 0,05471 \\
7 & 1,94956 & 0,41405 & 4,22726 & 0,06065 \\
8 & 0,90885 & 0,74207 & 4,96934 & 0,07130 \\
9 & 2,20386 & 0,37414 & 5,34348 & 0,07667 \\
10 & 2,61226 & 0,32411 & 5,66760 & 0,08132 \\
11 & 1,12555 & 0,63575 & 6,30336 & 0,09044 \\
12 & 0,98927 & 0,69855 & 7,00191 & 0,10046 \\
13 & 5,67692 & 0,16224 & 7,16416 & 0,10279 \\
14 & 1,13020 & 0,63382 & 7,79798 & 0,11189 \\
15 & 0,54189 & 1,04569 & 8,84367 & 0,12689 \\
16 & 0,30320 & 1,45816 & 10,30184 & 0,14782 \\
17 & 0,73880 & 0,85592 & 11,15777 & 0,16009 \\
18 & 1,12476 & 0,63608 & 11,79385 & 0,16922 \\
19 & 1,38034 & 0,54491 & 12,33876 & 0,17704 \\
20 & 3,05727 & 0,28298 & 12,62175 & 0,18110 \\
21 & 0,75350 & 0,84463 & 13,46639 & 0,19322 \\
22 & 1,12049 & 0,63788 & 14,10427 & 0,20237 \\
23 & 5,69761 & 0,16170 & 14,26598 & 0,20469 \\
24 & 0,60083 & 0,97996 & 15,24594 & 0,21875 \\
25 & 0,44490 & 1,17793 & 16,42387 & 0,23566 \\
26 & 0,06740 & 2,76228 & 19,18615 & 0,27529 \\
27 & 0,75376 & 0,84443 & 20,03059 & 0,28741 \\
28 & 1,06885 & 0,66040 & 20,69100 & 0,29688 \\
29 & 0,33787 & 1,37616 & 22,06717 & 0,31663 \\
30 & 0,89335 & 0,75111 & 22,81829 & 0,32741 \\
\hline
\end{tabular}


Tabela 7.5. Continuação

\begin{tabular}{||c|c|c|c|c||}
\hline \hline $\mathrm{j}$ & $J\left(\lambda_{i}\right)$ & $z_{i}$ & $c_{j}$ & $o_{j}$ \\
\hline \hline 31 & 2,42673 & 0,34506 & 23,16335 & 0,33236 \\
32 & 0,56104 & 1,02331 & 24,18666 & 0,34704 \\
33 & 1,52930 & 0,50312 & 24,68979 & 0,35426 \\
34 & 1,03838 & 0,67449 & 25,36428 & 0,36394 \\
35 & 1,59732 & 0,48615 & 25,85043 & 0,37091 \\
36 & 0,54596 & 1,04084 & 26,89128 & 0,38585 \\
37 & 0,71817 & 0,87230 & 27,76358 & 0,39837 \\
38 & 0,72438 & 0,86730 & 28,63088 & 0,41081 \\
39 & 1,12869 & 0,63444 & 29,26533 & 0,41991 \\
40 & 0,49091 & 1,11087 & 30,37621 & 0,43585 \\
41 & 0,01158 & 4,46952 & 34,84574 & 0,49999 \\
42 & 0,06041 & 2,86525 & 37,71099 & 0,54110 \\
43 & 0,05574 & 2,94129 & 40,65228 & 0,58330 \\
44 & 0,30704 & 1,44853 & 42,10082 & 0,60409 \\
45 & 1,70495 & 0,46154 & 42,56236 & 0,61071 \\
46 & 1,19277 & 0,60888 & 43,17125 & 0,61945 \\
47 & 2,71898 & 0,31319 & 43,48444 & 0,62394 \\
48 & 1,92716 & 0,41798 & 43,90243 & 0,62994 \\
49 & 0,45146 & 1,16782 & 45,07025 & 0,64669 \\
50 & 4,62557 & 0,19572 & 45,26598 & 0,64950 \\
51 & 1,32706 & 0,56163 & 45,82761 & 0,65756 \\
52 & 0,98646 & 0,69998 & 46,52760 & 0,66760 \\
53 & 0,18655 & 1,85009 & 48,37769 & 0,69415 \\
54 & 0,09748 & 2,42110 & 50,79879 & 0,72889 \\
55 & 0,60137 & 0,97940 & 51,77820 & 0,74294 \\
56 & 2,12435 & 0,38576 & 52,16396 & 0,74848 \\
57 & 2,54939 & 0,33092 & 52,49488 & 0,75323 \\
58 & 9,41906 & 0,10090 & 52,59578 & 0,75467 \\
59 & 0,20818 & 1,75843 & 54,35422 & 0,77991 \\
60 & 0,21765 & 1,72178 & 56,07600 & 0,80461 \\
\hline
\end{tabular}


Tabela 7.5. Continuação

\begin{tabular}{||r|c|c|c|c||}
\hline \hline $\mathrm{j}$ & $J\left(\lambda_{i}\right)$ & $z_{i}$ & $c_{j}$ & $o_{j}$ \\
\hline \hline 61 & 0,33509 & 1,38235 & 57,45835 & 0,82445 \\
62 & 0,84814 & 0,77888 & 58,23724 & 0,83562 \\
63 & 1,83526 & 0,43494 & 58,67219 & 0,84186 \\
64 & 0,64041 & 0,94059 & 59,61278 & 0,85536 \\
65 & 0,42729 & 1,20606 & 60,81884 & 0,87266 \\
66 & 0,23451 & 1,66092 & 62,47976 & 0,89650 \\
67 & 1,96274 & 0,41177 & 62,89153 & 0,90240 \\
68 & 4,97552 & 0,18314 & 63,07467 & 0,90503 \\
69 & 0,13690 & 2,11677 & 65,19145 & 0,93541 \\
70 & 5,97528 & 0,15474 & 65,34619 & 0,93763 \\
71 & 0,91098 & 0,74084 & 66,08704 & 0,94826 \\
72 & 0,41234 & 1,23115 & 67,31819 & 0,96592 \\
73 & 3,65898 & 0,24161 & 67,55980 & 0,96939 \\
74 & 1,01468 & 0,68588 & 68,24569 & 0,97923 \\
75 & 3,81256 & 0,23292 & 68,47861 & 0,98257 \\
76 & 0,42233 & 1,21425 & 69,69287 & 1,00000 \\
\hline \hline
\end{tabular}

3. A estatística de Kolmogorov-Smirnov para comparar a distribuição dos $o_{j}$ fornecidos na última coluna da Tabela $7.5 \mathrm{com}$ a distribuição uniforme em $(0,1)$, forneceu o valor 0,10197 correspondente a um nível descritivo de 0,41652 o que nos leva a aceitar a hipótese de igualdade das funções de densidade espectral das séries de salinidade.

b) Teste da Razão de Verossimilhança

1. Os periodogramas e a razão dos periodogramas $J\left(\lambda_{i}\right)$ aparecem na Figura 7.5 e na segunda coluna da Tabela 7.5 , respectivamente.

2. O vetor das estatísticas $t_{i}=\ln \left(J\left(\lambda_{i}\right)\right), \quad i=1, \ldots, 76$ é dado por 
$\mathrm{t}=[-0,30135,0,44510,0,15200,0,41367,0,09991,0,00449,0,66760,-0,09557$, $0,79021,0,96021,0,11827,-0,01077,1,73640,0,12239,-0,61268,-1,19334$, $0,30272,0,11757,0,32233,1,11752,-0,28302,0,11376,1,74004,-0,50943$, $-0,80988,-2,69705,-0,28267,0,06658,-1,08508,-0,11276,0,88654,-0,57795$, $0,42481,0,03766,0,46833,-0,60519,-0,33103,-0,32243,0,12105,-0,71148$, $-4,45801,-2,80659,-2,88705,-1,18076,0,53353,0,17628,1,00025,0,65604$, $-0,79524,1,53160,0,28296,-0,01362,-1,67903,-2,32808,-0,50854,0,75346$, $0,93585,2,24273,-1,56931,-1,52486,-1,09335,-0,16470,0,60718,-0,44564$, $-0,85027,-1,45024,0,67434,1,60453,-1,98846,1,78763,-0,09322,-0,88590$, $1,29718,0,01458,1,33830,-0,86195$ ]'.

3. As estimativas dos parâmetros $\alpha, \beta$ e $\gamma$ do modelo logístico quadrático (5.6) forneceram os resultados

$$
\widehat{\alpha}=0,49078, \quad \widehat{\beta}=-5,72839, \quad \widehat{\gamma}=10,31825 .
$$

4. O valor da estatística da razão de verossimilhança é $R_{v}^{\prime}=2,24338$ fornecendo um nível descritivo igual a 0,52345 , que nos leva novamente a aceitação da hipótese de igualdade das funcões de densidade espectral da salinidade.

A Tabela 7.6 apresenta um resumo da aplicação dos testes univariados para testar a igualdade das duas séries de salinidade.

Tabela 7.6. Resumo dos Testes Univariados

\begin{tabular}{||l|c||}
\hline \hline \multicolumn{1}{|c|}{ Teste } & Nível Descritivo \\
\hline \hline Quenouille & 0,8788 \\
Mélard e Roy & 0,9988 \\
Coates e Diggle (SA) & 0,4165 \\
Coates e Diggle (RV) & 0,5234 \\
\hline \hline
\end{tabular}

Assim, a um nível de significância de 0,05 os quatro testes estão aceitando a hipótese que as duas séries de salinidade, na superfície e no fundo, na estação de monitoramento BCG são geradas por dois processos com a mesma estrutura de dependência de segunda ordem, porém, com médias diferentes. Uma estimativa das médias dos processos é dada por $\bar{x}_{\text {sup }}=21,7566$ e $\bar{x}_{\text {fundo }}=24,7135$. 


\subsubsection{Análise da Temperatura}

O Gráfico 7.1(b) representa as duas séries de temperatura $\left({ }^{0} C\right)$ em BCG, no fundo e na superfície da água. Observa-se nele que as séries são constantes em média e variância, mas aparentemente percebe-se alguma periodicidade nos dados.

Um teste de igualdade de médias foi feito para as séries pareadas da seguinte forma:

1. Obtivemos a série das diferenças de temperatura D, com D = temperatura na superfície - temperatura no fundo.

2. A média desta diferença foi $\bar{D}=0,5853$ e sua variância $S_{D}^{2}=0,788011$.

3. A variância de $\bar{D}$ é dada por $\operatorname{Var}(\bar{D})=0,004747$.

4. Para testar a hipótese nula $\mu_{D}=0$ contra a alternativa $\mu_{D} \not \equiv 0$ e usando a estatística $Z=\frac{\bar{D}}{\sqrt{(\operatorname{var}(\bar{D}))}}$ obtivemos o valor $\mathrm{Z}=8,49$, o qual nos leva a rejeitar a hipótese que a média de $D$ é zero, ou equivalentemente que as duas séries de temperatura têm a mesma média.

Os periodogramas das séries aparecem na Figura 7.6. A aplicação de um teste de Fisher para o valor do periodograma na freqüência 0.08333 indica que as séries apresentam uma componente periódica de 12 meses. Alguns valores dos periodogramas são apresentados na Tabela 7.7.

Tabela 7.7. Periodogramas das Séries de Temperatura

\begin{tabular}{||l|l||l|l||l|l||}
\hline \hline \multicolumn{2}{||c||}{ Freqüência } & Period. Temperatura/Superfície & Period. Temperatura/Fundo \\
\hline \hline 0,00000 & 0,08928 & 0,00054 & 1,72024 & 0,02102 & 3,04146 \\
0,00595 & 0,09524 & 0,35914 & 0,40595 & 0,16187 & 0,63561 \\
0,01190 & 0,10119 & 0,83283 & 0,01009 & 1,81798 & 0,12373 \\
0,01786 & 0,10714 & 0,40390 & 0,04063 & 0,95185 & 0,37568 \\
0,02381 & 0,11309 & 0,28030 & 1,09224 & 0,89463 & 0,71644 \\
\hline
\end{tabular}


Tabela 7.7. Continuação

\begin{tabular}{||l|l||l|l||l|l||}
\hline \hline \multicolumn{2}{||c||}{ Freqüência } & \multicolumn{1}{|l||}{ Period. Temperatura/Superfície } & Period. Temperatura/Fundo \\
\hline \hline 0,02976 & 0,11905 & 0,83786 & 10,19527 & 6,80338 & 2,71395 \\
0,03571 & 0,12500 & 2,96731 & 12,26685 & 0,67829 & 3,28450 \\
0,04166 & 0,13095 & 4,44447 & 3,35027 & 0,59648 & 0,46440 \\
0,04762 & 0,13690 & 4,53263 & 0,58907 & 0,78645 & 0,41011 \\
0,05357 & 0,14286 & 0,30429 & 0,33585 & 0,80654 & 0,70862 \\
0,05952 & 0,14881 & 3,09521 & 1,52623 & 2,75468 & 0,32209 \\
0,06547 & 0,15476 & 0,16569 & 0,11435 & 4,77002 & 0,14421 \\
0,07143 & 0,16071 & 0,30947 & 0,96857 & 4,78438 & 2,88015 \\
0,07738 & 0,16667 & 11,6752 & 17,23864 & 5,19771 & 11,35694 \\
0,08333 & 0,17262 & 47,7240 & 2,46542 & 23,12778 & 1,56091 \\
\hline \hline
\end{tabular}
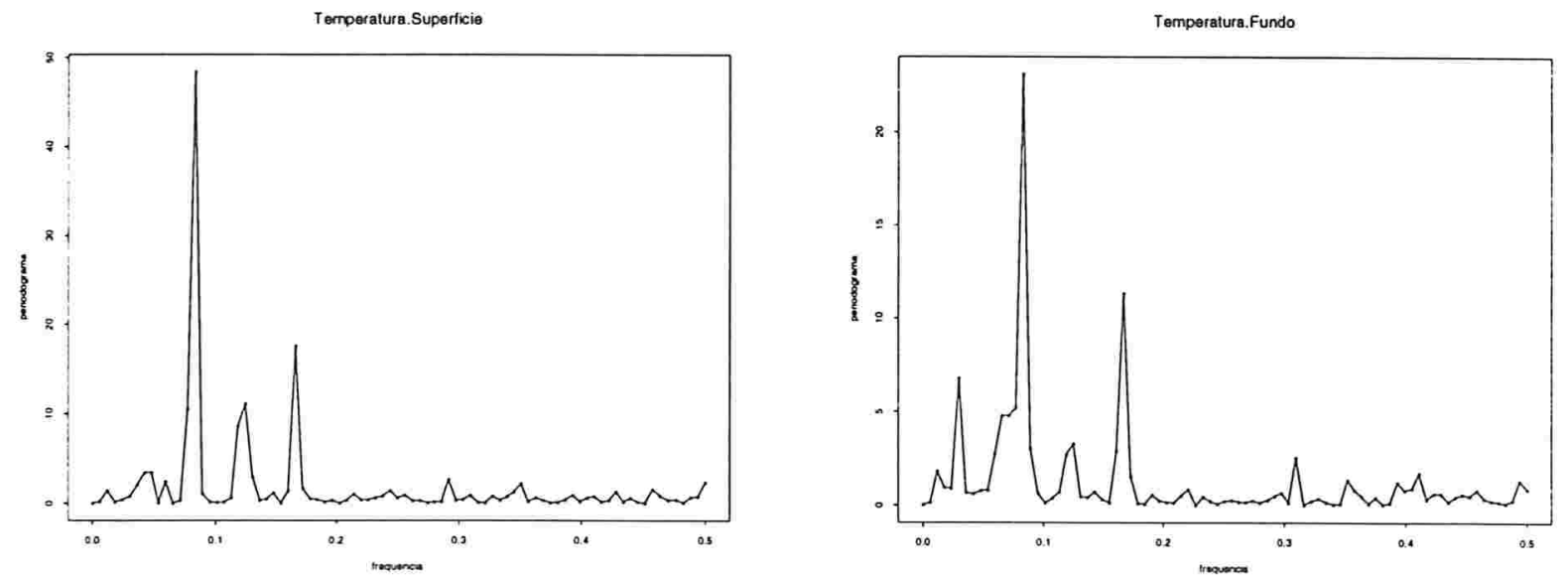

Figura 7.6: Periodogramas das Séries de Temperatura na Superfície e no Fundo

Além desta componente periódica, detectada na Figura 7.6, as funções de autocorrelação que aparecem na Figura 7.7 estabelecem a existência de uma sazonalidade nas duas séries também de período 12 . 
Series : Temperatura.Superlicie

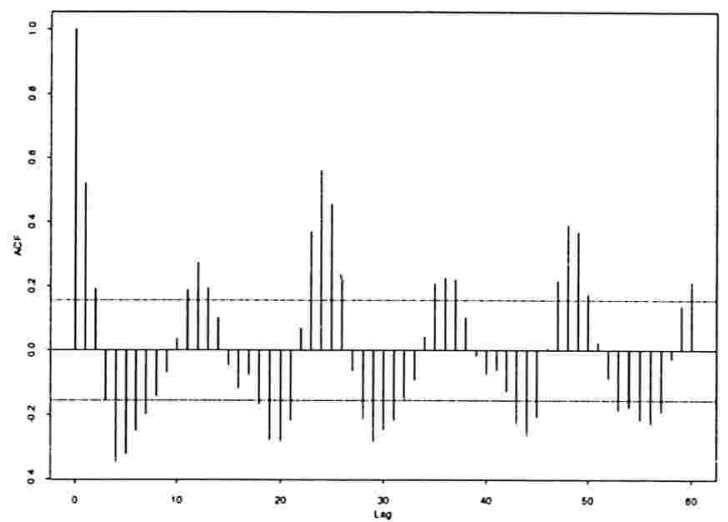

Series: Temperatura Fundo

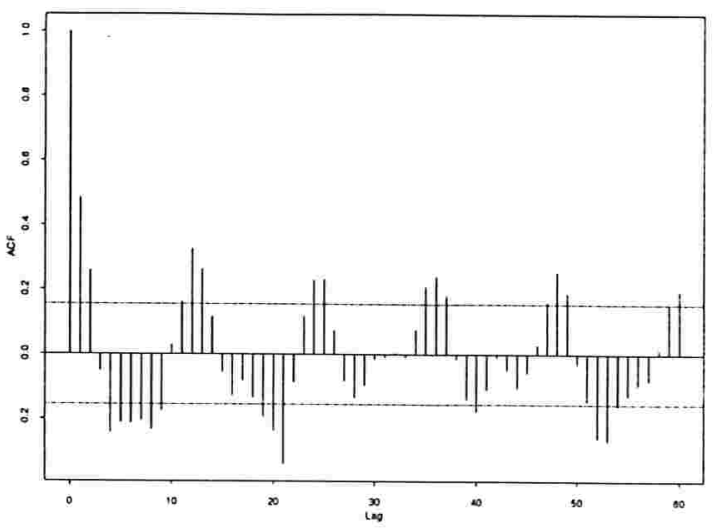

Figura 7.7: FAC das Séries de Temperatura na Superfície e no Fundo

A sazonalidade determinística foi eliminada através do operador $\left(1-B^{12}\right)$ aplicado às duas séries e, uma vez eliminada esta sazonalidade determinística, prosseguimos comparando as séries das primeiras diferenças sazonais.

\section{Teste de Quenouille}

Novamente apresentaremos o procedimento de Quenouille (Seção 5.1.4) para comparar as séries de Temperatura, utilizando $\mathrm{J}=24$.

1. As funções de autocorrelação das séries transformadas, $\widehat{\rho_{1}}(j)$ e $\widehat{\rho_{2}}(j)$ são apresentadas na Figura 7.8.

2. A média ponderada das funções de autocorrelação obtidas no passo $1, \widehat{\rho}(j)$, e a função de autocorrelação parcial comum são apresentadas na Tabela 7.8 .

3. Dado que o intervalo de confiança para os $\widehat{\phi}(j)$ é $[-0,1612,0,1612]$, a ordem auto-regressiva estimada utilizando $\widehat{\phi}(j)$ é $\mathrm{p}=14$ e os coeficientes estimados são dados na Tabela 7.9. 
Series : Temperatura.Superficie

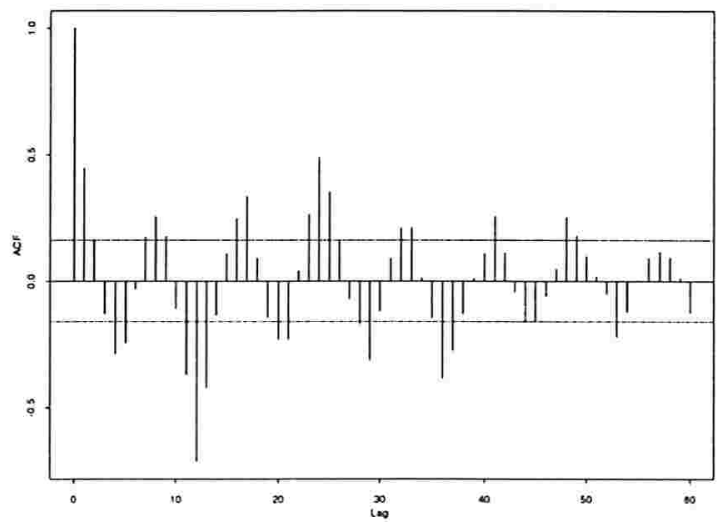

Series: Temperatura.Fundo

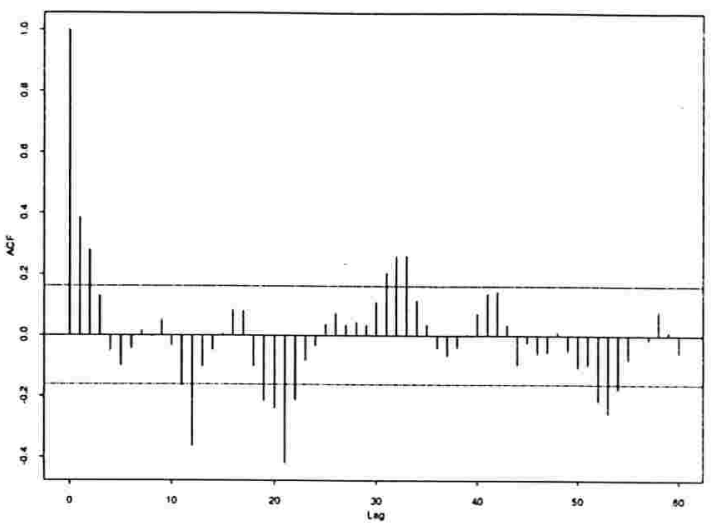

Figura 7.8: FAC das Diferenças Sazonais da Temperatura na Superfície e no Fundo

Tabela 7.8. FAC e FAP Comuns às duas Séries de Temperatura

\begin{tabular}{||c|c|c||c|c|c||c|c|c||}
\hline \hline \multicolumn{3}{||c||}{$j$} & \multicolumn{2}{c||}{ FAC comum, $\widehat{\rho}(j)$} & \multicolumn{2}{c||}{ FAP comum, $\widehat{\phi}(j)$} \\
\hline \hline 1 & 11 & 21 & 0,41618 & $-0,26867$ & $-0,32522$ & 0,41618 & $-0,21135$ & $-0,12666$ \\
3 & 12 & 22 & 0,21983 & $-0,53899$ & $-0,08646$ & 0,05639 & $-0,40131$ & 0,06219 \\
4 & 13 & 23 & $-0,00145$ & $-0,26480$ & 0,09148 & $-0,13498$ & 0,19013 & $-0,02864$ \\
5 & 14 & 24 & $-0,17099$ & $-0,09202$ & 0,22778 & $-0,17091$ & 0,16686 & $-0,11286$ \\
6 & 15 & 25 & $-0,17348$ & 0,05641 & 0,19418 & $-0,02931$ & 0,00374 & 0,06150 \\
7 & 17 & 26 & $-0,03823$ & 0,16601 & 0,11808 & 0,12125 & $-0,10025$ & 0,09353 \\
8 & 18 & 28 & 0,09704 & 0,20747 & $-0,01884$ & 0,12009 & 0,05181 & $-0,02802$ \\
9 & 19 & 29 & 0,12548 & $-0,00568$ & $-0,06295$ & $-0,00690$ & $-0,12898$ & 0,05796 \\
10 & 20 & 30 & $-0,0732$ & $-0,23782$ & $-0,00557$ & $-0,17359$ & $-0,07018$ & $-0,05022$ \\
\hline \hline
\end{tabular}

Tabela 7.9. Estimativas dos Parâmetros do Modelo AR(14)

\begin{tabular}{||lrrrr|llrrr||}
\hline \hline \multicolumn{5}{|c|}{$\mathrm{j}$} & \multicolumn{5}{|c||}{ Parâmetro $\widehat{\phi}_{j}$} \\
\hline \hline 1 & 2 & 3 & 4 & 5 & 0,2812 & 0,2173 & 0,0483 & $-0,1304$ & $-0,0786$ \\
6 & 7 & 8 & 9 & 10 & 0,0258 & 0,0329 & $-0,0184$ & 0,1061 & $-0,0130$ \\
11 & 12 & 13 & 14 & & $-0,1116$ & $-0,4701$ & 0,1379 & 0,1669 & \\
\hline \hline
\end{tabular}


4. Filtrando cada série com o modelo auto-regressivo de ordem $\mathrm{p}=14$, com os coeficientes estimados no passo anterior e calculando as funções de autocorrelação parcial $v_{j}$ e $v_{j}^{\prime}$ das séries residuais, obtemos os resultados apresentados na Tabela 7.10.

Tabela 7.10. Função de Autocorrelação Parcial das Séries Filtradas

\begin{tabular}{||c|c|c||c|c|c||c|c|c||}
\hline \hline \multicolumn{3}{||c||}{$\mathrm{j}$} & \multicolumn{3}{c||}{$v_{j}$} & \multicolumn{3}{c||}{$v_{j}^{\prime}$} \\
\hline \hline 1 & 9 & 17 & $-0,03813$ & $-0,00928$ & 0,17090 & $-0,00394$ & $-0,07676$ & 0,044513 \\
2 & 10 & 18 & $-0,11835$ & $-0,03414$ & 0,02941 & 0,07401 & 0,05493 & $-0,120128$ \\
3 & 11 & 19 & $-0,08271$ & $-0,01071$ & $-0,10226$ & 0,10542 & $-0,02263$ & $-0,148187$ \\
4 & 12 & 20 & $-0,04758$ & $-0,23592$ & $-0,00416$ & $-0,02843$ & $-0,09315$ & $-0,003226$ \\
5 & 13 & 21 & 0,03016 & $-0,13252$ & $-0,09528$ & 0,03561 & 0,03456 & $-0,216427$ \\
6 & 14 & 22 & 0,02458 & $-0,07231$ & $-0,00751$ & $-0,02936$ & 0,02202 & $-0,031818$ \\
7 & 15 & 23 & $-0,01333$ & $-0,02785$ & $-0,00317$ & $-0,05709$ & 0,02745 & $-0,019473$ \\
8 & 16 & 24 & 0,01628 & $-0,11800$ & $-0,09289$ & $-0,08504$ & $-0,05494$ & $-0,277685$ \\
\hline \hline
\end{tabular}

5. A estatística

$$
S Q=\sum_{j=1}^{24} \frac{\left(v_{j}-v_{j}^{\prime}\right)^{2}}{\frac{1}{154-j}+\frac{1}{154-j}}
$$

forneceu o valor $\mathrm{SQ}=18,15516$ correspondente a um nível descritivo de 0,79542, implicando na aceitação da hipótese de igualdade das funções de autocorrelação.

\section{Teste de Mélard e Roy}

Aplicando o procedimento de Mélard e Roy (Seção 5.1.3) para comparar as funções de autocovariância das séries transformadas temos que:

1. As funções de autocovariância estimada para as séries das diferenças sazonais de temperatura são proporcionais as funções de autocorrelação apresentadas na Figura 7.8. 
2. O vetor $\mathbf{Z}^{(\mathbf{n})^{\prime}}$, de ordem $(1 \times 25)$, é dado por:

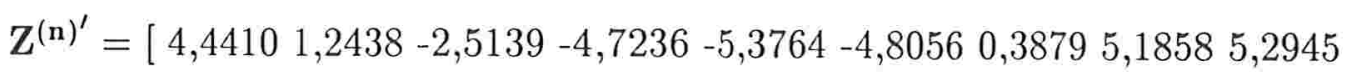

$\begin{array}{llllllllll}2,4903 & 0,7756 & -4,4777 & -7,9249 & -5,5331 & -1,6026 & 2,3773 & 4,3728 & 6,2505 & 2,9080\end{array}$

$-1,1031-0,84752,87452,08807,56799,7113]$.

3. A estatística $\mathbf{Q}^{(\mathbf{n})}=\mathbf{Z}^{(\mathbf{n})^{\prime}} \widehat{\Sigma}^{-1} \mathbf{Z}^{(\mathbf{n})^{\prime}}=41,16934$ fornecendo um nível descritivo igual a 0,02203 que nos leva à rejeição da hipótese de igualdade dos vetores de covariância das duas séries de Temperatura, ao nível de significância de 0,05 .

\section{Testes de Coates e Diggle}

\section{a) Teste das Somas Acumuladas}

Os passos na aplicação do teste das somas acumuladas (Seção 5.1.1) são:

1. Os periodogramas das diferenças sazonais das séries de temperatura são apresentados na Figura 7.9.

2. A razão dos periodogramas, $J\left(\lambda_{i}\right)$, as estatísticas $z_{i}$, as somas acumuladas $c_{j}$ e as estatísticas $o_{j}$ são apresentadas na Tabela 7.9.
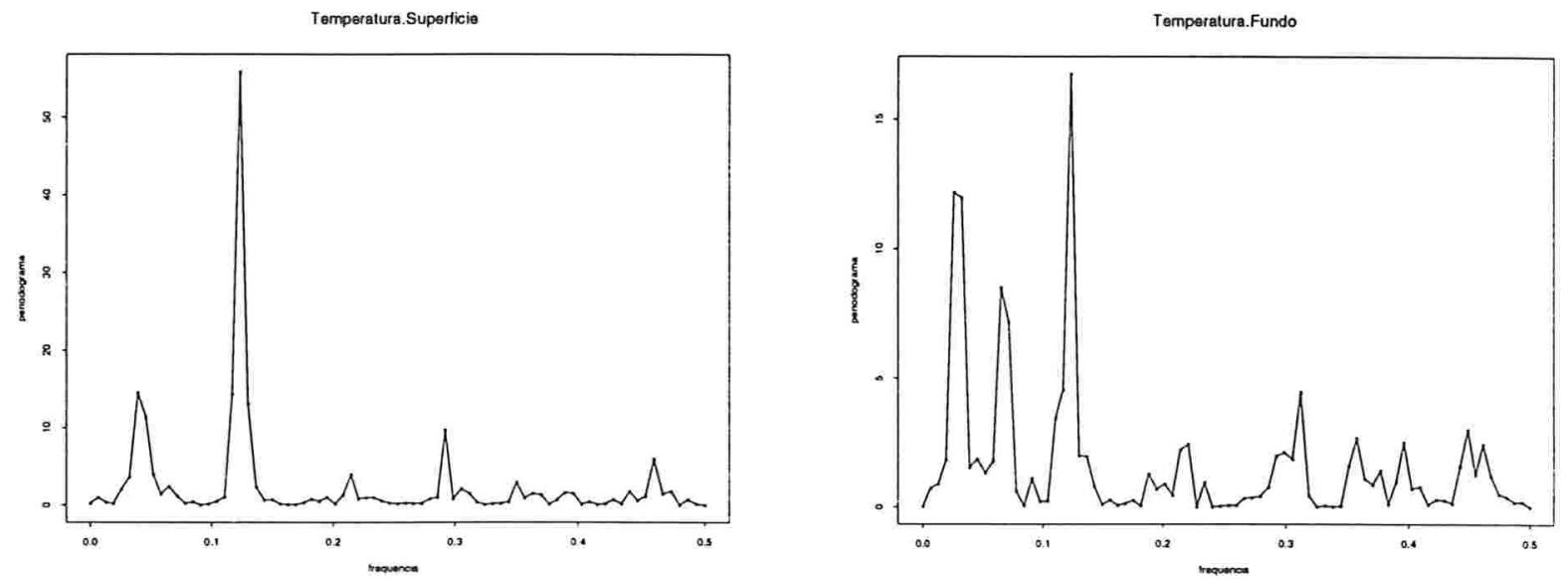

Figura 7.9: Periodograma das Diferenças Sazonais da Temperatura na Superfície e no Fundo 
Tabela 7.9. Algumas Variáveis do Teste das Somas Acumuladas

\begin{tabular}{||c|c|c|c|c||}
\hline \hline $\mathrm{j}$ & $J\left(\lambda_{i}\right)$ & $z_{i}$ & $c_{j}$ & $o_{j}$ \\
\hline \hline 1 & 1,31868 & 0,56437 & 0,56437 & 0,00892 \\
2 & 0,39398 & 1,26361 & 1,82799 & 0,02890 \\
3 & 0,11921 & 2,23947 & 4,06746 & 0,06432 \\
4 & 0,16771 & 1,94052 & 6,00799 & 0,09501 \\
5 & 0,30375 & 1,45678 & 7,46477 & 0,11804 \\
6 & 9,41099 & 0,10098 & 7,56575 & 0,11964 \\
7 & 6,09135 & 0,15200 & 7,71776 & 0,12204 \\
8 & 3,03547 & 0,28475 & 8,00251 & 0,12655 \\
9 & 0,79661 & 0,81329 & 8,81581 & 0,13941 \\
10 & 0,28504 & 1,50597 & 10,32171 & 0,16323 \\
11 & 0,16549 & 1,95195 & 12,27366 & 0,19409 \\
12 & 0,34404 & 1,36267 & 13,63633 & 0,21564 \\
13 & 8,61261 & 0,10984 & 13,74618 & 0,21738 \\
14 & 0,01620 & 4,13878 & 17,88497 & 0,28283 \\
15 & 0,61070 & 0,96981 & 18,85479 & 0,29817 \\
16 & 2,12962 & 0,38496 & 19,23975 & 0,30425 \\
17 & 0,30325 & 1,45804 & 20,69780 & 0,32731 \\
18 & 3,15055 & 0,27566 & 20,97346 & 0,33167 \\
19 & 3,33405 & 0,26231 & 21,23578 & 0,33582 \\
20 & 6,54891 & 0,14210 & 21,37788 & 0,33807 \\
21 & 1,18136 & 0,61327 & 21,99116 & 0,34777 \\
22 & 0,80053 & 0,81055 & 22,80172 & 0,36058 \\
23 & 6,93635 & 0,13467 & 22,93640 & 0,36272 \\
24 & 0,51150 & 1,08350 & 24,01990 & 0,37985 \\
25 & 0,71865 & 0,87191 & 24,89181 & 0,39364 \\
26 & 0,55443 & 1,03091 & 25,92273 & 0,40994 \\
27 & 1,18236 & 0,61289 & 26,53562 & 0,41963 \\
28 & 14,91833 & 0,06488 & 26,60050 & 0,42066 \\
29 & 0,37298 & 1,30321 & 27,90371 & 0,44127 \\
30 & 1,40758 & 0,53674 & 28,44046 & 0,44975 \\
\hline \hline
\end{tabular}


Tabela 7.9. Continuação

\begin{tabular}{||r|c|c|c|c||}
\hline \hline $\mathrm{j}$ & $J\left(\lambda_{i}\right)$ & $z_{i}$ & $c_{j}$ & $o_{j}$ \\
\hline \hline 31 & 0,16074 & 1,97699 & 30,41746 & 0,48102 \\
32 & 2,83296 & 0,30231 & 30,71977 & 0,48580 \\
33 & 1,78105 & 0,44562 & 31,16540 & 0,49285 \\
34 & 0,33531 & 1,38185 & 32,54725 & 0,51470 \\
35 & 48,66325 & 0,02034 & 32,56759 & 0,51502 \\
36 & 1,01588 & 0,68529 & 33,25289 & 0,52586 \\
37 & 46,27711 & 0,02137 & 33,27427 & 0,52620 \\
38 & 5,67005 & 0,16242 & 33,43670 & 0,52877 \\
39 & 2,32126 & 0,35823 & 33,79493 & 0,53443 \\
40 & 3,81226 & 0,23294 & 34,02787 & 0,53811 \\
41 & 0,68188 & 0,90281 & 34,93069 & 0,55239 \\
42 & 0,61338 & 0,96709 & 35,89778 & 0,56769 \\
43 & 1,86966 & 0,42843 & 36,32622 & 0,57446 \\
44 & 1,32569 & 0,56208 & 36,88830 & 0,58335 \\
45 & 4,89500 & 0,18589 & 37,07419 & 0,58629 \\
46 & 0,37780 & 1,29386 & 38,36806 & 0,60675 \\
47 & 1,12171 & 0,63736 & 39,00542 & 0,61683 \\
48 & 0,33820 & 1,37542 & 40,38085 & 0,63858 \\
49 & 0,82678 & 0,79276 & 41,17362 & 0,65112 \\
50 & 2,01356 & 0,40321 & 41,57683 & 0,65749 \\
51 & 3,20203 & 0,27178 & 41,84862 & 0,66179 \\
52 & 16,62373 & 0,05841 & 41,90703 & 0,66272 \\
53 & 9,73366 & 0,09779 & 42,00483 & 0,66426 \\
54 & 1,85302 & 0,43156 & 42,43639 & 0,67109 \\
55 & 0,33315 & 1,38670 & 43,82309 & 0,69302 \\
56 & 1,38445 & 0,54366 & 44,36676 & 0,70161 \\
57 & 1,53028 & 0,50287 & 44,86963 & 0,70957 \\
58 & 0,08388 & 2,55892 & 47,42856 & 0,75003 \\
59 & 6,29962 & 0,14733 & 47,57589 & 0,75236 \\
60 & 1,67947 & 0,46713 & 48,04303 & 0,75975 \\
\hline \hline
\end{tabular}


Tabela 7.9. Continuação

\begin{tabular}{||r|c|c|c|c||}
\hline \hline $\mathrm{j}$ & $J\left(\lambda_{i}\right)$ & $z_{i}$ & $c_{j}$ & $o_{j}$ \\
\hline \hline 61 & 0,60447 & 0,97619 & 49,01922 & 0,77519 \\
62 & 0,15287 & 2,02037 & 51,03960 & 0,80714 \\
63 & 0,54073 & 1,04708 & 52,08669 & 0,82370 \\
64 & 0,84316 & 0,78207 & 52,86876 & 0,83607 \\
65 & 0,55588 & 1,02924 & 53,89801 & 0,85234 \\
66 & 2,69938 & 0,31514 & 54,21315 & 0,85733 \\
67 & 0,86495 & 0,76831 & 54,98147 & 0,86948 \\
68 & 1,14496 & 0,62774 & 55,60921 & 0,87940 \\
69 & 0,19444 & 1,81528 & 57,42450 & 0,90811 \\
70 & 0,91692 & 0,73745 & 58,16195 & 0,91977 \\
71 & 2,42674 & 0,34505 & 58,50701 & 0,92523 \\
72 & 1,20268 & 0,60512 & 59,11213 & 0,93480 \\
73 & 3,52511 & 0,24972 & 59,36186 & 0,93875 \\
74 & 0,07854 & 2,61968 & 61,98155 & 0,98018 \\
75 & 3,74064 & 0,23691 & 62,21846 & 0,98392 \\
76 & 0,56720 & 1,01632 & 63,23479 & 1,00000 \\
\hline \hline
\end{tabular}

3. A estatística de Kolmogorov-Smirnov para comparar a distribuição dos $o_{j}$ forneceu um valor de 0,11398 correspondente a um nível descritivo de 0,28401 o que nos leva a aceitar a hipótese de igualdade das funções de densidade espectral.

\section{b) O Teste da Razão de Verossimilhança}

Os seguintes passos são utilizados na aplicação deste teste.

1. Os periodogramas e a razão dos periodogramas $J\left(\lambda_{i}\right)$ aparecem na Figura 7.9 e na segunda coluna da Tabela 7.9 , respectivamente.

2. O vetor das estatísticas $t_{i}=\ln \left(J\left(\lambda_{i}\right)\right), \quad i=1, \ldots, 76$, é dado por: $\mathbf{t}=\left[\begin{array}{llllllll}0,27660 & -0,93145 & -2,12684 & -1,78547 & -1,19153 & 2,24187 & 1,80687 & 1,11036\end{array}\right.$ $\begin{array}{llllllll}-0,22739 & -1,25510 & -1,79880 & -1,06699 & 2,15322 & -4,12271 & -0,49314 & 0,75594\end{array}-$ 
$1,193171,147571,204181,87929$ 0,16667-0,22247 1,93677-0,67039 -0,33036 $-0,589790,167512,70259-0,986220,34187-1,827931,041320,57720-1,09268$ $\begin{array}{llllllllll}3,88492 & 0,01575 & 3,83464 & 1,73519 & 0,84211 & 1,33822 & -0,38290 & -0,48876 & 0,62575\end{array}$ 0,28193 1,58821 -0,97337 0,11486-1,08409-0,19021 0,69990 1,16378 2,81083 $2,275590,61681-1,099150,325300,42545-2,47838$ 1,84048 0,51848 -0,50339 $\begin{array}{llllllll}-1,87811 & -0,61483 & -0,17059 & -0,58720 & 0,99302 & -0,14507 & 0,13537 & -1,63759\end{array}-$ $0,086730,886550,184551,25991-2,544071,31925-0,56703]$ '.

3. As estimativas dos parâmetros $\alpha, \beta$ e $\gamma$ do modelo logístico quadrático (5.6) forneceram os resultados

$$
\widehat{\alpha}=-0,53261, \quad \widehat{\beta}=6,84227, \quad \widehat{\gamma}=-12,96425 .
$$

4. O valor da estatística de razão de verossimilhança é 3,52675 fornecendo um nível descritivo de 0,31731 que nos leva, também, à aceitação da hipótese de igualdade das funções de densidade espectral.

A Tabela 7.10 apresenta um resumo da aplicação dos testes univariados para testar a igualdade das duas séries de temperatura.

Tabela 7.10. Resumo dos Testes Univariados

\begin{tabular}{||l|c||}
\hline \hline \multicolumn{1}{|c|}{ Teste } & Nivel Descritivo \\
\hline \hline Quenouille & 0,7954 \\
Mélard e Roy & 0,0220 \\
Coates e Diggle (SA) & 0,2840 \\
Coates e Diggle (RV) & 0,3173 \\
\hline \hline
\end{tabular}

Assim, a um nível de significância de 0,05 os testes de Quenouille e de Coates e Diggle aceitam a hipótese que as duas séries de temperatura, na superfície e no fundo, na estação de monitoramento BCG são geradas por processos com a mesma estrutura de dependência (porém com médias diferentes), mas o teste de Mélard e Roy rejeita esta hipótese, no entanto, a hipótese é aceita a um nível de significância de 0,01 . Uma estimativa das médias da temperatura são $\bar{x}_{\text {sup }}=30.6293$ e $\bar{x}_{\text {fundo }}=30.0654$. 


\subsection{Análise Multivariada das Séries}

Consideraremos aqui a comparação das séries bivariadas $\left\{X_{1}^{(1)}(t), X_{2}^{(1)}(t)\right\} \mathrm{e}$ $\left\{X_{1}^{(2)}(t), X_{2}^{(2)}(t)\right\}$ onde $\left\{X_{1}^{(1)}(t)\right\}$ e $\left\{X_{2}^{(1)}(t)\right\}$ representam a salinidade e a temperatura na superfície, $\left\{X_{1}^{(2)}(t)\right\}$ e $\left\{X_{2}^{(2)}(t)\right\}$ representam a salinidade e a temperatura no fundo, respectivamente. A comparação é feita utilizando os testes de Carmona e Wang com o objetivo de verificar se a série bivariada $\left\{X_{1}^{(1)}(t), X_{2}^{(1)}(t)\right\}$ tem o mesmo comportamento que $\left\{X_{1}^{(2)}(t), X_{2}^{(2)}(t)\right\}$.

Primeiramente fazemos um teste baseado em comparações pareadas (Johnson e Wichern (1998)) para verificar se os dois vetores de médias da salinidade e temperatura, na superfície e no fundo, respectivamente, são ou não iguais.

O procedimento é descrito a seguir:

1. Considerar as séries bivariadas na superfície e no fundo, respectivamente, na forma vetorial dada por

$$
\left[\begin{array}{l}
X_{1}^{(1)}(t) \\
X_{2}^{(1)}(t)
\end{array}\right] \text { e }\left[\begin{array}{l}
X_{1}^{(2)}(t) \\
X_{2}^{(2)}(t)
\end{array}\right] .
$$

2. Construir o vetor das diferenças $\mathbf{D}$ dado por

$$
\mathbf{D}=\left[\begin{array}{l}
X_{1}^{(1)}(t)-X_{1}^{(2)}(t) \\
X_{2}^{(1)}(t)-X_{2}^{(2)}(t)
\end{array}\right]
$$

Assume-se que este vetor tem distribuição $\mathbf{N}_{\mathbf{p}}\left(\mu_{\mathbf{D}}, \mathbf{S}_{\mathbf{D}}\right)$, com $p=2$.

3. Testar a hipótese $H_{0}: \mu_{\mathbf{D}}=\mathbf{0}$ contra a alternativa $H_{A}: \mu_{\mathbf{D}} \neq 0$. Rejeita-se a hipótese $H_{0}$ a um nível de significância $\alpha$, se o valor observado

$$
T^{2}=n \overline{\mathbf{D}}^{\prime} \mathbf{S}_{\mathbf{D}}^{-1} \overline{\mathbf{D}}>\frac{(n-1) p}{(n-p)} F_{p, n-p}(\alpha)
$$

onde $n$ representa o tamanho das séries, $\overline{\mathrm{D}}$ o vetor das médias das diferenças, $\mathrm{S}_{\mathrm{D}}$ a matriz de variâncias e covariâncias das diferenças e $F_{p, n-p}(\alpha)$ o $100 \alpha$ ésimo percentil da distribuição $\mathrm{F}$ com $p$ graus de liberdade no numerador e $n-p$ graus de liberdade no denominador.

A rejeição de $H_{0}$ implica que as médias das duas séries bivariadas são diferentes. 
Esse procedimento foi aplicado a um conjunto de 166 observações de cada uma das séries fornecendo os resultados:

$$
\begin{gathered}
\overline{\mathbf{D}}=\left[\begin{array}{c}
-2,95691 \\
0,56382
\end{array}\right], \quad \mathbf{S}_{\mathbf{D}}=\left[\begin{array}{cc}
18,1749 & -0,5258 \\
-0,5258 & 0,8464
\end{array}\right], \\
T^{2}=166[-2,956910,56382]\left[\begin{array}{cc}
18,1749 & -0,5258 \\
-0,5258 & 0,8464
\end{array}\right]\left[\begin{array}{c}
-2,95691 \\
0,56382
\end{array}\right]=125,539 \\
\mathrm{e} \\
\frac{165 \times 2}{164} F_{2,164}(0,95)=6,139 .
\end{gathered}
$$

Como $T^{2}=125,5>6,139$, rejeitamos a hipótese $H_{0}$ e concluímos que os vetores média das duas séries bivariadas em diferentes profundidades de água são diferentes.

Prosseguimos a análise com o objetivo de verificar se a estrutura de dependência das séries bivariadas é a mesma.

A Figura 7.10 representa os módulos das entradas da estimativa da matriz de densidade espectral suavizada de tamanho $4 \times 4, f_{\mathbf{X}}\left(\lambda_{j}\right)$, das séries bivariadas $\left\{X_{1}^{(1)}(t), X_{2}^{(1)}(t)\right\}$ e $\left\{X_{1}^{(2)}(t), X_{2}^{(2)}(t)\right\}$. Na suavização desta matriz, usamos $\mathrm{L}=5$ valores de periodogramas.

\subsubsection{Testes de Carmona e Wang}

\section{a) Comparação das Séries no Caso de Independência}

Usando a estatística traço, dada por (5.14) e assumindo independência entre as séries $\left\{X_{1}^{(1)}(t), X_{2}^{(1)}(t)\right\}$ e $\left\{X_{1}^{(2)}(t), X_{2}^{(2)}(t)\right\}$, obtemos os seguintes resultados:

1. O vetor de estatísticas traço, $\mathbf{T}$, de tamanho $(1 \times 14)$ e que corresponde aos traços dos produtos das matrizes de densidade espectral estimada das séries bivariadas $\left\{X_{1}^{(1)}(t), X_{2}^{(1)}(t)\right\}$ e $\left\{X_{1}^{(2)}(t), X_{2}^{(2)}(t)\right\}$ é dado por $\mathbf{T}=[1,8178,3,9121,1,4572,4,5471,2,0761,2,3065,4,20972,5,8119,3,0606$, $2,9770,4,4579,2,9175,2,6182,5,5006]$. 

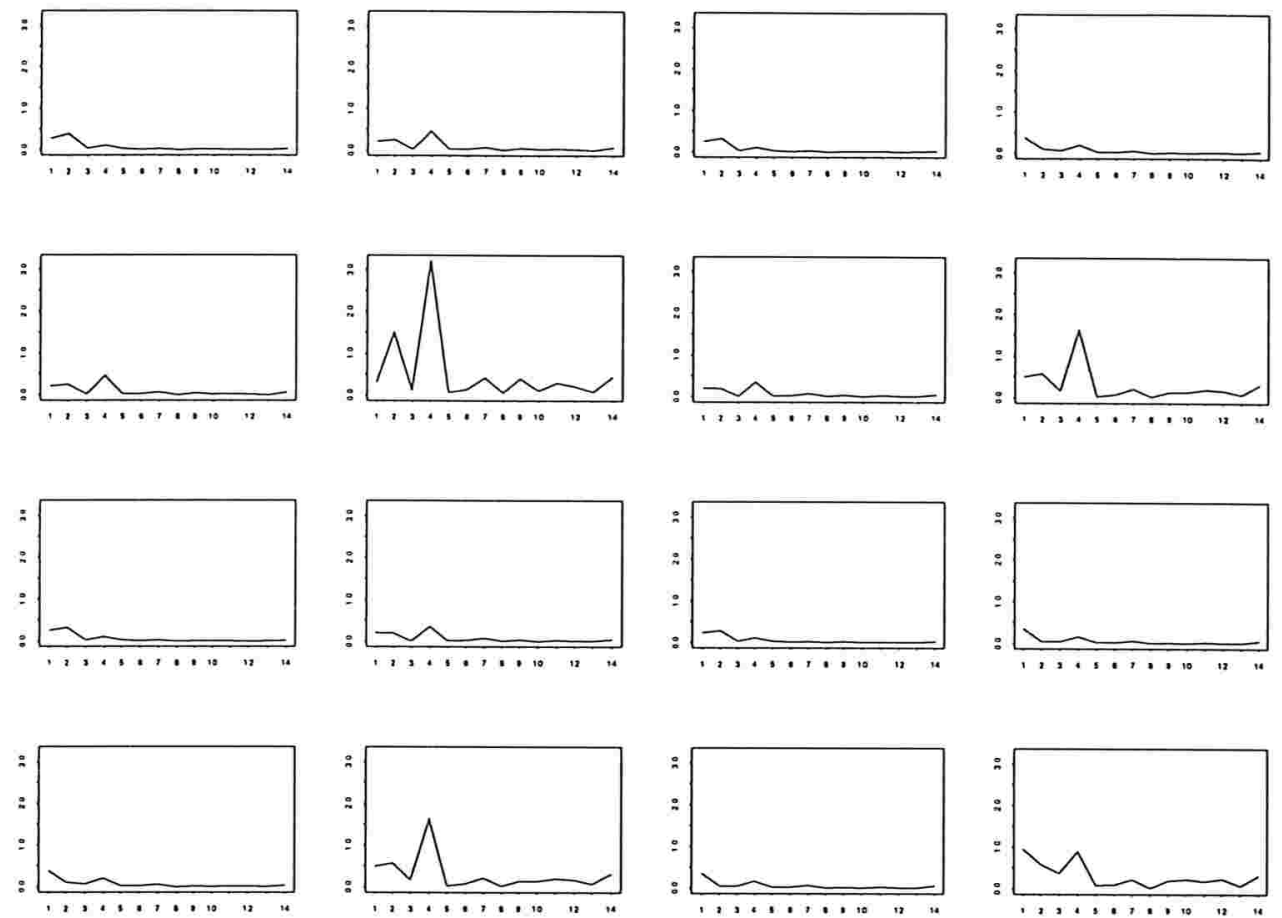

Figura 7.10: Módulo das Entradas da Matriz de Densidade Espectral Estimada

2. O vetor de estatísticas traço, $\mathbf{T}^{*}$, de tamanho (1x14), formado pelos traços do tipo Monte Carlo é dado por

$\mathbf{T}^{*}=[3,6611,4,3280,3,3670,2,0479,1,8812,2,1415,2,1605,1,8679,3,1620$, $2,4345,3,9932,4,0515,2,8674,2,7795]$.

3. O teste de Kolmogorov-Smirnov(Apêndice C) aplicado às duas amostras $\mathrm{T}$ e $\mathrm{T}^{*}$, forneceu um nível descritivo de 0,54074 , que nos leva à aceitação da hipótese de igualdade das matrizes de densidade espectrais das séries bivariadas.

\section{b) Comparação das Séries no Caso de Dependência}

Neste caso aplicamos os dois procedimentos sugeridos.

1. Quando assumimos dependência entre as séries $\left\{X_{1}^{(1)}(t), X_{2}^{(1)}(t)\right\}$ e $\left\{X_{1}^{(2)}(t), X_{2}^{(2)}(t)\right\}$ e utilizando o primeiro procedimento descrito na seção 5.2.2, geramos 1000 
réplicas bootstrap que forneceram as estatísticas $T_{K S}^{b}, b=1, \ldots, 1000$ cujo histograma está representado na Figura 7.11. $\mathrm{O}$ valor observado $T_{K S_{o b s}}=$ 0,42857 junto com o histograma fornecem um nível descritivo de 0,064 que nos leva à aceitação da hipótese de igualdade das matrizes de densidade espectrais das séries bivariadas.

Histograma

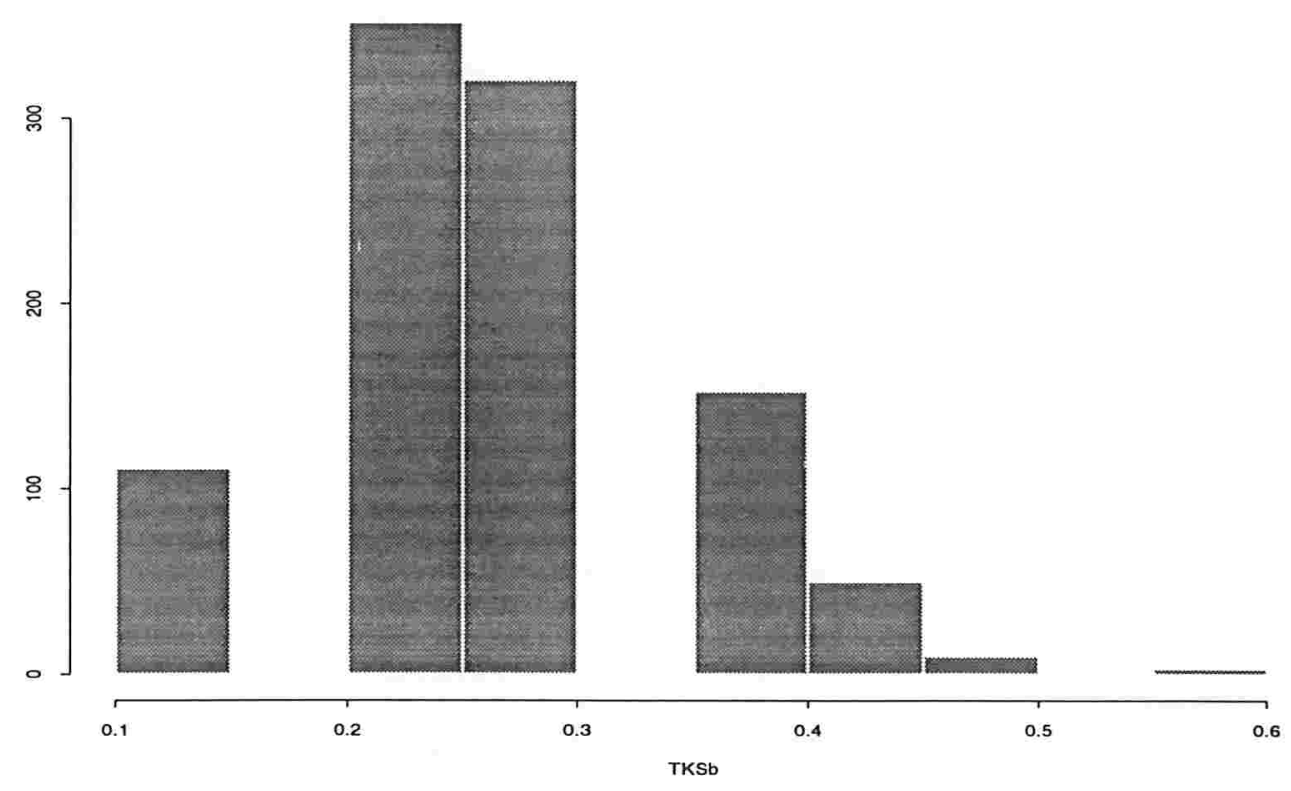

Figura 7.11: Histograma das estatísticas tipo bootstrap

2. Utilizando o segundo procedimento descrito na seção 5.2 .2 , obtemos o vetor de traços, do tipo Monte Carlo, dado por

$\mathbf{T}^{*}=[2,878252,2,820556,2,697550,3,401028,1,413149,1,511414,1,179629$, 1,891015, 4,085084, 2,675872, 1,771545, 2,644145, 4,395269, 2,038589].

$\mathrm{O}$ teste de Kolmogorov-Smirnov aplicado às duas amostras $\mathbf{T} \mathrm{e} \mathbf{T}^{*}$, forneceu um nível descritivo de 0,1106 , que também nos leva à aceitação da hipótese de igualdade das matrizes de densidade espectrais das séries bivariadas. 
Em resumo, a suposição de independência ou não, não interfere no resultado da aplicação do teste de Carmona e Wang. Assim, a um nível de significância de $5 \%$, podemos concluir que apesar das séries bivariadas de salinidade e temperatura apresentarem médias diferentes na superfície e fundo da lagoa, elas têm a mesma estrutura de dependência de segunda ordem.

\subsection{Conclusões}

Em nossa análise dos dados de temperatura e salinidade verificamos que:

1. Foi detectado, ao nível de 0,05, uma diferença entre as médias de salinidade na superfície e no fundo da lagoa. A ocorrência de uma salinidade média maior no fundo da lagoa já era esperada. Também foi detectada uma diferença entre as médias de temperatura na superfície e no fundo da lagoa, entretanto, neste caso a temperatura média na superfície é maior do que no fundo, o que também era esperado.

2. Os testes univariados, quando utilizados para verificar se salinidade na superfície e fundo da lagoa têm a mesma estrutura de dependência, forneceram o mesmo resultado a um nível de significância de $5 \%$, isto é, todos aceitaram que as duas séries, corrigidas pela média, têm o mesmo comportamento. $\mathrm{O}$ mesmo acontece às duas séries de temperatura, se considerarmos um nível de significância de 0,01 .

3. Um teste bivariado de comparação de médias, também constatou, ao nível de significância de 0,05 , que as séries na superfície e no fundo têm médias diferentes.

4. Como mencionado na seção anterior, quando utilizamos técnicas multivariadas, para verificar se a série bivariada de salinidade e temperatura na superfície da água têm a mesma estrutura de dependência que a série salinidade e temperatura no fundo da água, verificamos que a suposição de dependência, ou não, entre as séries bivariadas, não altera o resultado de que as duas séries, corrigidas pela média, têm o mesmo comportamento de segunda ordem. 
5. Como conclusão final podemos sugerir que se tome uma única medida (na superfície) das séries de salinidade e temperatura e se for de interesse obter uma amostra de cada uma delas no fundo, basta fazer a respectiva correção da média. 


\section{Referências}

Anderson, T. (1971). The Statistical Analysis of Time Series. New York: John Wiley.

Billingsley, P. (1968). Convergence of Probability Measures. New York: John Wiley.

Bloomfield, D. (1976). Fourier Analysis of Time Series: An Introduction. New York: John Wiley.

Botero, L. and E. Mancera (1996). Síntesis de los cambios de origen antrópico ocurridos en los últimos 40 años en la Ciénaga Grande de Santa Marta. Revista Académica Colombiana de Ciencias Exactas, Físicas y Naturales. $20(78), 465-473$.

Box, G. and D. Cox (1964). An analysis of transformations. Journal of Royal Statist. Soc. Ser. B 26, 211-243.

Box, G.E, Jenkins, G.M. and Reinsel, G.C. (1994). Time Series Analysis: Forescasting and Control (3 ed.). San Francisco: Holden-Day.

Brillinger, D. (1981). Time Series: Data Analysis and Theory (2 ed.). San Francisco: Holden-Day.

Brockwell, P. and R. Davis (1991). Time Series: Theory and Methods (2 ed.). New York: Springer Verlag.

Carmona, R. and A. Wang (1996). Comparison tests for the spectra of dependent multivariate time series. Stochastic Modelling in Physical Oceanography 39(1), 69-88.

Coates, D. and P. Diggle (1986). Tests for comparing two estimated spectral densities. Journal of Time Series Analysis 7(1), 7-20. 
Conover, W. (1980). Practical Nonparametric Statistics (2 ed.). New York: John Wiley.

Daniels, H. (1956). The approximate distribution of serial correlation coefficient. Biometrika 43, 169-185.

Darling, D. (1957). The Kolmogorov-Smirnov, Cramér-Von Mises Tests. The Annals of Mathematical Statistics 28(1), 823-838.

Fuller, W. (1976). Introduction to Statistical Time Series. New York: John Wiley.

Gibbons, J. (1985). Nonparametric Statistical Inference (2 ed.). New York: Marcel Dekker.

Goodman, N. (1963). Statistical Analysis based upon a certain multivariate complex Gaussian distribution (an Introduction). The Annals of Mathematics Statistics 34, 152-177.

Jenkins, G. (1956). Tests of hipotheses in the linear autoregressive model II. Biometrika 43, 186-199.

Johnson, R. and D. Wichern (1998). Applied Multivariate Statistical Analysis (4 ed.). New Jersey: Prentice-Hall, Inc.

Kabe, D. (1966). Complex analogues of some classical non-central multivariate distributions. Australian Journal of Statistics 8, 99-103.

Leonov, V. and A. Shiryaev (1959). On a method of calculation of semiinvariants. Theory of Probability and its Applications IV (3), 319-328.

Loéve, M. (1978). Probability Theory II. New York: Springer-Verlag.

Miller, K. (1968). Moments of complex Gaussian processes. Proc. IEEE 56, 83-84.

Mélard, G. and R. Roy (1984). Sur un test d'egalité des autocovariances de deux séries chronologiques. La Revue Canadienne de Statistique 12(4), 333-342.

Morettin, P. (1979). Análise Harmônica de Processos Estocásticos. Rio de Janeiro: IMPA.

Priestley, M. (1981). Spectral Analysis and Time Series, Volume 1,2. New York: Academic Press. 
Quenouille, M. (1958). The comparison of correlations in time-series. Journal Royal Statist. Soc. Ser. B 20(1), 158-164.

Robinson, P. (1977). Estimating variances and covariances of sample autocorrelations and autocovariances. Australian Journal of Statistics (19), 236-240.

Smirnov, N. (1939a). On the estimation of the discrepancy between empirical curves of distribution for two independent samples. Bull. Math. University of Moscow 2(2), 3-14.

Smirnov, N. (1939b). On the estimation of the discrepancy between empirical curves of distribution for two independent samples. Bulletin of Mathematics Univ. Moscow 2, 3-14.

Srivastava, M. (1965). On the complex wishart distribution. The Annals of Mathematics Statistics 36, 313-315.

Wei, W. (1990). Time Series Analysis: Univariate and Multivariate Methods. California: Addison-Wesley. 


\section{Apêndice A}

\section{Séries Salinidade e Temperatura}

Tabela A.1. Séries de Salinidade(\%) e Temperatura $\left({ }^{\circ} C\right)$ da água em BCG.

(Fonte: INVEMAR - Santa Marta - Colômbia).

\begin{tabular}{||l||c||c||c||c||}
\hline \hline Data & $\begin{array}{c}\text { Salinidade } \\
\text { na superfície }\end{array}$ & $\begin{array}{c}\text { Salinidade } \\
\text { no fundo }\end{array}$ & $\begin{array}{c}\text { Temperatura } \\
\text { na superfície }\end{array}$ & $\begin{array}{c}\text { Temperatura } \\
\text { no fundo }\end{array}$ \\
\hline \hline Mar-82 & 15,0 & 15,4 & 31,0 & 30,0 \\
Abr-82 & 22,1 & 23,7 & 31,0 & 30,0 \\
Mai-82 & 20,7 & 20,9 & 32,0 & 32,0 \\
Jun-82 & 11,5 & 13,4 & 32,2 & 31,3 \\
Jul-82 & 13,3 & 14,8 & 31,0 & 30,2 \\
Ago-82 & 17,4 & 18,0 & 31,5 & 31,2 \\
Set-82 & 15,4 & 20,0 & 30,1 & 29,5 \\
Out-82 & 12,9 & 15,2 & 30,5 & 31,0 \\
Nov-82 & 11,6 & 12,7 & 30,0 & 28,7 \\
Dez-82 & 19,3 & 18,1 & 27,2 & 26,5 \\
Jan-83 & 25,0 & 25,5 & 29,0 & 28,5 \\
Fev-83 & 27,1 & 27,4 & 27,5 & 27,5 \\
Mar-83 & 25,7 & 26,7 & 29,5 & 29,3 \\
Abr-83 & 23,9 & 27,3 & 31,2 & 29,3 \\
Mai-83 & 24,9 & 25,5 & 31,5 & 30,5 \\
Jun-83 & 21,9 & 23,2 & 32,0 & 30,6 \\
\hline \hline
\end{tabular}


Tabela A.1. Continuação

\begin{tabular}{||l||c||c||c||c||}
\hline \hline Data & $\begin{array}{c}\text { Salinidade } \\
\text { na superfície }\end{array}$ & $\begin{array}{c}\text { Salinidade } \\
\text { no fundo }\end{array}$ & $\begin{array}{c}\text { Temperatura } \\
\text { na superfície }\end{array}$ & $\begin{array}{c}\text { Temperatura } \\
\text { no fundo }\end{array}$ \\
\hline \hline Jul-83 & 17,8 & 23,1 & 31,6 & 30,5 \\
Ago-83 & 16,5 & 23,6 & 31,2 & 31,3 \\
Set-83 & 17,1 & 23,5 & 30,7 & 30,8 \\
Out-83 & 16,9 & 22,2 & 30,7 & 30,8 \\
Nov-83 & 15,1 & 21,2 & 30,7 & 30,8 \\
Dez-83 & 15,5 & 22,7 & 30,0 & 30,2 \\
Jan-84 & 19,3 & 24,4 & 29,8 & 30,1 \\
Fev-84 & 22,8 & 25,2 & 29,9 & 29,3 \\
Mar-84 & 25,0 & 25,1 & 30,3 & 29,5 \\
Abr-84 & 25,4 & 25,3 & 30,8 & 30,0 \\
Mai-84 & 25,8 & 25,1 & 31,6 & 29,6 \\
Jun-84 & 24,3 & 25,0 & 32,2 & 30,2 \\
Jul-84 & 23,2 & 25,1 & 31,6 & 29,4 \\
Ago-84 & 24,1 & 25,8 & 31,2 & 30,4 \\
Set-84 & 21,8 & 24,2 & 30,2 & 31,0 \\
Out-84 & 19,8 & 23,0 & 29,1 & 30,4 \\
Nov-84 & 19,7 & 21,3 & 29,7 & 31,1 \\
Dez-84 & 19,0 & 23,1 & 28,7 & 30,7 \\
Jan-85 & 19,2 & 23,9 & 29,5 & 31,0 \\
Fev-85 & 19,1 & 25,9 & 29,7 & 30,3 \\
Mar-85 & 20,6 & 26,1 & 29,6 & 30,8 \\
Abr-85 & 23,4 & 26,8 & 31,3 & 30,4 \\
Mai-85 & 24,1 & 27,5 & 31,6 & 29,4 \\
Jun-85 & 25,6 & 29,4 & 32,0 & $29,9,9$ \\
Jul-85 & 25,7 & 29,9 & 31,7 & 29,2 \\
Ago-85 & 27,2 & 32,5 & 31,2 & 28,8 \\
Set-85 & 23,2 & 25,0 & 30,9 & \\
Out-85 & 24,3 & 20,8 & 31,3 \\
Nov-85 & 22,2 & 20,2 & \\
Dez-85 & 18,8 & & \\
\hline \hline
\end{tabular}


Tabela A.1. Continuação

\begin{tabular}{|c|c|c|c|c|}
\hline Data & $\begin{array}{c}\text { Salinidade } \\
\text { na superfície }\end{array}$ & $\begin{array}{c}\text { Salinidade } \\
\text { no fundo }\end{array}$ & $\begin{array}{l}\text { Temperatura } \\
\text { na superfície }\end{array}$ & $\begin{array}{c}\text { Temperatura } \\
\text { no fundo }\end{array}$ \\
\hline Jan-86 & 22,5 & 21,7 & 29,2 & 29,6 \\
\hline Fev-86 & 23,1 & 29,8 & 29,7 & 29,8 \\
\hline Mar-86 & 23,3 & 29,5 & 29,5 & 29,7 \\
\hline Abr- 86 & 23,6 & 29,3 & 30,5 & 31,3 \\
\hline Mai-86 & 24,6 & 28,2 & 31,8 & 30,6 \\
\hline Jun-86 & 24,4 & 25,9 & 33,3 & 30,7 \\
\hline Jul-86 & 25,4 & 24,8 & 32,7 & 30,7 \\
\hline Ago-86 & 27,4 & 25,5 & 31,2 & 29,5 \\
\hline Set-86 & 30,2 & 24,7 & 29,5 & 29,2 \\
\hline Out-86 & 16,1 & 22,0 & 25,5 & 28,1 \\
\hline Nov-86 & 23,4 & 19,2 & 28,0 & 29,3 \\
\hline Dez-86 & 24,8 & 25,9 & 28,0 & 27,5 \\
\hline Jan-87 & 19,0 & 19,9 & 29,0 & 29,2 \\
\hline Fev-87 & 28,9 & 29,6 & 31,0 & 31,0 \\
\hline Mar-87 & 32,1 & 32,7 & 28,8 & 29,6 \\
\hline Abr- 87 & 34,8 & 31,8 & 32,0 & 31,7 \\
\hline Mai-87 & 30,7 & 32,6 & 32,2 & 32,5 \\
\hline Jun-87 & 22,2 & 24,0 & 32,7 & 31,5 \\
\hline Jul-87 & 21,8 & 21,9 & 32,2 & 31,2 \\
\hline Ago-87 & 19,0 & 26,0 & 31,5 & 30,3 \\
\hline Set- 87 & 18,0 & 29,5 & 31,5 & 31,2 \\
\hline Out-87 & 15,0 & 21,5 & 34,0 & 32,0 \\
\hline Nov-87 & 10,4 & 12,0 & 33,5 & 32,2 \\
\hline Dez-87 & 14,3 & 16,2 & 30,7 & 31,1 \\
\hline Jan- 88 & 20,6 & 15,5 & 28,0 & 28,5 \\
\hline Fev-88 & 24,6 & 24,7 & 29,0 & 28,6 \\
\hline Mar-88 & 28,0 & 28,0 & 28,0 & 28,0 \\
\hline Abr-88 & 32,4 & 32,5 & 29,2 & 29,0 \\
\hline Mai-88 & 34,7 & 34,8 & 31,0 & 29,7 \\
\hline Jun-88 & 29,0 & 29,9 & 33,5 & 32,0 \\
\hline
\end{tabular}


Tabela A.1. Continuação

\begin{tabular}{|c|c|c|c|c|}
\hline Data & $\begin{array}{c}\text { Salinidade } \\
\text { na superfície }\end{array}$ & $\begin{array}{c}\text { Salinidade } \\
\text { no fundo }\end{array}$ & $\begin{array}{l}\text { Temperatura } \\
\text { na superfície }\end{array}$ & $\begin{array}{c}\text { Temperatura } \\
\text { no fundo }\end{array}$ \\
\hline Jul-88 & 20,4 & 26,3 & 33,5 & 33,0 \\
\hline Ago-88 & 17,3 & 23,9 & 28,5 & 28,5 \\
\hline Set- 88 & 12,1 & 15,0 & 31,0 & 31,0 \\
\hline Out- 88 & 5,7 & 5,7 & 29,5 & 29,3 \\
\hline Nov-88 & 1,2 & 4,6 & 29,3 & 29,7 \\
\hline Dez-88 & 0,8 & 0,8 & 29,5 & 29,5 \\
\hline Jan-89 & 0,7 & 0,8 & 28,0 & 27,0 \\
\hline Fev-89 & 6,2 & 7,8 & 29,0 & 27,0 \\
\hline Mar-89 & 15,4 & 19,3 & 30,5 & 28,0 \\
\hline Abr- 89 & 21,9 & 21,9 & 31,5 & 29,7 \\
\hline Mai-89 & 27,2 & 27,2 & 31,5 & 30,5 \\
\hline Jun-89 & 22,4 & 24,3 & 31,0 & 31,0 \\
\hline Jul-89 & 22,7 & 23,4 & 29,2 & 29,2 \\
\hline Ago-89 & 23,1 & 23,0 & 31,0 & 31,0 \\
\hline Set-89 & 20,0 & 20,1 & 30,0 & 30,0 \\
\hline Out-89 & 15,1 & 16,1 & 32,8 & 31,5 \\
\hline Nov-89 & 11,9 & 14,0 & 32,5 & 30,6 \\
\hline Dez-89 & 9,5 & 13,0 & 31,0 & 29,8 \\
\hline Jan-90 & 13,5 & 13,5 & 29,0 & 28,5 \\
\hline Fev-90 & 19,3 & 30,4 & 29,0 & 29,0 \\
\hline Mar-90 & 26,2 & 26,2 & 28,0 & 28,0 \\
\hline Abr-90 & 32,6 & 32,6 & 29,0 & 29,0 \\
\hline Mai-90 & 30,3 & 30,5 & 31,5 & 31,5 \\
\hline Jun-90 & 24,8 & 25,0 & 33,0 & 32,3 \\
\hline Jul-90 & 23,1 & 26,5 & 32,0 & 31,0 \\
\hline Ago-90 & 23,2 & 23,1 & 32,8 & 32,0 \\
\hline Set-90 & 20,9 & 24,5 & 31,0 & 30,8 \\
\hline Out-90 & 14,9 & 15,0 & 30,0 & 29,8 \\
\hline Nov-90 & 9,3 & 16,1 & 31,5 & 31,0 \\
\hline Dez-90 & 10,0 & 15,5 & 30,0 & 30,0 \\
\hline
\end{tabular}


Tabela A.1. Continuação

\begin{tabular}{|c|c|c|c|c|}
\hline Data & $\begin{array}{c}\text { Salinidade } \\
\text { na superfície }\end{array}$ & $\begin{array}{c}\text { Salinidade } \\
\text { no fundo }\end{array}$ & $\begin{array}{l}\text { Temperatura } \\
\text { na superfície }\end{array}$ & $\begin{array}{c}\text { Temperatura } \\
\text { no fundo }\end{array}$ \\
\hline Jan-91 & 14,6 & 17,5 & 29,5 & 29,0 \\
\hline Fev-91 & 17,9 & 17,9 & 28,0 & 28,0 \\
\hline Mar-91 & 28,0 & 27,9 & 28,3 & 28,1 \\
\hline Abr-91 & 31,2 & 31,2 & 31,0 & 30,5 \\
\hline Mai-91 & 32,8 & 32,8 & 30,8 & 30,8 \\
\hline Jun-91 & 31,0 & 32,0 & 31,0 & 32,0 \\
\hline Jul-91 & 25,9 & 28,0 & 30,6 & 30,4 \\
\hline Ago-91 & 24,3 & 26,0 & 31,2 & 29,9 \\
\hline Set-91 & 18,3 & 26,1 & 30,4 & 29,8 \\
\hline Out-91 & 16,8 & 24,7 & 32,3 & 30,0 \\
\hline Nov-91 & 11,9 & 24,1 & 31,5 & 30,2 \\
\hline Dez-91 & 13,5 & 24,1 & 31,5 & 30,3 \\
\hline Jan-92 & 20,5 & 26,5 & 29,9 & 29,1 \\
\hline Fev-92 & 26,5 & 27,9 & 28,9 & 28,2 \\
\hline Mar-92 & 32,6 & 32,8 & 28,5 & 28,0 \\
\hline Abr-92 & 36,6 & 36,9 & 30,0 & 30,0 \\
\hline Mai-92 & 40,1 & 40,5 & 30,2 & 30,0 \\
\hline Jun-92 & 37,9 & 37,9 & 33,0 & 33,0 \\
\hline Jul-92 & 23,3 & 25,4 & 32,0 & 31,0 \\
\hline Ago-92 & 22,4 & 22,5 & 31,0 & 31,0 \\
\hline Set-92 & 21,0 & 25,2 & 31,5 & 31,3 \\
\hline Out-92 & 17,7 & 29,7 & 33,5 & 32,5 \\
\hline Nov-92 & 14,1 & 26,1 & 30,5 & 29,5 \\
\hline Dez-92 & 16,3 & 25,5 & 31,0 & 31,0 \\
\hline Jan-93 & 22,7 & 32,3 & 30,6 & 29,4 \\
\hline Fev-93 & 30,2 & 31,1 & 28,0 & 29,0 \\
\hline Mar-93 & 30,8 & 30,6 & 30,0 & 30,6 \\
\hline Abr-93 & 33,6 & 32,2 & 32,1 & 30,8 \\
\hline Mai-93 & 25,8 & 31,8 & 31,9 & 32,1 \\
\hline Jun-93 & 18,6 & 27,7 & 31,9 & 31,6 \\
\hline
\end{tabular}


Tabela A.1. Continuação

\begin{tabular}{|c|c|c|c|c|}
\hline Data & $\begin{array}{c}\text { Salinidade } \\
\text { na superfície }\end{array}$ & $\begin{array}{c}\text { Salinidade } \\
\text { no fundo }\end{array}$ & $\begin{array}{l}\text { Temperatura } \\
\text { na superfície }\end{array}$ & $\begin{array}{c}\text { Temperatura } \\
\text { no fundo }\end{array}$ \\
\hline Jul-93 & 35,3 & 36,3 & 31,0 & 31,0 \\
\hline Ago-93 & 18,0 & 18,0 & 31,1 & 30,6 \\
\hline Set-93 & 18,7 & 26,3 & 29,7 & 28,7 \\
\hline Out-93 & 11,5 & 12,0 & 32,6 & 31,8 \\
\hline Nov-93 & 27,8 & 27,8 & 31,0 & 30,5 \\
\hline Dez-93 & 13,4 & 26,5 & 32,8 & 30,5 \\
\hline Jan-94 & 27,4 & 28,0 & 29,8 & 29,3 \\
\hline Fev-94 & 28,8 & 32,8 & 29,0 & 29,4 \\
\hline Mar-94 & 33,5 & 33,8 & 28,6 & 28,8 \\
\hline Abr-94 & 36,7 & 38,5 & 30,5 & 29,6 \\
\hline Mai-94 & 31,4 & 31,6 & 31,8 & 30,7 \\
\hline Jun-94 & 24,8 & 38,1 & 32,5 & 31,9 \\
\hline Jul-94 & 21,4 & 36,5 & 31,6 & 30,0 \\
\hline Ago-94 & 32,4 & 33,0 & 30,7 & 30,1 \\
\hline Set-94 & 27,3 & 38,7 & 33,3 & 30,7 \\
\hline Out-94 & 19,3 & 39,1 & 31,6 & 29,3 \\
\hline Nov-94 & 18,3 & 35,2 & 30,6 & 29,8 \\
\hline Dez-94 & 25,1 & 40,1 & 31,3 & 30,4 \\
\hline Jan-95 & 7,7 & 8,1 & 30,2 & 28,9 \\
\hline Fev-95 & 28,6 & 28,6 & 28,4 & 27,3 \\
\hline Mar-95 & 33,1 & 33,2 & 28,1 & 27,8 \\
\hline Abr-95 & 38,1 & 38,9 & 29,3 & 29,2 \\
\hline Mai-95 & 36,1 & 39,5 & 32,1 & 31,6 \\
\hline Jun-95 & 35,9 & 44,3 & 33,4 & 32,0 \\
\hline Jul-95 & 32,8 & 41,0 & 31,5 & 31,1 \\
\hline Ago-95 & 16,5 & 22,1 & 29,8 & 30,0 \\
\hline Set-95 & 6,8 & 11,4 & 31,4 & 30,3 \\
\hline Out-95 & 5,1 & 7,5 & 28,5 & 27,8 \\
\hline Nov-95 & 3,4 & 3,5 & 30,7 & 30,2 \\
\hline Dez-95 & 3,6 & 3,7 & 30,2 & 29,0 \\
\hline
\end{tabular}




\section{Apêndice B}

\section{Resultados das Simulações para Séries Univariadas}

Tabela B.1. Poder Estimado para os Testes Univariados de Comparação de Modelos AR(1)

\begin{tabular}{|c|c|c|c|c|c|c|c|c|c|c|c|c|}
\hline \multirow{2}{*}{$\begin{array}{l}\text { Teste } \\
\phi_{1}-\phi_{1} / \mathrm{T}\end{array}$} & \multicolumn{3}{|c|}{ Quenouille, $\mathrm{J}=7$} & \multicolumn{3}{|c|}{ Mélard\&Roy, $\mathrm{J}=8$} & \multicolumn{3}{|c|}{ Coat\&Digg(SA) } & \multicolumn{3}{|c|}{ Coat\&Digg(RV) } \\
\hline & 100 & 400 & 800 & 100 & 400 & 800 & 100 & 400 & 800 & 100 & 400 & 800 \\
\hline $0,2-0,2$ & 0,034 & 0,042 & 0,051 & 0,012 & 0,018 & 0,033 & 0,066 & 0,062 & 0,058 & 0,062 & 0,073 & 0,058 \\
\hline 0,3 & 0,034 & 0,130 & 0,253 & 0,006 & 0,063 & 0,194 & 0,069 & 0,153 & 0,258 & 0,092 & 0,150 & 0,268 \\
\hline 0,4 & 0,098 & 0,531 & 0,877 & 0,025 & 0,388 & 0,833 & 0,147 & 0,417 & 0,752 & 0,169 & 0,501 & 0,836 \\
\hline 0,5 & 0,235 & 0,910 & 1,0 & 0,048 & 0,839 & 0,999 & 0,238 & 0,754 & 0,973 & 0,303 & 0,878 & 0,996 \\
\hline 0,6 & 0,522 & 1,0 & 1,0 & 0,134 & 0,995 & 1,0 & 0,354 & 0,952 & 0,999 & 0,523 & 0,992 & 1,0 \\
\hline 0,7 & 0,809 & 1,0 & 1,0 & 0,270 & 1,0 & 1,0 & 0,503 & 0,991 & 1,0 & 0,734 & 1,0 & 1,0 \\
\hline 0,8 & 0,947 & 1,0 & 1,0 & 0,438 & 1,0 & 1,0 & 0,626 & 1,0 & 1,0 & 0,915 & 1,0 & 1,0 \\
\hline
\end{tabular}


Tabela B.2. Poder Estimado para os Testes Univariados de Comparação de Modelos MA(1)

\begin{tabular}{||c||ccc||ccc||ccc||ccc||}
\hline \hline \multicolumn{1}{||l||}{ Teste } & \multicolumn{2}{|c||}{ Quenouille, J=7 } & \multicolumn{3}{|c||}{ Mélard\&Roy,J=8 } & \multicolumn{3}{|c||}{ Coat\&Digg(SA) } & \multicolumn{3}{||}{ Coat\&Digg(RV) } \\
\hline \hline$\theta_{1}-\theta_{1} / \mathrm{T}$ & 100 & 400 & 800 & 100 & 400 & 800 & 100 & 400 & 800 & 100 & 400 & 800 \\
\hline \hline $0,2-0,2$ & 0,026 & 0,038 & 0,043 & 0,004 & 0,019 & 0,033 & 0,072 & 0,053 & 0,059 & 0,084 & 0,068 & 0,059 \\
0,3 & 0,026 & 0,107 & 0,229 & 0,002 & 0,071 & 0,178 & 0,090 & 0,164 & 0,266 & 0,081 & 0,174 & 0,266 \\
0,4 & 0,084 & 0,491 & 0,849 & 0,018 & 0,372 & 0,791 & 0,153 & 0,497 & 0,762 & 0,175 & 0,532 & 0,762 \\
0,5 & 0,180 & 0,895 & 0,999 & 0,045 & 0,829 & 0,997 & 0,288 & 0,841 & 0,987 & 0,318 & 0,883 & 0,987 \\
0,6 & 0,352 & 0,994 & 1,0 & 0,110 & 0,983 & 1,0 & 0,511 & 0,980 & 1,0 & 0,525 & 0,990 & 1,0 \\
0,7 & 0,528 & 1,0 & 1,0 & 0,176 & 1,0 & 1,0 & 0,689 & 1,0 & 1,0 & 0,709 & 1,0 & 1,0 \\
0,8 & 0,712 & 1,0 & 1,0 & 0,329 & 1,0 & 1,0 & 0,828 & 1,0 & 1,0 & 0,869 & 1,0 & 1,0 \\
\hline \hline
\end{tabular}

Tabela B.3. Efeito do Parâmetro J nos Testes no Domínio do Tempo - Modelos AR(1)

\begin{tabular}{||l||ccc||ccc||}
\hline \hline \multicolumn{1}{||l||}{ Teste } & \multicolumn{2}{c||}{ Quenouille, $\mathrm{T}=100$} & \multicolumn{3}{c||}{ Mélard\&Roy, $\mathrm{T}=100$} \\
\hline \hline$\phi_{1}-\phi_{1} / \mathrm{J}$ & 2 & 4 & 7 & 3 & 5 & 8 \\
\hline \hline $0,2-0,2$ & 0,037 & 0,036 & 0,034 & 0,014 & 0,005 & 0,012 \\
0,3 & 0,069 & 0,053 & 0,034 & 0,031 & 0,014 & 0,006 \\
0,4 & 0,218 & 0,147 & 0,098 & 0,110 & 0,038 & 0,025 \\
0,5 & 0,473 & 0,347 & 0,235 & 0,283 & 0,132 & 0,048 \\
0,6 & 0,731 & 0,641 & 0,522 & 0,520 & 0,322 & 0,134 \\
0,7 & 0,941 & 0,863 & 0,809 & 0,822 & 0,553 & 0,270 \\
0,8 & 0,984 & 0,974 & 0,947 & 0,951 & 0,775 & 0,438 \\
\hline \hline
\end{tabular}


Tabela B.4. Efeito do Parâmetro J nos Testes no Domínio do Tempo - Modelos MA(1)

\begin{tabular}{||l||ccc||ccc||}
\hline \hline \multicolumn{1}{||l||}{ Teste } & \multicolumn{2}{|c||}{ Quenouille, $\mathrm{T}=100$} & \multicolumn{3}{c||}{ Mélard\&Roy, $\mathrm{T}=100$} \\
\hline \hline$\theta_{1}-\theta_{1} / \mathrm{J}$ & 2 & 4 & 7 & 3 & 5 & 8 \\
\hline \hline $0,2-0,2$ & 0,047 & 0,004 & 0,026 & 0,019 & 0,012 & 0,004 \\
0,3 & 0,053 & 0,004 & 0,026 & 0,026 & 0,011 & 0,002 \\
0,4 & 0,157 & 0,119 & 0,084 & 0,099 & 0,036 & 0,018 \\
0,5 & 0,344 & 0,246 & 0,180 & 0,214 & 0,108 & 0,045 \\
0,6 & 0,556 & 0,452 & 0,352 & 0,384 & 0,225 & 0,110 \\
0,7 & 0,734 & 0,676 & 0,528 & 0,551 & 0,423 & 0,176 \\
0,8 & 0,820 & 0,808 & 0,712 & 0,667 & 0,560 & 0,329 \\
\hline \hline
\end{tabular}




\section{Apêndice C}

\section{Teste de Kolmogorov-Smirnov}

Definição C.1 Seja $X_{1}, X_{2}, \ldots, X_{n}$ uma amostra aleatória de tamanho $n d a$ população com função de distribuição $F_{X}(x)$ cujas estatísticas de ordem são denotadas por $X_{(1)}, X_{(2)}, \ldots, X_{(n)}$. A função $F_{n}(x)$ dada por

$$
F_{n}(x)=\left\{\begin{array}{ccc}
0 & \text { se } & x<X_{(1)} \\
\frac{k}{n} & \text { se } & X_{(k)} \leq x<X_{(k+1)} \\
1 & \text { se } & x \geq X_{(n)}
\end{array}\right.
$$

denomina-se a função de distribuição empírica de $X$. Assim, $F_{n}(x)$ é a proporção de variáveis $X_{i}, \quad i=1,2, \ldots, n$ que não excedem o número $x$.

O lema de Glivenko-Cantelli estabelece que

$$
\sup _{-\infty<x<\infty}\left|F_{n}(x)-F_{X}(x)\right| \stackrel{\mathrm{P}}{\longrightarrow} 0
$$

indicando que a função de distribuição empírica de $X, F_{n}(x)$, é um estimador consistente para a função de distribuição de $X, F_{X}(x)$.

Se $X_{1}, X_{2}, \ldots, X_{n}$ é uma amostra aleatória de tamanho $n$ da população contínua $F_{X}(x)$, um problema de interesse é testar a hipótese

$$
H_{0}: F_{X}(x)=U_{X}(x) \text { para todo } x
$$

onde $U_{X}(x)$ é alguma função de distribuição contínua especificada.

Por outro lado, se considerarmos uma amostra aleatória de tamanho $n, X_{1}$, $X_{2}, \ldots, X_{n}$, da população contínua $F_{X}(x)$ e $Y_{1}, Y_{2}, \ldots, Y_{m}$ uma outra amostra de 
tamanho $m$, da população contínua $G_{Y}(x) \operatorname{com} G_{Y}(x)=P(Y<x)$ e onde todas as $m+n$ variáveis são mutuamente independentes, então um outro problema de interesse seria testar a hipótese

$$
H_{0}^{*}: F_{X}(x)=G_{Y}(x), \text { para todo } x .
$$

Kolmogorov e Smirnov propõem testes para as hipóteses $H_{0}$ dada por (C.1) e $H_{0}^{*}$ dada por (C.2), baseados nas funções de distribuição empírica de $X$ e de $Y$. Para a hipótese $H_{0}$ dada por (C.1), Kolmogorov(1933) sugere um teste baseado na estatística

$$
K_{n}=n^{1 / 2} \sup _{-\infty<x<\infty}\left|F_{n}(x)-F_{X}(x)\right| .
$$

O teste consiste em rejeitar $H_{0}$ dada por $(C .1)$ se $K_{n}$ é suficientemente grande. A distribuição de $K_{n}$ é independente de $F_{X}(x)$ se $H_{0}$ é verdadeira. Denotando esta distribuição por $\Phi_{n}(x)$, Kolmogorov(1933) prova que

$$
\lim _{n \rightarrow \infty} \Phi_{n}(x)=\lim _{n \rightarrow \infty} P\left(K_{n}<x\right)=\Phi(x)=\sum_{j=-\infty}^{\infty}(-1)^{j} e^{-2 j^{2} x^{2}} \quad 0<x<\infty .
$$

Smirnov(1939) sugere um teste similar para a hipótese $H_{0}^{*}$ dada por (C.2), baseado na estatística

$$
D_{m, n}=\left(\frac{m n}{m+n}\right)^{1 / 2} \sup _{-\infty<x<\infty}\left|F_{n}(x)-G_{m}(x)\right|
$$

com $G_{m}(x)$ a função de distribuição empírica da amostra $Y_{1}, \ldots, Y_{m}$, definida de forma similar a $F_{n}(x)$.

Rejeita-se $H_{0}^{*}$ se $D_{m, n}$ é suficientemente grande. Denotando a distribuição de $D_{m, n}$ por $\Phi_{m, n}, \operatorname{Smirnov}(1939)$ prova que se $0<a \leq \frac{m}{n} \leq b<\infty$, quando $m \rightarrow \infty$ e $n \rightarrow \infty$, então a distribuição limite de $D_{m, n}$ é a distribuição $\Phi$ dada por (C.3).

Para maiores detalhes consultar, Conover(1980), Darling(1956) e Gibbons(1985).

Para efeitos práticos, ambos os testes estão implementados no S-Plus. 


\section{Apêndice D}

\section{Programas no S-Plus}

Algoritmos Utilizados nos Testes Univariados.

Algoritmo Newton-Raphson (newrap) :

Estima os parâmetros $\alpha, \beta, \gamma$ de máxima verossimilhança do modelo logístico proposto em Coates e Diaggle(1986).

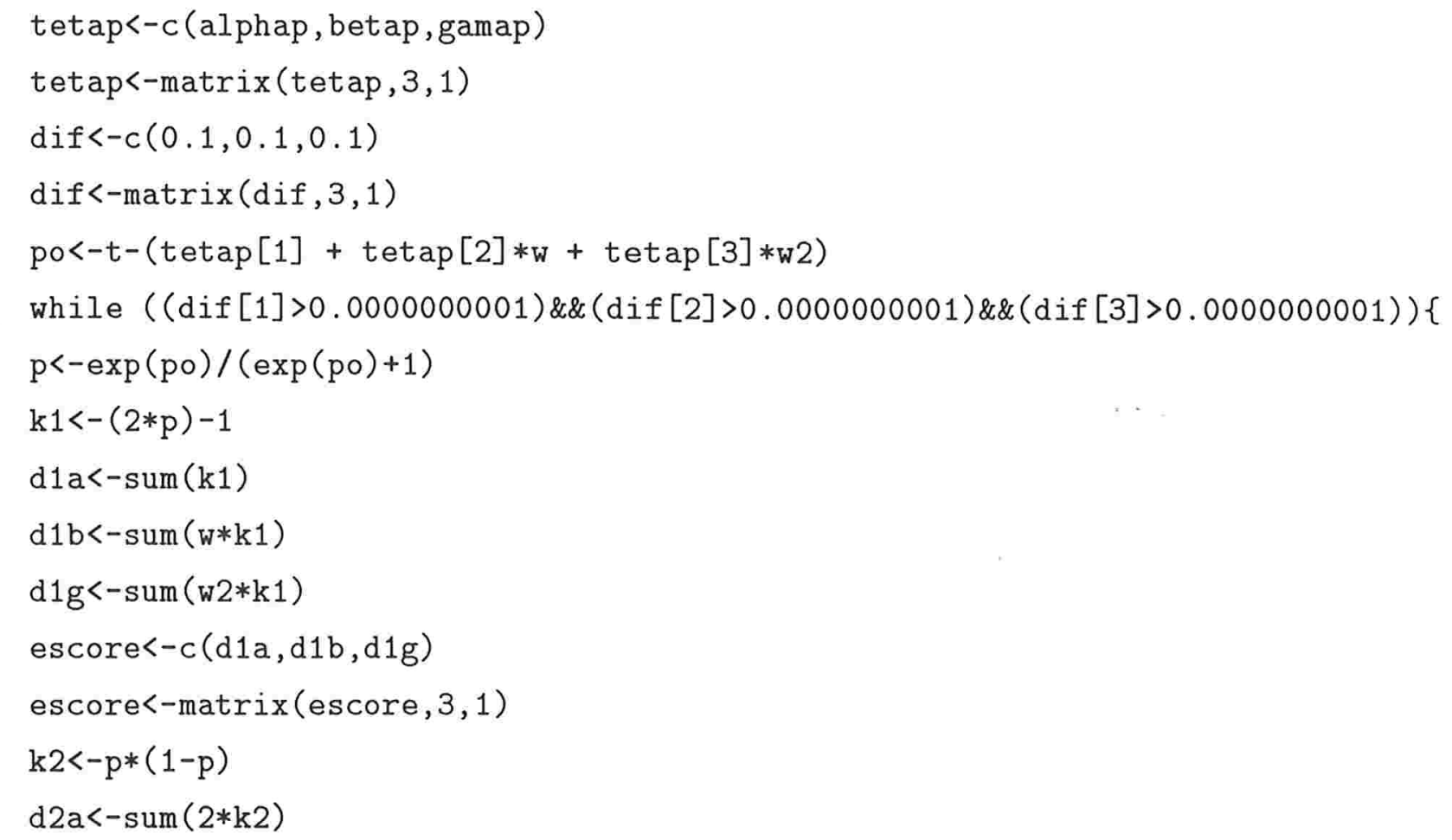




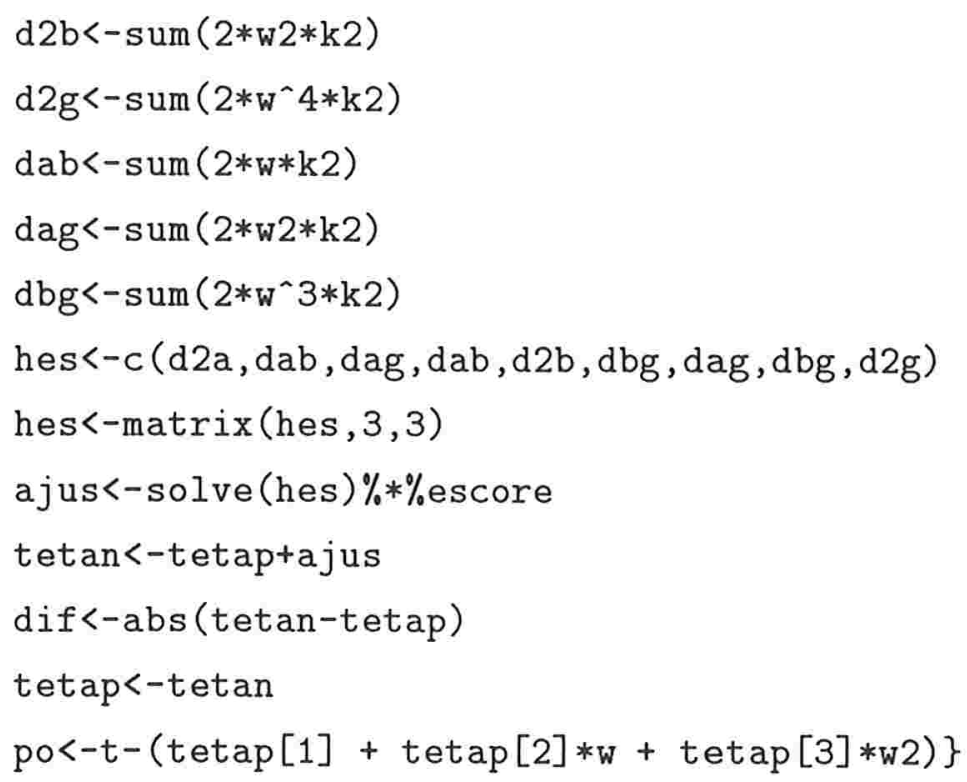

\section{Algoritmo razaoveros:}

Encontra a estatística da razão de verossimilhança e seu nível descritivo como em Coates e Diggle(1986). As duas séries de entrada são sx e sy, neste caso simuladas.

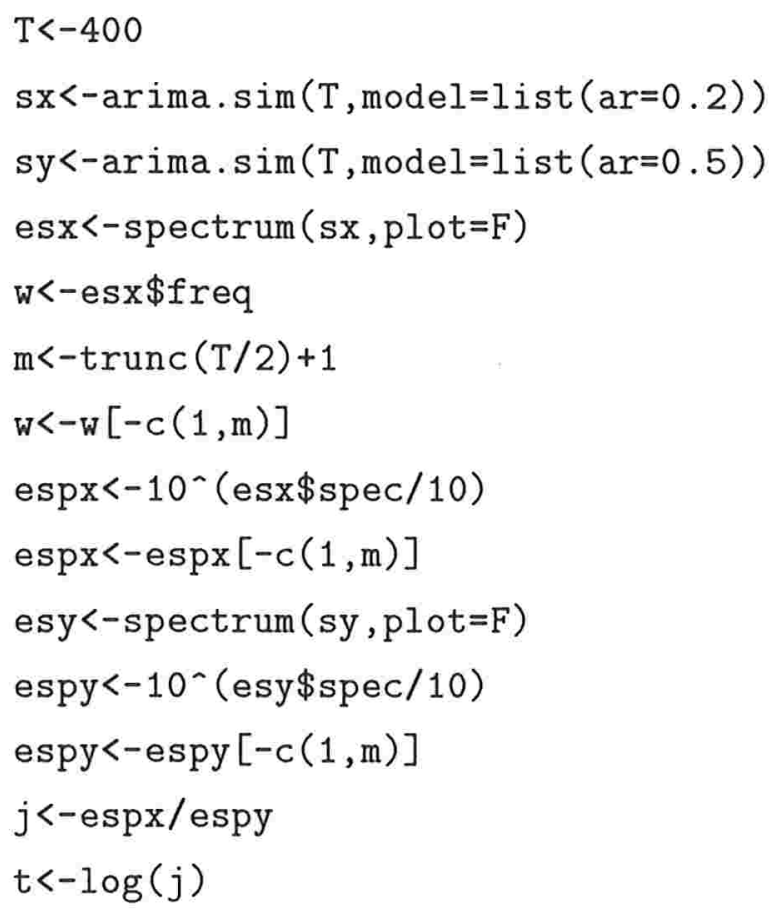




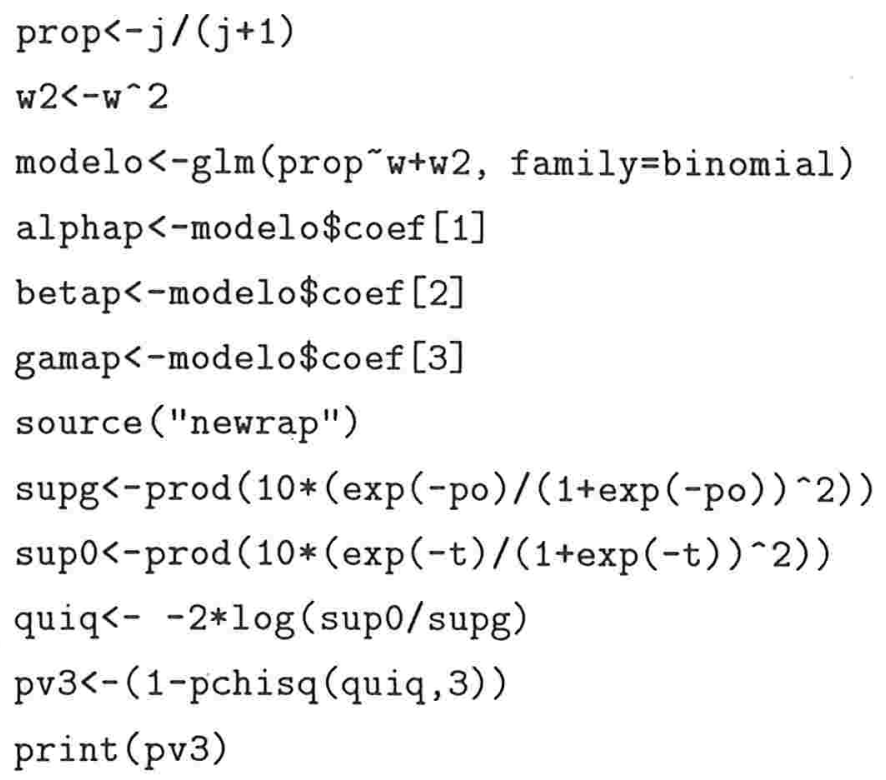

\section{Algoritmo somacum:}

Encontra a estatística de Kolmogorov-Smirnov e seu nível descritivo para o teste das somas acumuladas proposto em Coates e Diggle(1986). As duas séries de entrada são sx e sy, neste caso simuladas.

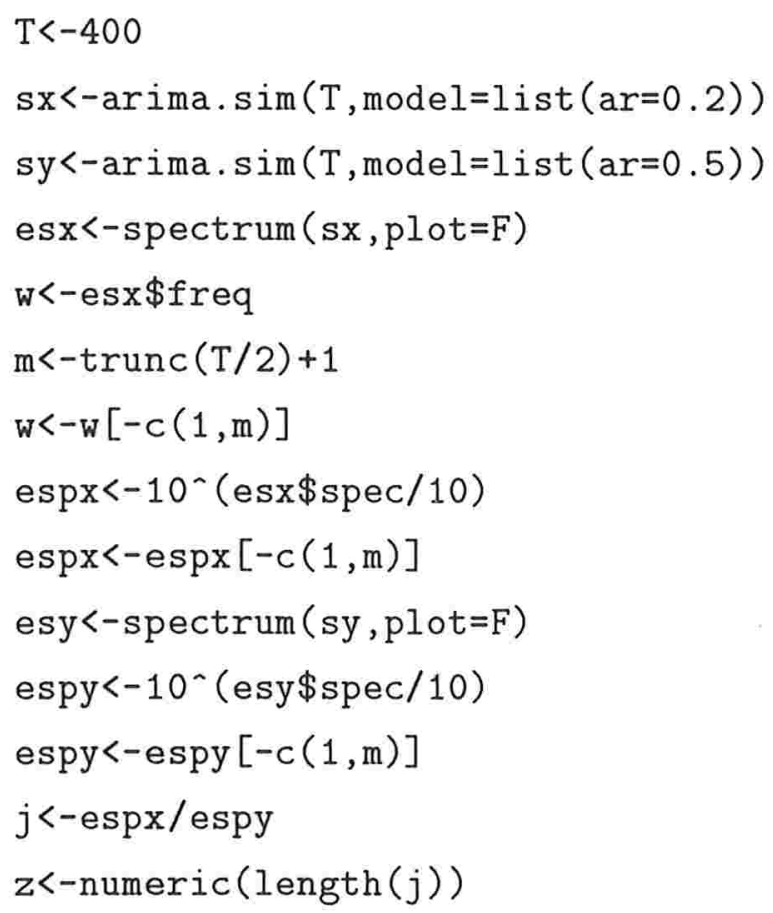




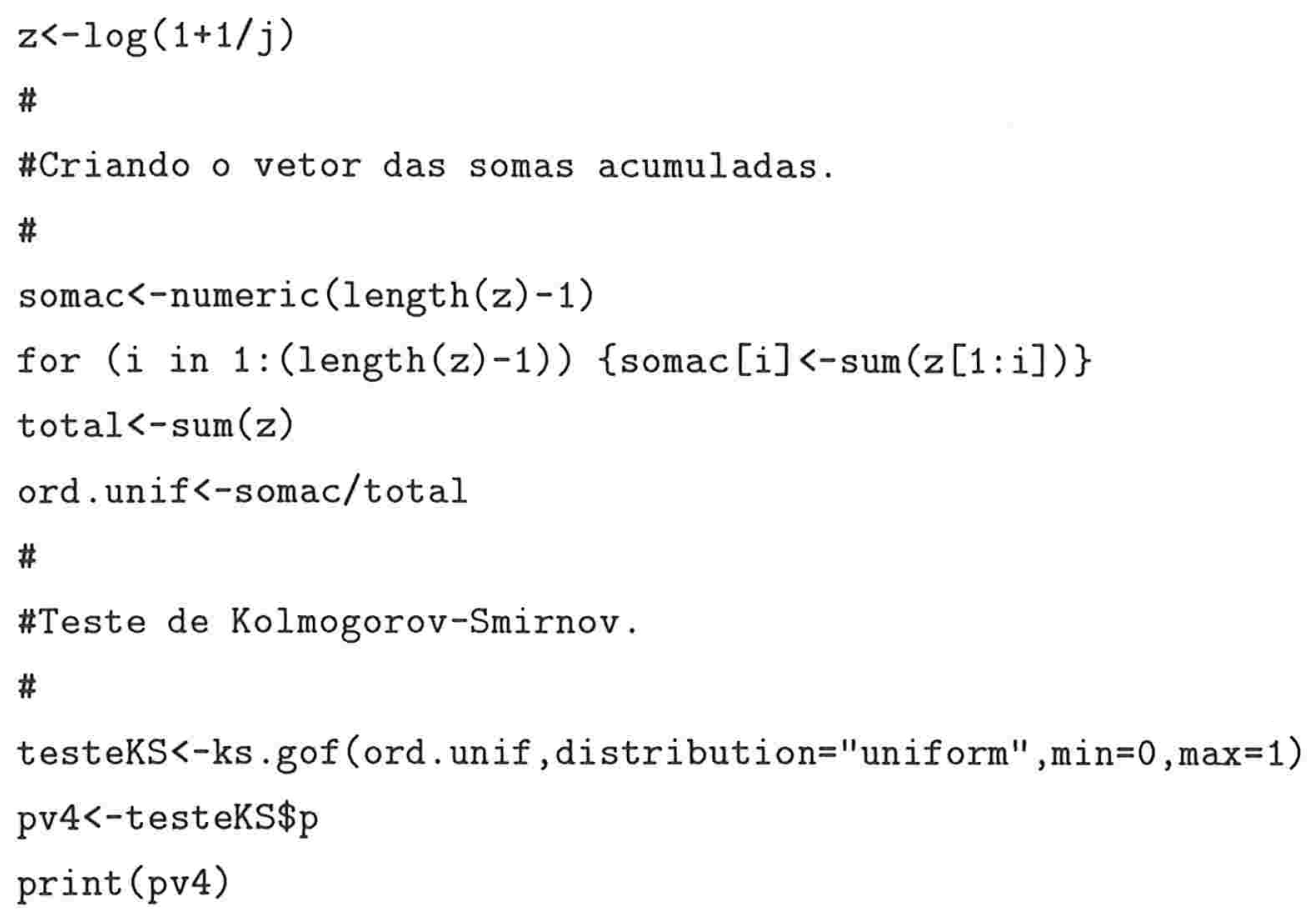

\section{Algoritmo fautcov:}

Calcula a matriz de covariâncias (proposta por Mélard e Roy (1984)) da função de autocovariâncias da série sx. Neste caso sx é simulada.

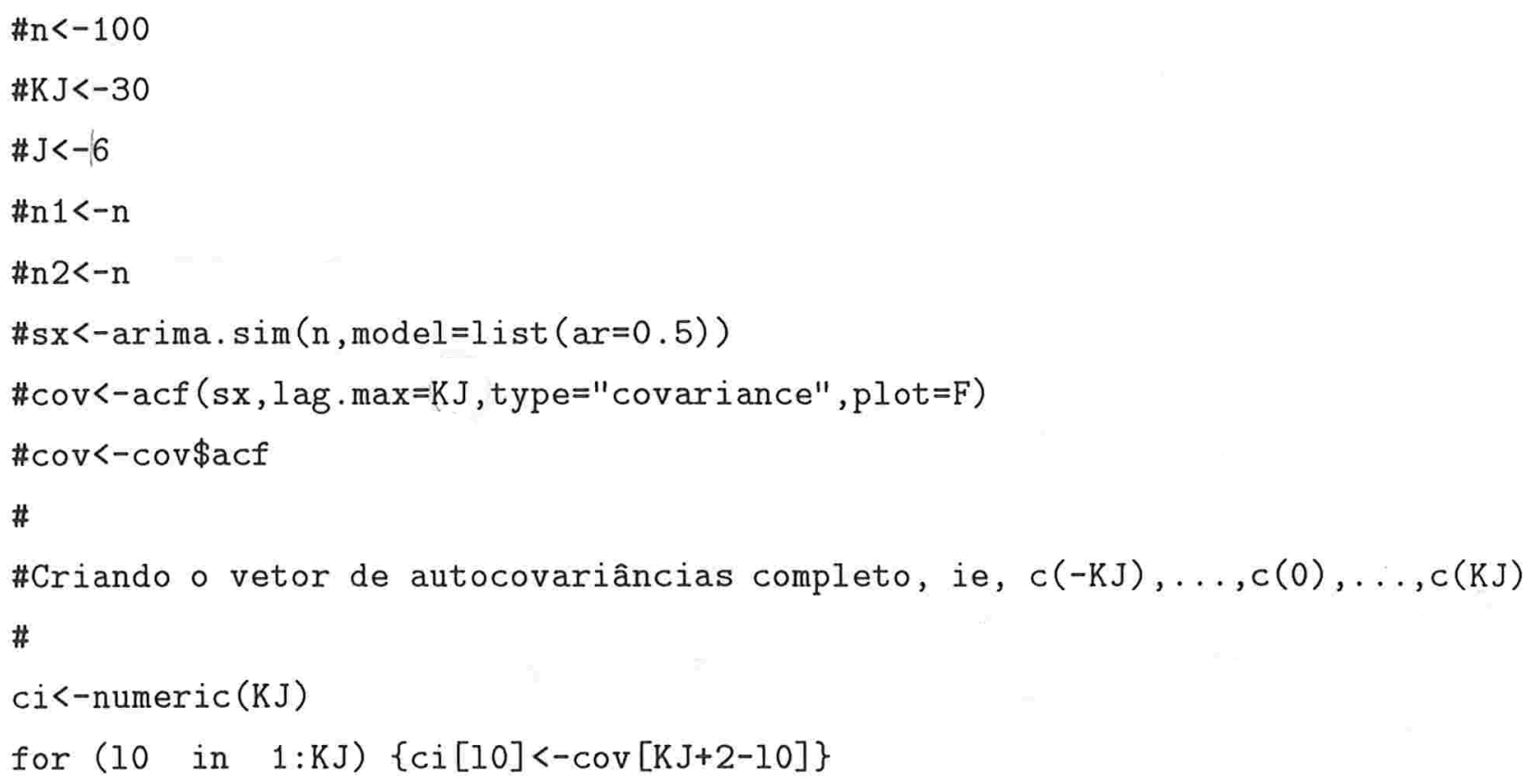




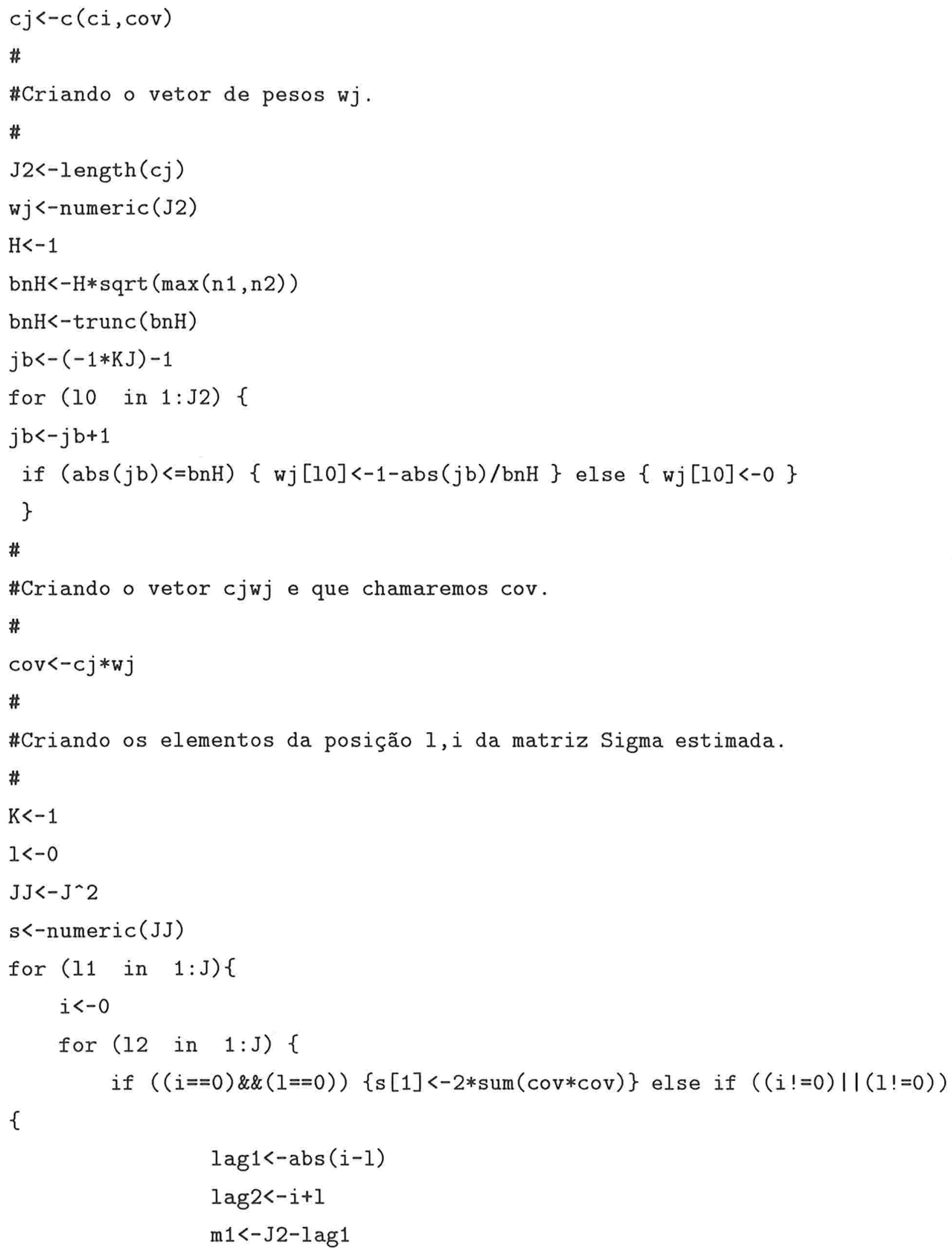




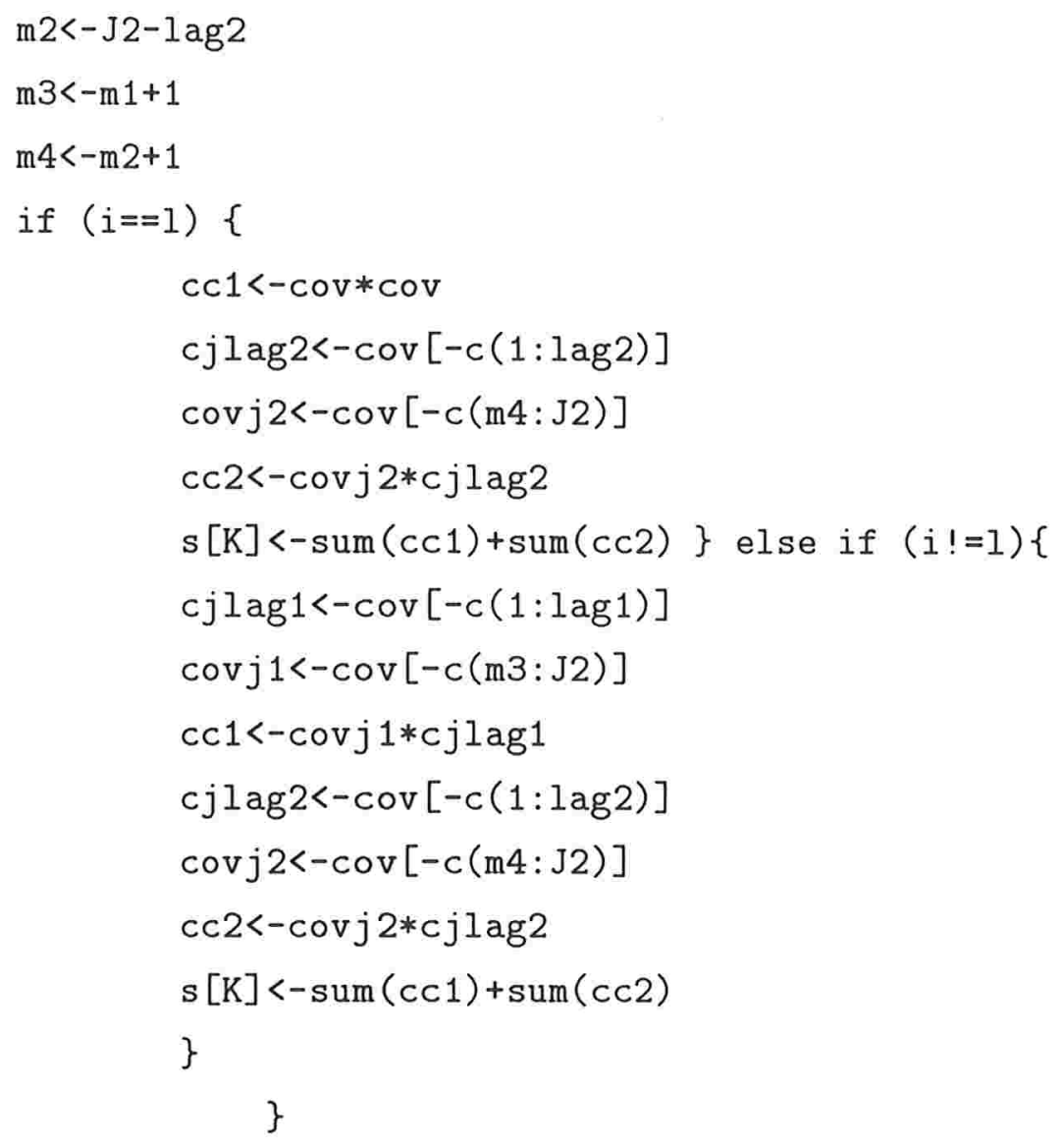

\section{Algoritmo Qestat:}

Calcula a estatística de Mélard e Roy(1984). J é o número de lag's ou autocovariâncias a serem comparadas incluindo o lag zero. 


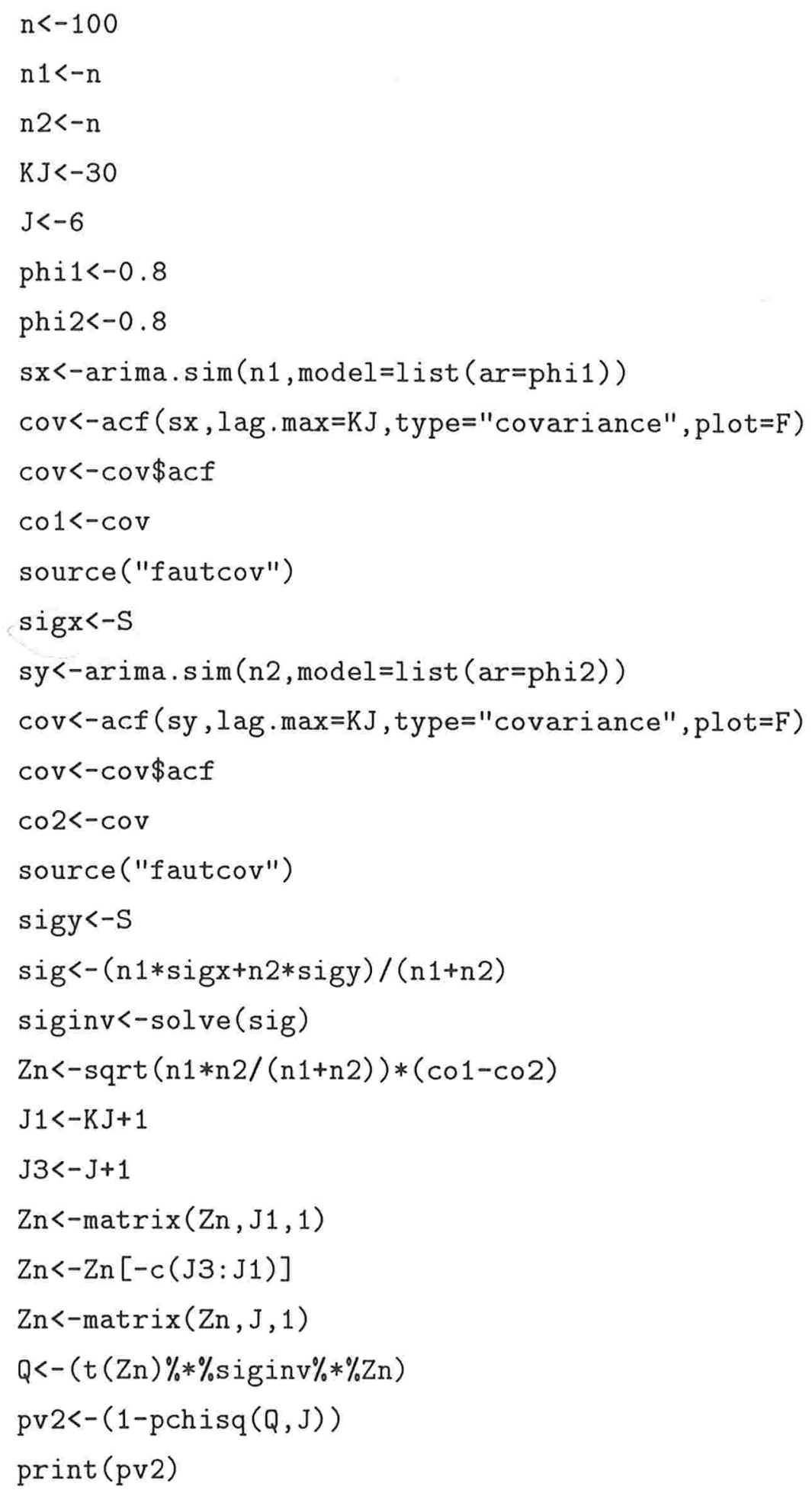




\section{Algoritmo facp:}

Calcula a função de autocorrelação parcial de uma série a partir da FAC, resolvendo as equações de Yule-Walker. vetmat é o vetor que contém todas as matrizes $\mathrm{R}$ de tamanho pxp segundo Box et al(1994). phi e o vetor que vai conter os valores da FACP.

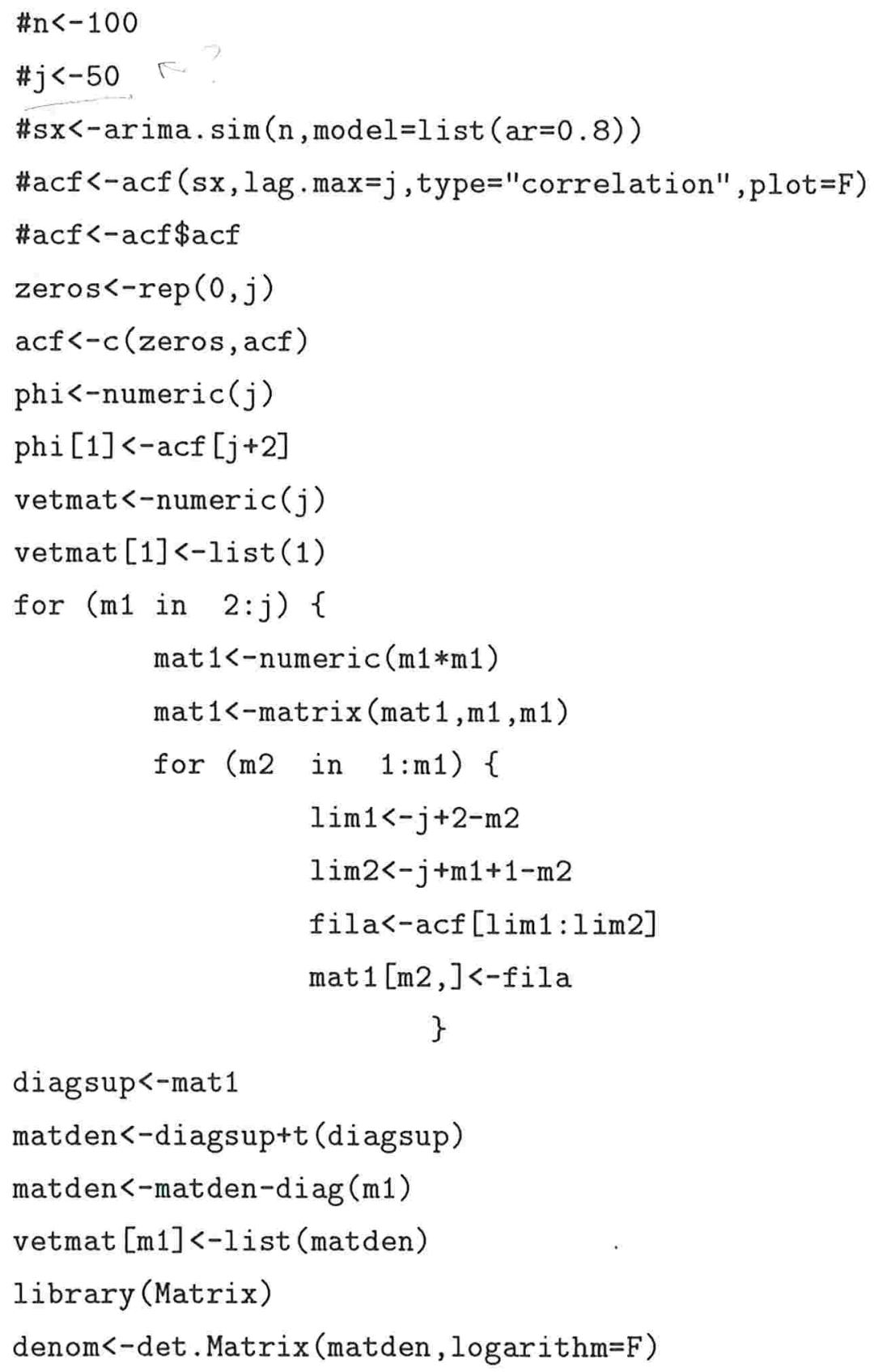




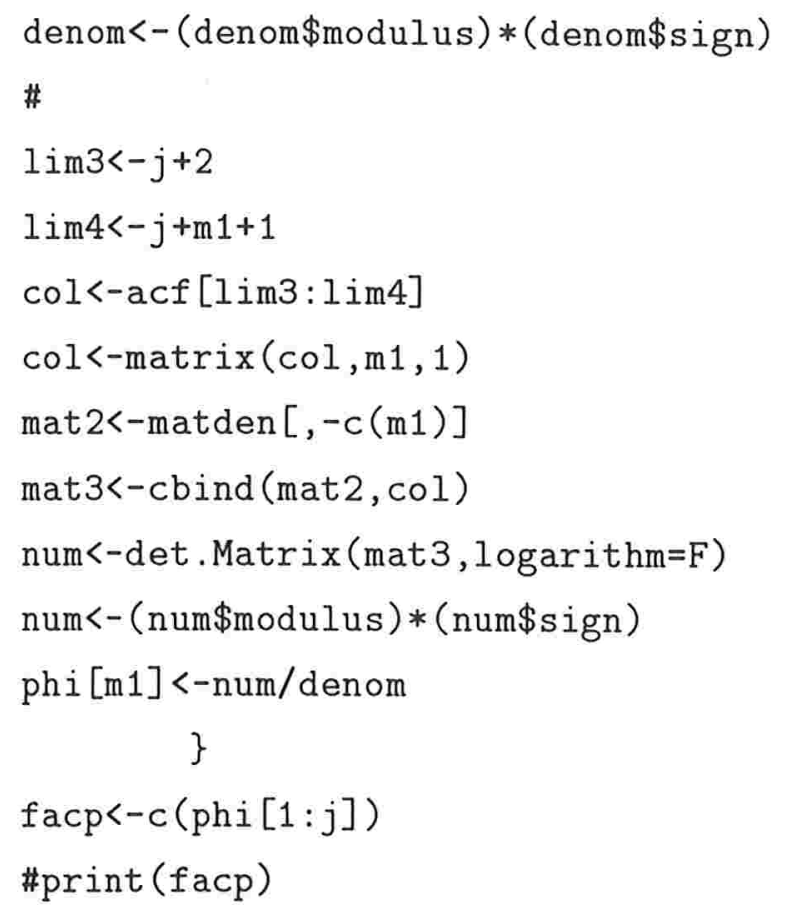

\section{Algoritmo quenou:}

Calcula a estatística e o nível descritivo para o teste proposto por Quenouille (1958) para comparar duas funções de autocorrelação. P é o número de funções de autocorrelação utilizadas no teste.

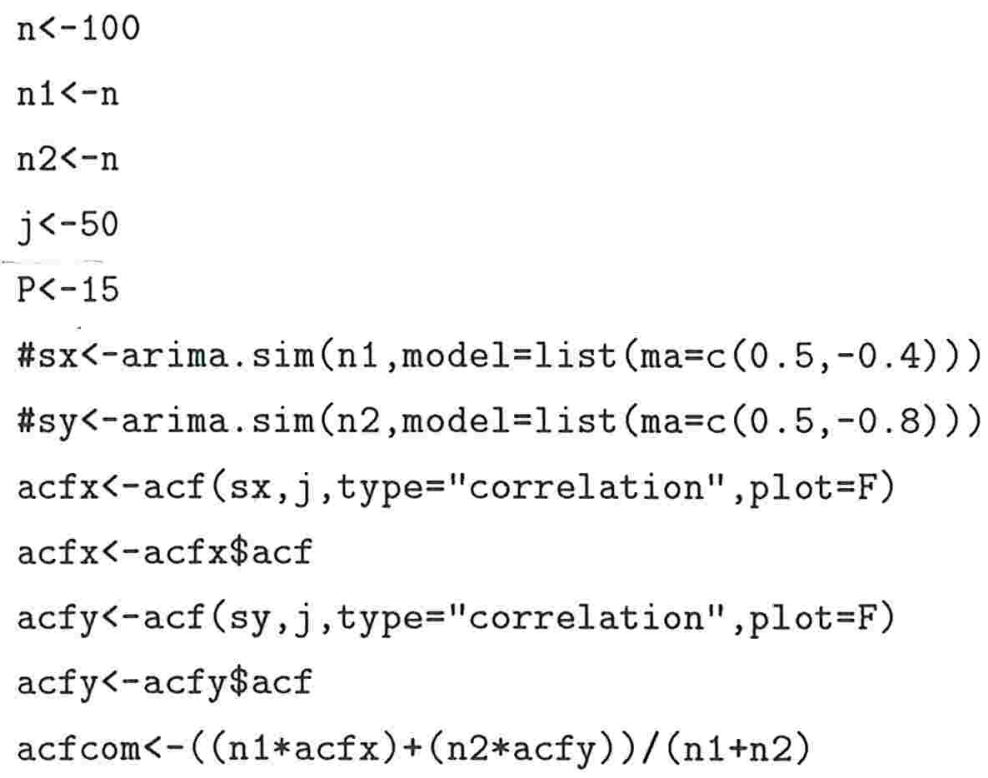




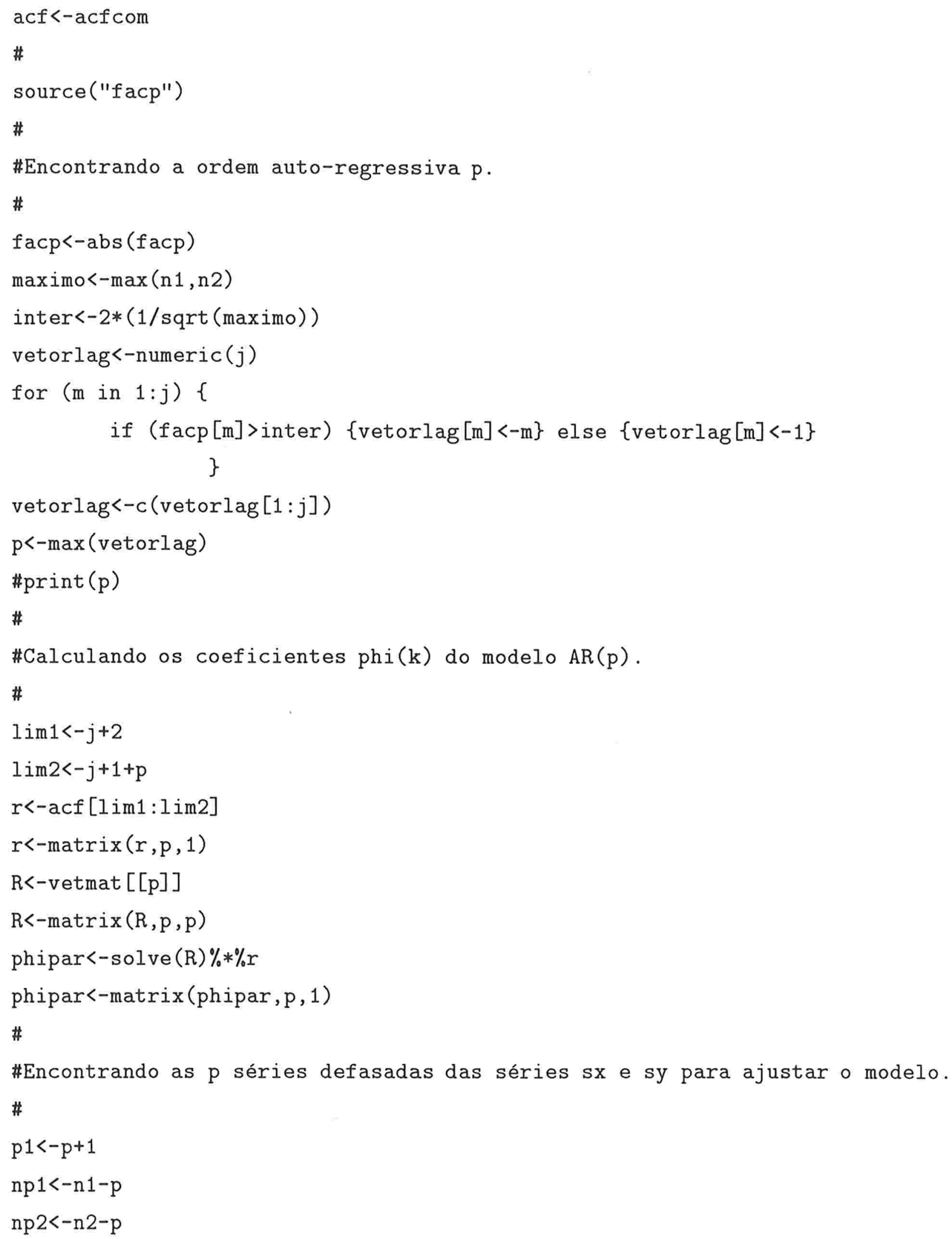




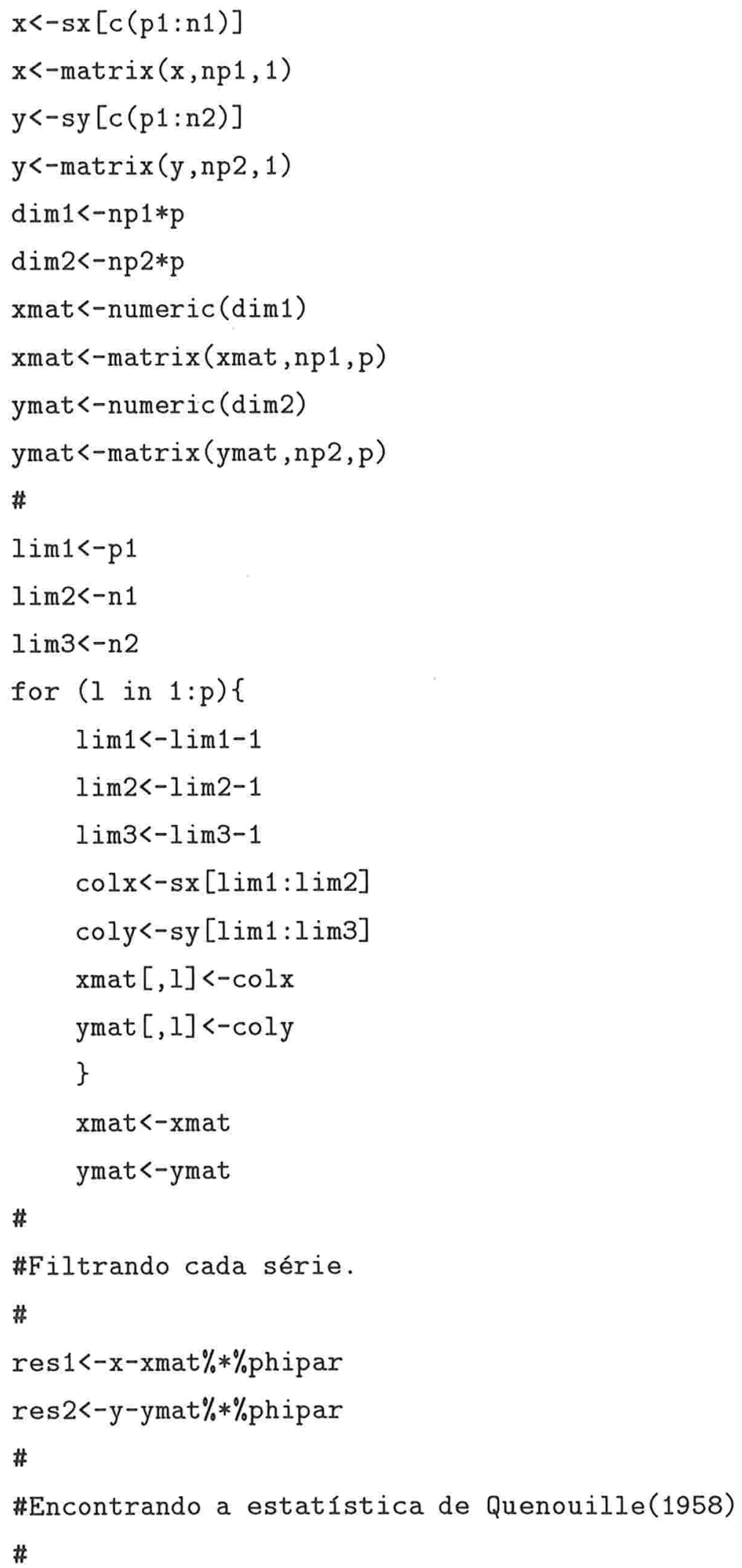




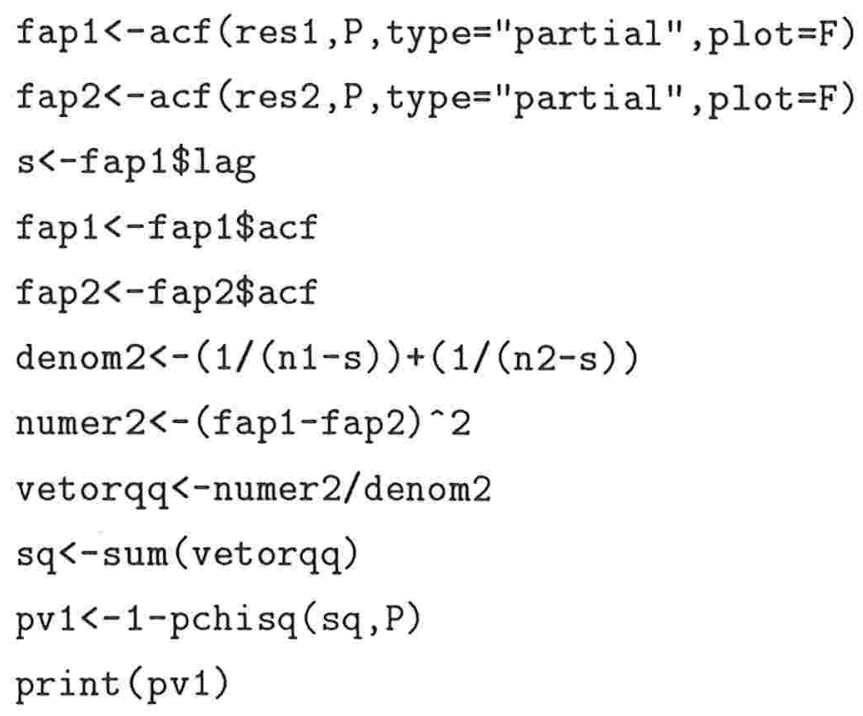




\section{Funções Utilizadas nos Testes Multivariados}

As funções seguintes são transcritas do original de Carmona e Wang (1996).

\section{Function est.spectral.matrix}

Compute the 4 by 4 spectral density matrix estimate for the given vectorvalued series

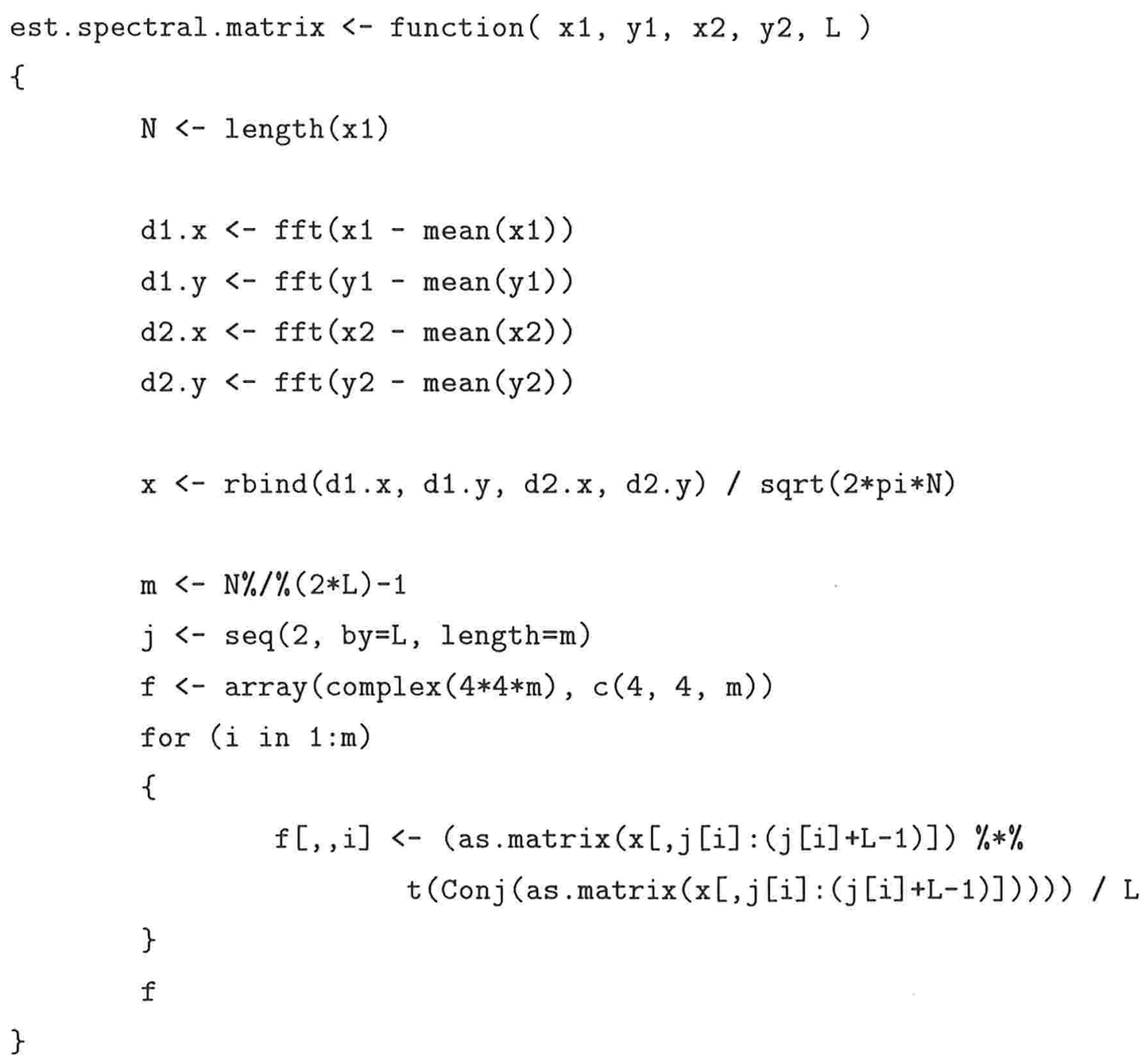


plot the modulus of the 4 by 4 spectral density matrix estimate at all Calculated frequencies with the same scale in each panel.

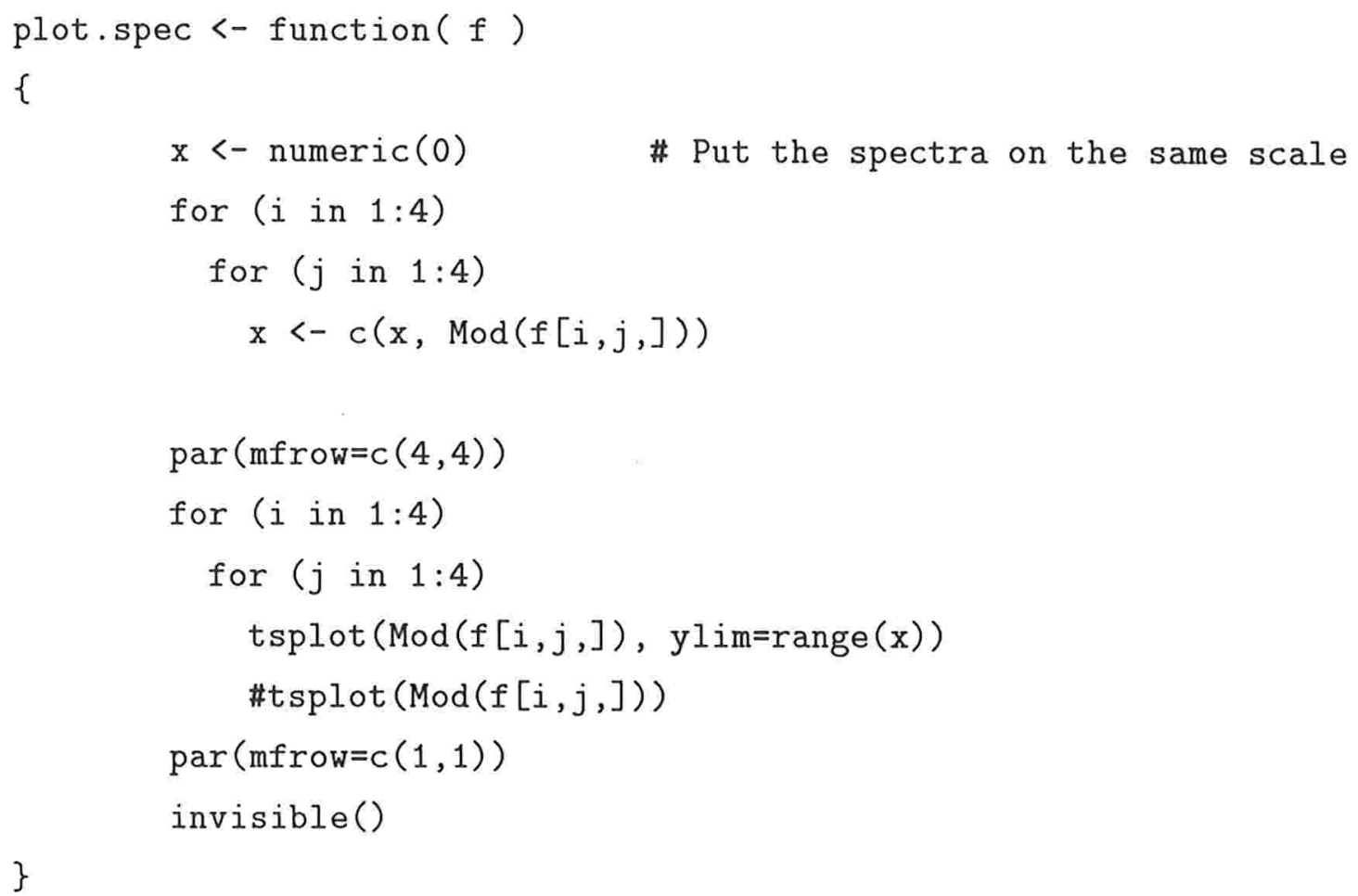

Function sqrt.matrix

Compute the (inverse) square root of a matrix

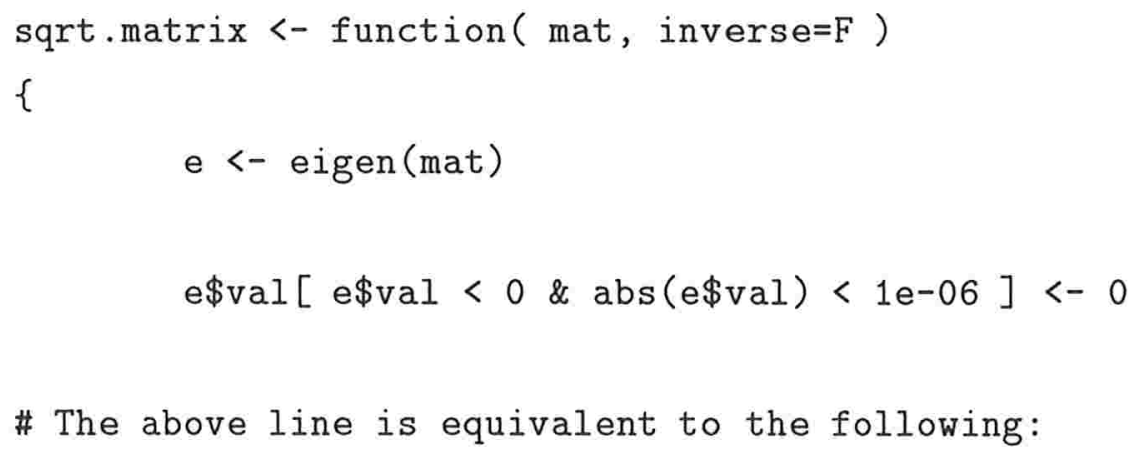




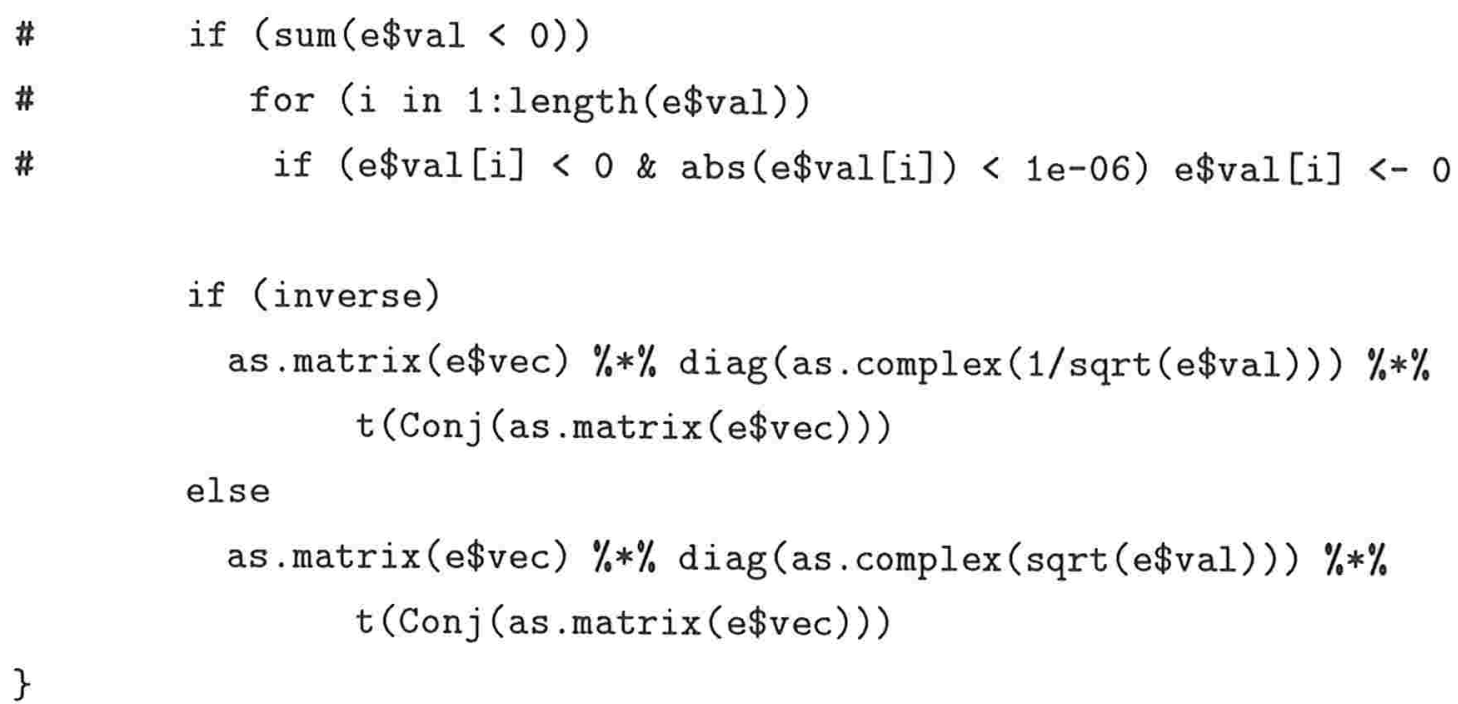

\section{Function compute.coh}

Compute the coherency from the spectral density estimate at all calculated frequencies.

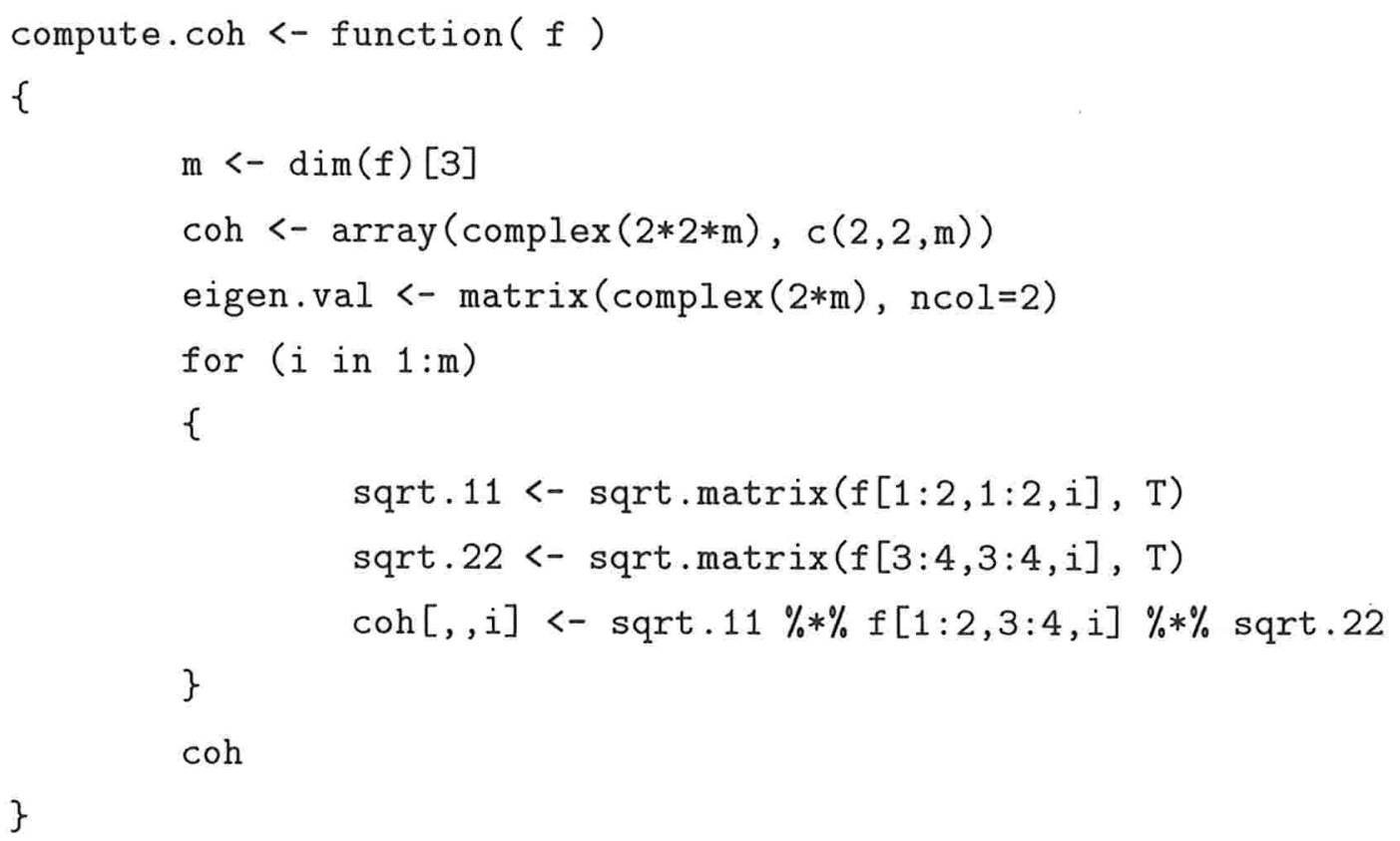

Function sim.dep 
Simulate $\mathrm{N}$ by 4 data with the given spectral density matrix (in some sense)

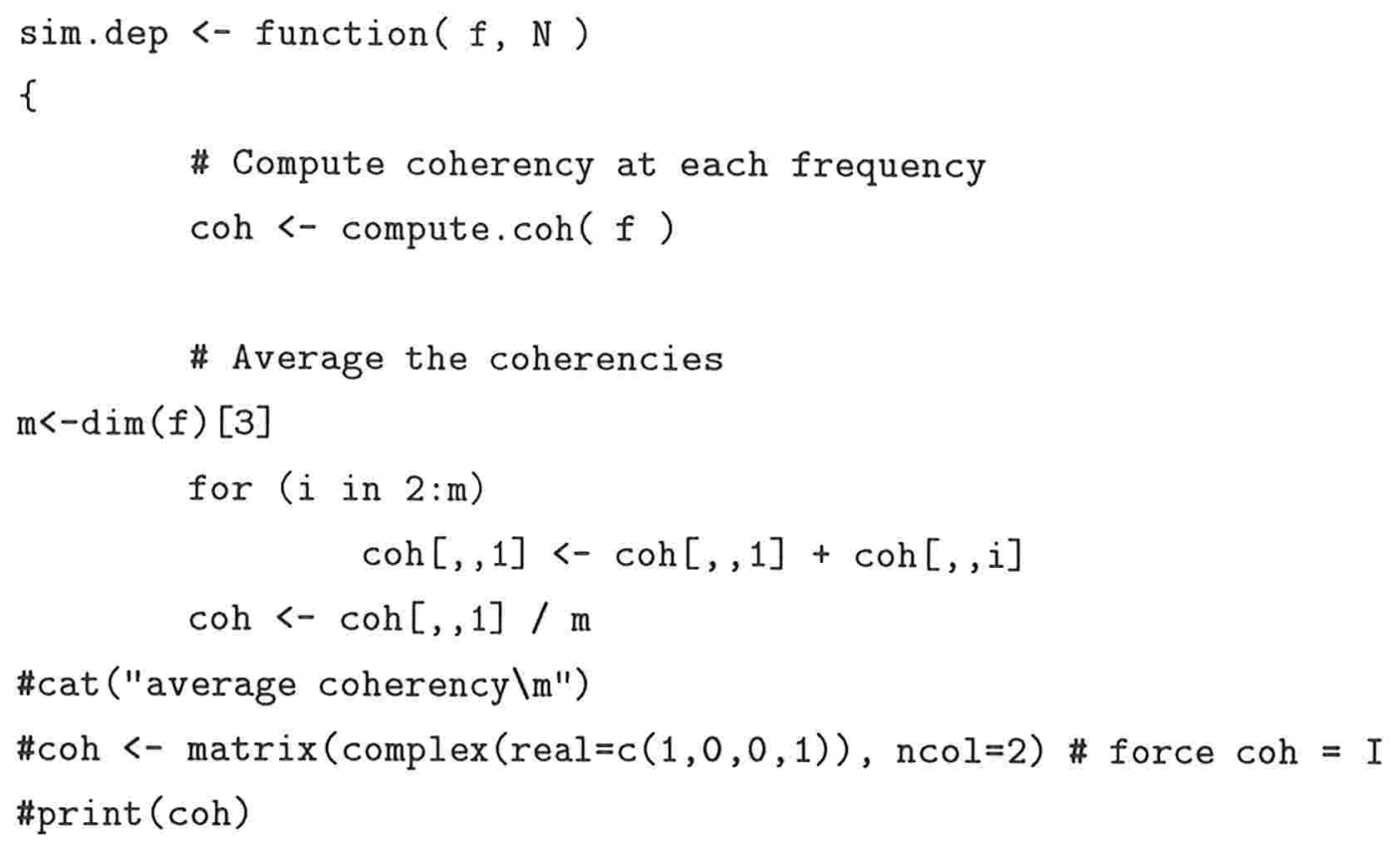




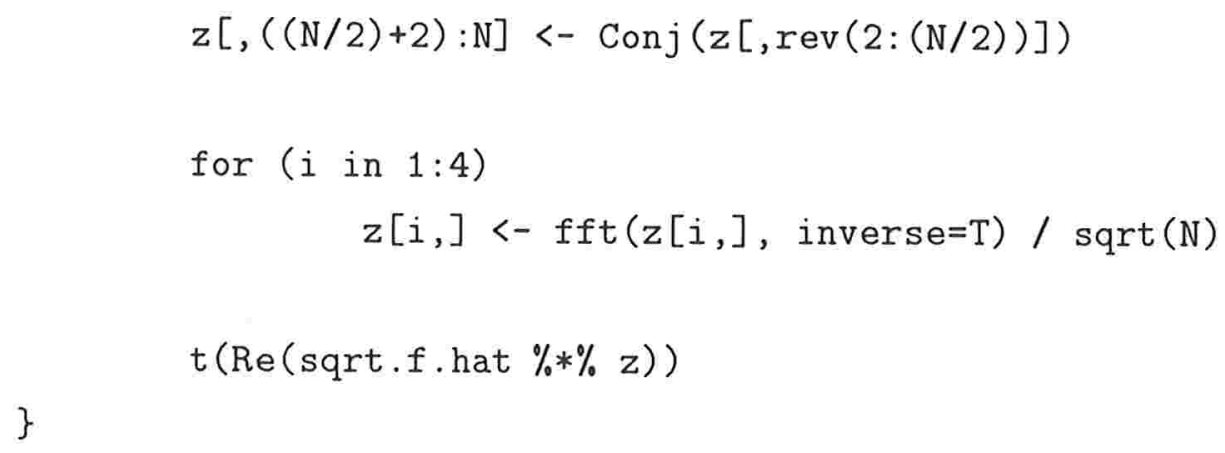

Function trace.stat

Compute the "trace" statistic of the spectral density matrix

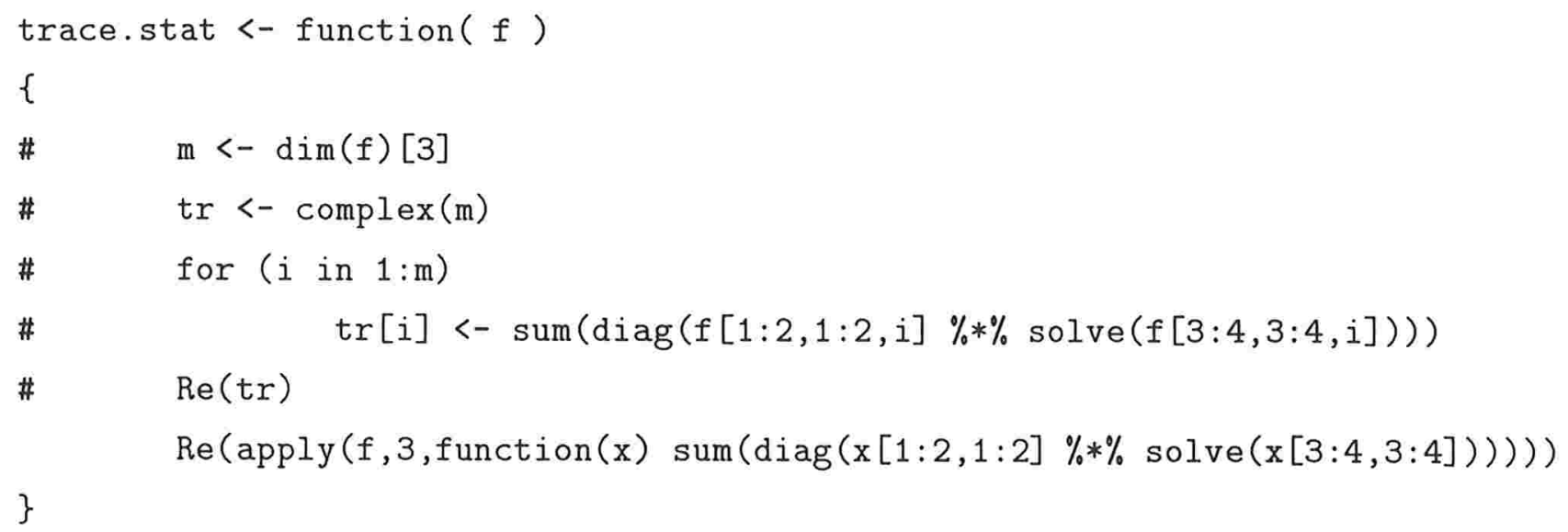

Function det.stat

Compute the "determinant" statistic of spectral density matrix

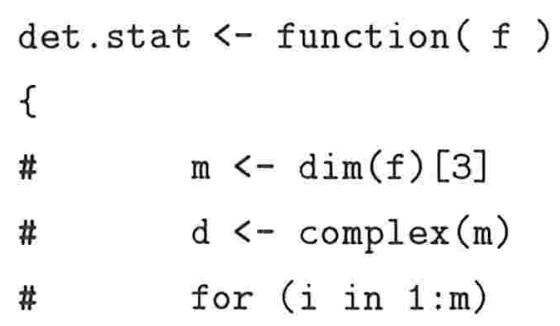




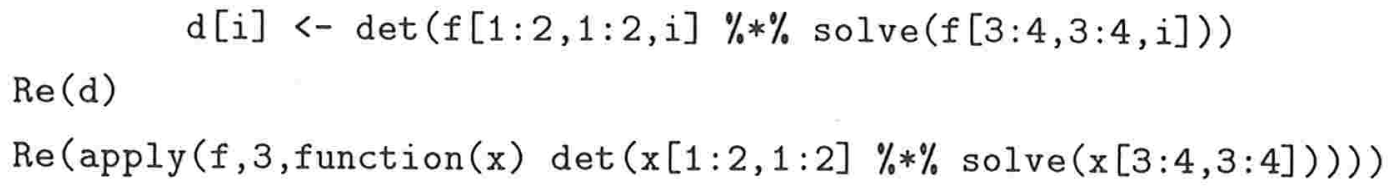

Function wishart.ratio

\section{ALGORITMO WISHART.S}

Compute the statistic trace $\left.\left(W 1 * W 2^{(}-1\right)\right)$ or $\left.\operatorname{det}\left(W 1 * W 2^{(}-1\right)\right)$

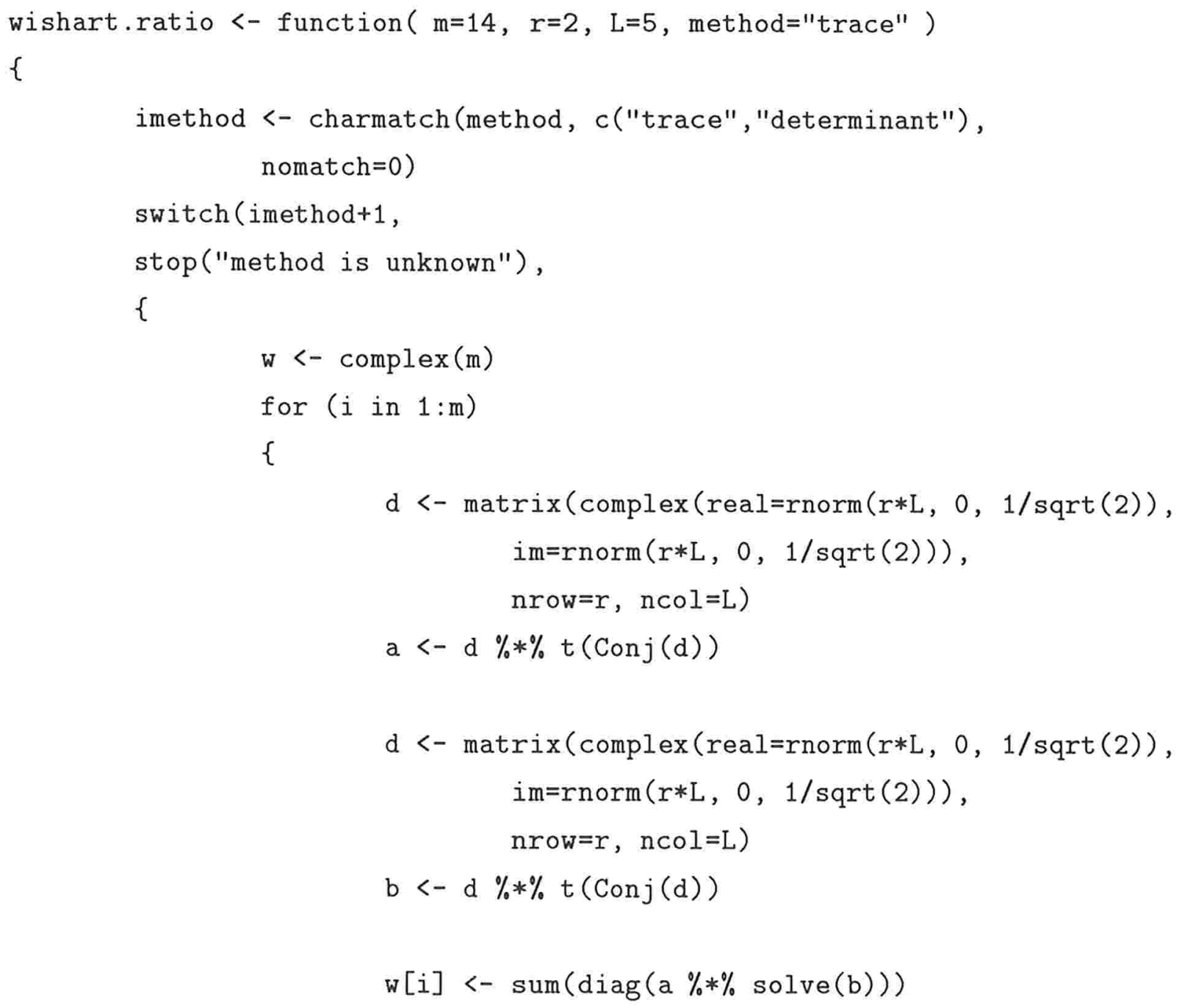




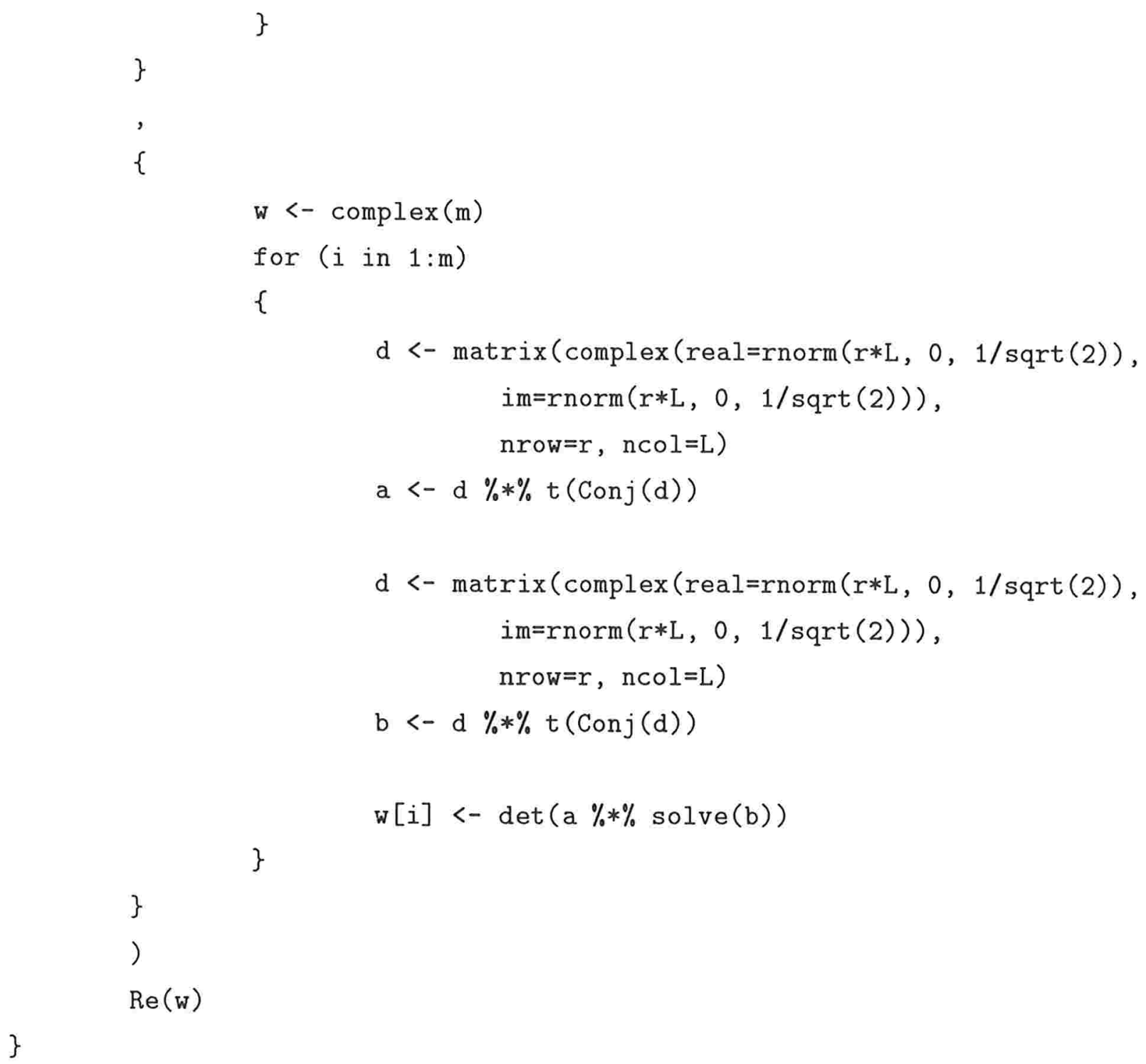

Function det

Compute the determinant of a square matrix The code is copied from p. 54 of Phil Spector's S book

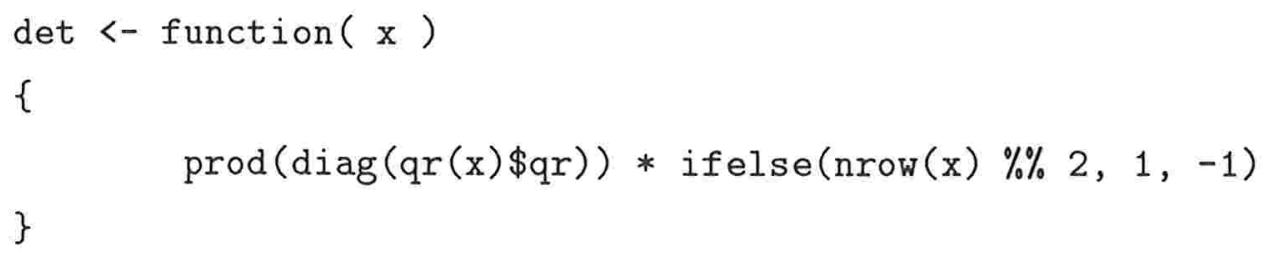




\section{Function kstwo}

Kolmogorov-Smirnov two-sample test.

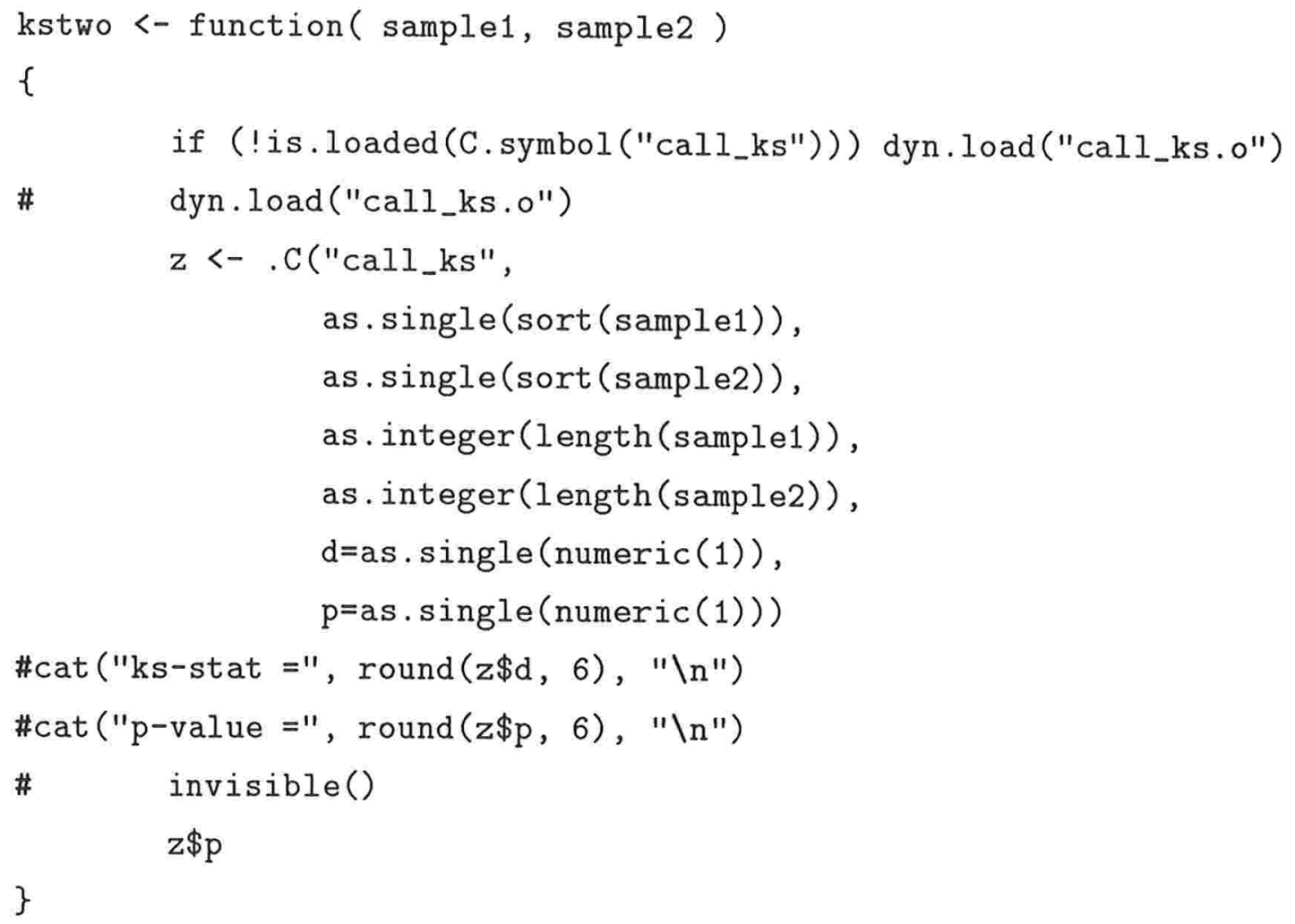

Function T.boot

Compute the B bootstrap statistics.

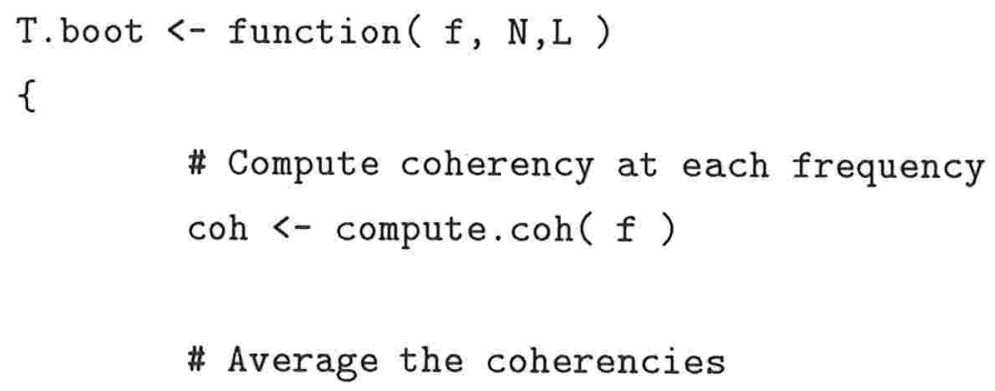




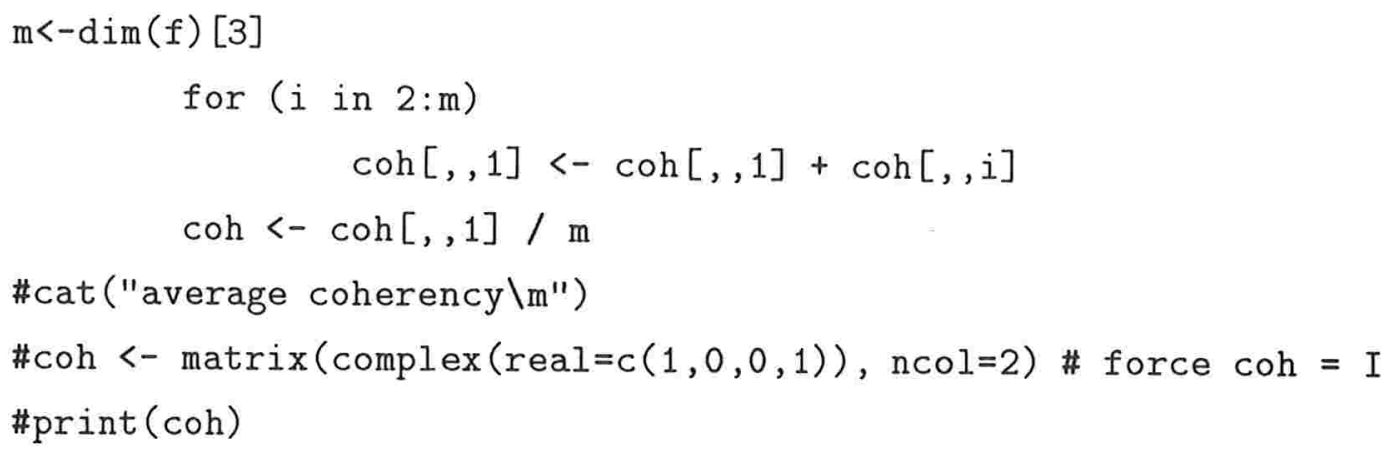




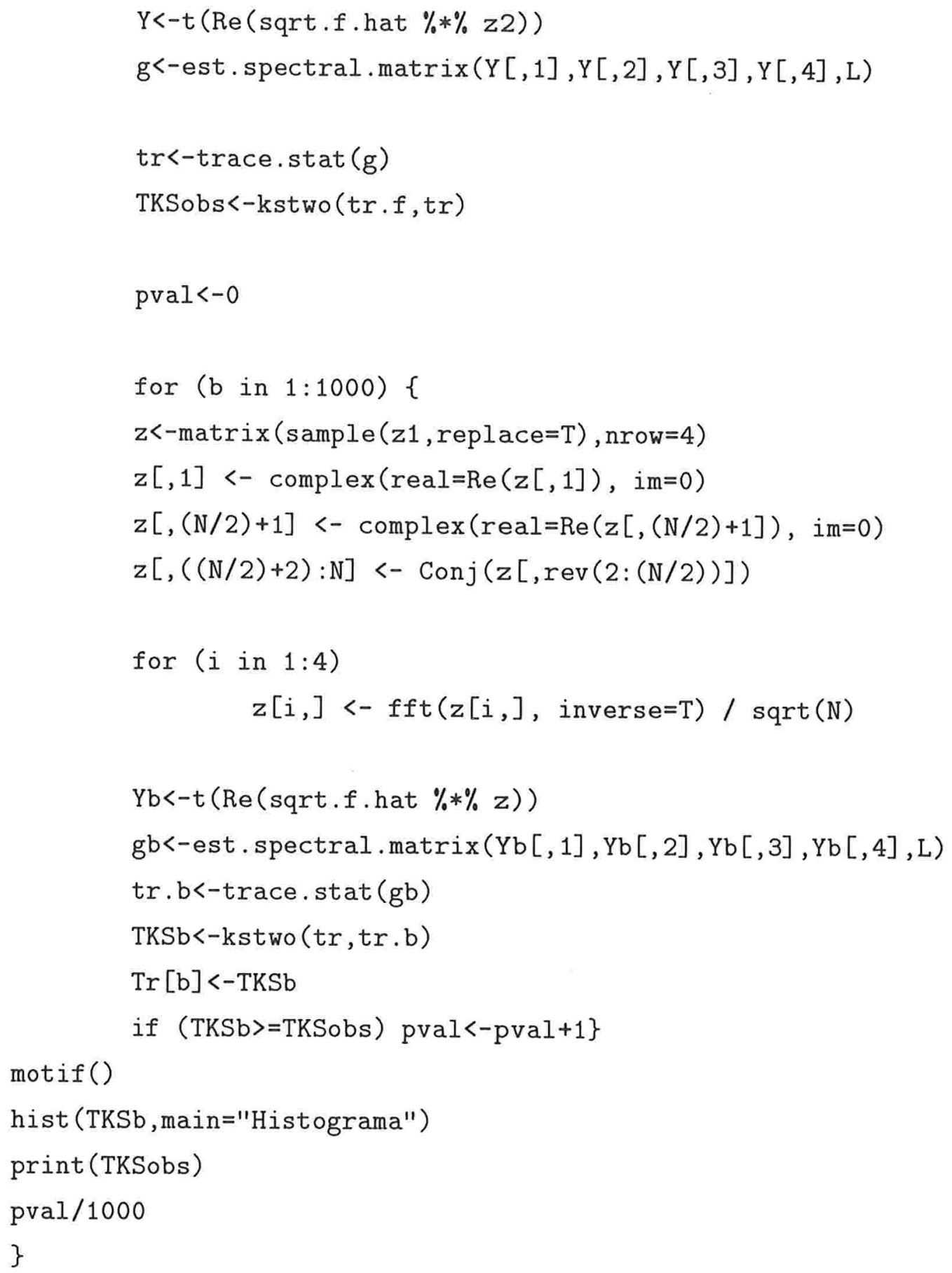




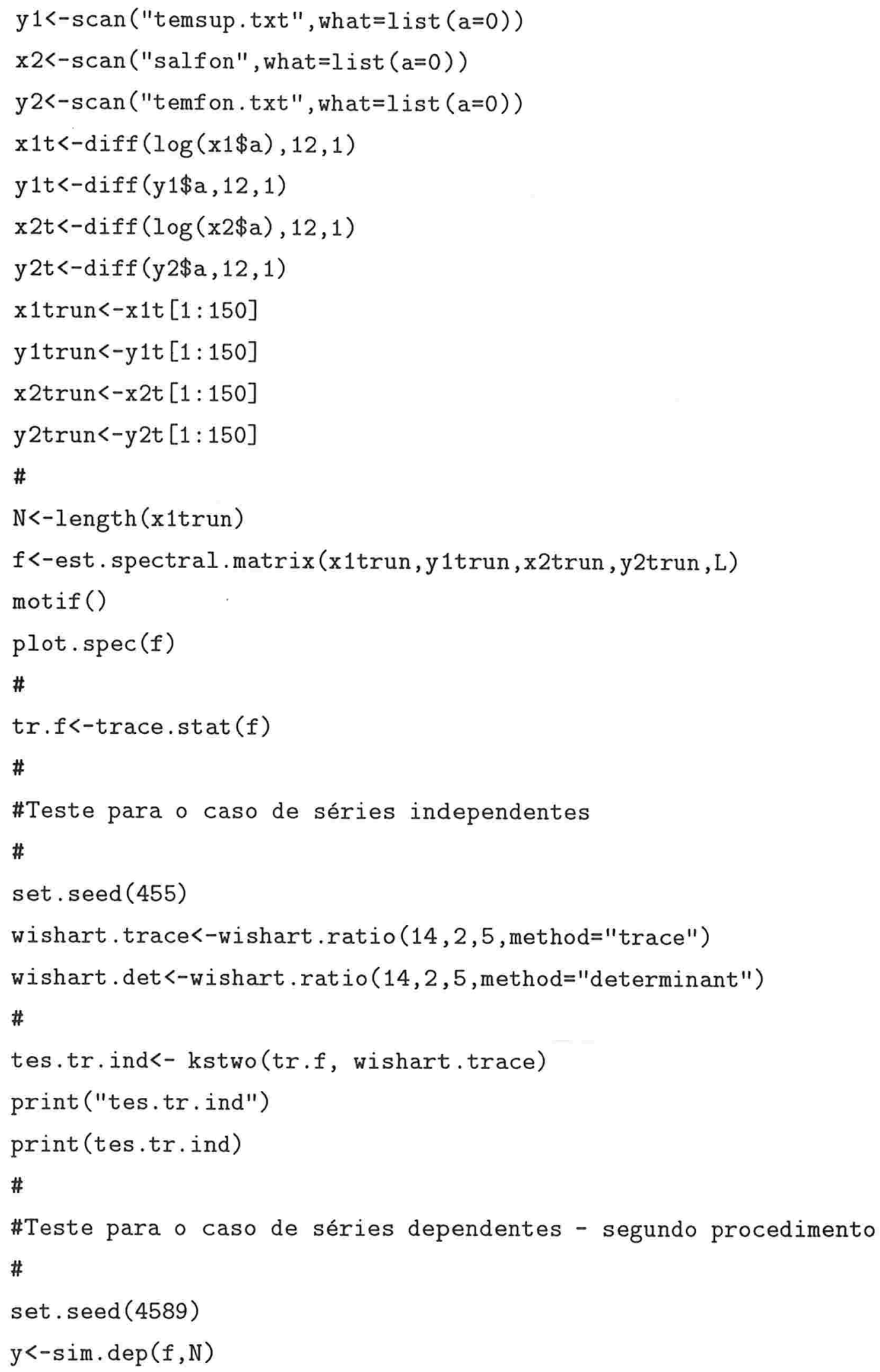




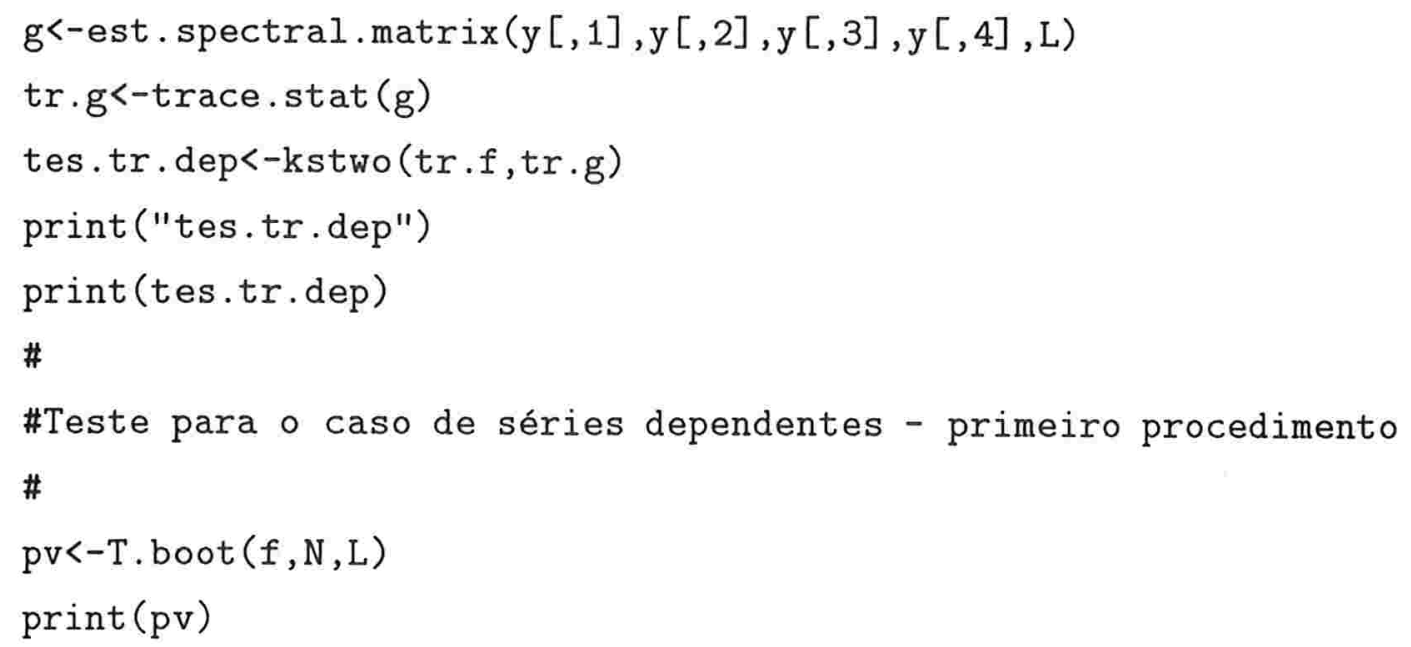

